Antennas for Light and Plasmons
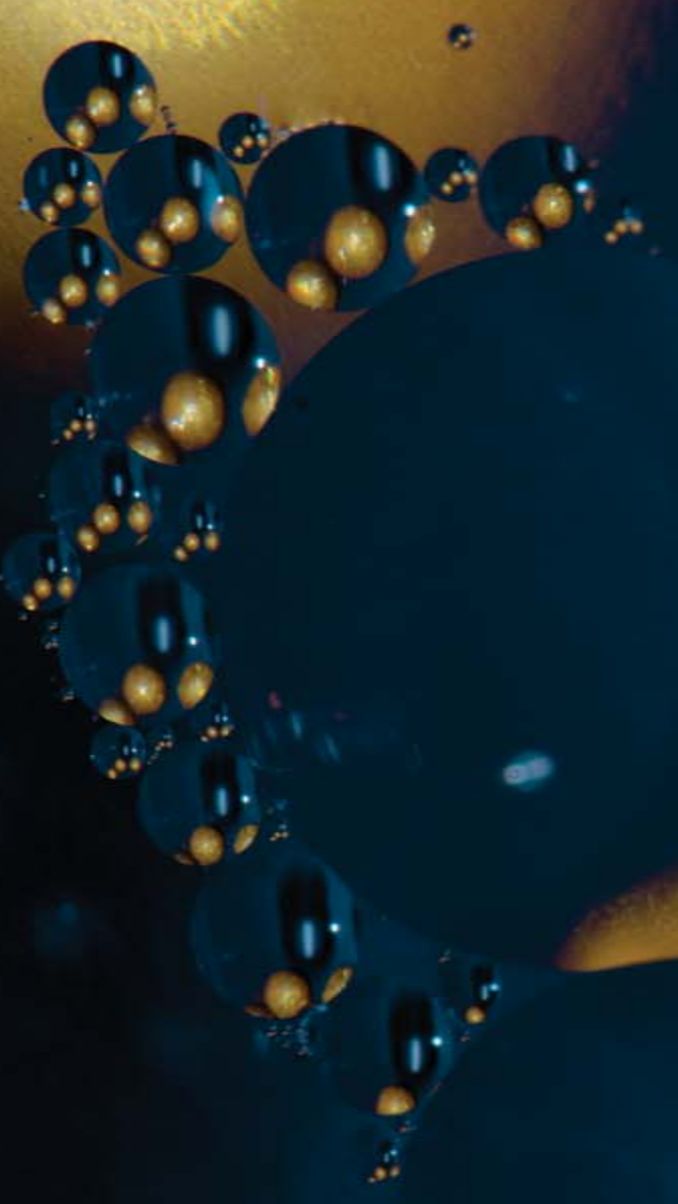

Dirk Jan Dikken 
Antennas for Light and Plasmons 
Samenstelling van de promotiecommissie:

Prof. dr. Jennifer L. Herek Universiteit Twente

Prof. dr. Klaus J. Boller Universiteit Twente

Prof. dr. Martin P. van Exter Universiteit Leiden

Prof. dr. Bert Hecht University of Würzburg

Prof. dr. Kobus Kuipers Universiteit Utrecht

Prof. dr. Allard P. Mosk Universiteit Twente

Dr. Jord C. Prangsma Ibsen Photonics

This work was carried out at the Optical Sciences group, which is part of:

Department of Science and Technology

and MESA+ Institute for Nanotechnology,

University of Twente, P.O. Box 217,

7500 AE Enschede, The Nederlands.

Financial support was provided by a VICI grant from the CW section of the Nederlandse Wetenschappelijke Organisatie (NWO) to Prof. Jennifer L.

Herek.

ISBN: 978-90-365-3953-1

DOI: $10.3990 / 1.9789036539531$

Author email: dirkjan.dikken@gmail.com

Copyright (C) 2015 by Dirk Jan Dikken

All rights reserved. No part of the material protected by this copyright notice may be reproduced or utilized in any form or by any means, electronic or mechanical, including photocopying, recording or by any information storage and retrieval system, without the prior permission of the author. 


\section{Antennas For Light And Plasmons}

\section{PROEFSCHRIFT}

ter verkrijging van

de graad van doctor aan de Universiteit Twente, op gezag van de rector magnificus,

prof. dr. H. Brinksma,

volgens besluit van het College voor Promoties

in het openbaar te verdedigen

op woensdag 30 september 2015 om 16.45 uur

door

\section{Dirk Jan Willem Dikken}

geboren op 5 januari 1986

te Amersfoort, Nederland 
Dit proefschrift is goedgekeurd door:

Prof. dr. J.L. Herek (Promotor) 


\section{Contents}

1 General introduction 1

1.1 Babinet's principle ................ 2

1.2 Optical antennas . . . . . . . . . . . . . . . . . 3

1.3 Resonance tuning . . . . . . . . . . . . . . . . . . . 7

1.3.1 Basic shapes ............ 8

1.3.2 Aspect ratio . . . . . . . . . . . . . . . . . . . 9

1.3.3 Coupling . . . . . . . . . . . . . . . . . 10

1.4 Antennas for Plasmons . . . . . . . . . . . . . . . 13

1.4.1 Surface Plasmon Polaritons (SPPs) . . . . . . . . . 13

1.4.2 Impedance of SPPs . . . . . . . . . . . . . . . 16

1.5 Outline of this thesis . . . . . . . . . . . . . . 18

2 Experimental techniques $\quad 21$

2.1 Dark-field micro-spectroscopy . . . . . . . . . . . . . . 21

2.2 Near-field microscopy . . . . . . . . . . . . . . . . . . . 26

2.2.1 Heterodyne detection . . . . . . . . . . . . . 27

2.2.2 Polarization resolved detection . . . . . . . . . . . 30

2.2.3 Shaped illumination ............ . . 32

I Antennas for Light 39

3 Width modulated antennas 41

3.1 Introduction . . . . . . . . . . . . . . . . . . . 41

3.2 Modulation phase . . . . . . . . . . . . . . . . 43

3.3 Modulation amplitude . . . . . . . . . . . . . . . 46

3.4 How to model the relation between shape and resonance? 49

3.4.1 Fredholm integral . . . . . . . . . . . . . . . 49

3.4.2 Oscillatory movement of an electron cloud . . . . . 50

3.4.3 Effective wavelength scaling . . . . . . . . . . . 52

3.4.4 The relation between E-fields and $\epsilon$ of an antenna 52

3.5 Asymmetric modulations . . . . . . . . . . . . . . 56

3.6 Conclusions . . . . . . . . . . . . . . . . . . . . . . 58

4 Coupling optical antennas $\quad 59$

4.1 Introduction . . . . . . . . . . . . . . . . . . 59

4.2 Coupled bar-ring system . . . . . . . . . . . . . . . 61

4.3 Dipole model . . . . . . . . . . . . . . . . . . . . . . . . 68 
4.4 Coupling of other two-antenna systems . . . . . . . . . . 72

4.5 Coupled Harmonic Oscillator model . . . . . . . . . . . . 74

4.5.1 From mechanical to optical . . . . . . . . . . . . . 75

4.5.2 Coupling of two loss-less mechanical oscillators . . 76

4.6 Conclusions ................. 78

II Antennas for Plasmons 81

5 Investigation of single hole-antennas $\quad 83$

5.1 Introduction . . . . . . . . . . . . . . . . 83

5.2 Transmission through sub-wavelength holes . . . . . . . . 85

5.3 Near-field distribution . . . . . . . . . . . . . . . . 89

5.3.1 Substrate material . . . . . . . . . . . . . . . . . . . . 90

5.3.2 Hole shape . . . . . . . . . . . . . . . . . . . . 91

5.4 Near-field measurement of a single hole . . . . . . . . . . 93

5.4.1 Near-field measurement of a single hole . . . . . . 94

5.4.2 K-space distribution of $E_{x, y}$ and $H_{x, y} \ldots \ldots . .9 .96$

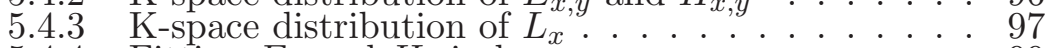

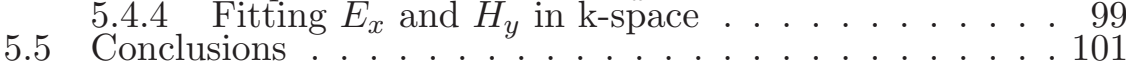

6 Active control of SPP fields 103

6.1 Introduction . . . . . . . . . . . . . . . . 103

6.2 Controlled generation of SPPs . . . . . . . . . . . . . . . 105

6.2.1 Amplitude and phase . . . . . . . . . . . . . 105

6.2.2 Controlling the polarization state . . . . . . . . . 108

6.3 Controlling the directionality of SPPs . . . . . . . . . . 110

6.3.1 Control by design . . . . . . . . . . . . . . . . . 110

6.3.2 Active control . . . . . . . . . . . . . 113

6.3.3 Manipulating the directivity of SPPS . . . . . . . . 114

6.4 Shaping light in the z-axis . . . . . . . . . . . . . 119

6.5 Circular polarized SPP fields . . . . . . . . . . . . . . . . 122

6.6 Conclusions . . . . . . . . . . . . . . . . . . . . . 127

A Fabrication techniques $\quad \mathbf{1 2 9}$

A.1 Electron-beam lithography . . . . . . . . . . . . . . . . 129

A.2 Synthesis of single crystalline Au flakes . . . . . . . . . . 131

A.3 Focused Ion Beam . . . . . . . . . . . . . . . . . . . 133

B Details on numerical calculations 137

C Modified coupled harmonic oscillator model 139

C.1 Single harmonically driven oscillator . . . . . . . . . . 139

C.2 The effects of loss . . . . . . . . . . . . . . . . . 140

$\begin{array}{ll}\text { D Reciprocity theorem } & 145\end{array}$

E Field distributions of holes with increasing diameter 149

Bibliography 153

$\begin{array}{ll}\text { Samenvatting } & 165\end{array}$

$\begin{array}{ll}\text { Dankwoord } & 169\end{array}$ 
Chapter 1

\section{General introduction}

Antennas have been used for over a century as emitters, scatterers and receivers of electromagnetic waves. All wireless communication devices, such as radio, mobile phones and satellite communication are strongly dependent on the capability of an antenna to localize propagating electromagnetic waves to a fraction of its wavelength. Because Maxwell's equations are scale-invariant the principles of antenna designs at radio frequencies $\left(10^{6} \mathrm{~Hz}\right)$ can also be applied at optical frequencies $\left(10^{14}\right.$ $\mathrm{Hz}$ ) [1]. Over the course of the last two decades, novel developments in nanotechnology have enabled the fabrication of antennas in the optical regime, and various optical antenna structures have been developed that have the potential to advance many light based technologies [2-5]. Using the efficient scattering of optical antennas, it has been shown for instance that the efficiency of solar cells can be increased by applying an antenna layer on top of the solar cell which effectively traps light in the solar cell [6]. In sensing applications improvements have been realized using the field localization that optical antennas enable [7-9]. The power of optical antennas lies in their capability to manipulate the light at the nanoscale. This freedom of controlling light at the nanoscale is very sensitive to shape and material of the antenna and its surroundings.

In this thesis, we study two types of antennas: antennas for light and antennas for surface bound waves called an surface plasmon polaritons (SPPs). The antennas for light we consider in this thesis are one or two small metal particles, and we will be mostly concerned with how they scatter light that impinges on them and with how this is influenced by their geometry. The antennas for SPPs we consider here are simple round holes in a metal film, each hole acts as a source for surface waves and by controlling the impinging light and the positions of the holes, we 
control the fields on the surface. In both antenna types, charge density waves play an important role in the antennas for light the waves are localized to a metallic particle, while in the antenna for SPPs, propagating charge density waves are generated. We study the far- and near-fields of these two types of antennas both experimentally and theoretically. For the antennas for light we are able to unravel and manipulate several of the parameters which determine the resonance behavior of single and coupled antenna systems. For the antennas for plasmons we show that we can actively control the polarisation and phase of each antenna, and thereby create tailored surface plasmon fields.

This Chapter provides a general introduction into nano-optics and optical antennas. First, we describe the link between antennas for light and antennas for SPPs using Babinet's principles. Whereafter an analytical description of a spherical antenna highlights the basic concepts of optical antennas and illustrates what parameters determine their resonance wavelength. We focus on three geometrical parameters which influence the resonance behavior of optical antennas. Next, we discuss various aspects of surface plasmon polaritons (SPPs), which are generated when light propagates through hole-antennas. Finally, we conclude this Chapter with an outline of this thesis.

\subsection{Babinet's principle}

The two types of optical antennas which we study are depicted in Figure 1.1, where a hole in a metallic film and a complementary shaped metallic particle are shown. At first glance, metallic particles and holes in metallic films do not have that much in common. Babinet's principle, however, shows us that the scattering properties of optical antennas and those of holes in metallic films can be related to one another. Babinet's principle relates the diffraction fields of a diffracting screen having an aperture $\left(S_{A}\right)$ with those of a complementary screen $\left(S_{B}\right)$, see Figure 1.1. A relation between EM fields of the diffracting screen and its complement can be derived, using Babinet's principle, when assuming the screen and the optical antenna are made from a perfect conducting material. Babinet's principle states that the intensity of the diffraction pattern of the screen and its complement will be the same, and that the fields are related by [10]:

$$
\begin{aligned}
\mathbf{E}_{\text {aper }} & =-\mathbf{H}_{\text {ant }} \\
\mathbf{H}_{\text {aper }} & =\mathbf{E}_{\text {ant }}
\end{aligned}
$$


where $\mathbf{E}_{\text {aper, ant }}$ and $\mathbf{H}_{\text {aper, ant }}$ indicate the electric and magnetic fields of an aperture and antenna, respectively. The vector formulation of Babinet's principle can be very useful in the design of optical antennas and aperture systems, as it states that the electric and magnetic fields of an aperture are equal to the magnetic and electric components of an antenna, respectively. Although most metals at optical frequencies cannot be described as perfect conductors, the basic concept of Babinet's principle still holds and has been applied to explain the optical behavior of apertures antennas [11-13]. Even though Babinets's principle is not strictly valid for the real metals we are interested in, Figure 1.1 still nicely portrays the two types of systems we study in this thesis: light impinging on the aperture thereby generating surface plasmons propagating over the metal sheet and, light impinging on the particle generating localized surface plamons.
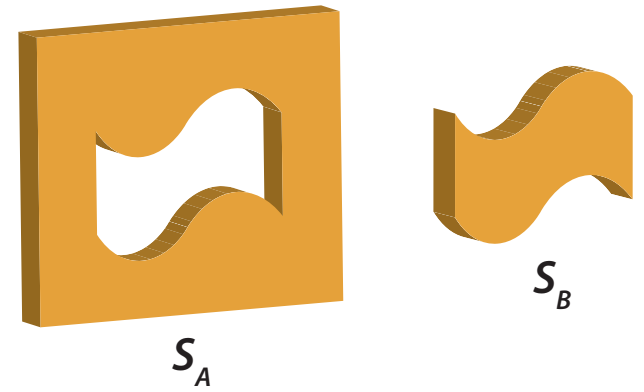

Figure 1.1: Babinet's principle. (Left) perfect conducting sheet with an aperture of arbitrary shape $\left(S_{A}\right)$, (Right) shows the antenna shape $\left(S_{B}\right)$ which is the complimentary shape of $\left(S_{A}\right)$.

\subsection{Optical antennas}

Antennas are widely used for their capability of transferring localized energy to free-space radiation and vice-versa. Antenna concepts which have been used in the radio and microwave regime, can often also be applied to optical antennas. Due to their subwavelength dimensions, optical antennas enable the control and localization of optical fields at the nanometer scale, illustrated in Figure 1.2, showing great promise for enhancing the performance of many optical devices in sensing $[7,9,14]$, light emission $[15,16]$, electronics [17-19] and photovoltaic [6] applications. 


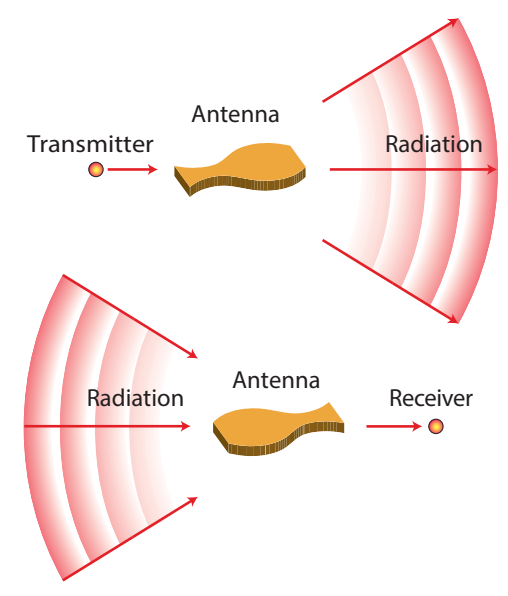

Figure 1.2: Working principal of an antenna. (A) transmitting antenna and (B) receiving antenna. In this thesis a variety of optical antenna shapes are discussed. Image adapted from [20].

Optical antennas are metal structures which contain free-electrons, and like conventional antennas, the free-electrons present in the metal can easily be driven by an external field. In turn, the optical antenna can also emit light, as the movement of the free electrons produces a dipole moment and an oscillating electric field, leading to the emission of free-space radiation. Both in conventional and in optical antennas only the free electrons within the skin-depth of the metal can be driven by an external field. Where the skin-depth in conventional and optical antennas is in the order of $20-30 \mathrm{~nm}$, the physical dimensions of antennas in both frequency regimes, with relation to the skin-depth, is dramatically different. This means that for conventional antennas only a very small fraction of the free electrons participate, while, due to their subwavelength dimensions, the free electrons of the whole volume of an optical antenna participate. In the optical regime, the penetration of light into metals can no longer be neglected, and the finite electron density in optical antennas results in a delay between the driving field and the electronic response of the antenna. This means is that the skin-depth of optical antennas is often larger than the diameter of the antenna, which has been shown to lead to the effect where thin optical antennas do not respond to the wavelength $\lambda$ of the incident radiation, but to an effective wavelength $\lambda_{\text {eff }}$ [20-22] (see Section 1.3.2).

In Chapter 3 we will look into the shape dependence of the resonance wavelength of an antenna, and show that the shape of an antenna can be tuned to the extreme, where the dimensions of the antenna are 13 
times smaller than their resonance wavelength. The resonance behavior of simple antenna shapes, like spheres, ellipsoids and rods, can be analytically described, however almost all other antenna shapes are already too complex, and require rigorous numerical calculations. Numerical techniques like FEM, FIT and FDTD, are often used to calculate the near-field distributions and resonance behavior of antennas.

In order to obtain a better understanding of the plasmon resonances present in optical antennas, let us now consider the excitation of a small spherical particle with radius $a$ in the quasi-static approximation. The quasi-static approximation essentially means that we assume that the particle is significantly smaller than $\lambda$, and no retardation effects are taken into account. Using the Laplace equation the electrostatic potential of a sphere can be derived, with which the fields inside and outside the sphere are given (shown in spherical coordinates $(\mathrm{r}, \theta, \phi))[23]$ :

$$
\begin{gathered}
E_{\text {in }}=E_{0} \frac{3 \epsilon_{d}}{\epsilon_{m}+2 \epsilon_{d}}\left(\cos (\theta) \mathbf{n}_{r}-\sin (\theta) \mathbf{n}_{\theta}\right) \\
E_{\text {out }}=E_{0}\left(\cos (\theta) \mathbf{n}_{r}-\sin (\theta) \mathbf{n}_{\theta}\right)+\frac{\alpha}{4 \pi \epsilon_{0} r^{3}} E_{0}\left(2 \cos (\theta) \mathbf{n}_{r}+\sin (\theta) \mathbf{n}_{\theta}\right)
\end{gathered}
$$

where $\epsilon_{m, d}$ are the dielectric constant of the metal (antenna) and the dielectric surrounding the antenna, and $\alpha$ describes the polarizability of the antenna:

$$
\alpha(\omega)=4 \pi \epsilon_{0} a^{3} \frac{\epsilon_{m}(\omega)-\epsilon_{d}}{\epsilon_{m}(\omega)+2 \epsilon_{d}}
$$

The first and the second term in Equation 1.3 describe the electric field of the incident field, and the field which is scattered by the particle, respectively. The scattered field from the particle is identical to the field of a dipole, which is situated in the center of the particle, having a dipole moment $\boldsymbol{p}=\epsilon_{2} \alpha(\omega) \mathbf{E}_{0}$. The spherical particle is at resonance when $\left|\epsilon_{m}(\omega)+2 \epsilon_{d}\right|$ is minimal and $\alpha$ increases to a maximum. At resonance, the local fields of the antenna, second term of Equation 1.3, strongly increase and scale with the polarizability. The strength with which antennas are able to absorb or scatter light can be described by their absorption $\left(\sigma_{a b s}\right)$ and scattering $\left(\sigma_{\text {scat }}\right)$ cross sections. The scattering cross section $\left(\sigma_{\text {scat }}\right)$ of the sphere is obtained by dividing the total radiated power of the sphere's dipole by the intensity of the plane wave. Similarly the power which is removed from the incident beam due to the presence of an antenna, can be described by the extinction cross section $\left(\sigma_{\text {ext }}\right)$, which is the sum of $\left(\sigma_{a b s}\right)$ and $\left(\sigma_{\text {scat }}\right)$. For the spherical particle 
one can analytically derive $\left(\sigma_{a b s}\right)$ and $\left(\sigma_{\text {scat }}\right)[23]$ :

$$
\begin{gathered}
\sigma_{a b s}=\frac{k_{0}}{\epsilon_{0}} \operatorname{Im}(\alpha(\omega)) \\
\sigma_{\text {scat }}=\frac{k_{0}^{4}}{6 \pi \epsilon_{0}^{2}}|\alpha(\omega)|^{2}
\end{gathered}
$$

These equations nicely illustrate that the ratio with which light is absorbed and scattered by the antenna strongly depends on the dimensions of the antenna. The absorption scales with $a^{3}$ and the scattering scales with $a^{6}$. Consequently for large particles the extinction is dominated by scattering while smaller particles mainly absorb light. Figure 1.3 shows the scattering and absorption cross sections of an $\mathrm{Au}$ metallic sphere having a radius which varies between 100 and $150 \mathrm{~nm}$. The $\left(\sigma_{a b s}\right)$ and $\left(\sigma_{\text {scat }}\right)$ show two clear resonance peaks which are located at different wavelengths, meaning that the metallic particle will strongly scatter green light and strongly absorb yellow light. With increasing particle radius, Figure 1.3 nicely illustrates how the ratio of the resonance peaks of $\left(\sigma_{a b s}\right)$ and $\left(\sigma_{\text {scat }}\right)$ vary from being dominated by absorption to being dominated by scattering.

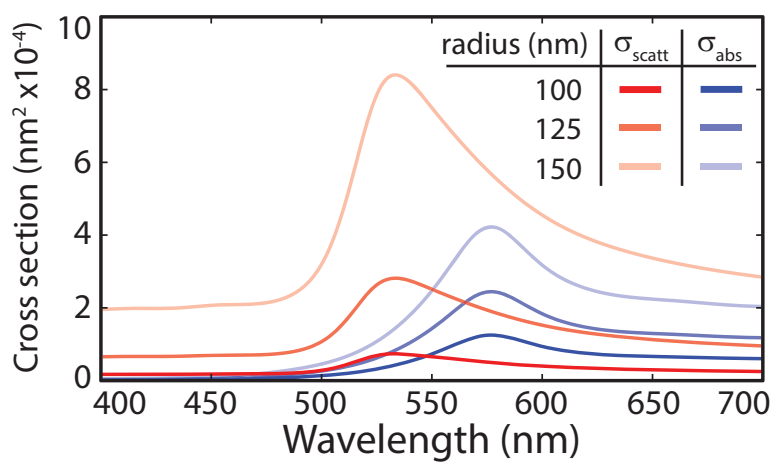

Figure 1.3: Scattering and absorption cross section of metal nanospheres. Scattering $\left(\sigma_{\text {scatt }}\right)$ and absorption $\left(\sigma_{a b s}\right)$ cross sections of spherical gold particles with a radius of 100,125 and $150 \mathrm{~nm}$, which are surrounded by a dielectric medium with $\epsilon=1$. 


\subsection{Resonance tuning}

In many applications, optical antennas are used for their ability to localize light to small volumes, where the intensity can be several orders of magnitude larger than the incident light [24,25]. The strong field localization is a property of optical antennas which is often used to enhance linear and non-linear processes [26-31]. Especially non-linear processes benefit from increased field strengths as for instance second- and thirdharmonic generation scale with the $|E|^{2}$ and $|E|^{3}$, respectively. Although for non-linear effects to occur, also phase matching conditions have to be satisfied.

\section{Basic shapes}

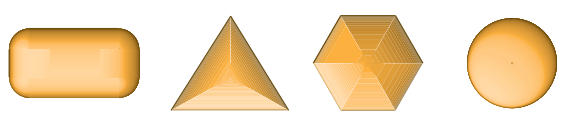

\section{Aspect ratio}

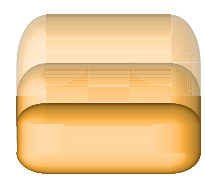

\section{Coupling}

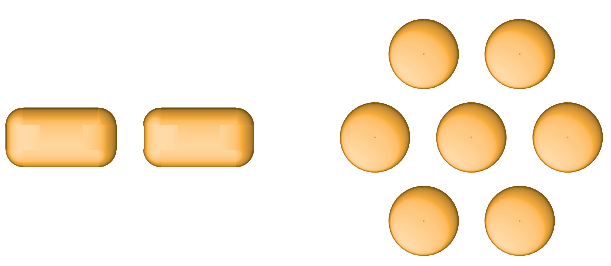

Figure 1.4: Factors which affect the resonance wavelength of an antenna. Three groups of cartoons, showing examples of widely used approaches to tune the resonance wavelength of optical antennas.

As can be seen from Figure 1.3, to maximize the optical response and the resulting electric fields of an optical antenna, one has to drive the antenna at its resonance wavelength. The resonance wavelength of the optical antenna therefore is an important tuning factor to maximize the linear and non-linear interaction between the fields of the antenna and the medium of interest. The relation between shape and resonance wave- 
length is still actively studied, as there is a strong and partially unknown connection between the two. However, in other optical applications controlling the resonance wavelength also plays a dominant role. A nice example of this is the use of a variety of v-shaped optical antennas to make optical phase plates [32]. In this section we will briefly discuss three aspects with which the resonance wavelength of optical antennas can be tuned, as illustrated in Figure 1.4.

\subsubsection{Basic shapes}

Optical antennas with various geometrical shapes have been studied over the years [33-36], varying from: spheres [4], ellipsoids [37,38], disks [39], triangles [40], cubes [41], wires [42], crescents [43], stars [44] and many more. In most cases when optical antennas are small (dimensions $\ll \lambda$ ), the antenna exhibits a dipole-like radiation pattern, regardless of shape. Butet et al. [45] have shown that despite large geometrical deformations and asymmetry, giving rise to one or multiple local anti-phased charge distributions, the overall far field radiation pattern is dipolar. This is an interesting finding, as it suggests that while local anti-phased charge distributions might have a large effect on the spectral dependence of a small antenna they have little or no effect on the far-field radiation pattern. In Section 3.4 we discuss the applicability of several models, which are often used to describe the resonance behavior of antennas which show strong dipolar resonances and exhibit local charge distributions which oscillate in anti-phase.

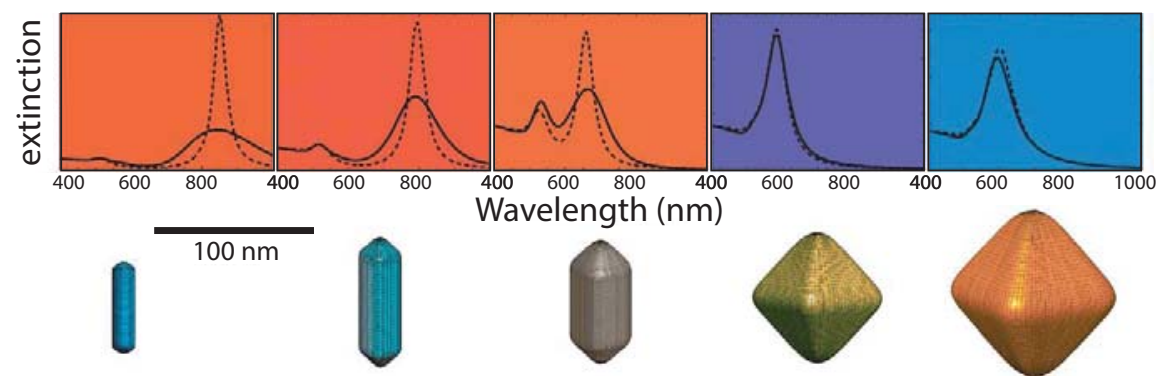

Figure 1.5: Effect of shape on the resonance wavelength of an optical antenna. The relation between shape and resonance wavelength is investigated experimentally (solid line) and numerically (dashed line) for a rod and an octahedra shaped antenna, and three intermediate forms. Image is taken from [46]. 
Figure 1.5 exemplifies how the resonance behavior of an optical antenna is determined by the shape of the antenna, where the extinction spectra of a rod and octahedra shaped antenna, and three intermediate antenna shapes are shown. The different antenna shapes are chemically synthesized and measured in bulk in solution. The numerically obtained extinction curves are calculated with a boundary element method (BEM) [47]. The difference between the experimental data and theory is a common problem, which is associated with the fact that in the experiment a solution of particles is used, which in practice slightly vary in shape and size, leading to broadened spectra. For this reason we have developed an experimental setup which is capable of obtaining scattering spectra of individual optical antennas, which have dimensions down to $50 \mathrm{~nm}$ Section 2.1. The extinction spectra, which are taken from [46], show a blue shift for the rod antenna as it transforms into an octahedra antenna. The single longitudinal mode of the rod antenna transforms into a more symmetric mode of the octahedra antenna, while passing through the two plasmon modes of the intermediate low-aspect-ratio rods.

\subsubsection{Aspect ratio}

A simple view on the shape dependence of the resonance, can be gained by comparing the oscillating electron density waves, which are present on an optical bar antenna, to light propagating in a Fabry-Perot cavity $[23,48]$, which is illustrated in Figure 1.6. With this model it is possible to derive the resonance behavior of simple bar antennas. In the Fabry-Perot approach, the cavity/antenna is at resonance when the phase accumulated in one round trip is equal to $n \pi(n=1,2,3, \ldots)$. Components which attribute to the accumulated phase are the real part of the propagation constant $(\gamma)$ of the electron density waves $(\beta)$, which propagate along the bar antenna, and an additional phase $\left(\Phi_{R}\right)$ which is picked up at the end-caps of the antenna, so that:

$$
\beta L_{r e s}+\Phi_{R}=n \pi
$$

where $L_{\text {res }}$ is the length of the rod when it is at resonance. For decreasing wire radius or increasing aspect-ratio, $\beta$ rapidly increases, implying that the length, which is needed for the antennas to be resonant, decreases, as:

$$
L_{r e s}=\frac{n \pi-\Phi_{R}}{\beta}
$$

A clear linear relation between the length of the antenna $\left(L_{r e s}\right)$ and the wavelength $1 / \beta=\lambda / 2 \pi$ is shown in Equation 1.8. Novotny shows in [49] 


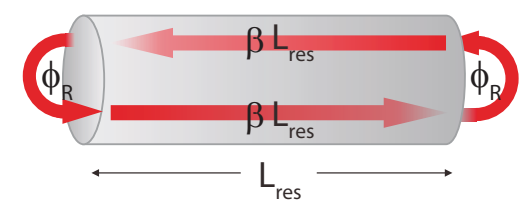

Figure 1.6: Cartoon depicting the Fabry-Perot model applied on an optical model. The resonance of the bar antenna is modeled as a Fabry-Perot cavity where the SPPs of the optical antenna represent the beams of light, which propagate in the cavity. At the end-caps an additional phase $\left(\Phi_{R}\right)$ is acquired. Image is taken from [48].

that high aspect-ratio antennas do not respond to the wavelength $\lambda_{0}$ of the incident radiation, but to an effective wavelength $\lambda_{\text {eff }}=a+b \lambda_{0}$. Although this model provides an intuitive scaling relation between the aspect-ratio of an antenna and its resonance wavelength, this model still contains parameters, $a$ and $b$, which can vary for different antenna shapes. This means that this model does not provide a generalized relation between the shape of an antenna and its resonance behavior. The Fabry-Perot model retains its validity as long the propagation constant $\beta$ is well defined within the shape of the antenna. If the antenna shape is wildly modulating within the space between the end-caps, $\beta$ is ill defined and the Fabry-Perot model starts to fail. Studies on different shapes of the end-caps of bar antennas have revealed that the phase shift $\Phi_{R}$ can be modified such that it becomes negative, leading to a zero-order resonance, where $n=0[50,51]$. This yet again shows that relatively small changes in the shape of an antenna can lead to significant changes to its resonance wavelength.

\subsubsection{Coupling}

When two antennas are positioned close together and are illuminated by a harmonic driving field, in literature these antennas are often referred to as being 'coupled'. As it is not immediately clear what is meant by this, we discuss our view of the term 'coupling'. In Section 1.2, we found that the antenna itself generates an electric field as a result of it being driven by an external field. If now two antennas are positioned close together, the generated electric field of one antenna will also affect the driving of the free electrons in the second antenna, and vice versa. In the simplest case, where two dipole antennas are positioned close together, the coupled system has two eigen-states where the antennas resonate in-phase (symmetric) and out-of-phase (anti-symmetric), which is illustrated in Figure 1.7. When the two antennas strongly interact, 

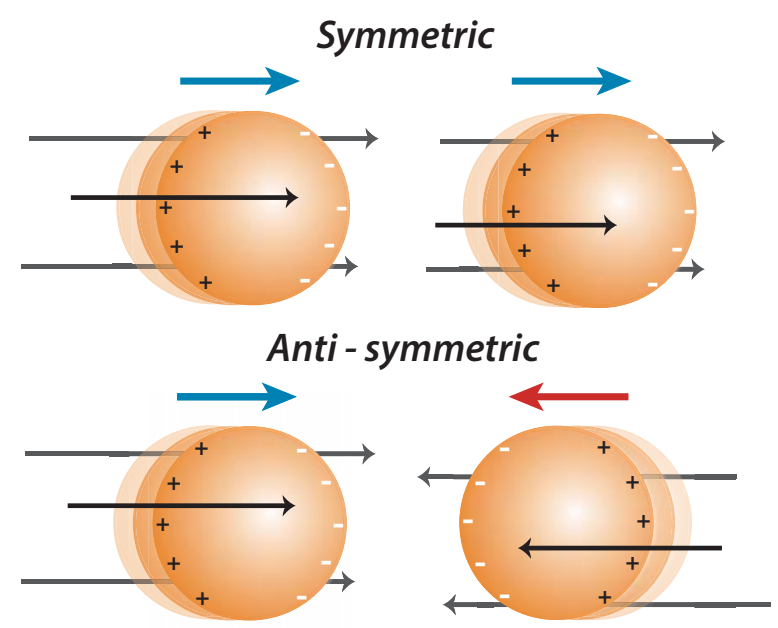

Figure 1.7: Illustration of the oscillatory movement of two coupled oscillators. The oscillatory movement of two free electron clouds, moving in a symmetric and anti-symmetric fashion, indicated with the blue and red arrows.

the resulting eigenmode of the coupled system will show spectral splitting, similar to the spin-spin interaction of the orbitals of atoms in a molecule, where the symmetric and anti-symmetric eigenmodes are redand blueshifted, respectively.

The anti-symmetric eigenmode of the coupled system is often referred to as being a dark mode; the excitation of this mode is symmetryforbidden for plane wave excitation under normal incidence [52]. The anti-symmetric eigenmode can be excited when the structure is illuminated under an oblique angle, or if the symmetry of the shape of the antenna is broken [53,54]. Another way to study the resonance of optical antennas is to study their two-photon excited photoluminescence (TPPL), which strongly increases when the local fields are enhanced whenever the antenna is resonantly excited. With this technique it is possible to break the symmetry, by scanning a strongly focused spot over the antenna making it possible to visualize the anti-symmetric coupling mode [55].

The spectral distribution of two coupled optical antennas can be understood by modeling the antennas as two harmonically driven coupled mechanical oscillators [56-60]. The oscillatory movement of the free electron cloud of an optical antenna is modeled as a mass-spring resonator. Figure 1.8 shows the configuration where two mechanical oscillators are 


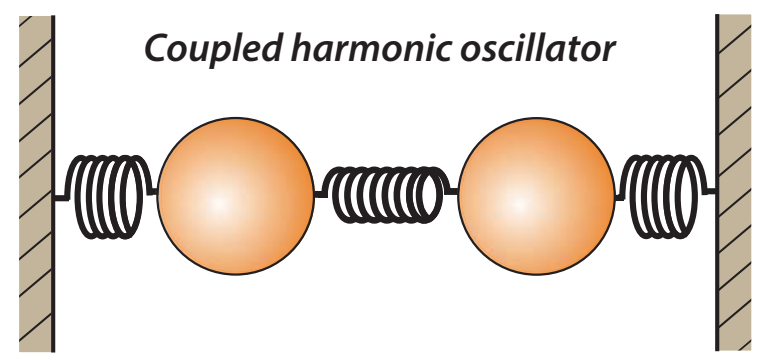

Figure 1.8: Coupled harmonic oscillator model. Schematic representation of the coupled harmonic oscillator model, consisting of two mass-spring oscillators and a third coupling spring.

coupled with a coupling spring. In Chapter 4 we discuss the coupling of two optical antennas having different polarizability, and we use the mechanical oscillator model approach to describe their resonance behavior and far-field radiation pattern. The mechanical oscillator model implicitly assumes that the two eigenmodes of the coupled system can be described as a linear combination of the two individual antennas. In this approach, it is thus assumed that the mode profiles / charge distributions of the two antennas are not changed, and that only their relative amplitude and phase are changed when the antennas are positioned close together.

A very similar model, which approaches the coupling of optical antennas from a chemical perspective is called the hybridization model. This model gives an intuitive picture of plasmon resonances of complex antennas/antenna systems, where plasmon resonances are described as a 'hybridization' of the resonances of the substructures from which the antenna is formed [61]. In Figure 1.9 (A) we give an example, where

A

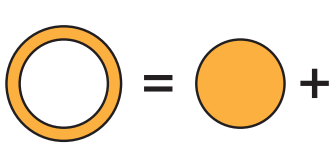

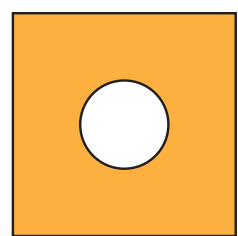

B

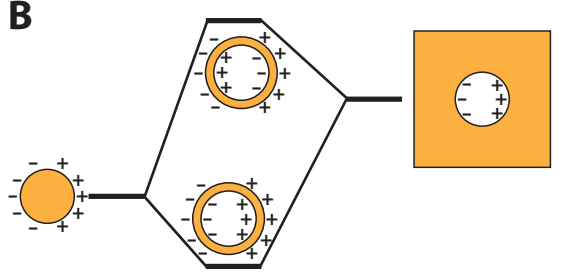

Figure 1.9: Hybridization model. (A) elementary structures. (B) schematic representation of the energy levels of the elementary and hybridized modes, where the charge distribution, are indicated with plus and minus signs. Images adapted from [23]. 
the resonance of a hollow metallic shell is reconstructed from two elementary shapes: consisting of a metallic sphere and a spherical cavity in bulk metal [23]. Figure 1.9 (B) shows that a low-energy hybrid mode is obtained when the elementary modes oscillate in-phase, meaning that the charge distribution inside and outside the shell oscillate in phase. Consequently a strong dipole moment is formed. A high-energy hybrid mode is formed when the elementary modes oscillate out-of-phase, and the charge distribution inside and outside the shell oscillate out-of-phase. The dipole moment of this mode is therefore weak. This line of reasoning also holds for calculating the resonance behavior of multiple antennas, making it possible to calculate the spectral behavior of complex antenna systems, based on the resonance behavior of the basic antenna shapes from which they are formed.

\subsection{Antennas for Plasmons}

Several antenna configurations have been explored in order to generate SPPs on a metal dielectric interface, varying from holes of different shapes and sizes, which have been made in a metal film, to small metal particles, which are placed on top of a metal film [11,62-64]. In this thesis we are interested in studying and eventually controlling the nearfield distributions, which surround the various hole-antenna geometries.

\subsubsection{Surface Plasmon Polaritons (SPPs)}

Surface plasmon polaritons are charge density waves, which manifest at the interface between a dielectric and a metal. They are evanescent in both the perpendicular directions from the surface. The most simple geometry sustaining SPPs is that of a single, flat interface between a dielectric and a metal, where the SPPs are transverse magnetic (TM), as illustrated in Figure 1.10 (A). The charge density waves, which are indicated with the plus and minus signs in the metal, lead to the field distribution indicated with red lines. The wavevector of the SPPs traveling along the interface, is related to the optical frequency $\omega$ via the dispersion relation [65]:

$$
k_{x}=k_{0} \sqrt{\frac{\epsilon_{d} \epsilon_{m}}{\epsilon_{d}+\epsilon_{m}}}
$$

where $k_{0}$ describes the wavevector of free-space emission, and $\epsilon_{d}$ and $\epsilon_{m}$ the dielectric constants of the dielectric and metal medium, respectively. In this dispersion relation we assume that the interface is normal to $\hat{\mathbf{z}}$ and the SPPs propagate along the $x$ direction. The normal components 
of the wavevector into the medium $\left(\epsilon_{d, m}\right)$, is given by [65]:

$$
k_{z}=k_{0} \sqrt{\frac{\epsilon_{d, m}^{2}}{\epsilon_{d}+\epsilon_{m}}}
$$

Now that we have obtained an expression for the in- and out-of-plane dispersion relations of a confined interface mode, we discuss the conditions which have to be fulfilled in order for a surface mode to exist [23]:

$$
\begin{aligned}
\epsilon_{m}(\omega) \cdot \epsilon_{d}(\omega) & <0 \\
\epsilon_{m}(\omega)+\epsilon_{d}(\omega) & <0 .
\end{aligned}
$$

This means that one of the dielectric constants must be negative, and have a larger absolute value than the other. Metals and especially noble metals show a larger negative real, and a small imaginary dielectric constant for frequencies in the optical regime. At the interfaces of a noble metal, like gold or silver, and a dielectric, like glass or air, the surface modes can thus exist.
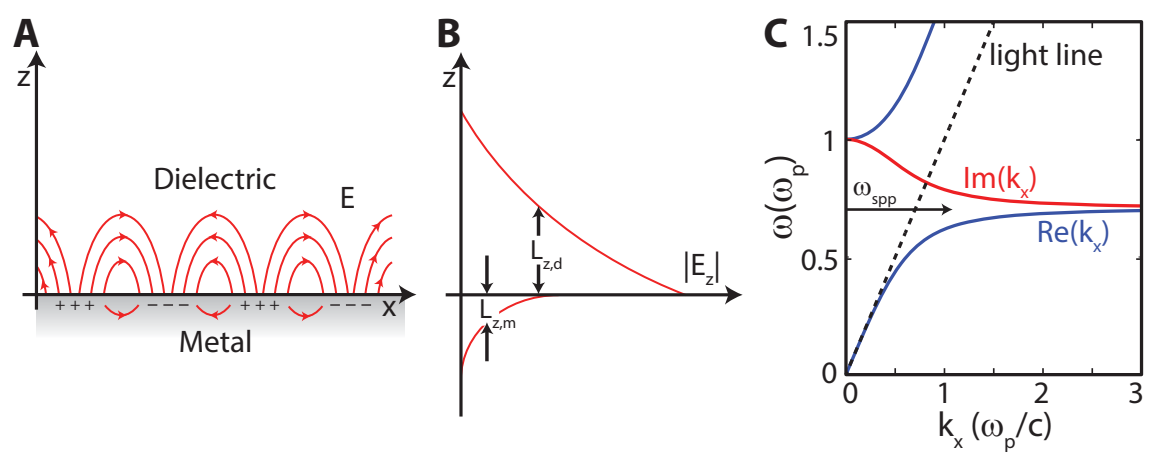

Figure 1.10: Surface plasmon polaritons. (A) cartoon depicting the charge density oscillations and electromagnetic field distributions, which are associated with SPPs. The charge distribution is indicated with the plus and minus signs in the metal. (B) cartoon depicting the exponentially decaying out of plane electric field component in the dielectric and the metal. The decay $(1 / e)$ length in the dielectric and the metal are indicated with, $L_{z, d}$ and $L_{z, m}$, respectively. (C) cartoon depicting the dispersion of SPPs at a lossless Drude metal-air interface. Images A, B and $\mathbf{C}$ where adapted from [65] and [66], respectively.

The optical properties of metals are often expressed in terms of the Drude model, which describes the optical response of metals as a free electron gas [23]:

$$
\epsilon_{\text {Drude }}(\omega)=1-\frac{\omega_{p}^{2}}{\omega^{2}+i \Gamma \omega}
$$


where $\omega_{p}$ is the plasma frequency. The motion of the electron gas is damped via collisions, which eventually heat up the metal; the damping is described with the collision frequency $\Gamma$. The oscillatory motion of the free electrons in the metal is simplified as the mechanical motion of a mass-spring system, and as such the interband transitions which occur in real metals are not taken into account. Because of this, the Drude model reaches its limit in describing the optical behavior of metals at lower wavelengths where interband transitions occur. However, the Drude model is still very useful to understand the basic behavior of SPPs.

Figure 1.10 (C) shows the dispersion curve of Equation 1.12 for SPPs at a lossless Drude metal-air interface $\left(\Gamma=0\right.$ and $\left.\epsilon_{\text {air }}=1\right)$. For $\omega<\omega_{S P P}$, the SPP wavevector is larger than $k_{0}$ and the dispersion curve lies below the light line, indicated with the black dashed line. Because of the momentum mismatch between SPPs and free-space emission, it is impossible to directly excite SPPs using free-space emission. Alternative methods, like gratings, hole-antennas or metal particles on the metaldielectric surface are needed to overcome this momentum mismatch.

Figure $1.10(\mathrm{C})$ also shows that $k_{x}$ is purely imaginary for $\omega_{S P P}<\omega<$ $\omega_{p}$, which means that on the interface no propagating SPP mode is present, but rather a mode which is exponentially decaying. In the case that $\omega>\omega_{p}$ the metal is transparent, meaning that the frequency of the driving field is too high for the electrons in the metal to follow. At $\omega=\omega_{S P P}$ the wavevector diverges in the case of a lossless Drude metal, which is not the case for a real metal, where the dispersion curve bends back.

The Ohmic losses in a medium are associated with the imaginary part of the dielectric function: $\epsilon_{m}=\epsilon_{m}^{\prime}+i \epsilon_{m}^{\prime \prime}$, where $\epsilon_{m}^{\prime}$ determines the wavelength and $\epsilon_{m}^{\prime \prime}$ accounts for the damping of light propagating in the medium. Assuming that $\left|\epsilon_{m}^{\prime \prime}\right| \ll\left|\epsilon_{m}^{\prime}\right|$, the SPP wavelength is given by:

$$
\lambda_{S P P}=\frac{2 \pi}{k_{x}^{\prime}} \approx \sqrt{\frac{\epsilon_{m}^{\prime}+\epsilon_{d}}{\epsilon_{m}^{\prime} \epsilon_{d}}} \lambda_{0}
$$

where $\lambda_{0}$ is the wavelength in vacuum. The length over which the amplitude decays by a factor of $1 / e$, also known as the propagation constant, is given by $L_{S S P}=1 / k_{x}^{\prime \prime}$. As an example, an amplitude propagation length of the SPPs on a Au-air interface of $\approx 175 \mu \mathrm{m}$ is found where the experimental values of $\epsilon_{A u}=-40.3+2.8 i$ at $\lambda=950 \mathrm{~nm}$ are used [67]. 
The decay length of the SPP fields, in the medium $\epsilon_{j}$ perpendicular to the interface, is:

$$
L_{z, j}=\frac{1}{k_{z, j}}=\frac{1}{k_{0}} \frac{\sqrt{\epsilon_{d}+\epsilon_{m}^{\prime}}}{\epsilon_{j}^{\prime}}
$$

The decay length into the dielectric scales approximately with $1 / \epsilon_{d}$ when $\epsilon_{d} \ll \epsilon_{m}$, which is exemplified when we calculate the decay length of SPPs on an Au-air and an Au-glass interface where $L_{z, d}=98$ and 43 $\mathrm{nm}$, respectively. In both cases, the decay length into the metal $\left(L_{z, m}\right)$ is constant at $24 \mathrm{~nm}$. The ratio of the decay lengths into the dielectric and metal is illustrated in Figure 1.10 (B).

\subsubsection{Impedance of SPPs}

In free-space the relative magnitude of the electric and magnetic field components are related by the vacuum impedance:

$$
Z_{0}=\frac{E}{H}=c \mu_{0}
$$

where $\mathrm{c}$ is the speed of light and $\mu_{0}$ the free-space permeability, so that $Z_{0}=376.7 \Omega$. This relation can be derived using the Maxwell equation of the curl of $\mathrm{E}$ :

$$
\mathbf{k} \times \mathbf{E}=\mu_{0} \omega \mathbf{H}
$$

Now we set out to calculate the impedance, and thus the ratio of the $\mathrm{E}$ and $\mathrm{H}$ fields, of SPPs on a Au-air interface. Obtaining a better understanding of the ratio of the $\mathrm{E}$ and $\mathrm{H}$ fields, which are associated with SPPs on a Au-air interface, is, as will be shown in Chapters 5 and 6, of crucial importance in order to understand the experimental near field microscope (NSOM) signals. In Appendix D the reciprocity theorem is explained, which shows that the signals obtained with the NSOM contain a mixture of both in-plane electric $\left(E_{x, y}\right)$ and magnetic $\left(H_{y, x}\right)$ components. We chose a coordinate system in which $\mathbf{E}, \mathbf{H}, \mathbf{k}$ point in the $\mathrm{x}, \mathrm{y}$ and z-direction, respectively. The electric fields of an SPP on the $\mathrm{Au}$-air interface are given by [23]:

$$
\mathbf{E}=E_{x}\left(\begin{array}{c}
1 \\
0 \\
-k_{x} / k_{z}
\end{array}\right) e^{i k_{z} z}
$$

where $k_{x}$ and $k_{z}$ are given by Equations 1.9 and 1.10, respectively. By inserting the derived field distribution of Equation 1.17 in Equation 1.16 
we obtain:

$$
\left(\begin{array}{c}
k_{x} \\
0 \\
k_{z}
\end{array}\right) \times E_{x}\left(\begin{array}{c}
1 \\
0 \\
-k_{x} / k_{z}
\end{array}\right) e^{i k_{z} z}=\mu_{0} \omega \mathbf{H}
$$

so that in-plane electric $\left(E_{x}\right)$ and magnetic $\left(H_{y}\right)$ field components are related by:

$$
E_{x}=\frac{\sqrt{\epsilon_{d}+\epsilon_{m}}}{c \mu_{0}} H_{y}
$$

This equation is equally valid when comparing $\left(E_{y}\right)$ and $\left(H_{x}\right)$ and we find the SPP impedance for the in-plane field components on an Au-air interface:

$$
Z_{S P P}=\frac{E_{x}}{H_{y}}=Z_{0} \frac{1}{\sqrt{\epsilon_{d}+\epsilon_{m}}}
$$

The impedance of the SPPs is a complex number, which is wavelength dependent, as it is based on the dielectric constant of $\epsilon_{m}$. Figure 1.11 shows the impedance of the SPPs on an Au-air interface as a function of wavelength, for a wavelength of $950 \mathrm{~nm}\left|Z_{\text {spp }}\right| \approx 0.16 Z_{0}$. This result shows that for SPPs the in-plane magnetic field component is larger compared to the free-space light. Note that the value is dominated by the imaginary part, and thus has a phase of approximately $-\pi / 2$, this shows that in a propagating SPP the $E_{x}$ and $H_{y}$ fields are about $\pi / 2$ out of phase.
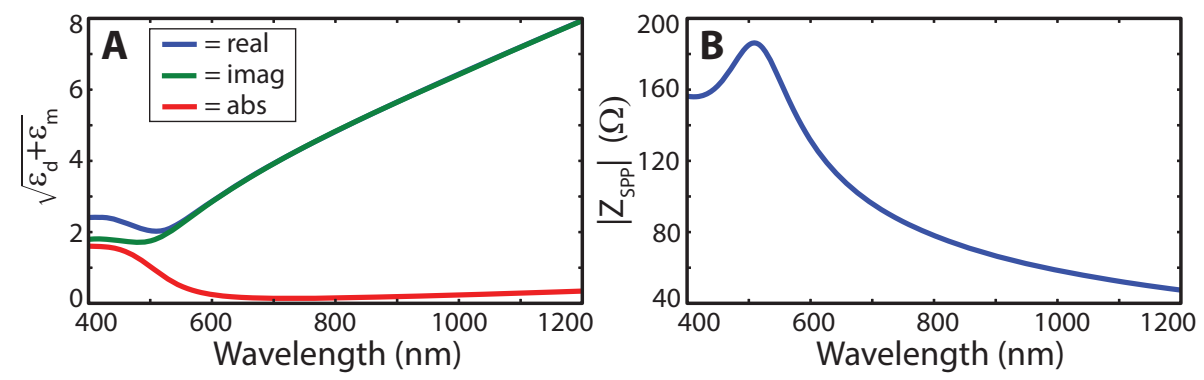

Figure 1.11: The impedance of the SPP on an Au-air interface. (A) the real, imaginary and absolute part of $\sqrt{\epsilon_{d}+\epsilon_{m}}$. (B) shows the $Z_{S P P}$ which provides a measure for the ratio of the in-plane electric and magnetic fields of the SPPs on an Au-air interface. 


\subsection{Outline of this thesis}

In this thesis, we experimentally and numerically study the far-field and near-field distributions of optical antennas, and show how the resonance characteristics and directionality can be tuned by varying their geometry and/or the illumination conditions.

In Chapter 2 we introduce two experimental techniques, which are used to characterize the far-field spectral behavior and the near-field distribution of optical antennas. A dark-field micro-spectrometer is described, with which it is possible to characterize the spectral behavior of a single antenna within a spectral range of 400-1000 nm. In the second part, we introduce an aperture type near-field scanning optical microscope (NSOM), used to study the near-field vector fields near optical antennas and to manipulate the amplitude, phase and polarization state of the illumination light. We explain the working principles of this microscope, and how we can measure the near-field amplitude, phase and polarization state at any point in space. Finally, we discuss the implementation of an spatial light modulator (SLM) into the NSOM, so that we are able to the shape the illumination conditions (amplitude, phase and polarization state) for every antenna.

\section{Part I - Antennas for Light}

In Chapter 3 the spectral dependence on antenna shape is studied using a sinusoidally width-modulated antenna. By simply varying the phase of the modulation, a smooth transition between a convex and concave antenna shape is realized, leading to extreme resonance shifts of up to $600 \mathrm{~nm}$. We discuss the applicability of several often used engineering models, which relate certain aspects of the antenna shape to their resonance wavelength.

In Chapter 4 we experimentally and numerically study how the resulting eigenmodes of a coupled system are affected when two optical antennas, showing different degrees of polarizability, are coupled. A coupled harmonic oscillator model is used in which a mechanical equivalence for the optical polariability of an antenna is implemented, making it possible to explain the spectral behavior and angle-dependent emission of coupled optical antennas having different degrees of polarizability. 


\section{Part II - Antennas for Plasmons}

In Chapter 5 we study the transmission of light propagating through hole-antennas and their near-field distributions. Using the experimental near-field distributions of hole-antennas with various hole diameters, we show that the NSOM signals contain both the in-plane electric and magnetic field components.

In Chapter 6 various phased antenna arrays, consisting of 2, 4, 5 and 10 individually controlled hole-antennas, are introduced. First, we show the controlled generation of SPPs by a single hole-antenna, which is illuminated with light with various degrees of polarization ellipticity. In the second part we investigate arrays of holes which are illuminated with various phase patterns, showing control on the SPPs' directionality and directivity. Finally we show the generation of localized patches of circular polarization, which are generated using a four hole-antenna system which generate two standing wave patterns of orthogonal polarization. A checkerboard pattern of circular polarized light with alternating handedness is formed and position controlled. 
Chapter 1 
Chapter 2

\section{Experimental techniques}

In this Chapter we describe the two main experimental setups used in this thesis. A dark-field spectroscopy system, capable of measuring one single optical antenna, and a near-field scanning optical microscope (NSOM) with amplitude, phase and polarization sensitivity are presented. In the last section of this Chapter a spatial light modulator (SLM) is incorporated into the NSOM, enabling control of the illumination amplitude, phase and polarization.

\subsection{Dark-field micro-spectroscopy}

Although optical antennas have been studied for more than a decade, and many basic antenna shapes have been explored, unanswered questions on how the shape of a single antenna and the coupling of multiple antennas affect their resonance behavior and emission patterns, still remain. In the previous Chapter, it was shown that the spectral response of an optical antenna is highly sensitive to changes in its geometry. This behavior will be explored further in Chapter 3, where we show that the resonance wavelength of an antenna can be shifted by hundreds of nanometers, by applying a small geometrical deformation. It is therefore of utmost importance to be able to correlate the changes in geometry with the change of resonance wavelength, which requires an experimental setup capable of measuring the spectral response of a single optical antenna.

An experimental strategy which is often applied, is to measure on a large matrix of antennas, in order to obtain a higher signal-to-noise ratio. However, this strategy puts a lot of pressure on the reproducibility of the structure fabrication. Although state of the art resolution was 
achieved in the fabrication of the structures in Chapters 3 and 4, small random geometrical variations in the order of 5-10 nm still remain. Taking an ensemble average of a large matrix of such structures would then wash out important spectral tuning effects.

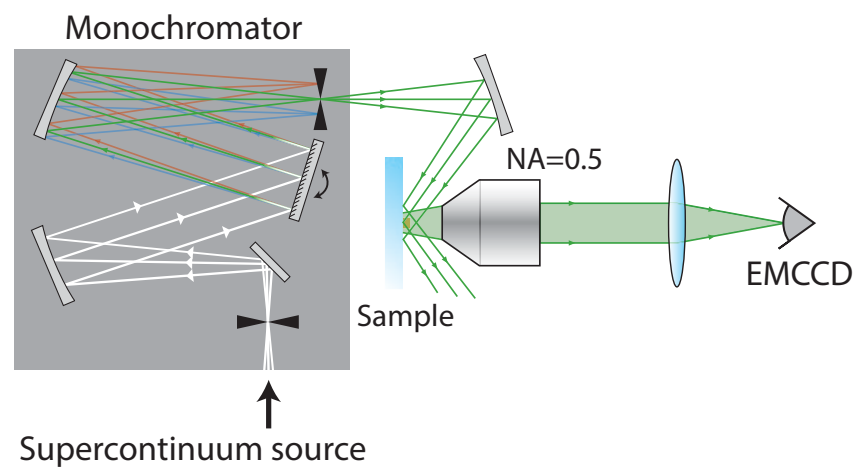

Figure 2.1: Dark-field spectroscopy setup. A schematic of the dark-field spectroscopy setup: a supercontinuum source is coupled into a monochromator after which a beam with a $2 \mathrm{~nm}$ spectral bandwidth is focused onto the sample under an angle of proximately $50^{\circ}$ with respect with the substrate. A 0.5 NA objective collects the light emitted from the nanostructures, after which it is focused on a cooled electron multiplying (EM) CCD.

When setting out to measure the spectral response of a sample, three different routes are possible: one could measure the amount of light absorbed by, or the amount of light scattered from the sample, or lastly one could investigate the absorption and scattering simultaneously and measure the extinction. When assuming uniform illumination, the absorptance $\left(A=\frac{\sigma_{a b s}}{S_{s p o t}}\right)$ can be found, which is the effective surface which has absorbed $\sigma_{a b s}$, also known as the absorption cross section compared to the surface of the illumination source $\left(S_{\text {spot }}\right)$. A similar procedure can be followed for the scattered light $\left(S=\frac{\sigma_{\text {scat }}}{S_{\text {spot }}}\right)$, with $\sigma_{\text {scat }}$ being the effective surface which has scattered light. Finally $T=1-(A+S)$ describes the difference of transmitted $(T)$, absorbed and scattered light.

The absorption approach was rejected because of the poor contrast one can obtain if one wants to measure a single optical antenna. In practice, measuring the light which is absorbed by a sample is not so easy. More often the extinction $\left(E=(A+S)=\frac{\sigma_{e x t}}{S_{\text {spot }}}\right)$ is measured by finding the ratio of light impinging on and transmitting through the sample. Because the extinction cross section of a single antenna is very small, in the order of $\sigma_{\text {ext }} \approx \lambda^{2}$, the surface area of the illumination spot needs 
to be of the same dimension in order to have a sufficient signal-to-noise ratio.

A dark-field microscope was built, which, by design, only measures the scattered light coming from the sample surface. This is done by illuminating the sample under an oblique angle; due to the angle of incidence neither the illumination nor the reflected light is collected by the objective (see Figure 2.1). Because only scattering is measured, the non-scattering substrate surface will not contribute to the overall signal, and only scattering particles will be detected. So instead of measuring a high background with a small signal (extinction experiments), a low background with a small signal is measured, which now enables us to use a cooled EMCCD camera and acquire with longer integration times without running the risk of saturating the camera.

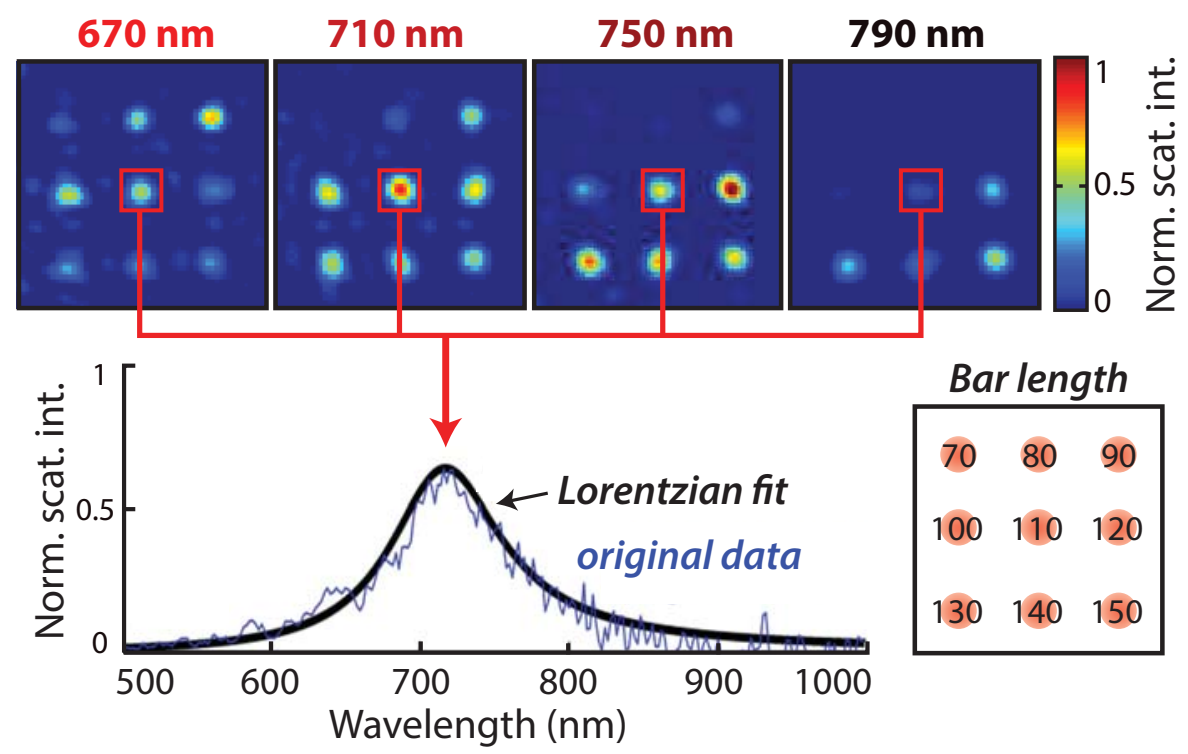

Figure 2.2: Dark-field micro-spectroscopy of bar antennas with different aspect ratios. (Above) Images of nine antennas with different lengths, varying from 70 to $150 \mathrm{~nm}$ with $10 \mathrm{~nm}$ increment, measured with four different illumination wavelengths. (Below) The scattering spectrum of a selected single bar antenna. Figure adapted from [68].

The dark-field spectroscopy setup uses the white light from a supercontinuum source (Fianium SC400-4) which is passed through a monochromator (Acton SP-2100i) to select the center wavelength of the illumination light with a $2 \mathrm{~nm}$ spectral bandwidth. The light is linearly polarized 
and focused weakly onto the sample under an angle of 50 degrees with respect to the substrate. While sweeping the monochromator output through the frequency range of interest, a series of images are collected on an EMCCD camera (Andor Ixion 878), using a 0.5 NA long working distance objective. We compensate for the chromatic shift in the focus of the collection objective by translating the sample stage during our measurements. This process is automated and before each measurement a calibration is made where the focus is determined at eight different wavelengths. A polynomial fit on the eight measurements is done, which is then used for positioning the sample stage during the measurement. Typically a maximum stage movement of approximately $15 \mu \mathrm{m}$ is needed for the objective which is used. At any given point in the field of view $( \pm 50 \times 50 \mu \mathrm{m})$, a spectrum is obtained by acquiring images at a range of different frequencies. The spectra are normalized by the spectral response of the system, which is obtained by measuring the scattering from a diffuser element.

Figure 2.2 shows four dark-field images, measured at different illumination wavelengths, of nine bar antennas having a length varying from 70 to $150 \mathrm{~nm}$ with $10 \mathrm{~nm}$ increments. The sample was illuminated with linearly polarized light which is orientated along the long axis of the bar antennas. The experiments were performed with an EMCCD camera which was cooled to $-60 C^{o}$, using a 0.2 second integration time. Nine spots can clearly be distinguished, showing a distribution of intensities which vary with increasing excitation wavelength, shifting towards antennas having larger aspect ratios. The scattering spectrum in Figure 2.2, is obtained by first subtracting the dark-current offset of each pixel and then calculating the mean value of the area indicated with a red square in Figure 2.2; this is done for the full wavelength range. The already well-known linear relation between resonance wavelength and aspect ratio, for antenna dimensions smaller than the wavelength [22, 49, 69, 70], is nicely demonstrated by our experiments on single bar antennas in Figure 2.3. Using the theory of Novotny on the effective wavelength scaling of optical antennas [49], it is possible to calculate the relation between the length of the rod antennas and their resonance wavelength for various rod diameters, indicated with the red area in Figure 2.3. The experimental results in Figure 2.3 which show a linear trend, highlight the capabilities of the dark-field spectroscopy setup, being able to measure individual optical antennas down to at least $70 \mathrm{~nm}$ in size. The linear trend also highlights the resolution and consistency of the fabrication techniques which were used. Because the linear trend of the bar 
length vs resonance wavelength lies well between the calculated trends of bars with a radius of 15 and $35 \mathrm{~nm}$, red area in Figure 2.3, we estimate that the dimension of the widths of fabricated structures have a standard deviation on the order of $5 \mathrm{~nm}$.

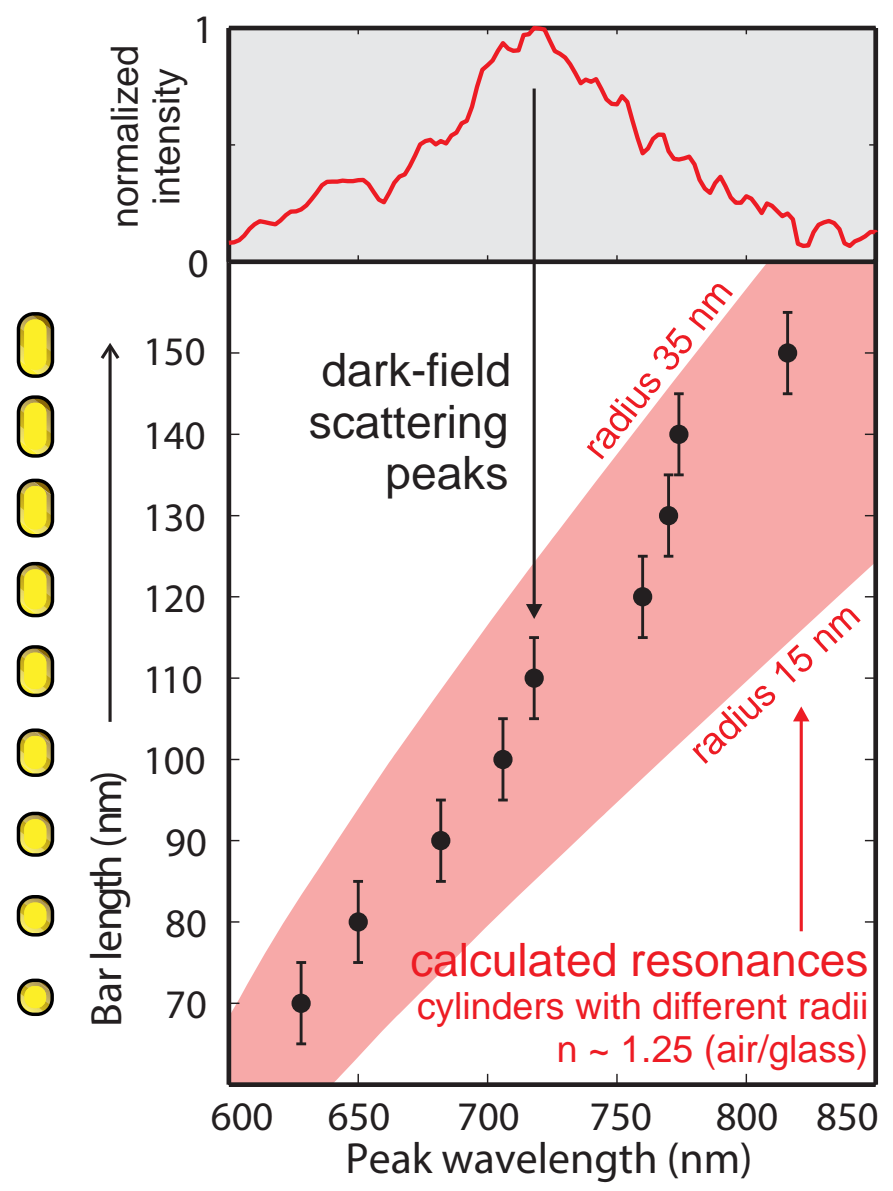

Figure 2.3: The effects of aspect ratio on the resonance wavelength. The resonance behavior of nine bar antennas with lengths varying from 70 to $150 \mathrm{~nm}$, are investigated by plotting their resonance wavelength as a function of antenna length. The resonance wavelength is extracted by fitting a Lorentzian function to the dark-field spectra (see above). The red area indicates the calculated resonances of cylinders with different radii, varying from $15-30 \mathrm{~nm}$, using the effective wavelength theorem [49]. Figure adapted from [68]. 


\section{$2.2 \quad$ Near-field microscopy}

In optics, resolution is defined as the minimum distance between two emitters such that both can still be distinguished as individuals. In 1881, Abbe described how the radius of a focused beam of light depends on the wavelength $(\lambda)$, the refractive index $(n)$ of the medium between the lens and the focus, and the angle $(\theta)$ with which the light is focused to a spot: $d=\frac{\lambda}{2 n \sin \theta}=\frac{\lambda}{2 N A}$ [71]. Although using a high numerical aperture (NA) objective, the focus can be on the order of $200 \mathrm{~nm}$, which is rather small compared to many optical systems like biological cells $(1-100 \mu m)$. This size limit is rather big compared to other optical systems like quantum dots and optical antennas $(10-400 \mathrm{~nm})$. Ever since the fundamental limit of resolution has been established by Abbe, there is an ongoing scientific interest in methods which could circumvent this fundamental limit.
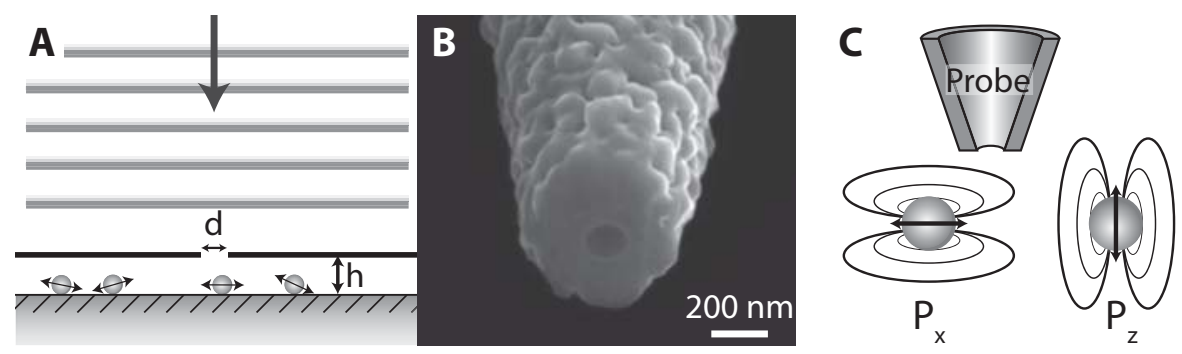

Figure 2.4: Aperture near-field probe. (A) a cartoon depicting the local excitation of dipole emitters by means of the evanescent field of a small hole, positioned close to the substrate surface (inspired by [21]). (B) shows a SEM micrograph of a near-field aperture probe used in our experiments. (C) a cartoon depicting the electric field distributions of a dipole oriented in the $x$ and $z$ direction, showing that only in-plane dipoles can be detected by the near-field probe (inspired by [72]).

In 1928, Synge proposed a revolutionary idea, which could theoretically beat the Abbe diffraction limit [73]. His idea was to position an opaque sheet with a small sub-wavelength hole, at a sub wavelength distance $(h<<\lambda)$ from the sample surface (see Figure 2.4 A). By illuminating the hole on one side, a small fraction of light could leak out on the other side in the form of an evanescent field. Because the evanescent field below the hole is highly localized, only a sub-wavelength volume underneath the hole is illuminated. By raster scanning the opaque sheet, while observing the light emitted from the sample surface, a sub-wavelength resolution image of the sample could be acquired. Due to the extreme 
experimental requirements on the positioning of the opaque sheet and the low intensity throughput as a result of using the sub-wavelength hole, it was not until 1984 that the first near-field images were recorded [74]. These first near-field images, reaching a resolution of $\lambda / 20$, were not taken by scanning an opaque sheet, but rather an aperture near-field probe, similar to one shown in Figure 2.4. The aperture probe, used in near-field measurements shown in this thesis, is made using the method described by Betzig et al. [75]. The sharp needle of the aperture probe is made by laser pulling an optical fiber, after which an optically thick aluminum coating is applied, finally a section of the tip is removed using a focused ion beam, to make an aperture.

Due to the ring-like shape of the aluminum coating of an aperture tip, only the in-plane fields are considered. The out-of-plane fields are generally disregarded as they are unable to generate a dipole moment at the NSOM aperture. Although for a different reason, if one sets out the measure dipoles in a solution or on a surface, only the dipoles which are orientated along the plane can be measured by an NSOM. The dipoles which are orientated towards the NSOM tip predominantly radiate inplane, away from the tip (see Figure $2.4 \mathrm{C}$ ).

The sample-probe distance is controlled using a similar method described by Karrai et al. [76], where they glued the near-field probe onto a piezoelectric tuning fork. The tuning fork is mechanically driven using a dither-piezo and the amplitude of the piezoelectric signal from the tuning fork is monitored. When the near-field probe is near the sample surface, the tuning fork resonance is slightly damped and shifted in frequency and the amplitude of the piezoelectric signal is reduced. A feedback mechanism is implemented in order to keep a constant amplitude, and hence a constant sample-probe distance, which is estimated to be approximately $20 \mathrm{~nm}$.

\subsubsection{Heterodyne detection}

The relative optical phase picked up by the near-field probe is detected by incorporating the near-field scanning microscope into a MachZehnder interferometer. In this interferometric detection scheme, which is also known as a heterodyne detection scheme, the signal from the near-field probe is combined with a frequency-shifted reference signal, also known as the local oscillator (see Figure 2.5). The frequency of the reference branch is shifted by $100 \mathrm{kHz}$ using two acoustic-optic modulators (AOMs). An AOM consists of a transparent dielectric material, in which a moving acoustical grating is generated with a very well defined 
frequency. The first AOM is used to frequency-shift the reference beam by $80.1 \mathrm{MHz}$ using the $(+1)$ grating order. By selecting the (-1) grating order of the second $\mathrm{AOM}$, which is driven at $80.0 \mathrm{MHz}$, a combined frequency shift of $100 \mathrm{kHz}$ is obtained. The angular frequency difference between the signal and reference branch thus becomes $\delta \omega=2 \pi \cdot 100 \mathrm{kHz}$.

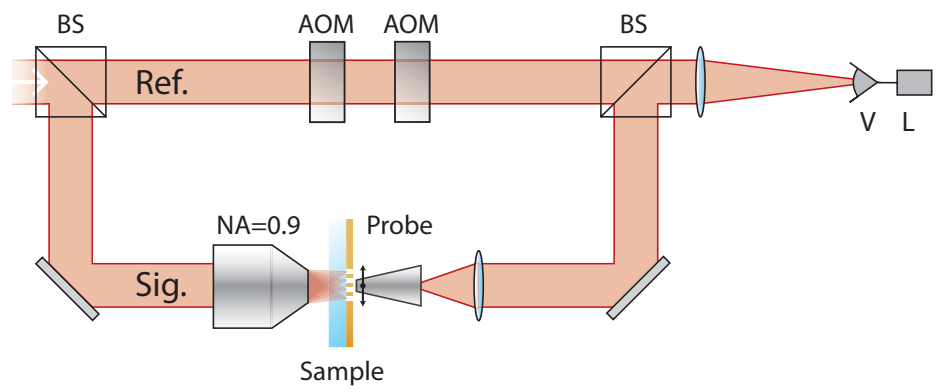

Figure 2.5: Phase-sensitive NSOM. Light from a continuous wave laser $(\lambda=$ $950 \mathrm{~nm}$ ) is split into a signal and reference branch. The light in the reference branch is frequency shifted by $100 \mathrm{kHz}$ using two AOMs. Light from the signal branch is focused on the sample using a 0.90 NA air objective, from where light is collected using a near-field probe. The light from the near-field probe is converted to free-space, whereafter the signal and reference branch are recombined using a beam splitter. The combined signals from the signal and reference branch impinging on the detector $(\mathbf{V})$ are analyzed by a lock-in amplifier (L).

The electric field of the signal $\left(\mathbf{E}_{S}\right)$ and reference branch $\left(\mathbf{E}_{R}\right)$ can be described as [77]:

$$
\mathbf{E}_{S}(x, y)=\mathbf{A}_{S}(x, y) \cdot \exp \left[i\left(\omega_{0} t+\phi_{S_{\perp, \|}}(x, y)+\beta_{S}\right)\right]
$$

and

$$
\mathbf{E}_{R}=\mathbf{A}_{R} \cdot \exp \left[i\left(\omega_{0} t+\delta \omega t+\beta_{R}\right)\right]
$$

were $\mathbf{A}_{S}(x, y)$ and $\mathbf{A}_{R}$ are the real amplitudes of the signal and the reference fields. The amplitude of the electric field detected by the fiber optic tip, depends on the position $(\mathrm{x}, \mathrm{y})$ where the light is picked up. The frequency of the laser source is described by $\omega_{0}$ and $\delta \omega$ is the $100 \mathrm{kHz}$ frequency shift which is induced by the AOMs. $\beta_{S}$ and $\beta_{R}$ are the phases acquired by the optical path length in the signal and reference branch, respectively, and can therefore contain contributions arising from environmentally induced drift. A distinction between the local phase distribution on the sample surface, described by $\phi_{S}(x, y)$, and large scale phase variation in $\left(\Delta \phi=\beta_{R}-\beta_{S}\right)$ is made. The experimental 
goal is to minimize the temporal variations of $\Delta \phi$, which can be done by reducing air flow, minimizing heat sources and reducing mechanical and acoustic vibrations near the interferometer. The voltage signal from the photodiode $\mathbf{V}$ can then be described in the following way:

$$
\mathbf{V}=\eta_{D}\left|\mathbf{E}_{R}+\mathbf{E}_{S}(x, y)\right|^{2}
$$

and

$$
\mathbf{V}=\eta_{D}\left\{\left|\mathbf{E}_{R}\right|^{2}+\left|\mathbf{E}_{S}(x, y)\right|^{2}+2 \operatorname{Re}\left[\mathbf{E}_{R}^{*} \mathbf{E}_{S}(x, y)\right]\right\}
$$

where $\eta_{D}$ is the detection efficiency of the detector. If the laser light is monochromatic and the amplitude $|E|$ is constant in time, equation 2.4 can be expanded to:

$$
\begin{aligned}
\mathbf{V} & =\eta_{D}\left\{\left|\mathbf{E}_{R}\right|^{2}+\left|\mathbf{E}_{S}(x, y)\right|^{2}\right. \\
& +2 \mathbf{E}_{R} \cdot \mathbf{E}_{S}(x, y) \cos \left[\delta \omega t+\phi_{S}(x, y)+\Delta \phi\right]
\end{aligned}
$$

The detected signal is sent to a lock-in amplifier (LIA), which is referenced to the $100 \mathrm{kHz}$ difference frequency. The lock-in amplifier selectively allows (and amplifies) signals in a narrow frequency band around $\delta \omega$, leaving only the $2 \mathbf{E}_{R} \cdot \mathbf{E}_{S}(x, y) \cos \left[\delta \omega t+\phi_{S}(x, y)+\Delta \phi\right]$ term of equation 2.5 to be considered. The frequency components of the signal, which lie within the narrow bandwidth of the LIA, are strongly amplified while the main contribution of the electronic noise, being white noise with a broad frequency distribution, is ignored. Because the LIA does not detect $\mathbf{E}_{S}(x, y)$ but the $2 \mathbf{E}_{R} \cdot \mathbf{E}_{S}(x, y)$ field contributions, the signalto-noise ratio is further enhanced by a factor of $\mathbf{E}_{R} / \mathbf{E}_{S}(x, y)$, which is also known as heterodyne gain. Generally the intensities in the reference and signal branches are in the order of $10^{-3}$ and $10^{-12}$ Watts, meaning that a heterodyne gain in the order of $\sqrt{10^{-3}} / \sqrt{10^{-12}} \approx 10^{4}$ can be achieved, which greatly improves the signal-to-noise ratio.

Now we will derive the complex signal (L). A lock-in amplifier requires a frequency reference $\left(\omega_{L}\right)$, which is used to generate an internal reference signal $\mathbf{V}_{L} \cos \left(\omega_{L} t+\phi_{\text {ref }}\right)$ with an adjustable phase offset $\left(\phi_{\text {ref }}\right)$. Only the last term of equation 2.5 is considered, as the first two terms will disappear in a later step, where the signal is put through a low pass filter. The experimental signal is multiplied by the internal reference signal, which for the two output channels $(\mathbf{X}, \mathbf{Y})$ has two phase offsets $\left(\phi_{\text {ref }}, \phi_{\text {ref }}+\pi / 2\right)$, giving: 


$$
\begin{aligned}
\mathbf{X} & =2 \mathbf{E}_{R} \mathbf{E}_{S}(x, y) \mathbf{V}_{L} \cos \left(\delta \omega t+\phi_{S}(x, y)+\Delta \phi\right) \cos \left(\omega_{L} t+\phi_{\text {ref }}\right) \\
& =\mathbf{E}_{R} \mathbf{E}_{S}(x, y) \mathbf{V}_{L} \cos \left(\left[\delta \omega-\omega_{L}\right] t+\left(\phi_{S}(x, y)+\Delta \phi\right)-\phi_{\text {ref }}\right) \\
& +\mathbf{E}_{R} \mathbf{E}_{S}(x, y) \mathbf{V}_{L} \cos \left(\left[\delta \omega+\omega_{L}\right] t+\left(\phi_{S}(x, y)+\Delta \phi\right)+\phi_{\text {ref }}\right) \\
\mathbf{Y} & =2 \mathbf{E}_{R} \mathbf{E}_{S}(x, y) \mathbf{V}_{L} \cos \left(\delta \omega t+\phi_{S}(x, y)+\Delta \phi\right) \cos \left(\omega_{L} t+\phi_{\text {ref }}+\frac{\pi}{2}\right) \\
& =\mathbf{E}_{R} \mathbf{E}_{S}(x, y) \mathbf{V}_{L} \cos \left(\left[\delta \omega-\omega_{L}\right] t+\left(\phi_{S}(x, y)+\Delta \phi\right)-\left(\phi_{\text {ref }}+\frac{\pi}{2}\right)\right) \\
& +\mathbf{E}_{R} \mathbf{E}_{S}(x, y) \mathbf{V}_{L} \cos \left(\left[\delta \omega+\omega_{L}\right] t+\left(\phi_{S}(x, y)+\Delta \phi\right)+\left(\phi_{r e f}+\frac{\pi}{2}\right)\right)
\end{aligned}
$$

Now a low pass filter is applied on $(\mathbf{X}, \mathbf{Y})$; when the reference frequency is set so that $\omega_{L}=\delta \omega$, only the difference frequency component $\left(\delta \omega-\omega_{L}\right)$ of equation 2.6 becomes a DC signal which is accepted through the low pass filter; (X,Y) become:

$$
\begin{aligned}
& \mathbf{X}=\eta_{D} \mathbf{E}_{R} \mathbf{E}_{S}(x, y) \mathbf{V}_{L} \cos \left(\left(\phi_{S}(x, y)+\Delta \phi\right)-\phi_{\text {ref }}\right) \\
& \mathbf{Y}=\eta_{D} \mathbf{E}_{R} \mathbf{E}_{S}(x, y) \mathbf{V}_{L} \cos \left(\left(\phi_{S}(x, y)+\Delta \phi\right)-\left(\phi_{\text {ref }}+\frac{\pi}{2}\right)\right)
\end{aligned}
$$

The complex signal $\mathbf{L}$ is obtained:

$$
\begin{aligned}
\mathbf{L} & =\mathbf{X}+i \mathbf{Y} \\
& =\eta_{D} \mathbf{E}_{R} \mathbf{E}_{S}(x, y) \mathbf{V}_{L} e^{i\left(\left(\phi_{S}(x, y)+\Delta \phi\right)-\phi_{r e f}\right)}
\end{aligned}
$$

Because the other contributions to the amplitude and phase in $\mathbf{L}$ are kept constant, any change in magnitude and phase in $\mathbf{L}$ is directly related to the amplitude $\mathbf{E}_{S}(x, y)$ and phase $\phi_{S}(x, y)$ distribution of the sample surface.

\subsubsection{Polarization resolved detection}

In order to simultaneously measure both orthogonal polarizations near a sample surface, the setup of Figure 2.5 is slightly altered (see Figure 2.6). A polarizing beam splitter cube is added in the detection scheme, and instead of one, now two photo diodes and lock-in amplifiers are used $[72,78]$. A half-wave plate $(\lambda / 2)$ is added to the reference branch, which is used to equally distribute the reference beam to both the detectors. In order to correctly map the orthogonal field components picked up by the NSOM probe onto the two detectors, a quarter-wave plate $(\lambda / 4)$ and half-wave plate are added to the signal branch. Like almost every fiber, the NSOM fiber suffers from birefringence, which is caused by strain due 


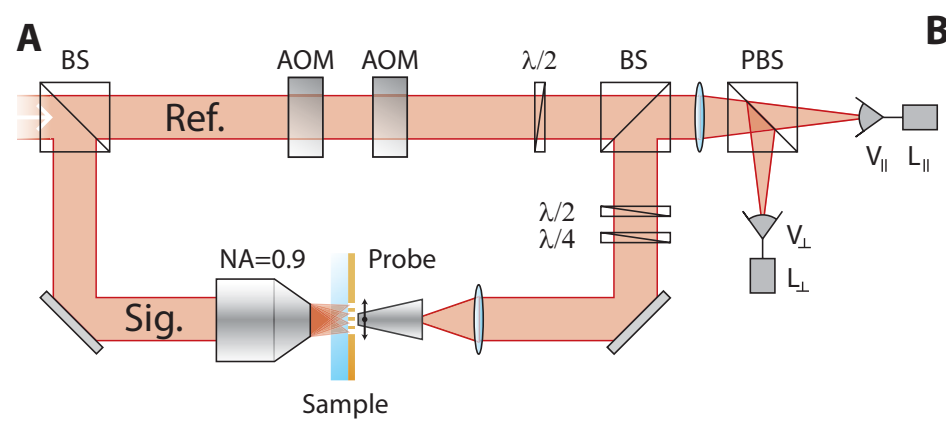

B Sample Pol.

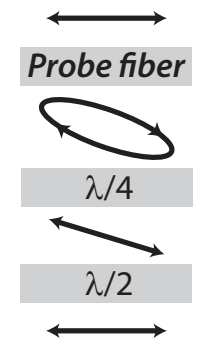

Detector

Figure 2.6: Phase and polarization sensitive NSOM. (A) the experimental setup shows great similarity to the one shown in Figure 2.5, with the key difference that a polarizing beam splitter cube is added in the detection scheme, in order to obtain polarization sensitivity. Two detectors and lock-in amplifiers are used to detect orthogonal polarization components $(\|, \perp)$. A half-wave plate is added to the reference branch which is used to distribute the reference light equally to both the detectors. In the signal branch, the light from the near-field probe is converted to free-space which is then passed through a $\lambda / 4$ and a $\lambda / 2$, whereafter the signal and reference branches are recombined using a beam splitter. In order to correctly map the orthogonal field components picked up by the near-field probe onto the two detectors $\left(V_{x}\right.$ and $\left.V_{y}\right)$, the angles of the $\lambda / 4$ and a $\lambda / 2$ in the signal branch are set accordingly, which in other words compensates for the birefringence of the fiber of the near-field probe (see B).

to bending or twisting of the fiber, whereupon the optical fiber itself will start acting as a $\lambda / 4$ and a $\lambda / 2$ plate. Consequently, linearly-polarized fields which were picked up by the NSOM probe, will become elliptically polarized when traveling through the NSOM fiber (see Figure 2.6 B). The angle of the $\lambda / 4$ plate in the signal arm is set such that it corrects any obtained elliptical polarization from the NSOM fiber. Then the $\lambda / 2$ plate in the signal arm is used to rotate the orthogonal axis of the linearly polarized light, so that the linear polarized components $(\|, \perp)$, which are picked up by the NSOM probe, contribute to $L_{\|}$and $L_{\perp}$. The birefringence of a fiber is stable as long as the fiber itself is not moved; the change in birefringence due to the scanning of the fiber tip over the sample surface, however, was found to be too small to measure. The position of the two wave plates in the signal arm are manually optimized each time a new NSOM probe is inserted. In the case of the gold surfaces with different hole geometries (Chapters 5 and 6), this is done by exciting the hole with a well-defined linear polarization and detecting the far-field signal at a distance of a couple of micrometers above the hole. Because the polarization state of light is not altered 
when propagating through a sub-wavelength hole, the correct positions for the $\lambda / 4$ and $\lambda / 2$ plates in the signal arm are found by maximizing the signal of one of the LIA's, and minimizing the signal on the other. This automatically also aligns the perpendicular polarization channel.

\subsubsection{Shaped illumination}

In this section, we describe the implementation of a spatial light modulator (SLM) into the polarization sensitive near-field microscope (see Figure 2.6), which enables full control of the optical state of the illumination of the sample (including amplitude, phase and polarization).

To fully characterize an optical system, one essentially sets out to find the transfer function $(T)$ of the system. The transfer function describes how the state of light passing through the system, is altered by the system. Using a well defined illumination source $(\Psi)$ and characterizing the optical response / state $\left(\Psi^{\star}\right)$, after the light has passed through the system, it is possible to find the transfer function of an optical system $\left(\Psi \cdot T=\Psi^{\star}\right)$, to the degree one is able to characterize $\left(\Psi^{\star}\right)$. However, as optical systems become more complex, the observed outcome highly depends on the optical state $(\Psi)$ of the illumination source. Because one can only experimentally change the illumination conditions to a certain degree, the transfer function is only partially known, and quite extensive a priory knowledge of the optical system is needed. If one could systematically vary the illumination conditions, while observing how this affects the response of the system, more elements of the transfer function could be explored and less a priori knowledge of the system would be needed. This approach has also been extensively used in the coherent control schemes which have been used for controlling the photo-physics of molecular systems [79-83]. The main difference between the coherent control scheme and the shaping of the illumination state for the NSOM, is that the first achieves control by means of temporal shaping, while the second achieves control by spatially shaping the illumination source.

Another reason for using a SLM to control the illumination state is that it enables the creation of otherwise not achievable illumination conditions. In the last couple of years, research of spatial control on surface plasmon polaritons has raised an increasing interest [84, 85], as SPPs show great potential for applications in sensing [86], photovoltaics [6], nanocircuitry $[18,87]$ and metamaterials $[58,88]$.

The spatial shaping of the illumination source, with full control of amplitude, phase and polarization, is achieved by incorporating a SLM 


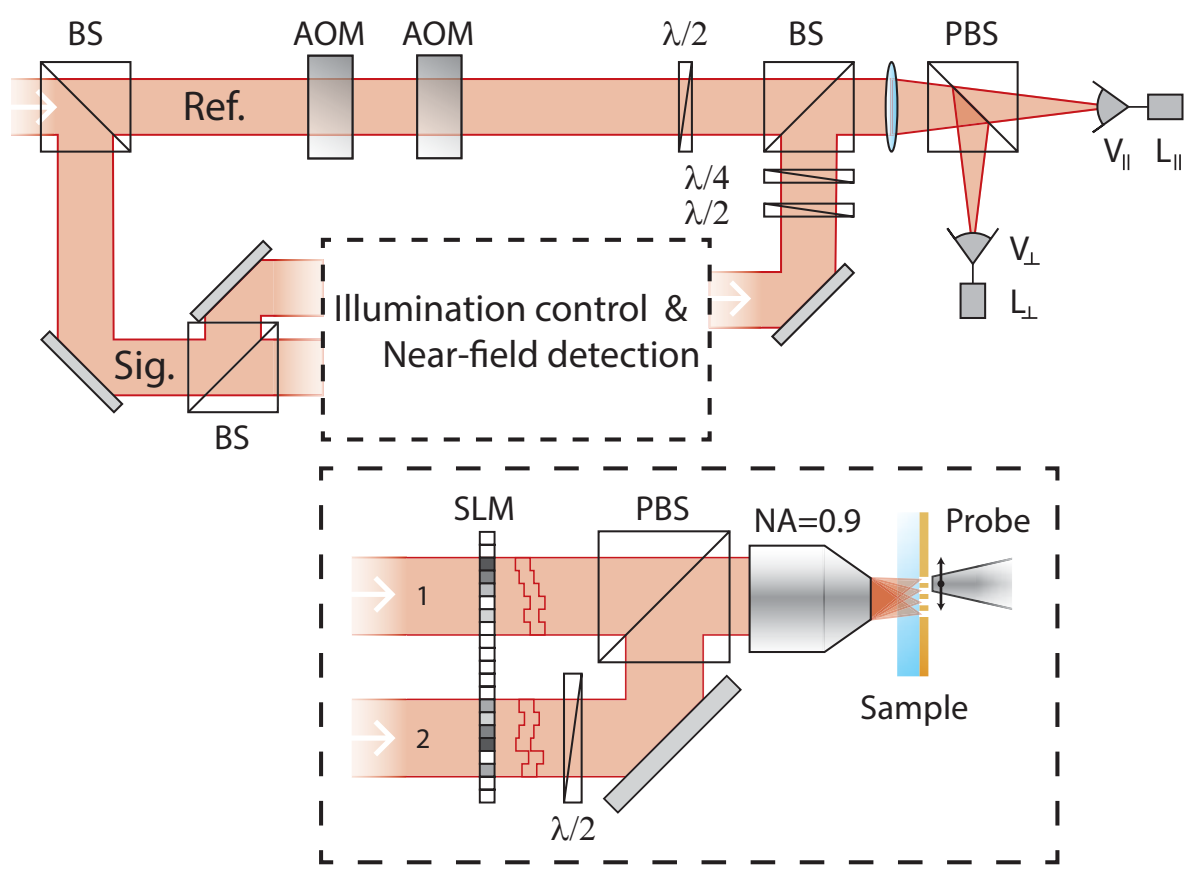

Figure 2.7: NSOM with spatial shaping. The experimental setup is similar to the polarization sensitive NSOM setup shown in Figure 2.6, with the addition of illumination shaping, located in the signal branch. A second Mach-Zehnder interferometer is created in the signal branch using a beam splitter cube. The phase front of the two resulting signal branches are shaped using a SLM, after which the second branch is passed through a half-wave plate $(\lambda / 2)$, which is set to rotate the polarization axis by $90^{\circ}$. The two beams are recombined using a polarizing beam splitter cube after which the light enters the objective.

(phase only shaping) into a second Mach-Zehnder interferometer in the signal branch (see Figure 2.7). For the sake of clarity the inset of Figure 2.7 shows a simplistic version of the optical shaping setup. Light in the signal branch is split into two branches using a beam splitter cube, and it is directed to the SLM (Pluto Holoeye (reflective)). The two shaped beams reflected from the SLM follow two separate paths in which a $4 \mathrm{f}$ system is incorporated, consisting of 2 lenses (see Figure 2.8). The $\left(0^{t h}\right)$ order reflection of the SLM is used, although this has the drawback of getting a $25 \%$ amplitude modulation when varying the phase of the pixels, which is related to the filling factor of the SLM surface. This effect can be reduced by imaging a grating on the SLM and using the $(1,-1)$ grating order. The light which is reflected from the unmodulated surface is reflected towards the $\left(0^{\text {th }}\right)$ order, and thus only shaped light is used in the experiment. However, using this method 
comes with the price of intensity loss, which is in the order of a factor of $2-5$. One of the two shaped beams is passed through a half-wave plate $(\lambda / 2)$, which is set to rotate the polarization axis by $90^{\circ}$, after which the two shaped beams are recombined using a polarizing beamsplitter cube. Both polarization axes can now be tuned separately. The last lens in the $4 \mathrm{f}$ system focuses the shaped beam on the back focal plane of the objective, consequently the phase shape which is applied by the SLM, is imaged on the back focal plane of the objective (see Figure 2.8).

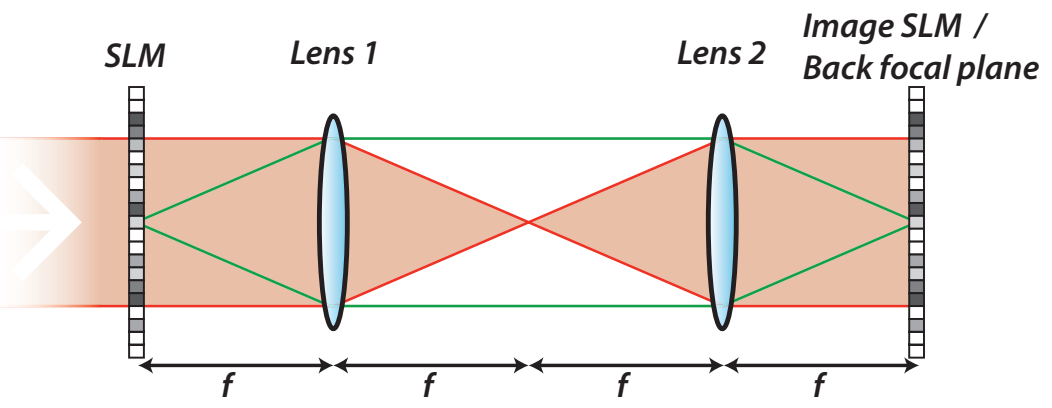

Figure 2.8: $4 f$ configuration. Cartoon depicting a $4 f$ configuration, the red surface depicts the respective beam waist, and the green line depicts the trajectory of light coming from the SLM and being re-imaged by the second lens.

Although the objective contains multiple optical elements, it can still be described as a simple lens; light passing through the back aperture of the objective is 2D Fourier transformed. Figure 2.9 shows three examples which visualize how the shape of the phase-front affects the shape of light which is focused by a lens. In all three cases the amplitude distributions are kept equal, and show a Gaussian shape. In the first example a flat phase-front is applied, resulting in a miniature Gaussian beam profile at the focus of the lens. The second example shows a phase-front which is tilted in the horizontal axis, and again a miniature Gaussian beam profile is observed, however this time the focus is shifted to the right. This example is similar to the situation where light passes through a lens under an oblique angle, which is recreated by the SLM by applying a phase slope. Increasing the periodicity of the phase slope effectively increases the angle of incidence on the lens. The last example shows the typical SLM configuration which is used in our experiments, where in this case four foci are generated. The positions of the foci are determined by applying different horizontal and vertical phase slopes for every mode. The area of the phase-front which is associated with each focus, is indicated with the red dashed lines in the middle panel. The 
foci on the right panel, correspond to the four phase shapes indicated with the numbered circles. In the experiments, the main focus spot is intentionally displaced from the area of interest. By doing so, control of the amplitude $(A)$ of each focus is possible. This is done by changing the area of the mode on which the phase slope is applied. How the amplitudes of the foci are affected by the different filling-factors is shown in Figure 2.9, where the amplitudes of the foci going from 1 to 4 show a strong reduction. The phases of the foci can simply be varied by applying a phase offset $(\phi)$ on the individual phase fronts of the four modes. Because the amplitudes and phases of the four modes are shaped separately for the two polarization axes (see Figure 2.7), any arbitrary polarization state can be made. The effects of a half-wave plate can be mimicked by changing the amplitude ratio of the two polarization axes $\left(\theta_{\lambda / 2}=\tan \left(A_{\perp} / A_{\|}\right)\right)$. A quarter-wave plate can be mimicked by changing the overall phase difference between the two polarization axes $\left.\left(\theta_{\lambda / 4}=\phi_{\perp}-\phi_{\|}\right)\right)$, where the combined fields of both polarization axes show a linear polarization if the two axes are in-phase, and a circular polarization if the axes show a phase difference of $\pi / 2$. Even the handedness of the circularly polarized light can be selected by choosing a positive or a negative phase difference of $\pi / 2$. 

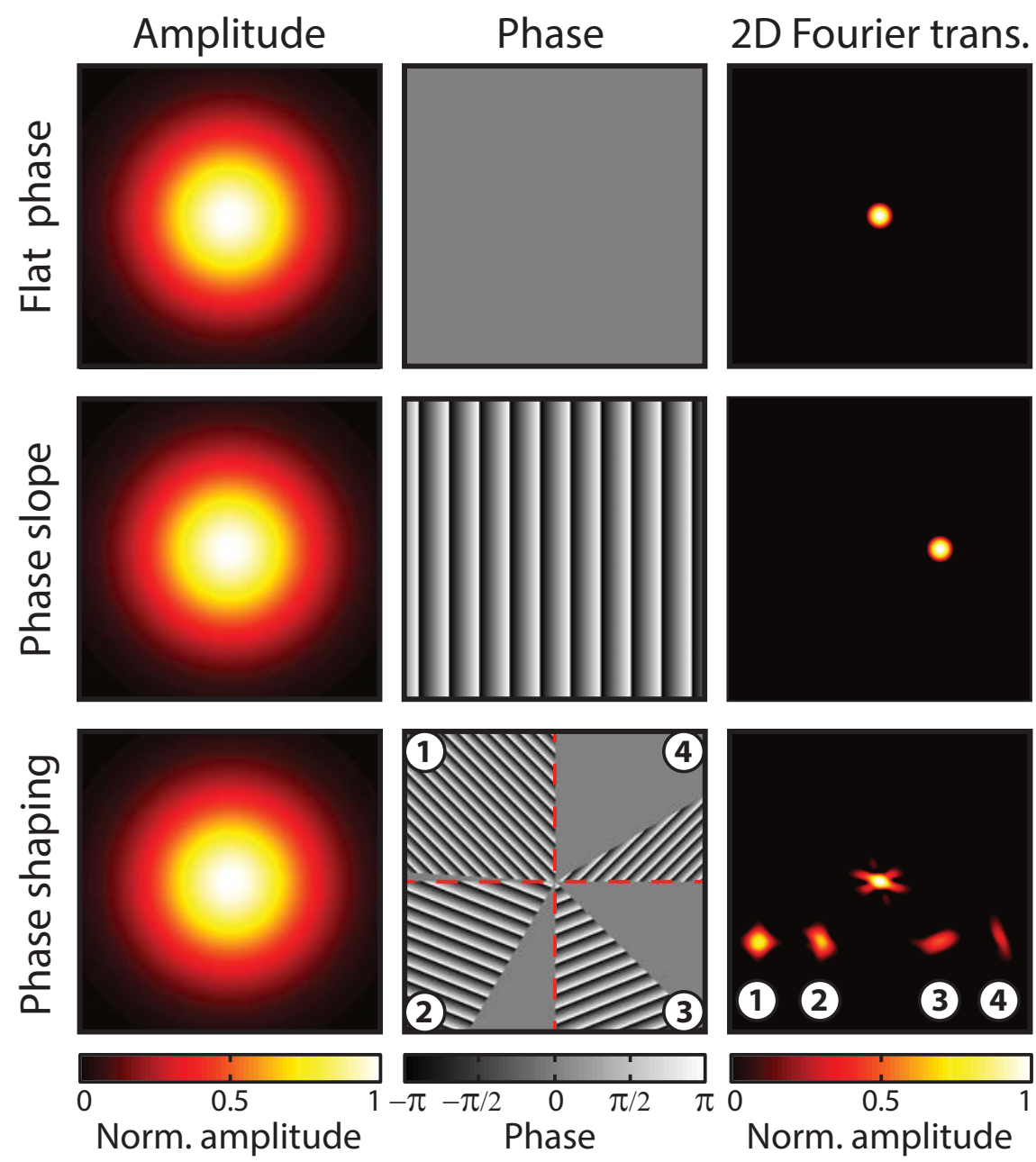

Figure 2.9: Phase shaping. Three numerical examples which visualize how the shape of the phase-front affects the shape of light, which is focused by a lens (equal to a 2D Fourier transform). In all three examples the starting amplitude shapes are similar and show a Gaussian shape. The lower three images show the phase shaping of four modes, indicated with the numbered circles. The amplitudes of the four foci are regulated by the respective filling factor of the phase slopes, which are associated with the four modes, indicated with red dashed lines in the central image of the lowest row of images. 


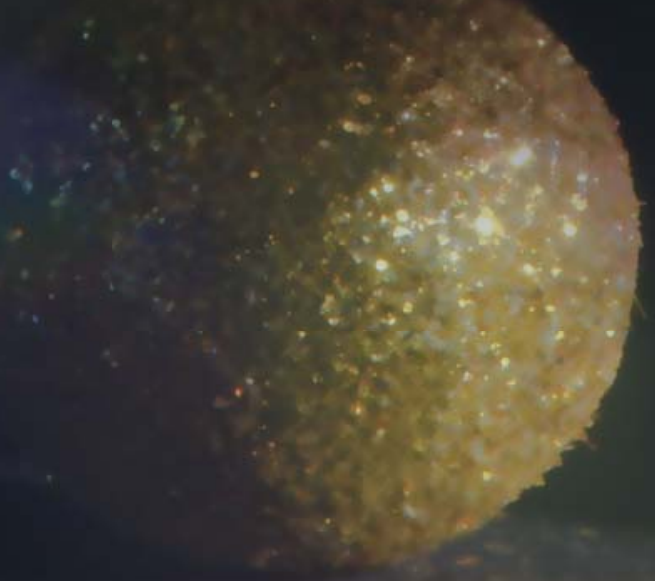




\section{Part I}

Antennas for Light 



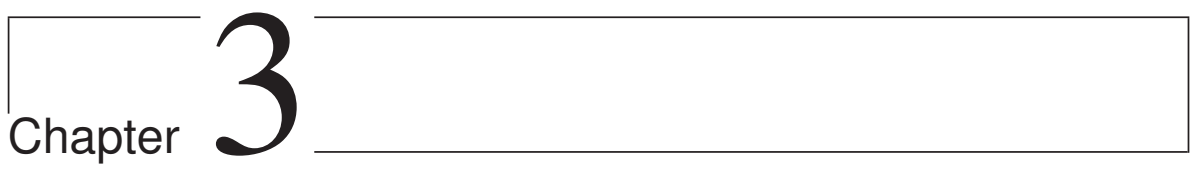

\section{Width modulated antennas}

Small metal structures sustaining plasmon resonances in the optical regime are of great interest, due to their large scattering cross sections and ability to concentrate light. In this Chapter, we study the dipolar plasmon resonances of optical antennas with a constant volume and a sinusoidal modulation in width. We observe experimentally that by changing the relative phase of the modulation, with a small $10 \mathrm{~nm}$ modulation amplitude, the resonance shifts over $160 \mathrm{~nm}$. Using simulations we show how this simple design rule can create resonance shifts greater than $600 \mathrm{~nm}$. Furthermore we investigate the magnitude of the near-field outside of the structures, and show that for conserved volume particles near-field and spectrum cannot be independently tuned. We discuss the applicability of several often used engineering models, which relate certain aspects of the antenna shape to their resonance wavelength. The antenna design we tested combines a large spectral tunability with a small footprint: all the antenna dimensions are factor 7 to 13 smaller than the wavelength, and hold potential as a design element in meta-surfaces for beam shaping.

\subsection{Introduction}

Optical antennas are small, wavelength-scale metal elements that convert light from the far-field into localized energy in the near-field, or vice versa $[1,20,48]$. As such, they can be used, for instance to modify the emission and absorption of quantum emitters [89], increase the response of refractive index based sensing [90,91], or enhance non-linear optical effects [92]. They are also very effective, polarization sensitive, scatterers for light. This property for instance enables the manipulation of the phase front of propagating light to obtain tailored optical beams [32,93], or it can be used to enhance absorption in photovoltaic devices [6]. 
In almost every application of optical antennas, control over the spectrum is critical. In addition to this, many applications require near-field electric field enhancement. For that reason, a large effort has been put in finding handles to control these two properties, using the geometric shape of the antenna [94,95]. Unfortunately, only for a few simple geometries are analytic expressions available, namely for ellipsoids within the quasi-static approximation [37,38], with extensions based on this to describe for instance cubic shapes [41]. Semi-analytical descriptions, such as the effective wavelength scaling [49], are successful in describing the spectral properties. Despite the fact that we restrict ourselves here to the simplest class of antennas that exhibit dipolar resonances, and are considerably smaller than the wavelength of light, such that the problem effectively relies on solving an electrostatic problem, there is a clear lack of engineering rules. Because of this, the vast majority of studies relies on brute-force numerical techniques such as FDTD, BEM or FEM to calculate near-fields and spectra. Luckily, these are nowadays fast enough even to enable computer based optimization strategies [96, 97]. These numerical methods, however, deliver limited physical insight. The lack of engineering design rules, to relate shape with spectrum and nearfield, clearly merits the further exploration of the influence of shape on the antenna properties.

In this Chapter we study both experimentally and theoretically the tuneability of the dipolar resonance frequencies of optical antennas, that are essentially a rectangular box with a sinusoidal modulation of width, as illustrated in figure Figure 3.1. The volume and footprint - defined as the cross sectional area and distribution of widths, respectively, remain constant. The only variations between the structures are (1) the phase of the cosine with respect to start and end of the particle and (2) the amplitude of the sinusoidal modulation. The structures show a smooth transition from convex to concave with decreasing phase value. For more information on how these structures where fabricated see Appendix A.3. The experimental results we obtain, portray nicely how small changes in the shape lead to changes in resonance wavelength, spanning $160 \mathrm{~nm}$ from $660 \mathrm{~nm}$ to $830 \mathrm{~nm}$, while keeping the antenna compact in size, with both width and height below $100 \mathrm{~nm}$. We discuss the applicability of several conventional models for the observed spectral shifts, and finally show both in experiment and theory what happens if asymmetry is introduced in these structures. 


\section{Different geometries}

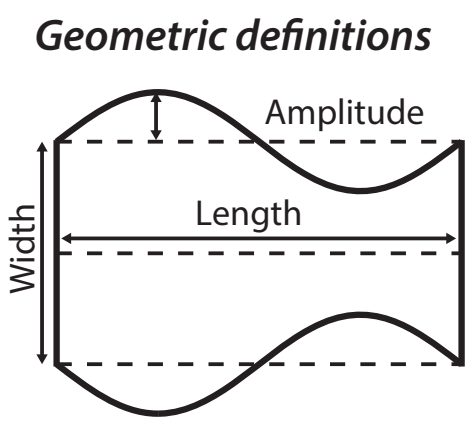

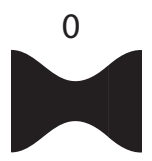

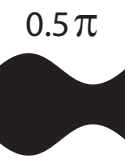

$\pi$

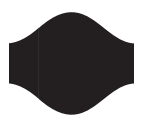

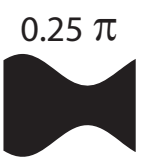

$0.75 \pi$

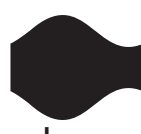

bar

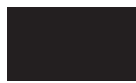

Fabricated structures
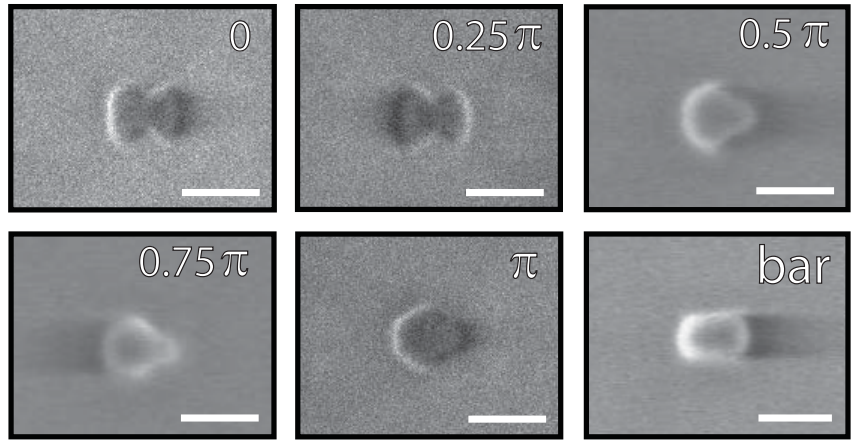

Figure 3.1: Illustrations of the studied structure. (Top left) a graphical depiction of the investigated structure, with the different geometrical definitions used in this Chapter. (Top right) the different geometries studied in this Chapter, which are obtained by changing the phase value of the sinusoidal modulation. (Below) SEM micrographs of an unmodulated bar and 5 width modulated nanoantennas, having different respective modulation phase values and an amplitude of $10 \mathrm{~nm}$. The scale bar indicates a length of $100 \mathrm{~nm}$.

\subsection{Modulation phase}

In Figure 3.2 the effect of the phase value of the sinusoidal modulation on the resonance wavelength of an optical antenna is depicted. It shows experimental scattering spectra of an unmodulated bar antenna (in blue) having a resonance wavelength at $740 \mathrm{~nm}$, and five modulated antennas with modulation phases varying between 0 and $\pi$ having a modulation amplitude of $10 \mathrm{~nm}$. The bar antenna has the following dimensions: 100 $\pm 10 \mathrm{~nm}$ long, $70 \pm 10 \mathrm{~nm}$ wide and $35 \pm 10 \mathrm{~nm}$ high. The experimental scattering spectra of individual antennas are obtained using the dark- 
field micro-spectroscopy setup, which is described in Section 2.1. The excitation source is linearly polarized and orientated along the longitudinal axis of the antennas. A single resonance peak is observed for each of the modulated antennas, where for a phase factor changing from 0 to $\pi$, a monotonic decrease in resonance wavelength is observed from 830 to 670 $\mathrm{nm}$. The total range of resonance wavelengths that can be achieved with
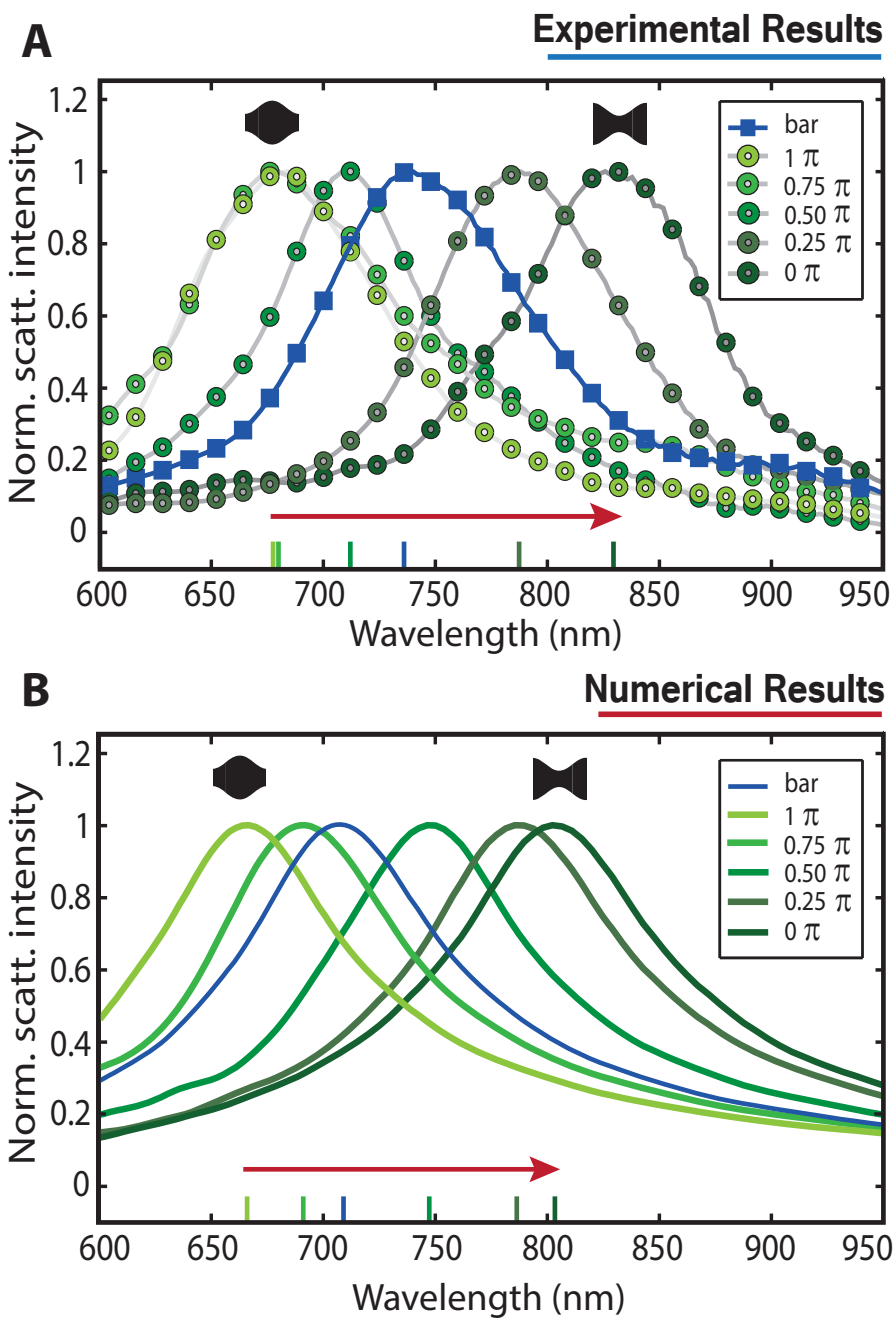

Figure 3.2: Scattering spectra of width modulated antennas with various modulation phases. (A) experimental data, (B) numerical calculations showing scattering spectra of width modulated antennas, having different respective modulation phase shifts and a modulation amplitude of $10 \mathrm{~nm}$. The scattering spectra are normalized to their respective maximum scattering intensity. 
this collection of structures is approximately $160 \mathrm{~nm}$. Good agreement is found between experiments and numerical calculations (Figure 3.2 B), though experiments show a $20 \mathrm{~nm}$ larger red shift and a $10 \mathrm{~nm}$ smaller blue shift. These small differences are attributed to small fabrication imperfections. The numerical calculations of the scattering intensity were performed using a finite element method technique, the details of the method which is used to obtain the scattering cross intensity spectra are described in Appendix B.
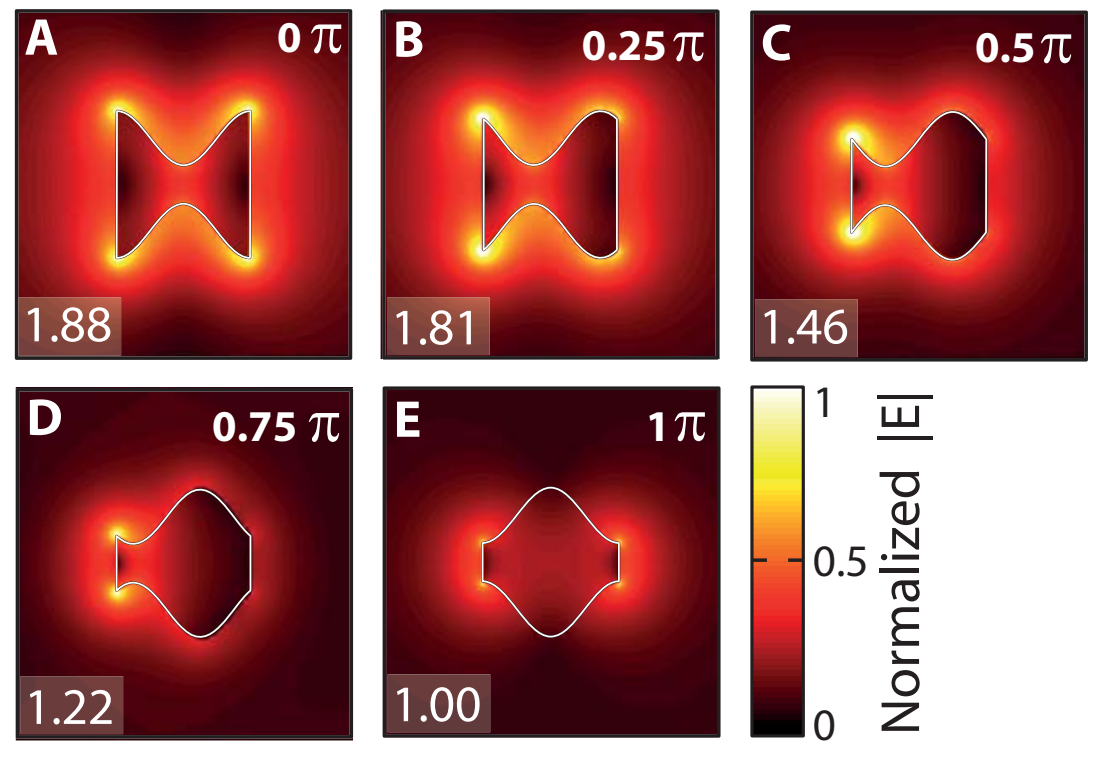

Figure 3.3: Numerical calculations of the field distributions of various width modulated antennas. (A-E) numerically calculated absolute electric field distributions of five modulated antennas, with different modulation phases and a modulation amplitude of $20 \mathrm{~nm}$. The electric field distributions have been normalized to the maximum value of $\mathrm{E}$. A ratio of the overall cumulative electric field distribution outside the metal, is shown in the bottom left corner of each respective image.

Figure 3.3 (A-E), shows numerical calculations of the electric field distribution of width modulated antennas, with different modulation phases and a modulation amplitude of $20 \mathrm{~nm}$. The electric fields are calculated using a finite element method, for which the details are described in Appendix B. The electric field distributions are all in the same color scale, allowing comparison between the figures, but they are all obtained at their respective resonance frequency. In the lower left corner of each figure the value of the average electric field amplitude outside the metal, 
inside a 225 by $225 \mathrm{~nm}$ area, is shown as an indication of the relative near-field enhancements of the different geometries. It can be seen that the concave structure with phase value 0 , has 1.9 times higher near-field than the convex structure with phase value $\pi$, with the other values located in between, following a monotonic decrease with phase value. Interestingly, the concave structures show a field distribution which is localized more towards the concave center, in comparison to the convex structures, where the fields are more localized towards the end-facets of the antenna. Also, with increasing phase values, the field distributions become asymmetric, in the sense that the left half of the antenna shows a higher field concentration compared to the right half. In the case of a $0.75 \pi$ modulation phase, the left half of the antenna is almost 2 times stronger compared to the right half.

\subsection{Modulation amplitude}

The relation between the observed resonance red-shifts and the geometrical shape of the particle is further investigated numerically. Figure 3.4 (A) shows the resonance wavelength as a function of modulation amplitude, varying from $0 \mathrm{~nm}$, which is equal to an unmodulated bar antenna, to $30 \mathrm{~nm}$, in which case the maximum and minimum width of the antenna are 130 and $10 \mathrm{~nm}$, respectively. The resonance wavelengths calculated are extracted from numerically calculated scattering spectra. By changing the amplitude in the sinusoidal modulation, the resonance wavelength can be tuned over a spectral range of $600 \mathrm{~nm}$. This is a large effect and in order to obtain a similar resonance shift in the ordinary bar antenna, one would have to increase its length by a factor of 2.8, from 100 to $280 \mathrm{~nm}$. Figure 3.4 (A) shows a similar trend as seen in Figure 3.2, in which the resonance wavelength of the modulated antenna is redshifting for a decreasing modulation phase from $\pi$ to 0 . However, by incorporating the modulation amplitude, it becomes clear that the effect resulting in a blue shift is relatively small in comparison to the effect leading to a redshift. This becomes even more evident in the case of an antenna with a modulation phase of $0.75 \pi$, which experiences a blue shift for modulation amplitudes up to $15 \mathrm{~nm}$, and a larger red shift at larger modulation amplitudes. Interestingly, the resonance wavelength at a modulation amplitude of $17.5 \mathrm{~nm}$ is exactly the same as an unmodulated bar antenna. It therefore seems that two competing processes are influencing the resonance wavelength. 

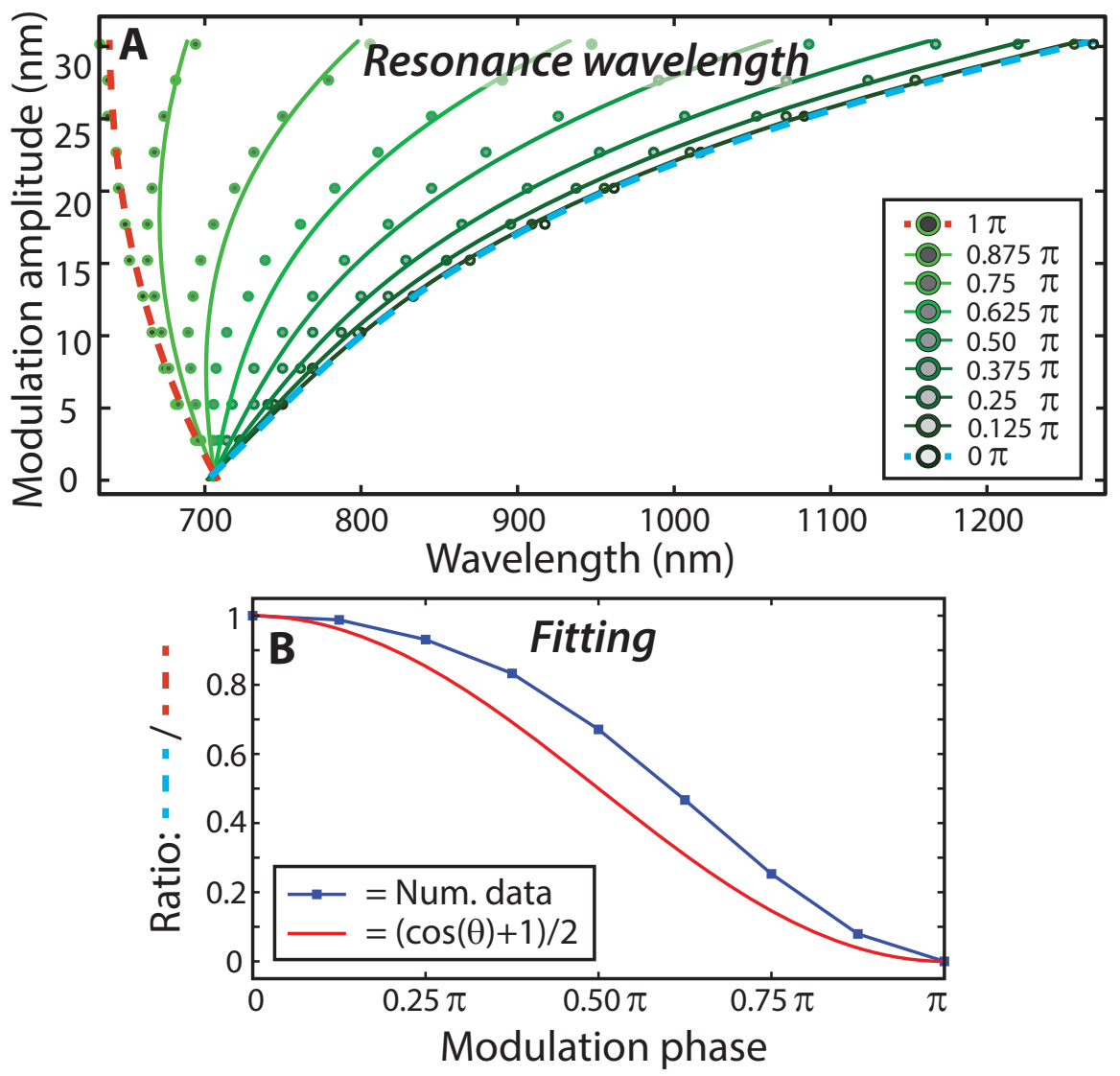

Figure 3.4: The effects of modulation amplitude and phase on resonance wavelength. (A) numerical calculations of various width modulated antennas and their respective resonance wavelength. The resonance wavelength of the nine different types of modulated antennas, having different modulation phases, are calculated for various modulation amplitudes. The two most extreme types of modulated antennas with a modulation phase of 0 (blue dashed) and $\pi$ (red dashed) are fitted with third order polynomial function. After which the ratio of these two functions is used to fit the other seven modulated antenna types (indicated with the solid lines). (B) the ratio of the polynomial functions, which describe 0 (blue dashed) and $\pi$ (red dashed), respectively, are used to describe the intermediate modulated antennas with varying modulation phases (blue solid). 
Because the modulated antennas show a smooth transition between a concave and a convex shape, we now try to use the modulation amplitude trends of the two most extreme shapes to describe the resonance behavior of the intermediate shapes. By doing so we try to extract a general relation between the shape of the modulated antennas, and their resonance behavior. First, the two most extreme types of modulated antennas with a modulation phase of 0 (blue dashed) and $\pi$ (red dashed) are fitted with third order polynomial function $\left(f_{0}(\lambda), f_{\lambda}(\lambda)\right)$, see Figure $3.4(\mathrm{~A})$. After which the ratio of these two functions is used to fit the other seven modulated antenna types (indicated with the solid lines in (A) and a blue line in (B)), using the following fitting function:

$$
f_{\phi}(\lambda)=R_{0, \pi} \cdot f_{0}(\lambda)+\left(1-R_{0, \pi}\right) \cdot f_{\pi}(\lambda)
$$

where $R_{0, \pi}$ is the fitting ratio of the two polynomial fits $f_{0}(\lambda)$ and $f_{\pi}(\lambda)$, which describe the modulation amplitude curves of the structures with a modulation phase of 0 and $\pi$, respectively. Only a single fitting parameter is used $\left(R_{0, \pi}\right)$, implicitly assuming that the intermediate structures are described as a linear combination of the two extreme antenna shapes. This fitting approach is not based on any theoretical model, but rather aims to visualize a possible relation between the resonance behavior, and the shape of the two structures having the extreme modulation phases. We find that this simple fitting approach works very well, illustrated by the solid lines in Figure 3.4 (A), which follow the general trend of the numerical data points. The fact that the resonance behavior of the intermediate modulated structures can be expressed by the resonance behavior of the two extremes, even for varying modulations amplitudes, is not self-evident. The fact that this approach works at all suggests that there is a clear relation between the shape of the modulated structures and their resonance wavelength. In (B) we now plot $R_{0, \pi}$ for the different modulation structures, or in other words as a function of modulation phase. We observe that for structures having a modulation phase which lies close to 0 or $\pi$, the slope of $R_{0, \pi}$ is almost flat, whereas the largest slope is found at $0.5 \pi$, showing similarities to a $(\cos (\theta)+1) / 2$ function, plotted in red. This function can be related to the ratio with which the end cap length changes as a function of modulation phase, for antennas having a modulation amplitude of $35 \mathrm{~nm}$. Although this find is intriguing, no general relation between the resonance behavior of the various antenna shapes, and the cosine function has been found. 


\subsection{How to model the relation between shape and resonance?}

Although several theories have shown to be able to relate the field distribution of an optical antenna and its resonance behavior, there is still a great need for design rules, as only in specific cases have relations between shape and resonance been found. For every new structure it is therefore still necessary to perform rigorous calculations to extract the resonance behavior. It would be beneficial to obtain more insight in the reason for the observed spectral shifts of the various structure shapes. Below we discuss the applicability and the predictive power of four commonly used models, which are all based on the assumption that the resonant behavior of an optical antenna can be described in the electrostatic regime.

\subsubsection{Fredholm integral}

In an earlier study on concave and convex shaped optical antennas [94], the resonance behavior of the two different antenna shapes was understood by means of a surface integral eigenvalue based problem. The different resonance behavior of concave and convex antennas is related to the combination of their geometrical shape and charge distribution. The geometry dependent eigensolution for the surface charge density and the eigenvalues, which describe the resonance frequency of the antenna, are found using the Fredholm integral equation [95], which in this case is shown in its two dimensional form:

$$
\sigma(Q)=\frac{\epsilon(\omega)-1}{\epsilon(\omega)+1} \int_{S} \sigma(M) \frac{\vec{r}_{M Q} \cdot \hat{n}_{Q}}{\pi\left|\vec{r}_{M Q}\right|^{2}} \cdot d S_{M}
$$

where $\sigma(Q)$ is a surface charge density at point $\mathrm{Q}$, and $\epsilon(\omega)$ is the antennas' dielectric constant. The integration is performed on the surface of the antenna, where $\vec{r}_{M Q}$ describes the vector which connects any point $(\mathrm{M})$, on the antenna surface with a point of interaction $(\mathrm{Q})$ and $\hat{n}_{Q}$ is the normal to the surface at the point $Q$, see Figure 3.5. In order to obtain the exact resonance frequency of an antenna a full numerical calculation, solving the eigen-value problem given by the Fredholm surface integral, which will provide the correct surface charge distribution and resonance frequency. A conceptual explanation is provided in [94], where it is pointed out that for concave particles, the normal to the surface can point in a counter direction to the charge separation vector, which thus results in a local negative value of the scalar product within the integrand of Equation 3.2, indicated with situation 1 in Figure 3.5. It is then argued 


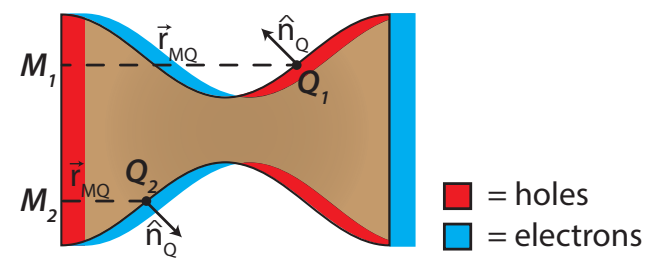

Figure 3.5: Fredholm integral method. Cartoon depicting the Fredholm integral method applied on two positions on the surface of a concave antenna. The red and blue areas indicate the accumulation and depletion of charge, due to the oscillatory movement of the free electrons.

that because of these negative values in the surface integral, which occur in the concave and not in the convex antennas, a resonance red-shift is expected. Although the method of acquiring the resonance behavior of an optical antenna, described in [95], has proven to be generally valid, the conceptual explanation provided in [94] is an oversimplification, and actually leads to a blueshift instead of a redshift. This is exemplified in Figure 3.5, where in the second situation two points on the antenna surface are investigated, which lie closer together. In this case, the scalar product in the integrand will lead to a positive contribution, which actually is of a larger magnitude than situation one, as $\vec{r}_{M Q}$ in this case is smaller, and using the same line of thought would lead to a blueshift. Although many methods are capable of extracting the resonance behavior of optical antennas when the field distribution is known, they often lack predictive power, making it seemingly impossible to describe the resonance behavior based on the antenna shape alone.

\subsubsection{Oscillatory movement of an electron cloud}

Plasmonic resonances in optical antennas have been described as the oscillatory movement of a free electron cloud on top of a rigid, uniform background charge, representing the ion cores of the metallic antenna [48]. The movement of the electron cloud in turn is often simplified to a simple mass-spring resonator. The mass-spring model has often been a successful analogy to obtain simple physical insight into the behavior of optical antennas [48]. In this model, the resonance frequency $\omega_{\text {res }}=\sqrt{k / m}$ is determined by a spring constant $k$ and a mass $m$, which are the elastic constant of the restoring force and the total effective mass of the whole antenna, respectively. Both the spring constant and the mass are determined by geometrical factors. While the mass is represented as simply scaling with the mass of the electrons and thus scaling with volume, the relation between the geometry and the 
spring constant is not obvious.

Similarly, the resonance frequency of relatively simple structures have been analytically derived by modeling the conduction electrons as an incompressible and irrotational charge fluid [98], and expressing the movement of the free electrons in terms of their potential (V) and kinetic energy $(\mathrm{T})$, and solving the Lagrangian. Although the method of deriving the correct field distributions and currents for the antenna shapes is based on a fluid approach, the same model is used to describe the resonance of the antenna in terms of the movement of the free electrons.
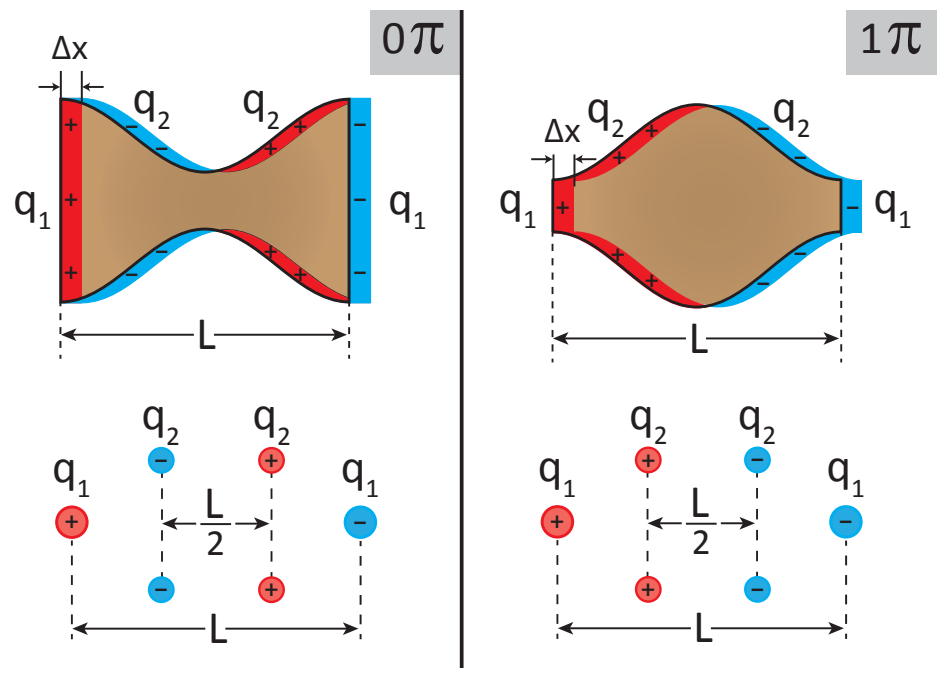

Figure 3.6: First order approximation of the charge distribution for the most extreme antenna shapes. Cartoons, depicting the first order approximation of the charge distribution, if the respective electron cloud would be shifted by $\Delta x$, shown for the two optical antennas which demonstrate the most extreme resonance shifts.

Similarly, an intuitive model which describes the linear relation between the aspect ratio of a bar antenna and its resonance wavelength, was proposed by Hecht $[23,48]$, where the work involved in displacing the electron cloud from the ionic backbone is calculated. In this model, the dipolar charge distribution on either side of the antenna end-caps is described as two point charges, which are displaced by a distance equal to the length of the antenna. A clear relation between the aspect ratio and the resonance wavelength of the antenna was shown. However, this model implicitly assumes a dipolar charge distribution, which for more complex mode profiles such as the concave antennas is not the case, see 
Figure 3.6. The work needed in the displacement of the electron cloud is calculated by:

$$
U_{E}=\frac{1}{2} \sum_{i=1}^{N} q_{i} \sum_{j=1}^{N(j \neq i)} \frac{1}{4 \pi \epsilon} \frac{q_{j}}{r_{i j}}
$$

where $U_{E}$ is the electric potential energy, $q_{i, j}$ are the various point charges located on the antenna surface, and $r_{i j}$ depicts the distance between $q_{i, j}$. Using this description it becomes clear, that for the concave structures where the distance between the charges with opposite signs is smaller, the potential energy increases. As such, this model does not deliver sensible results and leads to resonance blueshifts instead of redshifts.

\subsubsection{Effective wavelength scaling}

Because at optical frequencies metals start to act as strongly coupled plasmas, most relations described in conventional antenna theory do not hold anymore. However, Novotny has shown that the resonance wavelength $(\lambda)$ of thin nanowire antennas can be described by conventional antenna theory, by replacing $\lambda$ with a linearly scaled effective wavelength $\lambda_{\text {eff }}=n_{1}+n_{2} \lambda / \lambda_{p}$, where $\lambda_{p}$ is the plasma wavelength and $n_{1}$ and $n_{2}$ are parameters which depend on geometry and material properties [49]. The effective wavelength scaling is based on the propagation constant of plasmons along a long rod essentially using that in a Fabry-Pérot cavity-like model. This model provides an intuitive explanation of the resonance red-shift of thin (width $<20 \mathrm{~nm}$ ) optical antennas. Variations in wire diameter are incorporated when they can be considered adiabatic, which is clearly not the case for the studied width modulated antennas where the width varies on a scale below the wavelength of the plasmon. Using this theory to describe the resonance shifts we observe, is therefore not an option.

\subsubsection{The relation between E-fields and $\epsilon$ of an antenna}

The numerical results depicted in Figure 3.2 show a monotonic scaling of field enhancement and of the resonance wavelength. It is therefore interesting to consider whether or not an independent optimization of the average field-enhancement and spectrum is possible. Wang et al. [99] derived as a general property of plasmon resonances in the quasit-static limit, a relation between the integrated fields in and outside the antenna and the dielectric constants:

$$
-R e\left\{\epsilon_{m}(\omega) / \epsilon_{d}\right\}=\int_{\Omega_{d}} \overline{E^{2}} d V / \int_{\Omega_{m}} \overline{E^{2}} d V
$$


Here $\epsilon_{m}$ and $\epsilon_{d}$ are the dielectric constant of the metal and dielectric, respectively. The real part of the dielectric constant of metal, such as gold and silver, is negative and monotonically decreasing with wavelength. Therefore relation (3.4) signifies that for structures that have the same volume, a redshift corresponds to a relatively lower penetration of the field into the metal, and/or a higher field outside the material. We tested this relationship for our calculated antennas with all phase values and amplitudes, by integrating the square of the electric field in the cross-sectional surface, as depicted in Figure 3.3 (A-E). Though the equality in Equation 3.4 holds only for the volume integrated fields, a linear proportionality is expected to hold for the fields in the cross section area. Figure 3.7 depicts the relationship which is indeed found to be linear up to a reasonable level, indicated by the blue dashed line, which shows a linear fit of the data points. The antenna shapes which lie systematically above the fitted line, are those which show a strong intensity ratio ${\overline{E^{2}}}_{\text {dielectric }} /{\overline{E^{2}}}_{\text {metal }}$, which are associated with the concave antenna shapes. This effect is attributed to local artifacts in the calculations of the fields in our simulations, which is also referred to as the lightning rod effect. In short, this means that strongly curved surfaces do not contain sufficient meshing, leading to non-physical strong local fields. However, it seems this effect is relatively small as the the bulk of the data points follow the linear fit.

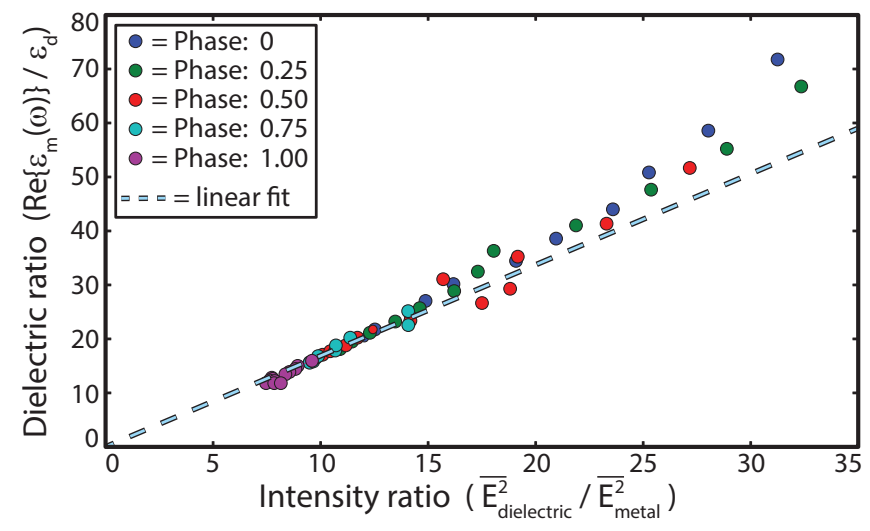

Figure 3.7: Relation between E-fields and $\epsilon$ of an optical antenna. The ratio of dielectric constants in and outside the antenna, versus the ratio of the electric field in and outside the antenna, for simulated antennas with different modulation phases $(0,1 / 4,1 / 2,3 / 4,1) \pi$ and amplitudes $(0-30 \mathrm{~nm})$. The dashed line shows a linear fit. 
We should stress here that Equation 3.4 does not contain geometric information, and thus only gives an implicit relationship between shape and spectrum or near-field. We can, however, conclude that independent tuning of near-field outside the particle and the spectrum would require substantial variation in the field inside the particle. For a particle of given volume and given the fabrication constraints, variation in the depth of penetration of fields into the structure seems to be quite limited, as most dimensions here are already on the scale of the field penetration (skin) $\operatorname{depth}\left(\delta=\lambda /\left(2 \pi \sqrt{-\epsilon_{m}^{\prime}}\right)=25-30 \mathrm{~nm}\right)$. We see this confirmed in the calculations on our set of particles, thus strongly suggesting resonance wavelength and near-field are not separately tuneable.

This means that by controlling the ratio of the fields outside and inside the antenna, one could tune the resonance wavelength of an antenna. Because the variation of the fields inside the antenna are relatively invariant to the antenna shapes we have investigated, tuning of the resonance wavelength could be obtained by tuning the antenna shape, such that more field is generated outside the antenna. From classical electrodynamics we know that the amount of charge, and thus also field which is generated on/around a metal interface, depends on the angle of the interface with respect to the vector of the driving field, as given by the integral from of Gauss's law: $\oint \vec{E} \cdot d \vec{A}=\frac{Q}{\epsilon_{0}}$ [10]. This is also how the field distribution of an optical antenna is determined in the first order approximation (see Section 3.4.2 and Figure 3.6). We therefore hypothesize a design rule, where the resonance wavelength of an optical antenna can be controlled by increasing the fields in the dielectric material which surrounds the antenna. This could be done by changing the amount of surface area which is orientated orthogonal with respect to the vector of the driving field, as governed by Gauss's law. This hypothesis seems to be valid for the modulated antennas we study, and could be generally true.

To see if this concept also applies on other antenna shapes, the resonance behavior of an H-shaped structure is shown, see Figure 3.8, where we vary the height (A) and the width (B) of the arms. The base of the $\mathrm{H}$-structure, when the height of the arms are equal to zero, consists of a bar structure which has the same dimensions as the unmodulated bar antenna in Figure 3.1. In Figure 3.8 (A) we observe that structures with a constant arm width of $30 \mathrm{~nm}$, and an increasing arm height, show a strong resonance redshift up till $600 \mathrm{~nm}$. This result is very similar to what we have seen for the modulated antenna. However, increasing the width of the arms (B), so that the gaps between the arms reduce 

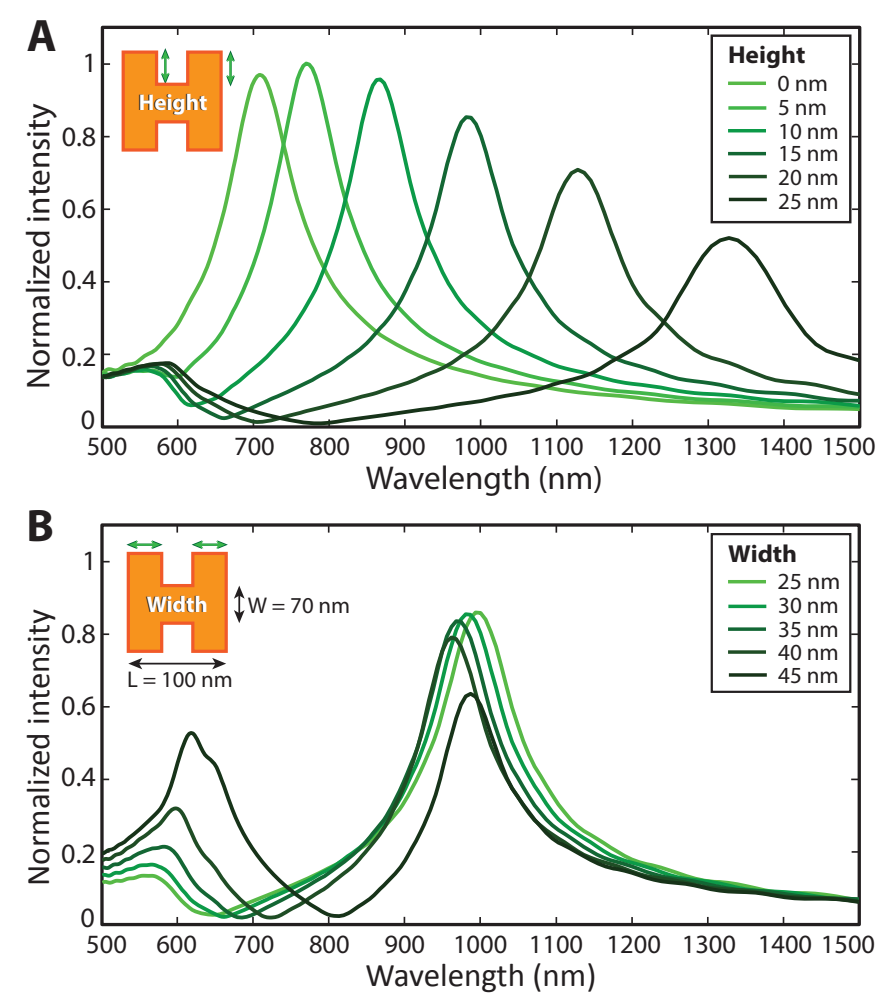

Figure 3.8: Numerically calculated scattering spectra of $\mathbf{H}$-antennas with varying arm height and width. Numerically calculated scattering spectra of $\mathrm{H}$ structures where the height $(\mathbf{A})$ and width (B) of the arms forming the $\mathrm{H}$ shape are varied, indicated with the green arrows. The $\mathrm{H}$-structure is built up from a simple bar structure, having a length and width of 100 and $70 \mathrm{~nm}$, respectively, where the arms in (A) have a width of $30 \mathrm{~nm}$ and have a height of $15 \mathrm{~nm}$ in (B). A linearly polarized illumination source is used, which is orientated parallel along the central bar of the antenna.

to only $10 \mathrm{~nm}$, hardly changes the resonance peak at $1000 \mathrm{~nm}$. This clearly shows that the model, which is based on calculating the potential energy, does not work for this structure, as this model predicts that the resonance frequency is strongly dependent on the separation of the surface charges. Figure 3.8 nicely illustrates that the resonance behavior of an optical antenna is very sensitive to perturbation at specific positions, while being almost insensitive to perturbations at other locations. In both this structure and the modulated antennas we observe a strong redshift of the resonance wavelength, when the structures contain more surface area which is orientated orthogonal with respect to the vector of the driving field. 


\subsection{Asymmetric modulations}

Finally we investigate how the antenna resonances change if the two sides of the antenna have a different sinusoidal modulation amplitude, thus introducing an asymmetry in the antenna. The starting structure is a modulated antenna having a modulation phase of 0 . The asymmetry is realized by shifting the center section of the antenna. The volume is again kept constant for the different contour shapes. Figure 3.9 (A) shows one experimental and several numerical scattering spectra of antennas with different degrees of asymmetry in their respective contour shapes. The antennas, having a center shift of 0 to $30 \mathrm{~nm}$, change from a fully symmetric antenna to an antenna with a large asymmetry, where one of the sides is flat. The fabricated asymmetric antenna has a center shift of $10 \mathrm{~nm}$. The numerical scattering spectra reveal that,
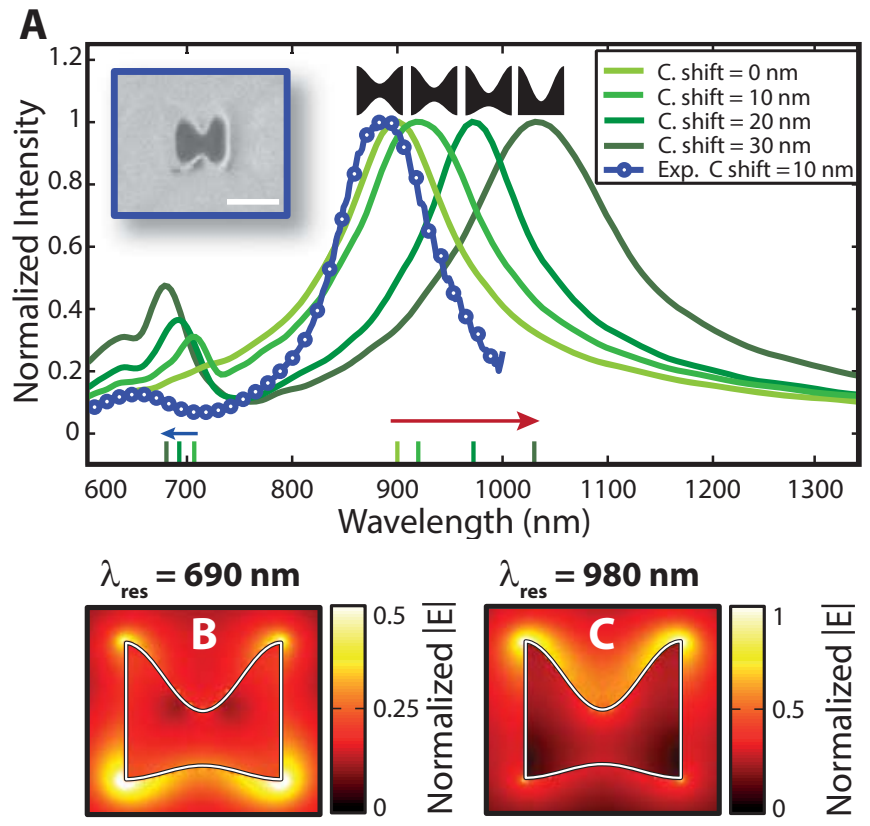

Figure 3.9: Asymmetric width modulated antennas. (A) experimental and numerical scattering spectra of asymmetric width modulated antennas, having different degrees of asymmetry due to a vertical shift of the center section. The calculations have been done for a starting modulation amplitude of $15 \mathrm{~nm}$. Micrograph showing a SEM image of the experimented structure, with a white bar indicating $100 \mathrm{~nm}$. (B-C) numerically calculated electric field distributions of an asymmetric width modulated antenna, with a center shift of $20 \mathrm{~nm}$ at $\lambda_{\text {res }}=690$ $\mathrm{nm}$ and $\lambda_{\text {res }}=980 \mathrm{~nm}$. The electric field distributions are normalized to the maximum value of figure $(\mathrm{C})$. 
upon introducing asymmetry in the contour shape, the single resonance peak of the symmetric structure is significantly redshifting, as can be expected based on the effect of amplitude of the modulation we found for the symmetric antennas. A second peak emerges around $700 \mathrm{~nm}$, which is blueshifting. The two resonance peaks are also observed in our experimental scattering spectra, although it is slightly more blueshifted. The near-field distributions of the two modes of an asymmetric modulated antenna with a center shift of $20 \mathrm{~nm}$, are shown in (B,C) at their resonance wavelength $\lambda_{\text {res }}=690 \mathrm{~nm}$ and $\lambda_{\text {res }}=980 \mathrm{~nm}$, respectively. Interestingly the near-fields of both the resonances show to be predominantly present on either of the modulated sides of the antenna, where the shorter and longer wavelength resonance are localized on the side with smaller and larger modulation amplitude, respectively. In Figure 3.10 we calculate the far-field scattering patterns of both resonances, shown in Figure 3.9 (B,C). Surprisingly a dipolar far-field scattering pattern is found for the resonance at $\lambda_{\text {res }}=980 \mathrm{~nm}$, even though the structure is highly asymmetric and the near-fields are strongly localized on one modulated surface of the antenna. Butet et al. [45] has shown a similar behavior, where he studied the effects of small shape deformations on the radiation pattern of a dimer. The deformations which are
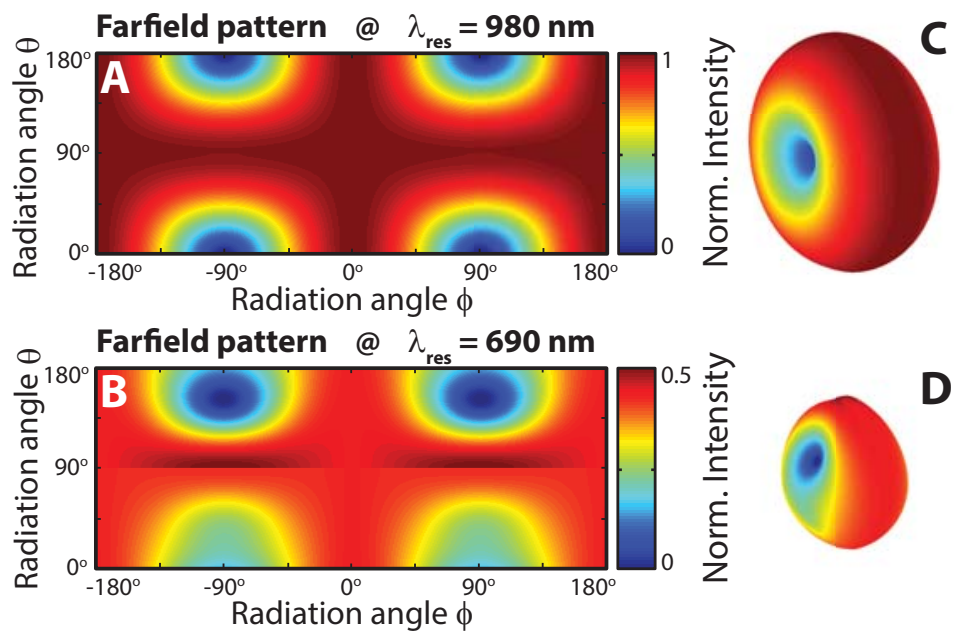

Figure 3.10: Far-field scattering patterns of asymmetric width modulated antennas. (A-D) Numerical calculations of the far-field radiation pattern of an asymmetric width modulated antenna with a modulation amplitude and center shift of 15 and $20 \mathrm{~nm}$, respectively. $(A, C)$ and $(B, D)$ show the far-field patterns at the two resonant wavelengths $\left(\lambda_{\text {res }}=980 \mathrm{~nm}\right.$ and $\left.\lambda_{\text {res }}=690 \mathrm{~nm}\right)$, where excitation source is polarized parallel to the longitudinal axis of the antenna. 
applied in the asymmetric structures, however, far exceed those studied by Butet. Despite the strong anti-phased charge distributions, the asymmetric antennas still show a strong dipolar scattering pattern. The lower wavelength resonance turns out to have clear deviations from a dipolar scattering pattern, indicating it is a higher order mode. These structures can have interesting applications, for instance in non-linear mixing processes, where it is useful to have a single antenna exhibiting two resonances.

\subsection{Conclusions}

The relation between the shape and the resonance of an antenna has been experimentally and numerically studied, using an antenna design which shows a gradual transition between a concave and convex shape. The optical antennas we have studied, show a sinusoidal modulation in width. By tuning the modulation phase and amplitude, the resonance wavelength can be varied over an impressive range of $600 \mathrm{~nm}$. Four commonly used models are considered in an attempt to find an intuitive relation between the shape of the modulated antennas and their resonance behavior. Although many models are able to relate the electric field distribution of an antenna with their resonance wavelength, they do not provide a generalized relation between the antenna shape and their resonance behavior. However, the last model we have reviewed shows that the resonance wavelength of an antenna, regardless of shape or size, is directly related to the ratio of the fields in and outside the antenna. This suggest that by controlling the ratio of the fields outside and inside the antenna, one could tune the resonance wavelength of an antenna. In our calculations of the various modulated antennas, we find consistently that the fields inside the antennas only slightly changes, in contrast to the fields outside the antennas.

We therefore hypothesize a design rule in which the resonance wavelength of an optical antenna can be controlled by changing the amount of surface area, which is orientated orthogonal with respect of the vector of the driving field. This seems to be valid for modulated antennas and could be generally true. Lastly, we have introduced asymmetry in the modulated antenna so that the two longitudinal sides have different modulation amplitudes. Consequently two resonances are formed. The presented antenna designs are interesting for the use in many optical processes where the resonance wavelength needs to be tuned over a large bandwidth, and in non-linear mixing processes, where it could be beneficial to have a single antenna exhibiting two resonances. 
Chapter

\section{Coupling optical antennas}

In many optical applications there is a need for controlling the angle dependent emission or absorption. For instance a hybrid system of an LED and arrays of optical antennas are proposed to replace the bulky diffraction and mirror systems which are used nowadays [100]. The findings presented in this chapter open an additional path to control the far-field scattering patterns of two-antenna system. We investigate the coupling of two optical antennas having the same resonance frequency, but different polarizability and/or Q-factors. Three different optical antenna systems are explored, each showing a strong polarizability redistribution for the symmetric eigenmodes of the coupled systems. A modified coupled harmonic oscillator (CHO) model is presented, which incorporates a mechanical equivalence to the polarizability and Q-factor of an optical antenna. This modified CHO model shows that the mechanical amplitude or optical polarizability of the individual oscillators are strongly dependent on the symmetry of the eigenmode of the coupled system.

\subsection{Introduction}

In Chapter 3 we have shown how the properties of the localized plasmon resonances are affected by the geometry of an optical antenna. Simple geometries such as rods, spheres, triangles and disks exhibit pronounced dipolar modes, where the corresponding resonance frequencies can be widely tuned over the visible and near-infrared spectrum by changing their physical size and shape [20,33,101,102]. Nanostructures, consisting of multiple particles, show a richer resonance behavior. Due to the coupling of plasmons of the individual structures, symmetric and antisymmetric eigenmodes are formed $[20,61,103]$. Of specific interest are nanostructures that exhibit modes dominated by higher-order polariz- 
abilities [104-107]. These modes typically have reduced radiative damping, due to the less efficient coupling of higher order polarizabilities to far-field radiation, and are therefore often called 'dark' modes [105-109]. Studies on the coupling of dark and bright modes have shown that bright modes can destructively interfere with dark modes, a phenomenon which in literature has also been called electromagnetic induced transparency, or Fano-type interference $[57,58,110,111]$. Increased scattering directivity due to the presence of these Fano-type resonances, seen in single and coupled resonator systems, has been observed [112]. Although Fano-type resonances have been widely investigated, and antennas with different polarizability (dark and bright modes) have been coupled, the question of how the polarizability ratio of the antennas is affected by being in a coupled state,has, to the best of our knowledge, not yet been explored.

The collective oscillatory behavior of free electrons in a coupled twoantenna system can be described by the movement of a harmonicallydriven mass-spring system, which has often been used to describe the resonant behavior of coupled optical antenna systems $[98,113]$. In this mechanical model, the two resonances, which are associated with the coupling of two oscillators, are described by a linear combination of the two oscillators, having different amplitudes and phases.

In this chapter the coupling of two antennas having different polarizabilities is studied, where the emphasis is on determining the redistribution of the polarizability ratios of the antennas as a function of the symmetry of the eigenmodes of the coupled system. First the scattering spectra of a coupled bar-ring antenna system are experimentally and numerically investigated, where the bar and ring exhibit a dipolar and hexapolar mode profile. Next, the angle dependent scattering patterns of the symmetric and anti-symmetric eigenmodes of the coupled system are numerically studied. We demonstrate that the angle dependent scattering patterns of the two eigenmodes of a coupled system are distinctly different, which suggest that the polarizability ratio of the two antennas, which are coupled, is strongly dependent on the symmetry of the eigenmodes. In the third section, an intuitive model is used to reconstruct the modeprofiles of the individual bar and ring antennas, which are then used to estimate the polarizability ratio of the bar and ring antennas when they are symmetrically and anti-symmetrically coupled. In order to show that this phenomena is also occurring in other coupled systems, the current density distributions of two other coupled two-antenna systems are shown. Finally, a modified coupled harmonic oscillator model is introduced with which the angle dependent scatter- 
ing patterns of two antennas having different polarizability and Q-factor ratios are explained.

\subsection{Coupled bar-ring system}

In this section, the coupled bar-ring system is chosen as a case study (see Figure 4.1), because the coupled system consists of two antennas having very different polarizabilities and angle-dependent scattering patterns $[114,115]$. The far-field angle dependent scattering patterns of optical antennas are directly related to the current distributions in the antenna [23]. Whereas the bar antenna has a strong dipole character, the ring antenna is tuned to contain a hexapole ring mode. As a consequence of the destructive nature of the current distribution of a hexapole ring mode, its polarizability is approximately 3.5 times lower compared to the bar antenna it is coupled with. In the first part of this section, the coupling induced spectral behavior is experimentally and numerically in-
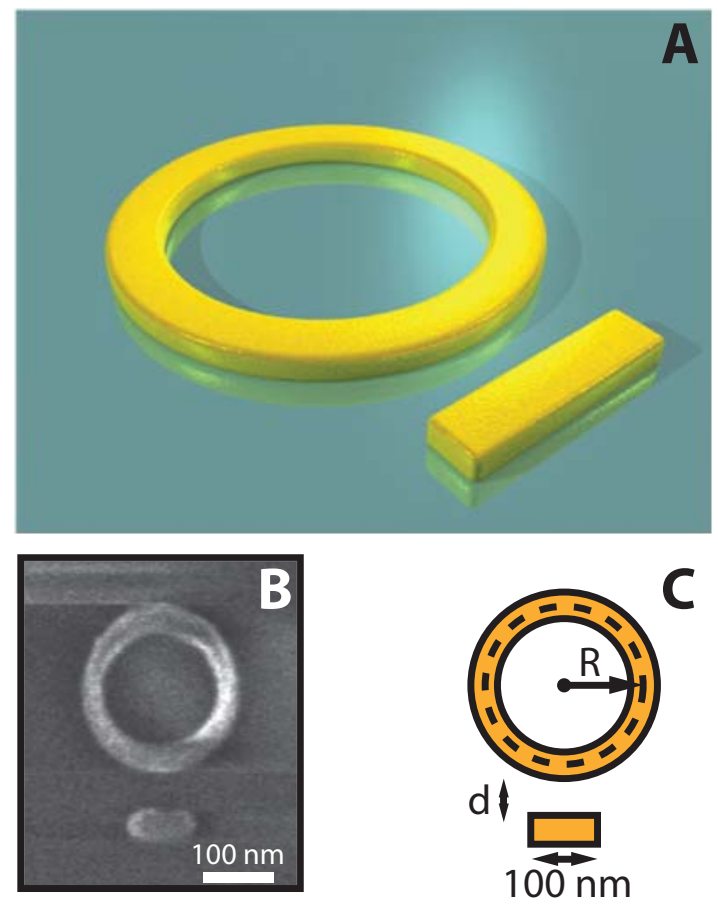

Figure 4.1: Bar-ring geometry. $(A)$ a $3 D$ rendering of the investigated structure. (B) a scanning electron micrograph of a typical e-beam fabricated bar-ring sample. (C) geometry and labeling convention for bar-ring structures: $\mathrm{R}$ denotes the ring radius and $d$ is the separation distance between the bar and the ring. 
vestigated. The angle dependent scattering patterns of the eigenmodes are then numerically studied and compared with a dipole model, which provides an intuitive link between current distributions in an antenna and the resulting far-field scattering patterns.

A selection of numerically calculated scattering spectra of an individual bar and ring antenna, illuminated under different angles of incidence $(\alpha)$, is shown in Figure 4.2. The numerical calculations of the scattering intensity where performed using a finite element method technique; the details of the method which is used to obtain the scattering cross intensity spectra are described in Appendix B. The scattering spectrum of the ring antenna shows a strong angle dependence with the emergence of two extra resonances for illumination angles $\alpha<90^{\circ}$. Panels 1-3 of Figure 4.2 illustrate the $E_{z}$ field distributions of the three ring modes, showing their dipole, quadrupole and hexapole nature. The relative scattering cross sections of the higher-order ring modes reduce significantly with increasing multipole moment, where the maximum scattering cross section of the quadrupole and hexapole ring mode are respectively 3 and 12 times lower than the dipole bar mode. The hexapole ring mode is most efficiently excited at an angle of $\alpha \approx 35^{\circ}$ degrees incidence. 


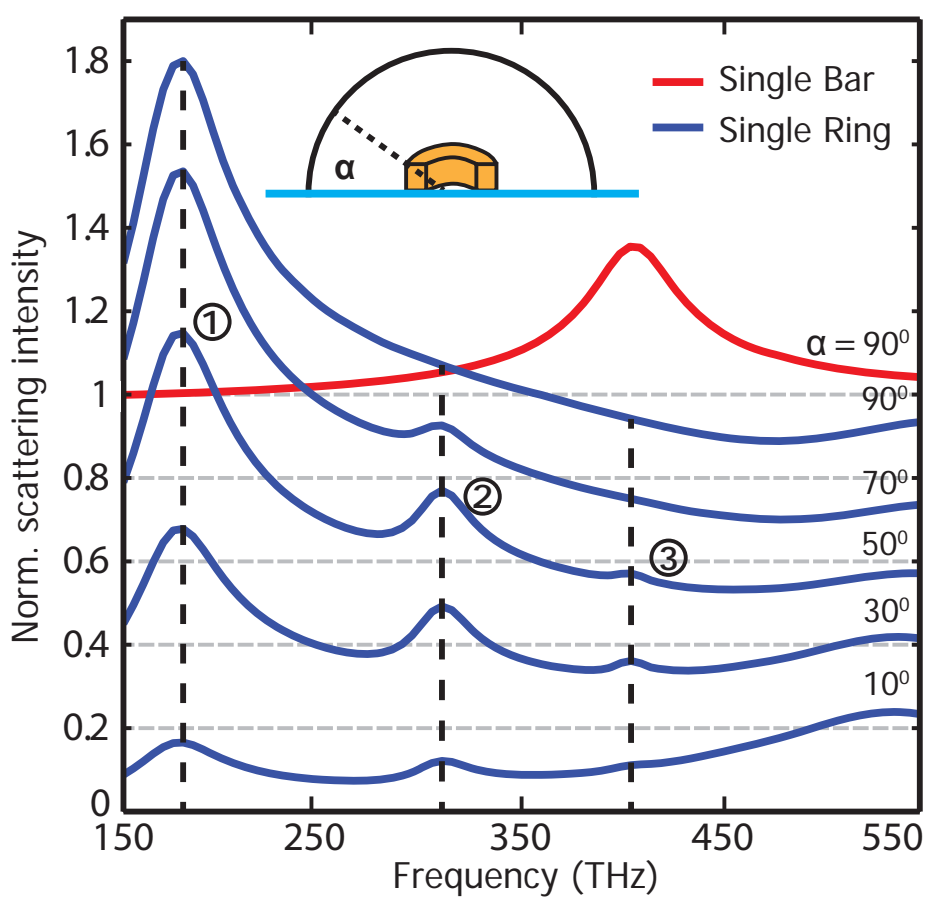

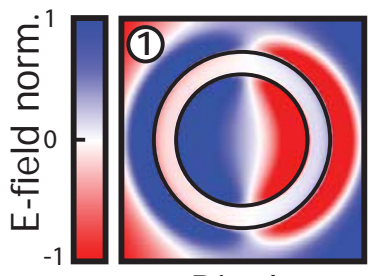

Dipole

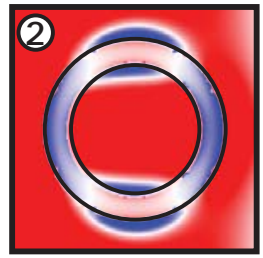

Quadrupole

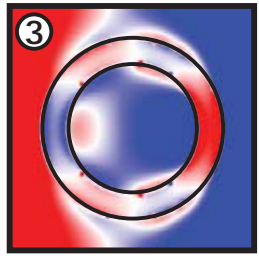

Hexapole

Figure 4.2: Scattering intensity spectra of individual bar and ring antennas. (Above) numerical calculations of the scattering intensity of a single bar antenna (red) and single ring antenna (blue) with a ring radius of $150 \mathrm{~nm}$, excited under different angles with respect to the substrate surface. (1-3) show snapshots in time of the $E_{z}$ field distribution, corresponding to the resonance frequencies indicated in the panel above. 
The polar plot in Figure 4.3 further illustrates the angle dependent scattering for the dipole bar and the hexapole ring mode at their resonance frequency. In these numerical calculations, the polarization angle of the excitation source is set parallel to the long axis of the bar resonator, and two configurations of illumination are chosen: (1), in which the angle of illumination is varied around the short axis of the bar resonator, or (2), where the angle of illumination is varied around the long axis of the bar resonator. The polar plot are obtained by numerically calculating the scattering spectra under different angles of illuminations and extracting the amplitudes of the resonance peaks. The scattering pattern of the bar and the hexapole ring mode differ strongly. The scattering pattern of the bar resonator has a dipole character, resembling a donut-like shape. The scattering pattern of the hexapole ring mode is circular symmetric and shows directional scattering with a maximum scattering intensity at $35^{\circ}$.

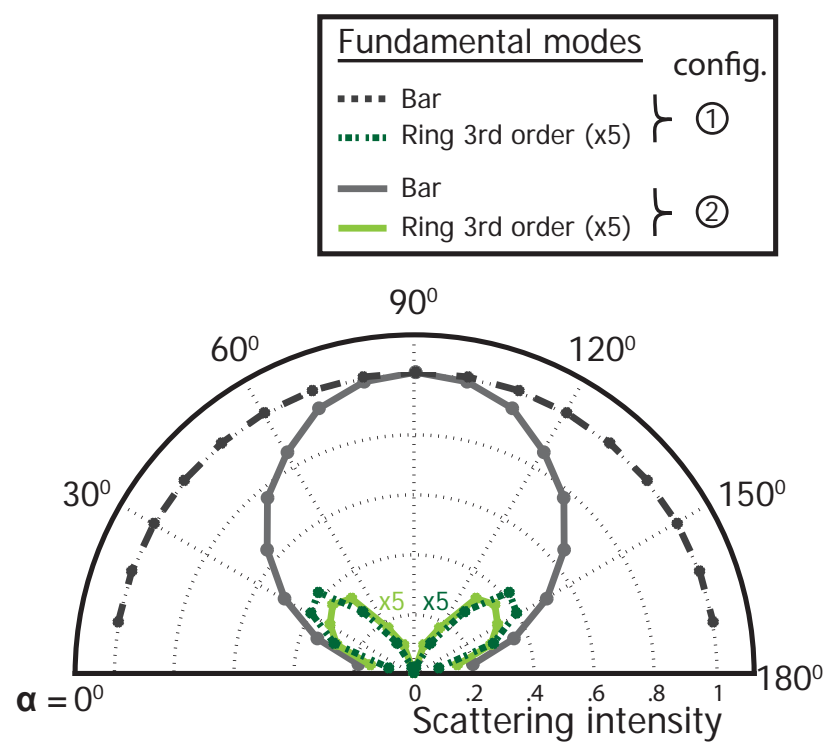

Figure 4.3: Numerically calculated angle-dependent scattering pattern of an individual bar and ring. Numerically calculated polar plot of angle-dependent scattering pattern of a dipolar bar mode, indicated with light and dark gray curves, and of a hexapolar ring mode, indicated in green and magnified by a factor 5 . Two configurations of illumination are chosen: 1 in which the angle of illumination is varied around the short axis of the bar resonator, or 2 where the angle of illumination is varied around the long axis of the bar resonator. 
Now, the coupling of the bar and ring antenna is considered. Figure 4.4 shows experimental data and numerical calculations of bar-ring resonators with decreasing inter particle distances starting with a separation of $50 \mathrm{~nm}$. The experimental scattering spectra are collected using the dark-field micro-spectroscopy setup described in Section 2.1, and are normalized to maximum scattering intensity of the scattering spectra where the particle spacing is $50 \mathrm{~nm}$. Scattering spectra are plotted for the incident light polarized perpendicular (red dotted) or parallel (solid) with respect to the long axis of the bar. When the structure is illuminated with perpendicularly polarized light, a very weak resonance peak is observed, which is attributed to the scattering of the hexapole ring mode. Similar scattering spectra, with perpendicular polarized illumination, are observed for structures with smaller interparticle distances. Even when the two resonators are strongly coupled, the eigenmodes show to be weakly polarizable for perpendicular polarized illumination, similarly to the uncoupled situation. This result suggests that even in the coupled situation, the eigenmodes can be described as a linear combination of the two individual resonator modes. For parallel incident light, both experimental (A) and numerical (B) scattering intensities show an asymmetric splitting of a single resonance peak for increasing coupling. Since this splitting is wider than the widths of the original resonances, the system with a gap size $<10 \mathrm{~nm}$ can be considered to be in the strong coupling regime. The overall broader resonance peak observed in the experimental data, is attributed to the chrome adhesion layer of $0.5 \mathrm{~nm}$, used for the fabrication of the nanostructures [116]. The calculations reveal that the two eigenmodes are anti-symmetric and symmetric in nature, see Figure 4.4 panels B1 and B3, respectively. The black arrows in these panels indicate the presence of a phase flip, which highlights the in- and out-of-phase behavior of the two coupled antennas. The field distribution, shown in Figure 4.4 panel B2, shows similarities to both of the modes in panels B1 and B3, but with a greatly reduced field strength, which is due to the destructive interference of the two fundamental modes. Note that in both the experimental and numerical scattering spectra the symmetric eigenmode is barely blue-shifted with increasing coupling in comparison to the anti-symmetric mode which strongly red-shifts. 

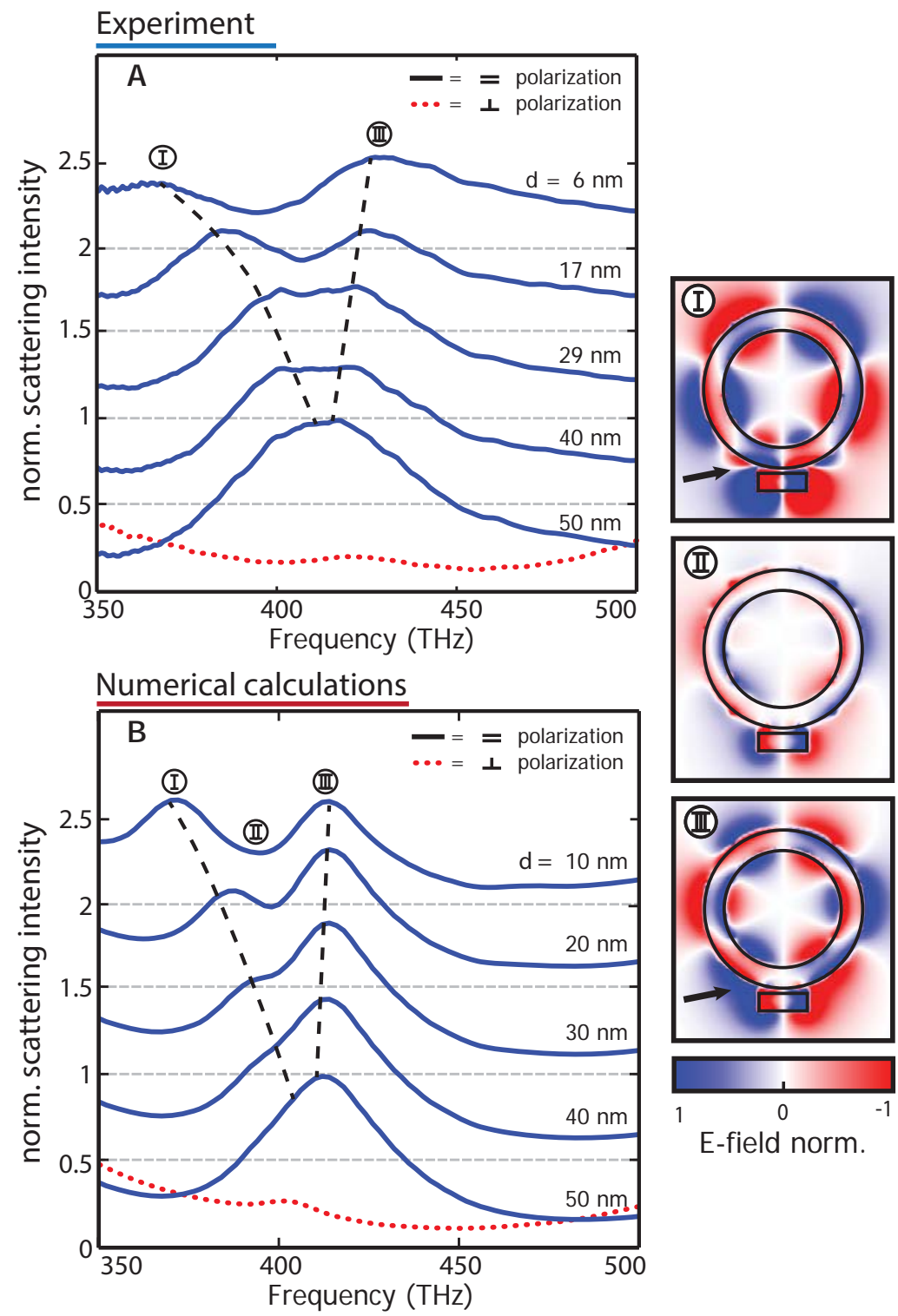

Figure 4.4: Spectral behavior of coupled bar-ring antennas. Experimental (A) and numerically calculated (B) scattering spectra for various bar-ring resonators, with different separation distances. Scattering spectra are plotted for excitation light polarized parallel (solid) and perpendicular (red dotted) with respect to the long axis of the bar. The gray dashed lines indicate the offset for the different spectra. (B1-3) show the z-component of the numerically calculated electric field distribution at the given frequencies for a bar-ring resonator with a separation distance of $10 \mathrm{~nm}$. The black arrows highlight the in-and out-of-phase character of the two eigenmodes of the coupled system. 
The far-field scattering pattern of a coupled bar-ring system with a 10 $n m$ interparticle distance is numerically calculated, and shown in Figure 4.5. The scattering patterns are obtained by acquiring the amplitude of the two eigenmodes, obtained from the numerically calculated scattering spectra, for various illumination angles. The scattering patterns of the two eigenmodes show very distinct behavior, where the anti-symmetric eigenmode has a donut-like scattering pattern resembling a dipole-like mode. The scattering pattern of the symmetric mode
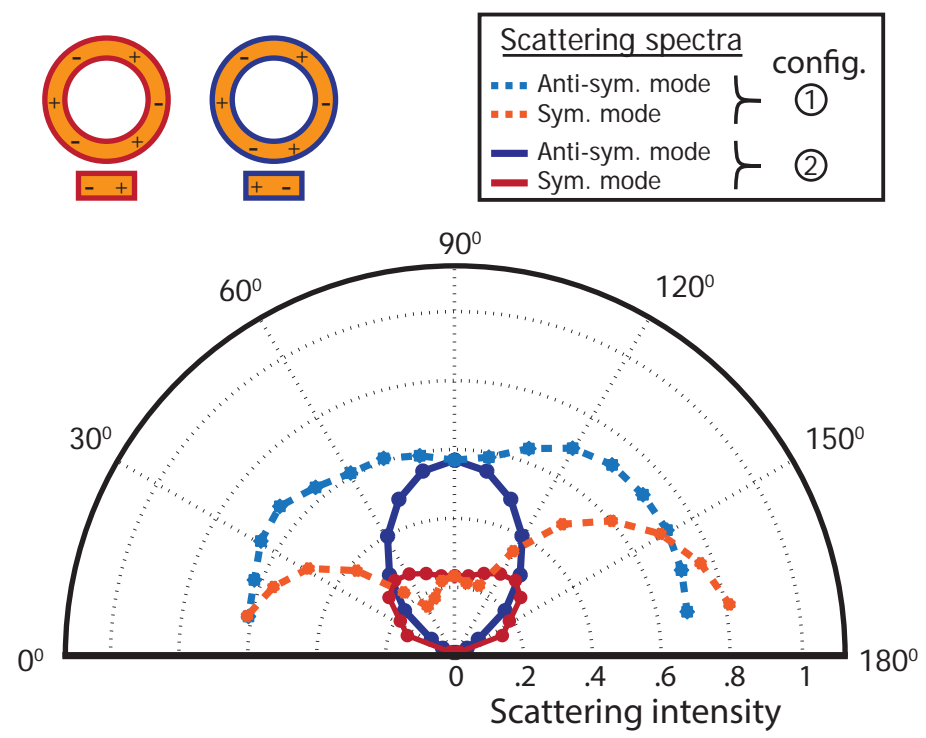

Figure 4.5: Numerically calculated angle dependent scattering pattern of a coupled bar-ring system. Numerically calculated polar plot of angle-dependent scattering pattern of a coupled bar-ring system with a $10 \mathrm{~nm}$ spacing. The symmetric and anti-symmetric eigenmodes are indicated in red and blue, respectively. Two configurations of illumination are chosen: 1 in which the angle of illumination is varied around the short axis of the bar resonator, or 2 where the angle of illumination is varied around the long axis of the bar resonator.

is very different and shows more similarities to the scattering pattern of the hexapole-like mode, which can be seen in Figure 4.7, although a bit less pronounced. This is an intriguing result if one considers that the fundamental scattering intensity of an isolated bar resonator is an order of magnitude larger compared to that of an isolated hexapole ring mode, suggesting that due to the coupling, the polarizability ratio of the antennas is redistributed. 


\subsection{Dipole model}

In an attempt to reconstruct the farfield scattering distributions of the symmetric and anti-symmetric eigenmodes of the coupled bar-ring system, an intuitive reconstruction of the current distributions of the barring modes is done using a combination of dipoles, for which the orientation, amplitude and phase are set accordingly (see Figure 4.6). The two antennas can then be represented separately by two groups of dipoles. Because of the dipole nature of the bar, it can be represented by a single dipole. The hexapole ring antenna is constructed out of six dipoles, which show an alternating $\pi$ phase jump and a $60^{\circ}$ orientation angle shift, forming a circle with radius $r$.
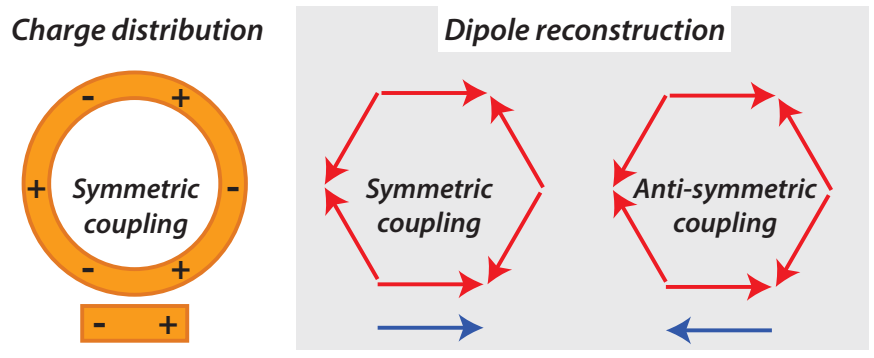

Figure 4.6: Reconstruction of bar and ring modes using multiple dipoles (Left) a cartoon showing the charge distribution of a symmetrically-coupled barring antenna. (Center and right) the reconstruction of the charge distribution of a symmetrically and anti-symmetrically-coupled bar-ring antenna using only dipoles, indicated with blue and red arrows.

The resulting far-field scattering patterns of the two individual antenna are shown in Figure 4.7, showing the 3D renderings of the angle-dependent scattering patterns in A and B. The angle dependent scattering intensity along two orthogonal crosscuts of both the fundamental bar and ring modes, indicated with solid and dashed lines in $\mathrm{A}$ and $\mathrm{B}$, are shown in Figure 4.7 C. In order to compare the far-field patterns of both the numerical calculations and the dipole model, the angle of the crosscuts in Figures 4.7 to 4.9 are chosen so that they coincide with the crosscuts shown in Figure 4.5. Similarities, like the strong scattering along shallow angles, are found when comparing the fundamental hexapole mode in Figure 4.7 with the symmetrically coupled antenna in Figure 4.5.

Now the far-field scattering patterns of a linear combination of the bar and ring modes, are studied in Figure 4.8, which are formed by the two groups of dipoles. The dipoles are positioned in such a way that they most accurately reproduce the dimension of the investigated bar-ring geometry, having an antenna spacing of $10 \mathrm{~nm}$. The symmetric and 

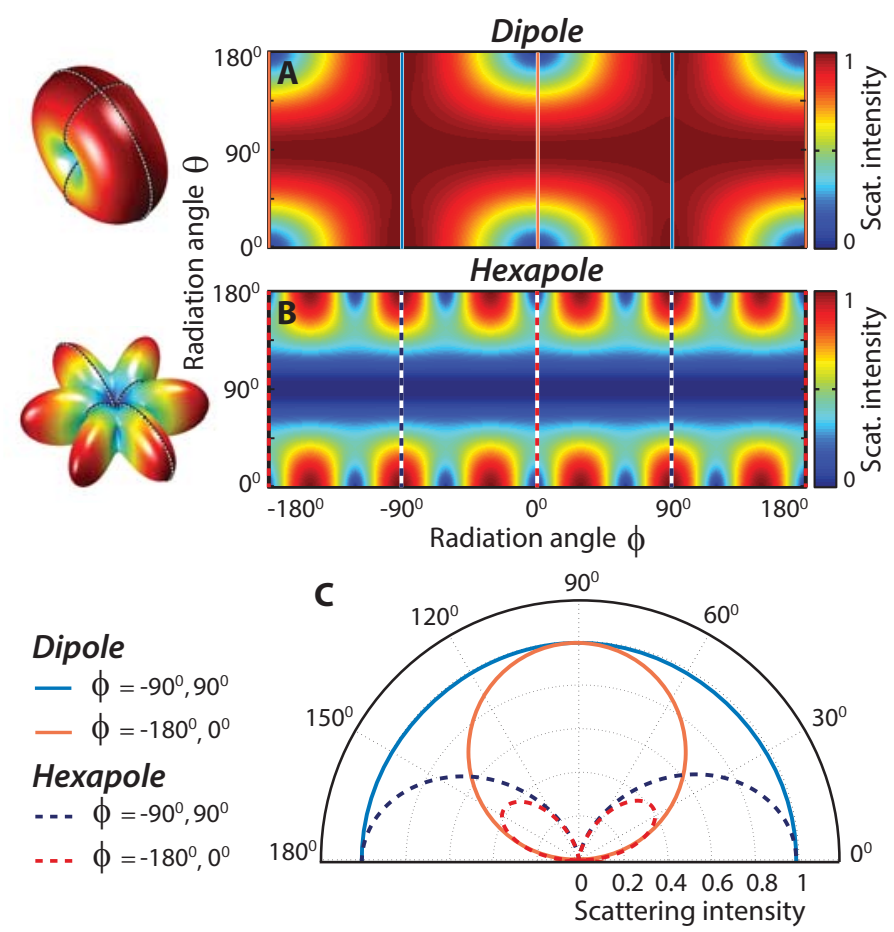

Figure 4.7: Far-field pattern of a dipole and hexapole mode. (A, B) Unwrapped far-field scattering patterns of a single dipole and single hexapole mode; along with a $3 \mathrm{D}$ rendering. Four vertical lines in $(A)$ and $(B)$ at $\phi=-180^{\circ},-90^{\circ}, 0^{\circ}, 90^{\circ}$ are extracted to make a similar far-field representation in the form of a polar plot (C).

anti-symmetric eigenmodes are reproduced by applying a $\pi$ phase to the dipole, which represents the bar antenna. In this intuitive model, coupling between the individual dipoles is not taken into account; rather the constructive and destructive far-field contributions of the dipoles are calculated. This model implicitly assumes that the mode profiles of the fundamental antennas are unaltered due to coupling and only their overall respective amplitudes and phases change. This assumption is not that strange as a similar assumptions is made when using a coupled mechanical oscillator model to describe coupled optical (antenna) system $[98,113]$. Later, this model is used to estimate the ratio of the polarizability of the coupled bar-ring oscillators. The shape of the farfield scattering distributions are highly dependent on the amplitude ratio of the dipole groups, which form the bar and ring modes. The scattering cross section ratios' $\left(\sigma \propto p^{2}\right)$ dependence of the far-field scattering patterns of a bar-ring system is shown in Figure 4.8. The top far-field 


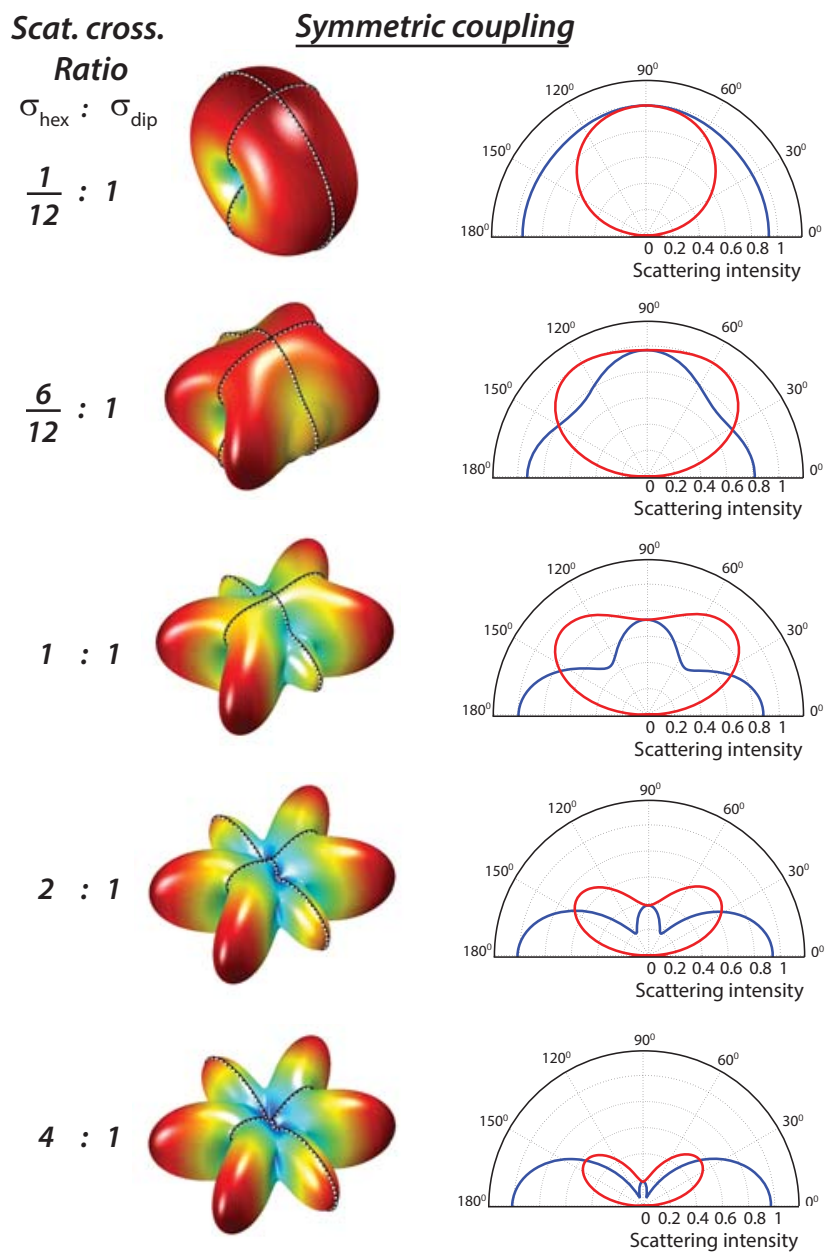

Figure 4.8: Far-field reconstruction coupled dipole and hexapole mode. Farfield reconstructions of coupled dipolar and hexapolar systems presented in 3D renderings and polar plots. From top to bottom the scattering cross section of the hexapolar mode is incrementally multiplied by a factor of 2 .

distribution in Figure 4.8 shows a symmetrically-coupled system, where the bar and ring modes have a scattering cross section ratio comparable to their fundamental scattering cross section ratio, leading to a strong dipole like far-field pattern. Upon increasing the scattering cross section of the hexapole ring mode, the far-field distribution slowly changes to a shape similar to the hexapole mode seen in Figure 4.7. The intermediate far-field scattering distribution results, and especially the 1:1 scattering cross section ratio, are of great interest. Section 4.5.2 describes how for the symmetric eigenmode of a coupled two-antenna system, the polar- 
izability ratio of the antennas will be 1:1, regardless the polarizability ratios of the fundamental modes. Comparing the far-field scattering patterns of Figure 4.8 with those of Figure 4.5, corroborates this finding and suggests that a scattering cross section ratio of $1: 1$ or larger is needed to provide a reasonable match to the far-field scattering patterns seen in Figure 4.5.

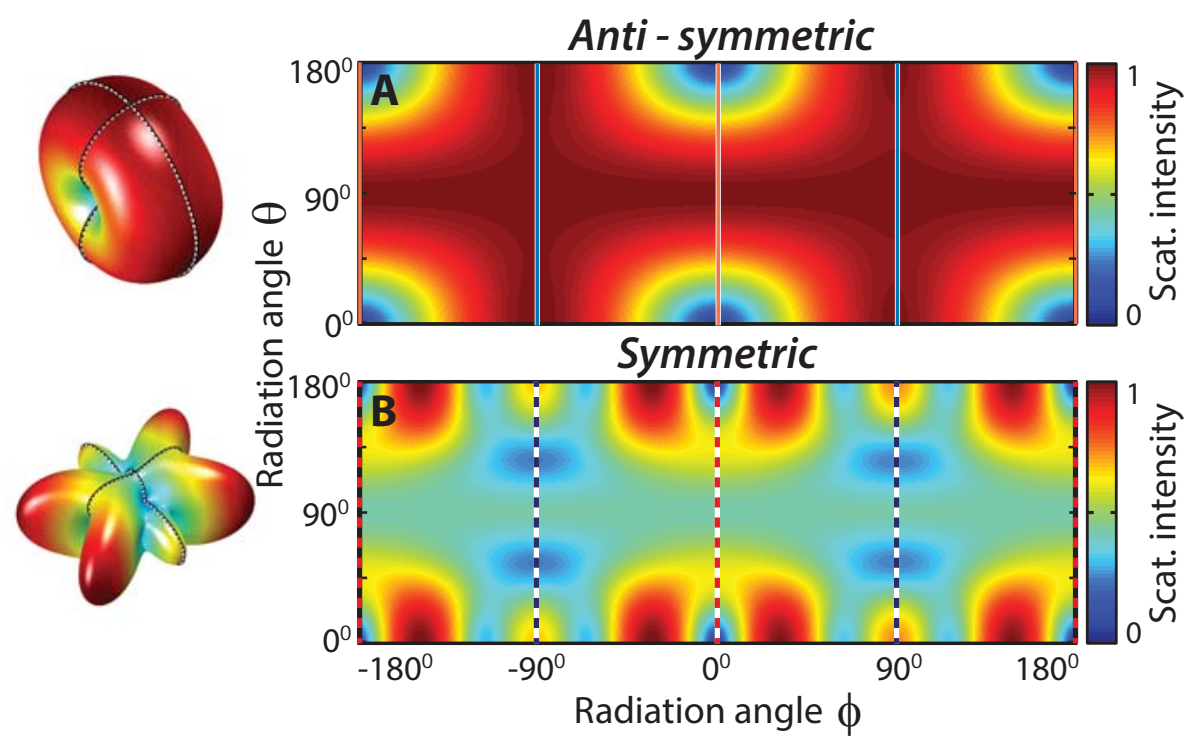

Anti - symmetric

- $\phi=-90^{\circ}, 90^{\circ}$

- $\phi=-180^{\circ}, 0^{0}$

\section{Symmetric}

$\begin{array}{ll}-\ldots & \phi=-90^{\circ}, 90^{\circ} \\ ---\phi & =-180^{\circ}, 0^{\circ}\end{array}$

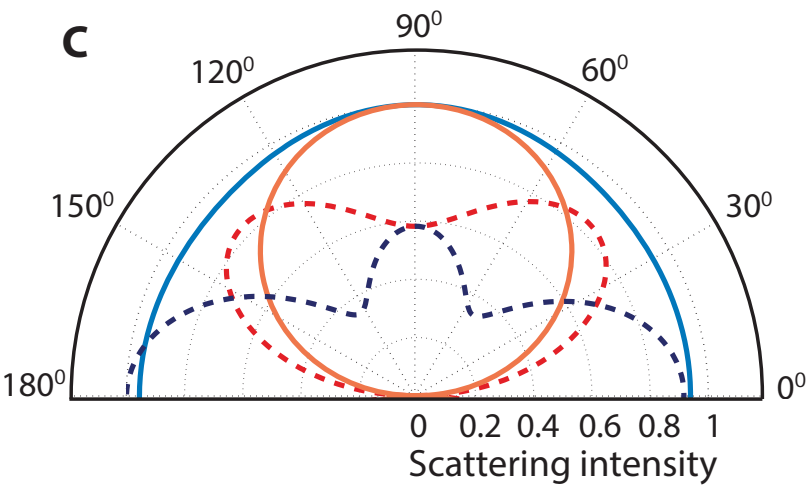

Figure 4.9: Far-field reconstruction of a coupled dipole hexapole system, using the $\mathrm{CHO}$ model. (A, B) Unwrapped far-field scattering patterns of a symmetric and anti-symmetric coupled bar-ring system, using the amplitude ratios, obtained from the CHO-model (see Section 4.5.2); along with a 3D rendering. Four vertical lines in (A),(B) at $\phi=-180^{\circ},-90^{\circ}, 0^{\circ}, 90^{\circ}$ are extracted to make a similar far-field rendering in the form of a polar plot $(\mathbf{C})$. 
The polarizability ratios calculated with the $\mathrm{CHO}$ model which is described in Section 4.5.2 are used to construct the far-field distributions of both the symmetric and anti-symmetric eigenmodes, shown in Figure 4.9. When comparing the far-field scattering pattern of the polar plot of Figure 4.9 with those in seen in Figure 4.5, a couple of clear similarities become apparent. Both the anti-symmetric modes, in the numerical calculations and the dipole model, show a clear dipole far-field scattering pattern. Also, the basic shape of the symmetric mode in Figure 4.5, can clearly be distinguished in the far-field pattern of Figure 4.9. Although the ratios of the dashed red and blue lines in Figure 4.9 are not perfectly matched to those of Figure 4.5, the overall shapes compare very well. A better match between the far-field scattering pattern of the dipole model of Figure 4.9, and those obtained from numerical calculations in Figure 4.5 can probably be obtained by finding a more accurate match of the current distributions. This could be done by tweaking the amplitude phase and positions of the dipoles which form the two antennas, however the goal of this model was not to perfectly reconstruct the current distributions using multiple dipoles, but to provide an intuitive and simple reconstruction, while still maintaining physical meaning.

\subsection{Coupling of other two-antenna systems}

In this section numerically calculated scattering spectra and current density distributions of two other coupled two-antenna systems are shown to illustrate that the redistribution of the polarizability, which is seen in the bar-ring system is also occurring in other coupled antenna systems.

The second investigated optical system consists of two coupled spheres having different radii $r=(50: 100 \mathrm{~nm})$, resulting in a polarizability ratio $\mathbf{R}_{p 1,2}^{\prime}=8$ [23]. This simple system is a nice point to start our investigation as the polarizability of a sphere antenna scales linearly with its volume $\alpha(\omega)=4 \pi \epsilon_{0} r^{3} \frac{\epsilon_{1}-\epsilon_{2}}{\epsilon_{1}+2 \epsilon_{2}}$, assuming $r<\lambda$. The scattering spectra of the coupled and uncoupled system spectra are shown in Figure 4.10, revealing a single peak in the uncoupled state and two peaks in the coupled state. The magnitude and phase of the current density plot show that the eigenmode at $735 \mathrm{THz}$ is anti-symmetric in nature, and shows an almost equal current density magnitude for both the antennas' crosscuts. As the polarizability scales with the number of electrons $N$, the polarizability, and thus amplitudes, of the two antennas in the anti-symmetric mode show an unequal ratio, which scales with their volume. However looking at the peak at $804 \mathrm{THz}$ a symmetric eigenmode is revealed, and as predicted in our modified $\mathrm{CHO}$ model, the current 
density of the smaller particle is greatly enhanced. Integrating over the whole volume of the two-sphere system, a current ratio of approximately 1:2 for the small vs big sphere is found, meaning a fourfold fold magnification of the small sphere's polarizability. This system has also been studied by Lippitz et all. [117], and they also found that the signal of the small sphere is greatly enhanced for the symmetric eigenmode of the coupled system.

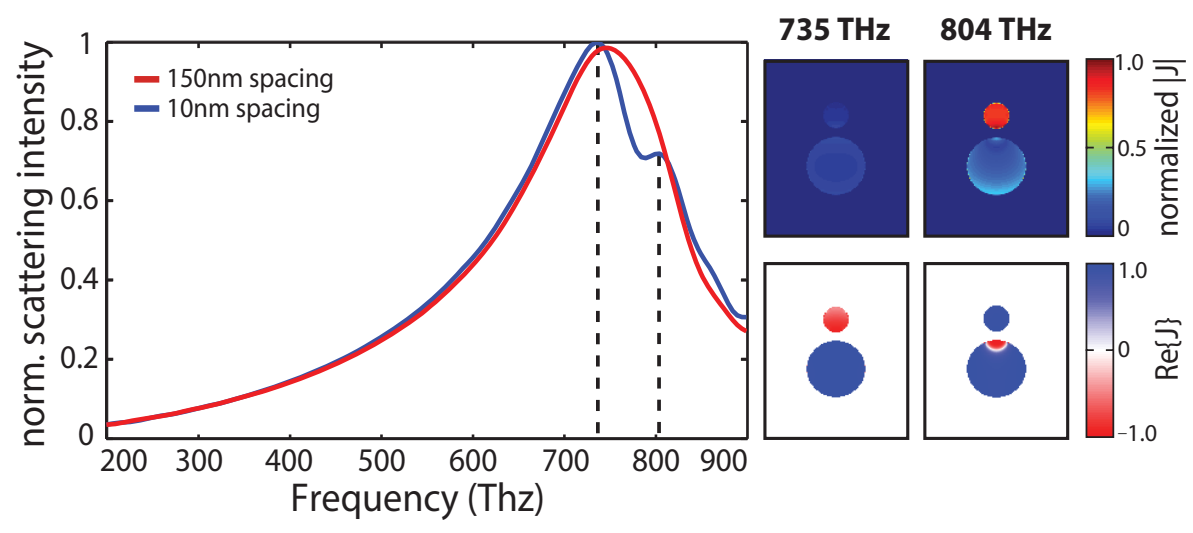

Figure 4.10: Two coupled sphere antennas. (Left) scattering intensity spectra of two coupled spheres with a spacing of 150 and $10 \mathrm{~nm}$. (Right) absolute and real current density plots at the two resonance frequencies, indicated with dashed lines. The symmetric and anti-symmetric nature of the coupled particles is revealed in the phase plot where the Real part of the current density is plotted.

The third investigated optical system shows the coupling of a dipole bar antenna with a higher-order mode, which is provided by a longer bar. The higher-order mode show a strong reduced polarizability. For the third optical systems the long bar is tuned so that it contains a second order bar mode. The scattering spectra of the coupled and uncoupled system is shown in Figure 4.11, revealing again a single peak in the uncoupled and two peaks in the coupled state. Also this system shows that the anti-symmetric eigenmode is strongly red-shifted while the symmetric eigenmode is almost stationary if coupling is increased. This time, however, due to the presence of a higher-order mode the direct relation between the polarizability and volume integral of the current density is no longer valid. In other words, the comparison of the cumulative current density of two antennas with different mode profiles does not yield any sensible information. The relation between volume and polarizability is still valid, in the sense that increasing the mode volume of a 
higher-order mode by a certain factor, linearly affects its polarizability. Although no absolute value of the polarizability ratio can be obtained from the current density plots, comparing the same mode profile and the resulting current density changes for the symmetric and anti-symmetric eigenmodes is still useful. Similar to the two-spheres system, the long bar in the coupled bar-bar system shows a strong overall current density increase for the symmetrical eigenmode of the coupled system. This indicates that the polarizability of the second order mode, having a lower initial polarizability, is enhanced.

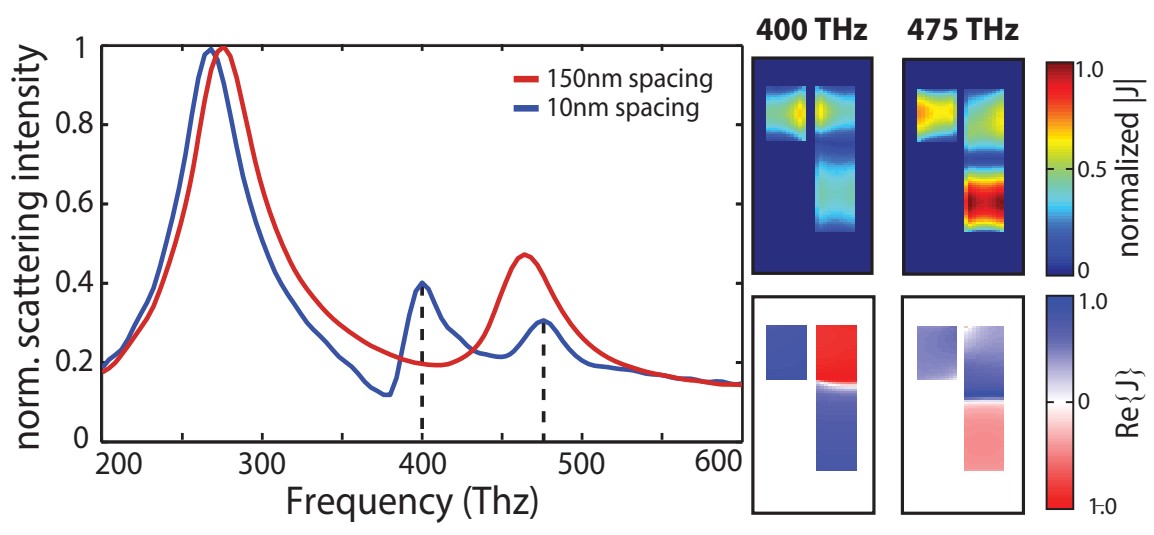

Figure 4.11: Coupling to higher order modes. (Left) scattering intensity spectra of two coupled bars, with a spacing of 150 and $10 \mathrm{~nm}$. (Right) absolute and real current density plots at the two resonance frequencies indicated with dashed lines. The symmetric and anti-symmetric nature of the coupled particles is revealed in the phase plot, where the Real part of the current density is plotted.

\subsection{Coupled Harmonic Oscillator model}

The resonant nature of localized plasmons makes them well suited for using analogous mechanical models to understand some of their basic behavior $[98,113,118,119]$. A successful example of this is the analogy with the mass spring oscillator model, which has been used by Zuloaga et al. to provide an intuitive explanation of the frequency shifts between near-field and far-field peaks [70]. In such a model the mechanical mass is analogous to the effective mass of the free electrons, and the spring constant is analogous to the electrostatic force on the charge distribution. Similarly, the kinetic and potential energy in a mechanical system are equivalent to the magnetic and electric energy, respectively. For ex- 
ample, when the kinetic energy is maximum, all the energy is located in the movement of free electrons, creating a strong current and a strong magnetic field. Also, a mechanical model based on coupled harmonic oscillators [120] has been applied to describe the interactions of two or more identical optical resonators and the new properties that arise, such as mode interference effects.

In this section a modified $\mathrm{CHO}$ model will be used to describe the polarizability ratios of the two antennas which make up the symmetric and anti-symmetric eigenmodes of a coupled two-antenna system. In particular we are interested in the coupling of two antennas showing different polarizability ratios. First, the translation from the mechanical to the optical is made, and a mechanical parameter for the optical polarizability of an antenna is introduced. In the last part of this section, the modified CHO model is used to describe the coupling of two nonidentical oscillators having different polarizabilites. With this model we are able to describe the spectral behavior of coupled two-antenna systems, showing an asymmetric splitting of the eigenmodes, and show that for the symmetric eigenmode the polarizability ratio of the two oscillators is equal to 1:1, regardless of their intrinsic polarizability ratio. The later, is of great importance in order to describe the angle dependent scattering patterns which have been shown in Figure 4.5.

\subsubsection{From mechanical to optical}

Building on what has already been done $[59,60]$, in this section the $\mathrm{CHO}$ model will be expanded so that also the coupling of two optical antennas with different individual polarizability and Q-factors can be described. This is an important step, as it is rarely the case that two identical optical resonators are coupled.

It is generally accepted that one can view the mass of, and the restoring force impinging on, the free electron cloud of an optical antenna as the mechanical equivalence of the mass $(m)$ and spring constant $(k)$. The mass of the electron cloud then simply scales with volume, however the restoring force that the free electrons encounter is strongly dependent on the shape of the antenna and the resulting electric field distribution. When making the step from the mechanical to optical, it is assumed that the coupled system is behaving completely linear, so that the coupled modes can be described as a linear combination of the individual antennas. The CHO model only describes how the amplitudes and phase of the individual oscillators change due to coupling. The optical equivalence of the coupling constant $\left(k_{c}\right)$ in the mechanical model, however, is 
not trivial to describe, as it is related to the mode overlap of the coupling antennas. However a sense of the magnitude of $k_{c}$ can be obtained by looking at the spectra of the coupled system, as the splitting of the eigenvalues is related to $k_{c}$.

Now we will describe how the movement of a mechanical oscillator relates to the polarizability of an optical resonator. In the optical equivalence, the driving force $(\mathrm{F})$ is replaced by a driving field $(F=-e E)$, where $E$ is a harmonically modulating field $E=E_{0} e^{-i \omega t}$ and $e$ the free electron charge. The total current inside an antenna $J_{P}$ at a given time can be expressed as a function of the velocity of the free electrons: $J_{p}=$ $-N e \frac{d x}{d t}=\frac{d p}{d t}$, with $N$ being the amount of electrons and $p$ the dipole moment. From the current $J_{p}$ expression the polarizability $\alpha$ is derived, which is found to inversely scale with the factor $\mathbf{R}_{p}$ :

$$
\begin{aligned}
p & =-N e x=\alpha E \\
\alpha & =\frac{N e^{2}}{\mathbf{R}_{p}\left(k-m \omega^{2}-i \mathbf{R}_{q} \omega \gamma\right)} \longrightarrow \alpha \propto \frac{1}{\mathbf{R}_{p}}
\end{aligned}
$$

Now there are two contributions to the polarizability $\alpha \propto \frac{1}{\mathbf{R}_{p}}$ and $\alpha \propto N e^{2}$. At first glance, both terms could highlight the volume scaling aspect of the polarizability of an optical antenna. The polarizability scales linearly with $N$, the number of electrons, and thus scales linearly with volume. Although the relation between volume and polarizability was shown to be generally valid [23], the reality is a bit more complicated. Comparing for instance the polarizability of a dipole antenna with an antenna which exhibits a higher order mode, it immediately becomes apparent that the polarizability is also dependent on the antenna shape. Because $N e^{2}$ is clearly associated to changes of volume, the view on the role of $\mathbf{R}_{p}$ should be revised, and seen more as a polarizability parameter which depends on the shape of an antenna. For the sake of convenience, we combine the two polarizability scaling parameters, $\mathbf{R}_{p}$ and $N e^{2}$ into a single parameter: $\mathbf{R}_{p}^{\prime}=\frac{\mathbf{R}_{p}}{N e^{2}}$, so that $\alpha \propto \frac{1}{R_{p}^{\prime}}$. In Appendix C.1 a mechanical oscillator model is given, which describes the mechanical force balance of a single (optical) resonator.

\subsubsection{Coupling of two loss-less mechanical oscillators}

Now we consider the coupling of two non-identical loss-less mechanical oscillators. The mass and spring constant of one of the oscillators scales with $\mathbf{R}_{p 1}=\mathbf{R}_{p}$ and for the other oscillator $\mathbf{R}_{p 2}=1$, so that the mass and spring ratio of the two oscillators scales with the factor $\mathbf{R}_{p 1,2}^{\prime}=$ $\mathbf{R}_{p} / 1$. Although the oscillators have different magnitudes for $\mathrm{m}$ and $\mathrm{k}$, 
both have the same resonance frequency: $\omega=\sqrt{\frac{\mathbf{R}_{p 1,2}^{\prime} k}{\mathbf{R}_{p 1,2}^{\prime} m}}=\sqrt{\frac{k}{m}}$. The eigenvalues and eigenvectors are derived by setting up the force balance of a two-resonator system with a coupling spring $k_{c}$ :

$$
\left(\begin{array}{cc}
m & 0 \\
0 & m \mathbf{R}_{p 1,2}^{\prime}
\end{array}\right)\left(\begin{array}{l}
\ddot{x}_{1}(t) \\
\ddot{x}_{2}(t)
\end{array}\right)+\left(\begin{array}{cc}
k-k_{c} & k_{c} \\
k_{c} & \mathbf{R}_{p 1,2}^{\prime} k-k_{c}
\end{array}\right)\left(\begin{array}{l}
x_{1}(t) \\
x_{2}(t)
\end{array}\right)=0
$$

We note that the sign of $k_{c}$ is inverted compared to its mechanical equivalence, as the coupling mechanism in the case of optical resonators is attractive, rather than repulsive in nature. The coupling matrix is chosen such that the two eigenmodes show an asymmetric splitting, which best fits the scattering behavior seen in Figures 4.4, 4.10 and 4.11. Using a test function: $x(t)=A \cos (\omega t-\phi)$, the force balance is rewritten to the form:

$$
\left(\begin{array}{cc}
\frac{k-k_{c}}{m}-\omega^{2} & \frac{k_{c}}{m} \\
\frac{k_{c}}{\mathbf{R}_{p 1,2}^{\prime} m} & \frac{\mathbf{R}_{p 1,2}^{\prime} k-k_{c}}{\mathbf{R}_{p 1,2}^{\prime} m}-\omega^{2}
\end{array}\right)\left(\begin{array}{l}
A_{1} \\
A_{2}
\end{array}\right)=0
$$

The eigenvalues and eigenvectors are then derived:

$$
\begin{array}{ll}
\omega_{1}=\sqrt{\frac{k}{m}} & \omega_{2}=\sqrt{\frac{k}{m}-\frac{k_{c}\left(1+\mathbf{R}_{p 1,2}^{\prime}\right)}{\mathbf{R}_{p 1,2}^{\prime} m}} \\
A_{1}=\left(\begin{array}{l}
1 \\
1
\end{array}\right) & A_{2}=\left(\begin{array}{c}
-\mathbf{R}_{p 1,2}^{\prime} \\
1
\end{array}\right)
\end{array}
$$

The eigenvalues show a single resonance, splitting into two resonances for increasing coupling. Interestingly, the first eigenmode of the coupled system, $\omega_{1}$, does not contain the coupling term $k_{c}$, and is positioned at the resonance frequency of an uncoupled oscillator. The second, antisymmetric eigenmode, however, is strongly dependent on $k_{c}$ and $\mathbf{R}_{p 1,2}^{\prime}$, and is strongly redshifted for increasing $k_{c}$ and $\mathbf{R}_{p 1,2}^{\prime}$. The amplitudes of the individual oscillators for the two eigenmodes of the coupled system are found by calculating the eigenvectors. The eigenvectors $\left(A_{1}\right)$ and $\left(A_{2}\right)$ in Equation 4.4 show that the amplitudes of the first and second eigenmodes of the coupled system are symmetric and anti-symmetric in nature, respectively. The derived eigenvectors show very interesting behavior due to the introduction of $\mathbf{R}_{p 1,2}^{\prime}$. The amplitude of the two individual oscillators of the anti-symmetric eigenmode scale with $\mathbf{R}_{p 1,2}^{\prime}$, 
which is exactly the same as the amplitude ratio of the antennas when they are in the uncoupled state. However, the eigenvalues/amplitude of the individual oscillators of the symmetric eigenmode are equal, even though the respective magnitudes of their $m$ and $k$ differ by a factor $\mathbf{R}_{p 1,2}^{\prime}$. This is an interesting result, as this shows that oscillators with a low intrinsic amplitude/polarizability could be enhanced, by coupling with an oscillator which has a higher intrinsic amplitude/polarizability.

Appendix C.2 describes the effect of loss and thus the Q-factor ratio of a coupled two-antenna system. It is shown that loss does not influence polarizability ratio of a coupled system and again a 1:1 polarizability ratio is found for the antennas which form the symmetric eigenmode, regardless of the polarizability ratio of the fundamental antennas.

\subsection{Conclusions}

We have reported on the coupling of two non-identical antennas which have different polarizabilities. The spectral behavior and angle dependent scattering patterns of a coupled and un-coupled bar-ring antenna system has been investigated, revealing that for the symmetric eigenmode of the coupled system, the polarizability ratio of the two antennas is of equal magnitude. This interesting result is corroborated by two other two-antenna systems which show the same enhancement of the antenna having the low intrinsic polarizability, in the case of the symmetric eigenmode. With a modified coupled harmonic oscillator model, the coupling of two antennas with different polarizabilities are analytically described, which indeed shows that for the symmetric eigenmode the polarizability ratio of the two oscillators is equal to $1: 1$, regardless of their intrinsic polarizability ratio. This result opens a new path in designing/manipulating the angle dependent scattering patterns of coupled antenna systems. For many optical applications where directional control on the emission is needed, which is the case in photovoltaic and LED lighting applications, this new design path could be usefull. 


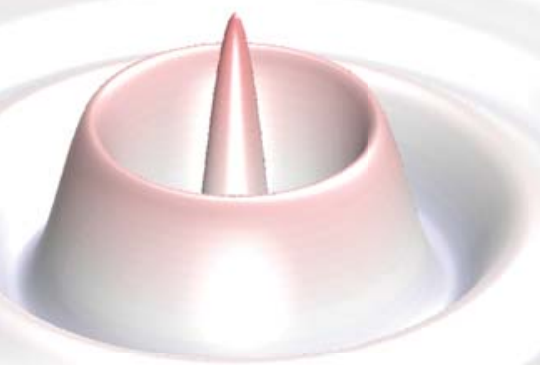




\section{Part II}

Antennas for Plasmons 

Chapter

\section{Investigation of single hole-antennas}

Holes in metallic films have been the subject of theoretical and experimental study for over several decades. Their capability of generating metal-dielectric surface waves, which are strongly localized to the interface, is what makes hole-structures interesting for many optical applications. In the second part of this thesis, the central aim is the manipulation of the surface waves, which are present on a metal-dielectric interface, using arrays of hole-antennas. This chapter provides a study on the basic elements which are needed in that regard. First we explore how the transmission through a hole-antenna is affected when the hole diameter is reduced to the point of cutoff, where the propagation constant becomes imaginary. The dispersion of light, which is associated with the transmission of light through sub-wavelength holes near cutoff, is experimentally and numerically investigated. In the second part of this Chapter we numerically show the field distributions of subwavelength holes made in gold (Au) and aluminum ( $\mathrm{Al}$ ) substrates and demonstrate how the shape of the hole effects its field distribution. In the third part of this Chapter we use a near-field microscope to investigate the EM near-fields of holes with various hole diameters. The near-field signals are found to contain both the in-plane electric and magnetic field components. Further, we investigate how the ratio of the EM field components depend on the diameter of the holes.

\subsection{Introduction}

In this Chapter, we discuss the optics of single sub-wavelength holes. Their transmission [121-123], near-field distribution [124-128] and abil- 
ity to launch SPPs are relevant in two quite distinct ways in this thesis. First of all we are interested in generating (and in Chapter 6 controlling) SPPs. However, the near-field aperture we use to measure, is also a hole in a metal film. To distinguish both we use 'hole' when discussing the hole in a continuous film, and 'aperture' if we want to indicate the hole in the NSOM probe (see Figure 5.1). The former is in this thesis always in $\mathrm{Au}$, while the latter is in Al. Since the physics of holes and apertures is very similar, many of the things we show is valid for both, though the differences in dielectric constant of the materials can lead to clear differences. In the first section of this Chapter we numerically and experimentally investigate holes in a thick $(3.5 \mu \mathrm{m})$ gold film, having diameters close to cutoff. Using the hole diameter to realize different phase delays, we discuss the manipulation of SPPs using phased hole arrays. In the second part of this Chapter, we show how the near-field distribution of a single hole is affected by the shape of the hole, by numerically investigating three basic shapes. The difference in near-field distribution between holes surrounded by a gold or aluminum film is discussed. This is relevant as in the NSOM experiment, the field distribution of the NSOM probe plays a major role in its selectivity and determines which field components are detected by the NSOM, as will be discussed in Section 5.4. It is therefore very important to understand which parameters affect the field distribution of these sub-wavelength holes.

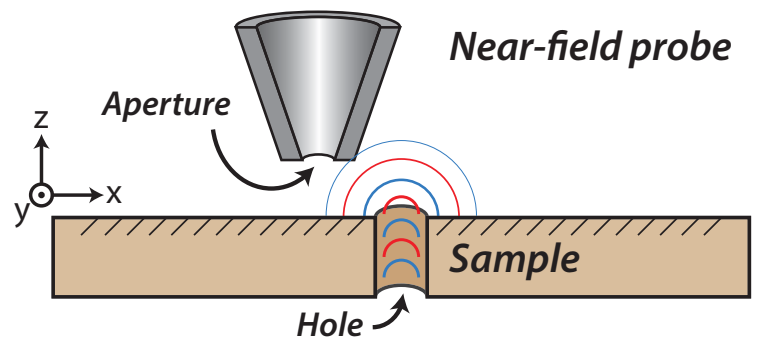

Figure 5.1: Cartoon depicting the NSOM configuration. Cartoon depicting the near-field probe in relation to the sample surface.

In the third and final section of this Chapter we further discuss the different field components we measure in our NSOM experiments. Feber et al. have shown, in near-field measurements on photonic crystal waveguides, that ordinary aperture near-field probes are sensitive for both the electric and magnetic field in-plane field components [129]. The sensitivity of the near-field probe for both EM in-plane field components can be 
understood by the reciprocity theorem, which takes EM field distribution of the near-field probe into account (for a detailed explanation see Appendix D). In order to correctly interpret the NSOM measurements on multiple holes, we investigate the near-field distributions of various single holes with different hole diameters, made in a $250 \mathrm{~nm}$ thick gold substrate, and determine whether our experimental data also contains both electric and magnetic field components.

Isolated circular holes can be used as local sources of SPPs [130-133] and coupling efficiencies up to $20 \%$ have been realized [133]. We will use this property of sub-wavelength holes in metallic films in Chapter 6 to steer the direction of propagation of SPPs.

\subsection{Transmission through sub-wavelength holes}

The transmission through a subwavelength hole is generally described as the transmission through a waveguide [134]. The waveguide modes for a square hole shape can be analytically described for a perfect conductor, [135]. Holes in real metals such as aluminum and gold can not be described as a perfect conductor and the relation between the cutoff wavelength and hole dimension is more complex. However it is instructive to consider this situation as it clearly shows the relation between the cutoff wavelength and the dimensions of the hole. The propagation constant $(\beta)$ is described as:

$$
\begin{aligned}
\beta & =\sqrt{(w / c)^{2}-\left[\beta_{x}^{2}+\beta_{y}^{2}\right]} \\
& =\sqrt{(w / c)^{2}-\pi^{2}\left[(m / \alpha)^{2}+(n / \alpha)^{2}\right]}
\end{aligned}
$$

where $\beta_{x}$ and $\beta_{y}$ are the lateral propagation constants, which are orthogonal to the direction of propagation, $(m, n)$ the waveguide modes, and $(\alpha)$ describes the lateral dimensions of the square hole. The cutoff wavelength $\left(\lambda_{c}\right)$ for the lowest order mode $\left(T E_{10}\right)$ of a square hole is found to scale with the width $(\alpha)$ of the hole: $\lambda_{c}=\alpha / \pi$. For $\lambda<\lambda_{c}$ the propagation constant $\beta$ is real and the waveguide modes in the hole can propagate, with the amplitude of the form $A_{x}=e^{i x \beta_{x}}$. For wavelengths larger than the cutoff wavelength, $\beta$ is imaginary and the transmission is exponentially damped. In real metals the fields can penetrate into the metal. For $\mathrm{Au}$ this is $\approx 24 \mathrm{~nm}$ and this has a significant effect on the cutoff wavelength, which as a result is redshifted [136]. As can be seen in Equation 5.1, in cutoff, the propagation constant vanishes and light which is propagating through the hole, has no phase delay. In real metals the propagation constant retains a small real part and the cutoff 
wavelengths become very large $[137,138]$. A zero- $\lambda$ Fabry-Perot resonance is formed in the leaky cavity, which is formed by the reflection at the outer ends of the hole. These resonances are often called localized resonances as they are localized in the hole and therefore show no dispersion.
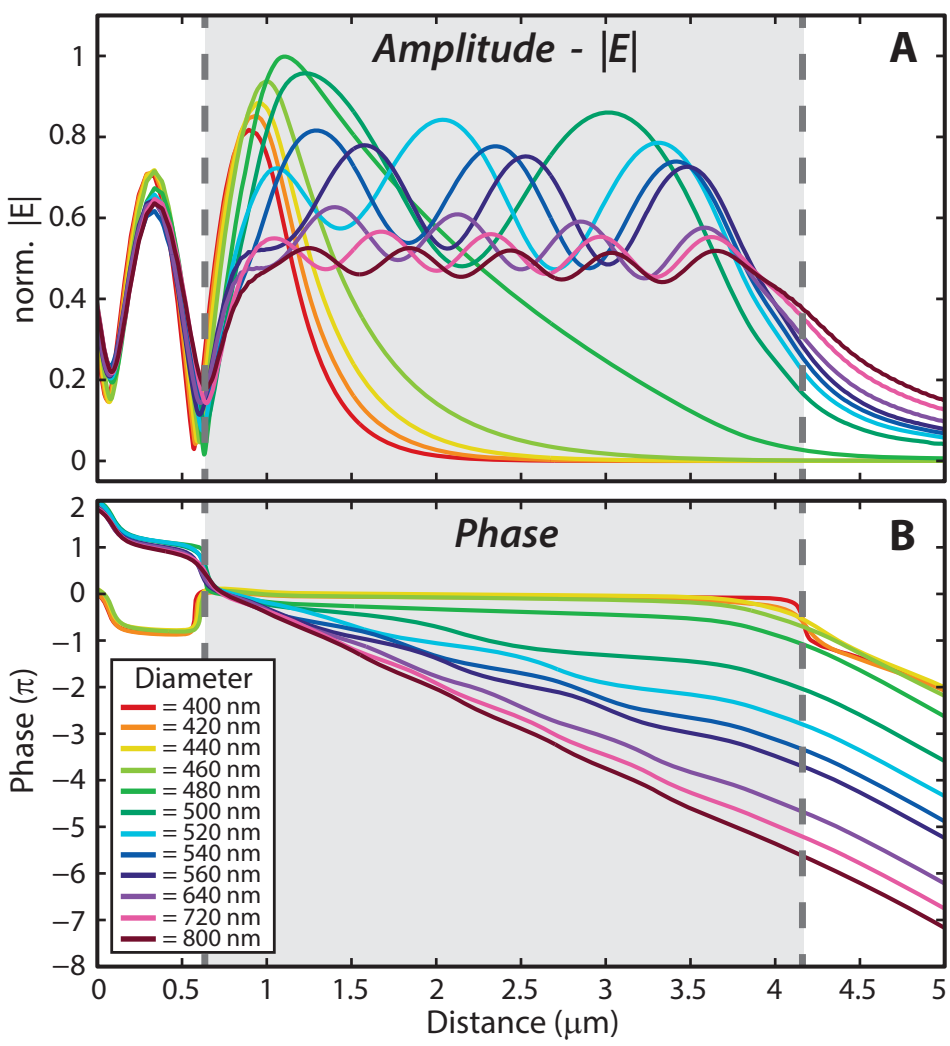

Figure 5.2: Amplitude and accumulated phase delay of light passing through holes of various diameters. Numerical calculations, showing the amplitude (A) and phase (B) of EM waves, propagating through holes of various diameters in a gold substrate. On the horizontal axis the propagated distance orthogonal to the surface is shown. The gray and white area indicate the position of the hole/substrate and air, respectively. Polarized light with a wavelength of $950 \mathrm{~nm}$ is used.

To illustrate the transition from a guiding waveguide mode to an exponentially damped cutoff mode, Figure 5.2 shows numerical calculations (FID, [139]) of the amplitude (A) and phase (B) of light (950 nm), which is propagating through a circular holes having various radii. The holes are made in a film of 3.5 micron thick gold, indicated with the gray area. 
The amplitude graph shows that a standing wave pattern is formed before light passes through the hole. This standing wave is formed due to the interference of the incoming beam and the reflection of the interface, which is marginally affected by the hole size. Also inside the hole itself, standing waves are observed for holes with a diameter of $500 \mathrm{~nm}$ and larger. The amplitude of the standing wave increases with reducing hole diameter, to the point that the standing wave disappears and only an exponential decay is observed, indicating that the diameter has become smaller than the cutoff wavelength. Examining the standing wave pattern, the increase of the wavelength with decreasing hole diameter is clearly visible. The increase of the wavelength can also be observed in the phase, which is plotted in Figure 5.2 B. Following the curve of a hole with a diameter of $800 \mathrm{~nm}$, one observes that the slope of the curve in the hole and after it has passed through is roughly equal, showing that the hole has almost no effect on the phase. However, upon reducing the hole diameter the slope in the hole becomes significantly more shallow, as has been described [137,138]. After light has passed through, the phase slope again becomes equal, independent of hole size. This means that by varying the diameter of the hole and using a film thickness of $3.5 \mu \mathrm{m}$, a phase delay of almost $6 \pi$ can be achieved.

The effect of hole diameter (cutoff) on the dispersion of propagated light is experimentally investigated, by illuminating two holes with different diameters in a $3.5 \mu \mathrm{m}$ thick $\mathrm{Au}$ film. The resulting field distribution is examined using a near-field microscope see Figure 5.3. The real component of the field distribution is shown in panel $\mathrm{B}$, and a cross section of the amplitude and phase, taken above the two holes and indicated with the green line, is plotted in C. Due to the extreme aspect ratio of the hole dimensions the designed cylindrical hole ends up being conical in shape (see Figure 5.3 D), which is assigned to strong redeposition during fabrication (see appendix A.3). To counteract this effect, larger holes are made with an average hole diameter of 480 and $690 \mathrm{~nm}$, resulting in a reduced overall phase delay, which can be obtained. By taking the fabrication artifacts, which lead to vertical tapering of the holes, into account in our numerical calculations, a phase difference of $\pi$ was expected, however the cross section in $\mathrm{C}$ reveals a smaller phase shift of only $\pm 0.3 \pi$. Because the size of the aperture of the NSOM probe is sufficiently smaller than the size of the two holes, the resolution of the NSOM measurements should be high enough, and no smoothing occurs which would reduce the observed phase. The measured amplitude ratio above the holes is approximately 2 and this is smaller compared to 


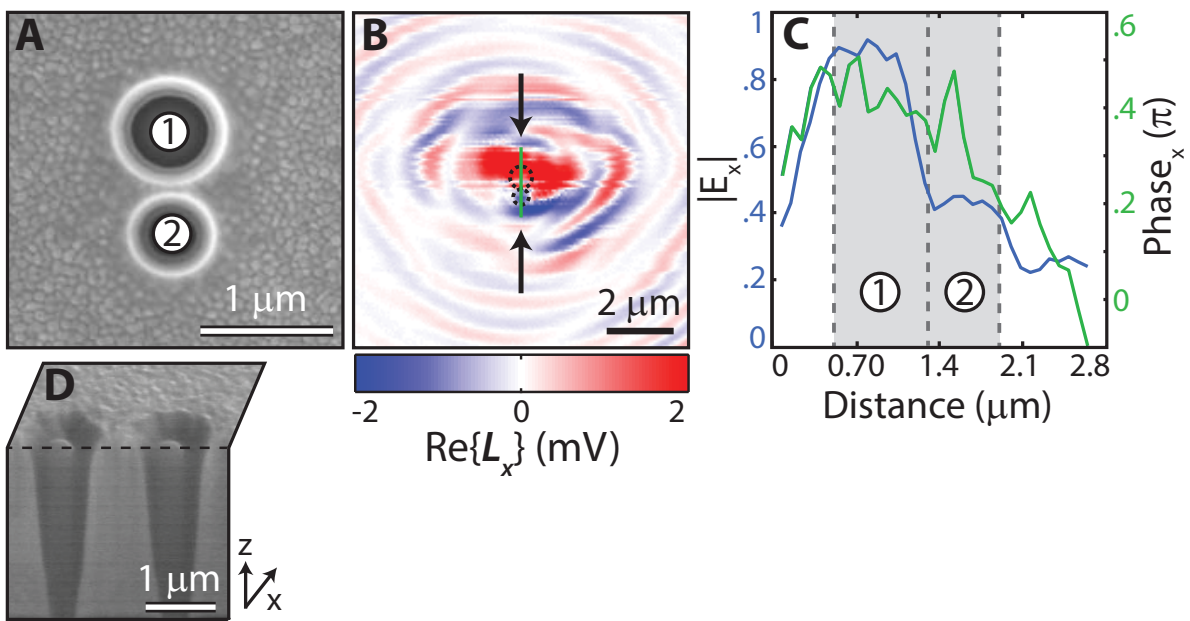

Figure 5.3: Measuring phase delay of a two-hole system. The phase delay of two holes with an average diameter of 480 and $690 \mathrm{~nm}$, in a gold film of 3.5 $\mu \mathrm{m}$ thick, (SEM image shown in $(\mathbf{A})$ ), is measured with a near-field microscope. The real component of the field distribution is shown (B) and a cross section, indicated with the green line, is plotted $(\mathbf{C})$ where the amplitude and phase of $L_{x}$ are plotted. The numbered / dotted circles in (A) and (B) and the gray area in $C$, indicate the position of the two holes. (D) shows a SEM micrograph of a vertical cross cut through the gold substrate, showing the vertical profiles of the fabricated holes.

what what we expect from the numerical calculations. The discrepancy is tentatively attributed to an extra path for light to get in the nearfield probe; this on its own is probably due to a hole in the Al coating. The low periodicity $(>\lambda)$ that we observe in Figure $5.3 \mathrm{~B}$, could be introduced by a hole in the coating of the near-field probe. If the fields from this coating artifact are of significant amplitude and $0.5 \pi$ out of phase with the fields collected by the aperture of the probe, a reduction
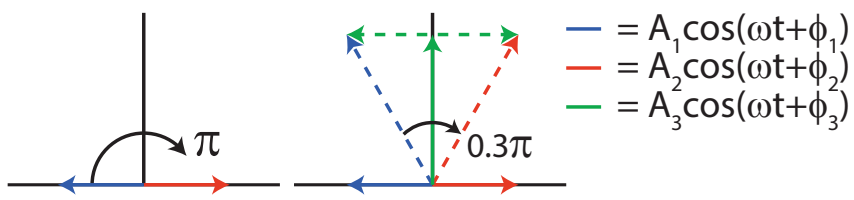

Figure 5.4: Cartoon showing the effects of a leaking near-field probe. Cartoon showing how the measured field components, and in particular measured phase can deviate from the local fields (left) when an extra field component, which is $0.5 \pi$ out of phase, is added (right). 
of the phase difference between the holes would then be observed (see Figure 5.4). This would also explain the reduced amplitude ratio above the holes. The magnitude of the measured phase reduction depends on the amplitude and phase difference of the two sources. Using Fourier filtering techniques low periodic signals can be suppressed, which led to our conclusion that the aperture coating was leaking and a thicker aluminum coating was needed. In the near-field measurement shown in the rest of this thesis a near-field coating of 250 instead of $150 \mathrm{~nm}$ was used, after which the artifacts were no longer observed.

Figure 5.5 depict a possible SPP beam shaping structure, based on the phase delay associated with a series of holes with decreasing diameters around cutoff. The structure consists of five holes with decreasing hole diameters, leading to an increasing phase delay resulting in an SPP beam which is propagating under an angle. In order to design arbitrary phased hole arrays, a full experimental characterization of the diameterdependent phase delay has to be made. However the fabrication of these structures is not so easy, due to the aspect-ratio of the hole, leading to tapering effects. We found that tuning the phase can only be done in a small diameter window of about $150 \mathrm{~nm}$. Therefore we abandoned this strategy and instead went for an approach which does not rely on fabricated structures to obtain a certain phase delay, which we discuss in Chapter 6.
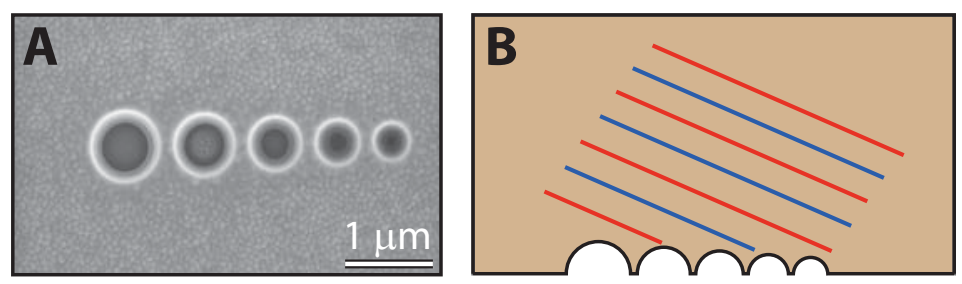

Figure 5.5: Shaping the propagation direction of SPPs. (A) a SEM micrograph and (B) cartoon depicting an SPP beam shaping structure, consisting out of five holes with decreasing hole diameter. The red and blue line in $B$ indicate the propagation of SPPs.

\subsection{Near-field distribution}

In this thesis, we study light emerging from holes in Au being picked up in the near-field by a aperture in Al, the near-field probe. It is therefore very important to study the near-field distribution near holes, and investigate how the material surrounding the hole and the shape of the hole 
affect the near-field distribution. The electromagnetic fields emerging from a circular hole has been experimentally investigated by scanning an aperture probe of a near-field microscope over molecules [140-144]. Figure 5.6 shows numerical calculations of the EM field distributions of a single hole with a diameter of 300 and $225 \mathrm{~nm}$, made in a $250 \mathrm{~nm}$ thick gold (above) and aluminum (below) substrate, at $10 \mathrm{~nm}$ height above the substrate surface. The holes are illuminated with horizontally polarized light (indicated with the black arrow), having the direction of propagation orthogonal to the sample surface. The diameter of the holes in the $\mathrm{Au}$ and $\mathrm{Al}$ substrates are chosen such that they best represent the experimental conditions. Both numerical calculations of the field distributions of holes in $\mathrm{Au}$ and $\mathrm{Al}$ substrates show a great resemblance to the field distributions, which can be calculated using Bethe and Boukamp formulations, however in these numerical calculations also the excitation of propagating surface plasmon polaritons (SPP) are taken into account. The calculations by Bethe and Boukamp also show a Gaussian-like and four-lobed anti-phased distribution for the $E_{x}, H_{y}$ and $E_{y}, H_{x}$ field components, respectively. Note that the magnetic field components are approximately 300 times smaller than those of electric fields. This is also known as the wave impedance: $Z=|E| /|H|=\sqrt{\mu / \epsilon} \approx 1 / 120 \pi$.

\subsubsection{Substrate material}

Although the field distributions of the holes in $\mathrm{Au}$ and in $\mathrm{Al}$ are very similar, when comparing them in detail two striking differences emerge. Firstly, the holes made in $\mathrm{Al}$ show a stronger localization that can be explained by the fact that the diameter of the hole is smaller [145]. However, more importantly, the real part of the dielectric constant $(\operatorname{Re}\{|\epsilon|\})$ of $\mathrm{Al}$ is larger compared to $\mathrm{Au}$, meaning that the skin depth in $\mathrm{Al}$ is smaller and the field distribution is more localized on the metal-dielectric interface [145]. Secondly, the ratio of the $E_{x, y}$ is larger in the case of $\mathrm{Al}$ compared to holes made in Au substrate. This ratio difference may seem irrelevant, however the opposite is true: the signals which are measured using the $\mathrm{Al}$ coated near-field probe depend strongly on the ratio between $E_{x, y}$ (see Appendix D). If $E_{x, y}$ of the near-field probe are of almost equal value, it becomes impossible to disentangle the measured signal in terms of $H_{x}$ and $H_{y}$ (see Equation D.2). Choosing different kinds of materials to coat the near-field probe, which favor certain electric or magnetic field components, would offer a method to selectively measure certain EM fields and suppressing others. 


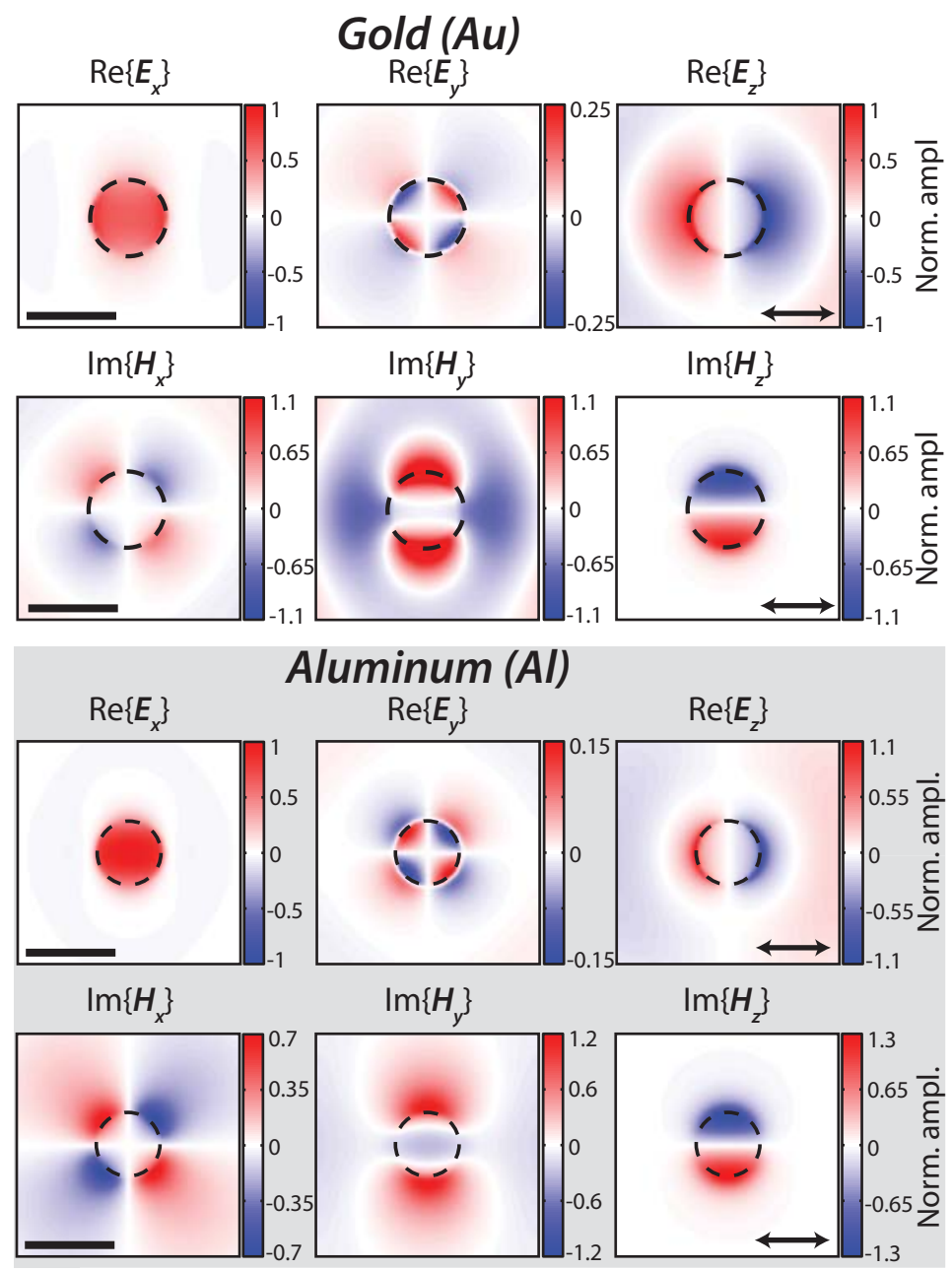

Figure 5.6: Electric and magnetic field distribution of a single hole. Numerical calculations of the EM field distribution, $10 \mathrm{~nm}$ above the surface of a single hole with a diameter of $300 \mathrm{~nm}$ and $225 \mathrm{~nm}$, made in Au (above) and Al (below) substrate. The hole is excited with horizontally polarized light (with $\lambda=950 \mathrm{~nm}$ ), indicated with the black arrow. The amplitudes are normalized to the maximum value of $E_{x}$; note that the amplitudes of the magnetic field distributions are multiplied by $10^{-3}$. The black bar indicates a length of $400 \mathrm{~nm}$.

\subsubsection{Hole shape}

It is not our intention to fully explore how the shape of a hole affects its optical mode in the resulting field distribution. However, as later in this section will become clear it is of vital importance to know the field distributions of studied holes and the aperture, used in the NSOM probe, to understand the experimental NSOM signals. With that in mind we limit 
our focus to the field distributions of three basic hole shapes: circular, square and rectangular. Over the years, the effect of hole shape on the SPP resonance has widely been investigated [134,146-149]. Figure 5.7 shows the effects of hole shape on the electric field distributions of three holes with a circular, square and triangular shape, made in a $250 \mathrm{~nm}$ thick gold substrate, shown at $10 \mathrm{~nm}$ height above the substrate surface. The holes are illuminated with horizontally polarized light (indicated with the black arrow), having the direction of propagation orthogonal to the sample surface. The electric field distributions are normalized to the $E_{x}$ of the circular hole. The field distributions of the square and

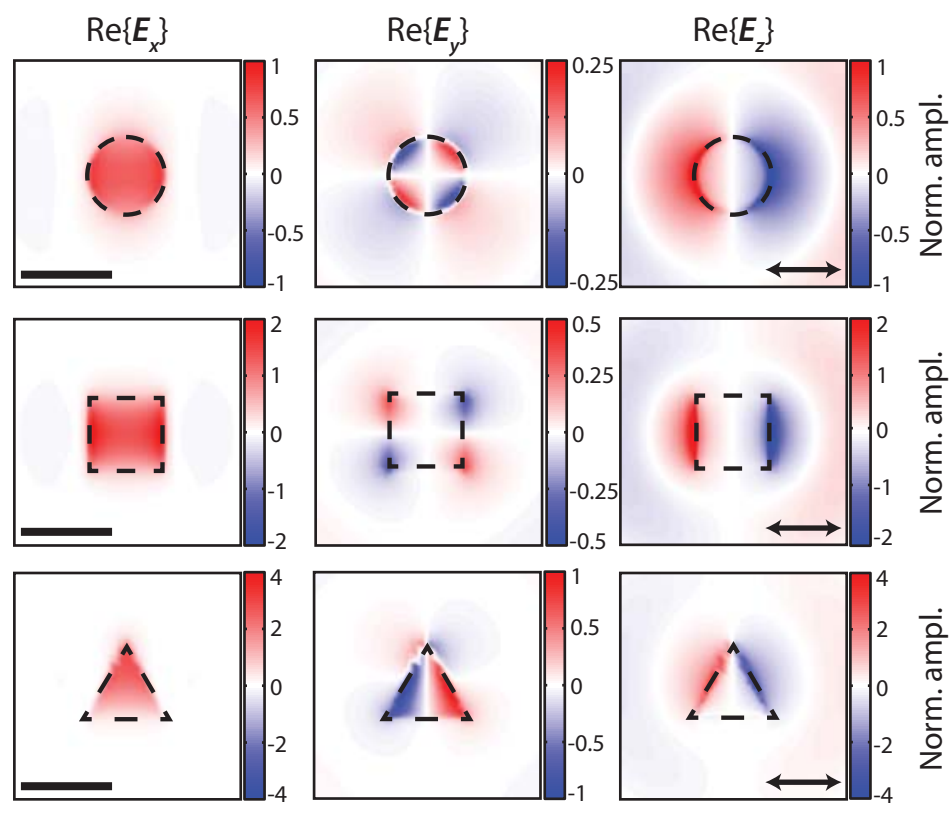

Figure 5.7: Electric field distribution of a single hole of different shapes. Showing numerical calculations of the real part of the electric field distribution, $10 \mathrm{~nm}$ above the surface of a circular, square and triangular hole, made in a 250 $\mathrm{nm}$ thick Au film, excited with horizontally polarized light (with $\lambda=950 \mathrm{~nm}$ ), indicated with the black arrow. The amplitudes are normalized to the maximum value of the $E_{x}$ of the circular hole. The black bar indicates a length of $400 \mathrm{~nm}$.

circular holes show great similarities, however on closer inspection the $E_{z}$ field components in the square hole seem to be more localized on the interfaces which are perpendicular to polarization of the driving field. Many studies have investigated the gradual change from a square to a rectangular hole shape, and two polarization dependent resonances have been observed [147], which are associated with the collective resonance 
of the two opposing interfaces. The circular hole, due to its symmetry, only shows one resonance and no polarization angle dependence. The electric field distribution of the triangular hole, and in particular the $E_{y}$ and $E_{z}$ field components, are quite different compared to those of the circular and square holes. Again from the reciprocity point of view, the $E_{y}$ component of the triangular shape is especially interesting. The field components which dominate the measured signal, according to the reciprocity theorem strongly depend on the shape and magnitude of the EM field components aperture probe. Breaking a degree of symmetry, as in the case of the triangular shaped holes, can lead to strong changes in the field distributions of certain EM components. If one could make a near-field probe with a shape that favors a certain EM component, it would again be possible to selectively measure certain EM components while suppressing others.

The strong field accumulates at the position where the two tapering interfaces of the triangle meet, showing an enhancement in the order of four times compared to the field distribution of the circular hole. Studies on the second harmonic generation (SHG) of triangular vs rectangular shaped holes revealed an astonishing 1-2 orders of magnitude increase in the generated SHG intensity for the triangular shaped holes [150]. The second harmonic generation shows a strong dependence on relatively small changes in geometry, such as its aspect ratio.

\subsection{Near-field measurement of a single hole}

Though in the early days of near-field optics the emphasis was on the ability to observe the electric field components of EM waves, recently it has been shown by Feber et al. that not only the in-plane electric, but also magnetic fields are measured using an aperture near-field microscope [129], so that the experimental signals are expressed as:

$$
L_{(x, y)}=\eta_{e} E_{(x, y)}+\eta_{h} H_{(y, x)} .
$$

where $\eta_{e}$ and $\eta_{h}$ describe the electric and magnetic sensitivity of an aperture probe (see appendix D). In the rest of this thesis, only the inplane electric and magnetic field components are considered, see Equation 5.2, when referring to the experimental data. From measurements on photonic crystal waveguides, using near-field probes with diameters ranging from 120 to $350 \mathrm{~nm}$, a roughly equal sensitivity to the electric and the magnetic in-plane field components was found, regardless of the aperture diameter [151]. In this section, we investigate the near-field 
distributions of holes in a gold film, which are illuminated with a plane wave, with a propagation direction orthogonal to the sample surface. We measure the ratios of the in-plane electric and magnetic field components for holes with varying hole diameters, which we compare with the results shown in [129]. We will explore the effect of cutoff on the measured electric and magnetic field strength of holes with diameters above and beneath cutoff. The ratio of free-space emission and SPP excitation is studied for different hole diameters. During this set of measurements, a single near-field probe is used, ensuring that only the effect of hole diameter in the $\mathrm{Au}$ layer is captured.

\subsubsection{Near-field measurement of a single hole}

A near-field microscope setup, see Figure 2.6, is used to obtain the field distribution of a single hole with a diameter of $300 \mathrm{~nm}$, where the parallel and orthogonal polarization components are detected (see Figure 5.8). The hole is illuminated with horizontally polarized light, indicated in
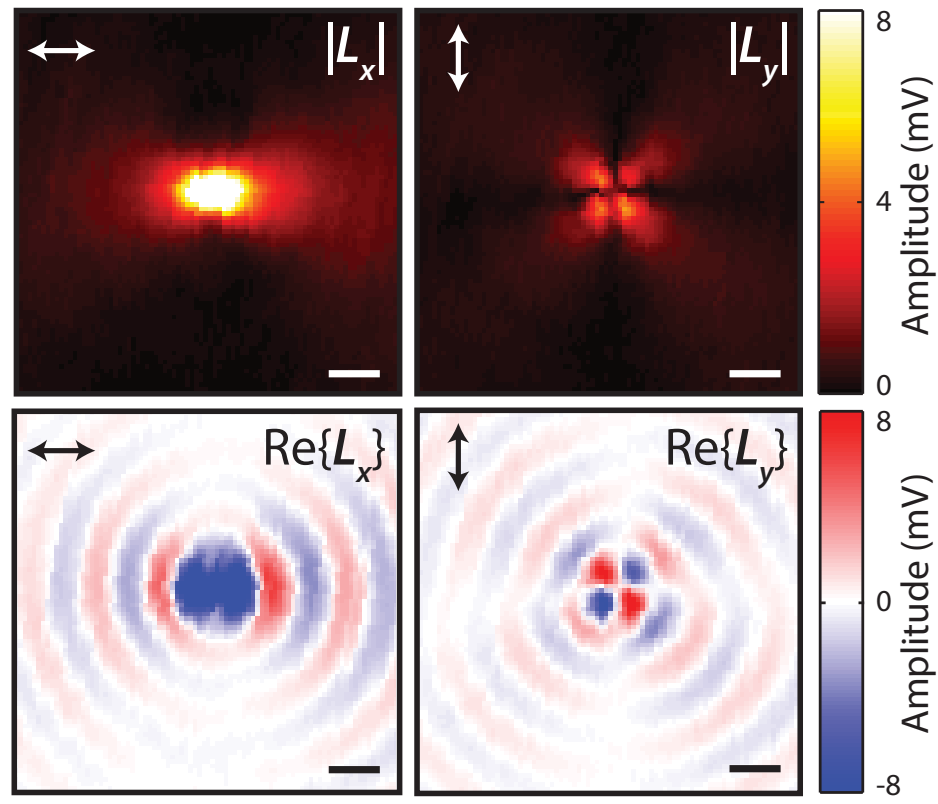

Figure 5.8: Experimental field distribution of a single hole-antenna. Parallel and orthogonal polarization components (indicated by the black and white arrows) of the field distribution of a single hole, with a diameter of $300 \mathrm{~nm}$. Above and below show the absolute and real part, respectively, of the field distribution. The black and white bars indicate a length of $500 \mathrm{~nm}$. 
Figure 5.8 with black and white arrows. A half- and a quarter-wave plate in the reference arm of the experimental setup are set such that the two experimental signals are linearly polarized and coincide with the vertical and horizontal polarization axis (for the alignment procedure see Section 2.2.2). When the wave plates are set correctly, the two obtained experimental signals closely resemble the $E_{x}, H_{y}$ and $E_{y}, H_{x}$ field distributions in Figure 5.6, showing a Gaussian-like and anti-phased four-lobed field distribution, respectively. In our experiments, the plasmonic nature of this mode is highlighted in the perpendicular polarization channel $\left(L_{y}\right)$, which at a distance of several micrometers from the sample surface, shows a vanishing signal, and only close to the sample surface is the four-lobed field distribution observed.

Because the near-field microscope is incorporated in a Mach Zehnder interferometer, we measure both amplitude and phase, making it possible to perform a two-dimensional Fourier transform of the field distribution. The reciprocal space or k-space shows the distribution of spatial frequencies that are present in the field distribution of a single hole. To demonstrate the relation between the distribution in k-space and the field distribution in real-space, we show an example where two different filters are applied on the k-vector distribution of numerically calculated $H_{y}$ (see Figure 5.9). The first row in Figure 5.9 shows the k-vector and real-space distribution of $H_{y}$ when no filter is applied. The second and third rows, respectively show how the field distribution in the real-space is affected by applying a disk and an inverted disk filter in $\mathrm{k}$-space. When a disk filter is applied in $\mathrm{k}$-space and the $\mathrm{k}$-vectors smaller than $2 \pi / \lambda$ are filtered out, two localized peaks remain. The real-space field distribution of the disk filtered signal shows a strong resemblance to the unfiltered field distribution, however at the position of the hole the field strength is strongly reduced. So it becomes clear that the broad field distribution in real-space, which is associated with propagating surface plasmons (SPP), is strongly localized in the two semi-circular lobes in $\mathrm{k}$-space. The sign in k-space indicates the direction of propagation [152]. The inverted disk filter only retains low k-vector components resulting in a real-space field distribution, which is dominated by a strong field localized at the position of the hole. The low k-vector components are associated with the free-space emission of the hole. 


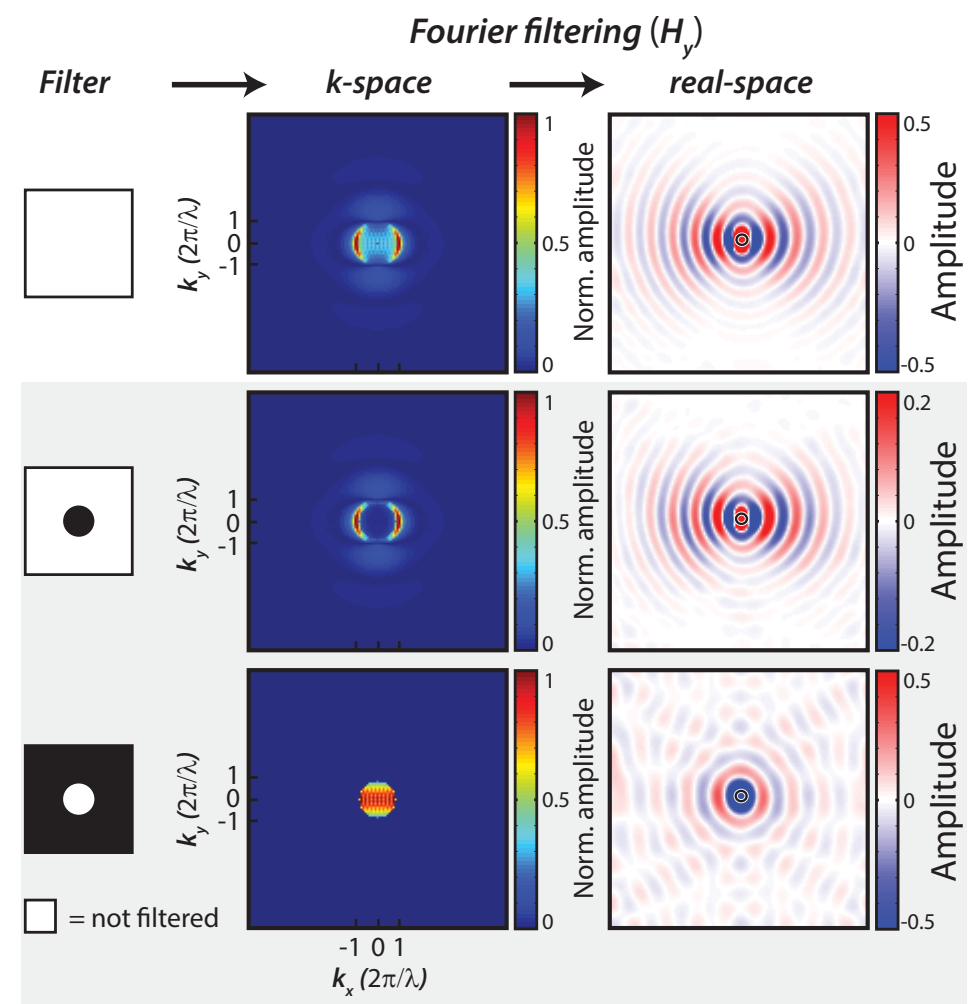

Figure 5.9: Fourier filtering on the numerically calculated $H_{y}$ field component of a single hole. Shows how the calculated field distribution in the real-space is affected by applying a disk (center) and an inverted disk filter (lower) in the k-space. The position of the hole is indicated with a black ring.

\subsubsection{K-space distribution of $E_{x, y}$ and $H_{x, y}$}

Figure 5.10 shows the two dimensional Fourier transform of numerically calculated $E_{(x, y)}$ and $H_{(x, y)}$ field components of a single hole, with a $300 \mathrm{~nm}$ diameter in a $250 \mathrm{~nm}$ thick $\mathrm{Au}$ film. In order to describe the measured near-field signals $\left(L_{(x, y)}\right)$ in terms of an electric and magnetic field component, we investigate the two dimensional Fourier transforms of numerically calculated in-plane EM fields. The k-vector distributions of the $E_{x}$ and $H_{y}$ field components both show the two peaks associated with the presence of SPP, indicated with white arrows. For low k-vector values $\left(|k|_{(x, y)}<1\right)$, the $\mathrm{k}$-vector distribution of $E_{x}$ is very different from $H_{y}$. Where $H_{y}$ shows a strong contrast between the SPP localization and the surrounding k-vectors, $E_{x}$ shows almost a flat plateau for $\left(|k|_{(x, y)}<\right.$ 1). This shows that $H_{y}$ is predominantly present in SPPs rather than free-space light, and in sharp contrast $E_{x}$ is strongly present in SPPs 

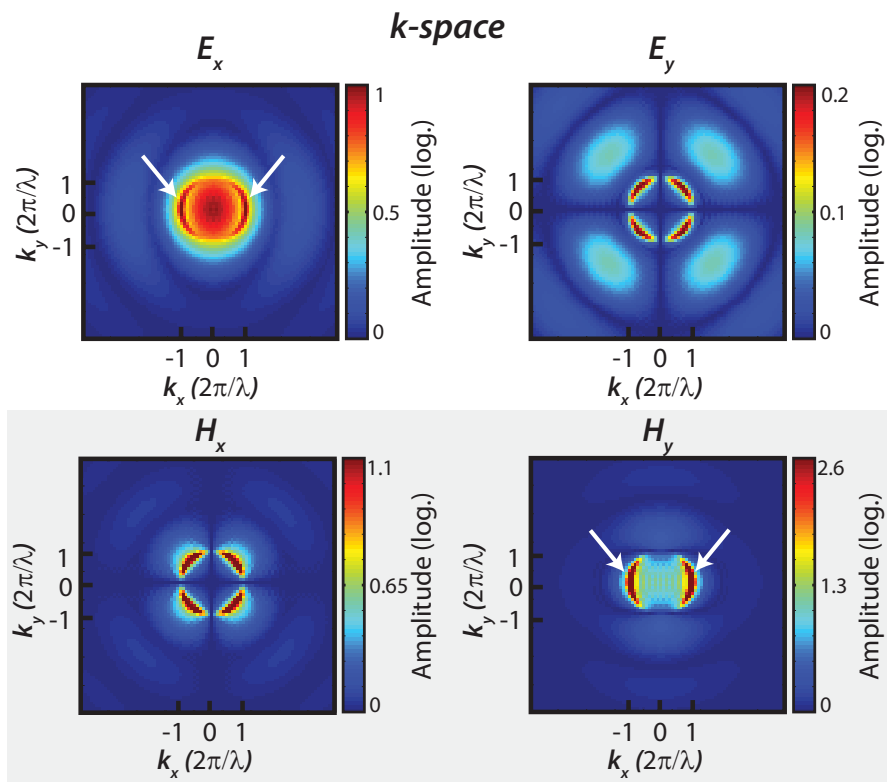

Figure 5.10: Two dimensional Fourier transform of the in-plane field components of a single hole. Showing the k-vector distribution of the numerically calculated $E_{(x, y)}$ and $H_{(x, y)}$ field components of a single hole with a $300 \mathrm{~nm}$ diameter in a $250 \mathrm{~nm}$ thick Au film, at a $10 \mathrm{~nm}$ height above the substrate. Note that the amplitudes are plotted on a log scale. The white arrows indicate the presence of SPP mode.

and free-space light, resulting in a flat plateau in k-space. It therefore is feasible to use the numerically calculated field distributions of $E_{x}$ and $H_{y}$ as a fitting parameter, to describe $L_{x}$. Similarly, the k-vector distribution of the $E_{y}$ and $H_{x}$ field components are investigated, however we find that the k-vector distributions of these two field components share too many similar features, making it impossible to accurately fit the experimental signal $L_{y}$. We now continue the disentanglement of the electric and magnetic components of $L_{x}$, by investigating the horizontal cross section of the k-vector distribution for $k_{y}=0$.

\subsubsection{K-space distribution of $L_{x}$}

Figure 5.11 shows the experimental signal $L_{x}$ and the two dimensional Fourier transform of two holes with a diameter of 100 and $500 \mathrm{~nm}$, which are excited with horizontally polarized light. The Fourier transform is performed on an experimental data set, which corresponds to a physical area of 10x10 $\mu \mathrm{m}$. The dimensions of the scan area and pixel size, which 


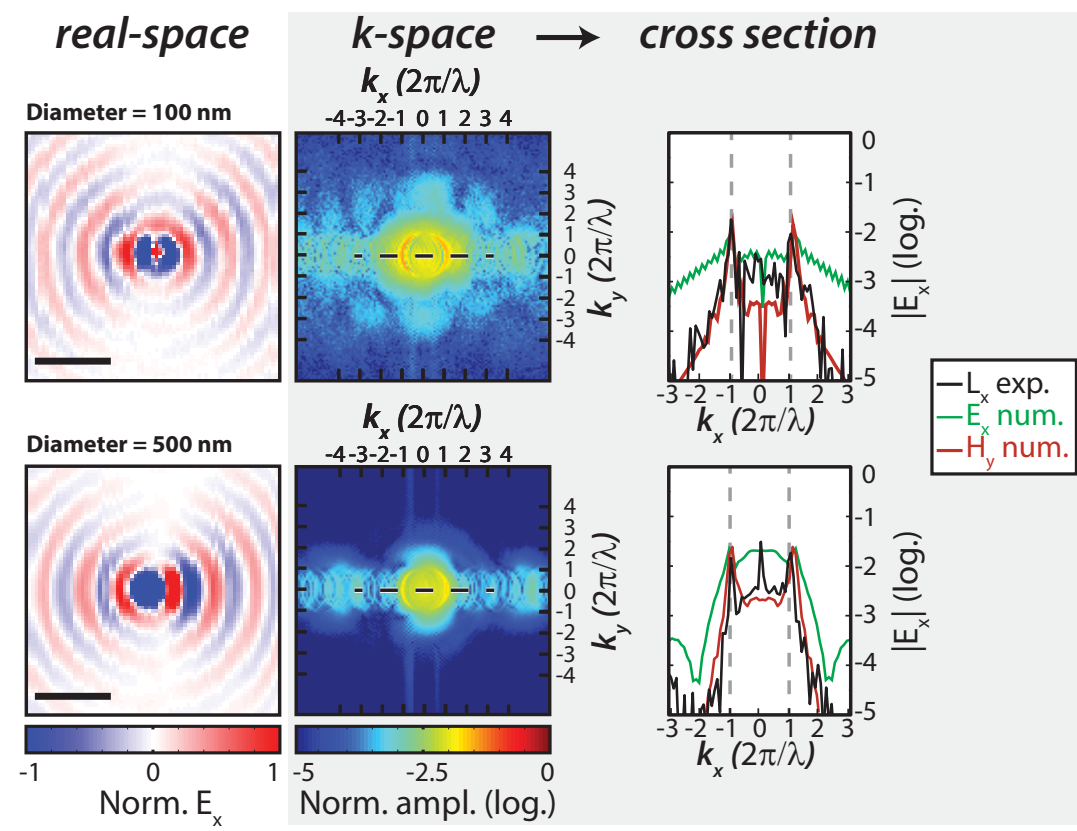

Figure 5.11: The $\operatorname{Re}\left\{\mathbf{L}_{x}\right\}$ field distribution and the two dimensional Fourier transform of two holes of different lateral dimensions. (Left) the experimental field distribution $\left(\operatorname{Re}\left\{\mathbf{L}_{x}\right\}\right)$ of two holes with a diameter of 100 and 500 $\mathrm{nm}$ are shown, from which the two dimensional Fourier transform is calculated (Center). (Right) a horizontal cross section taken from the center section of the experimental k-vector distribution, indicated with the black dashed line, is plotted together with Fourier transforms of the numerical calculations of $E_{x}$ and $H_{y}$. The vertical gray dashed lines indicate the k-vector of the SPP mode.

were used in the collection of the experimental data, are also used in the calculations of cross sections of $E_{x}$ and $H_{y}$. Because the pixels in the experiment have an estimated physical dimension of about $80 \mathrm{~nm}$, only $\left(|k|_{(x, y)}<4 k_{0}\right)$ have any physical meaning, as higher $\mathrm{k}$-vectors will have less than three pixels determining their spatial frequency, which is also known as the Nyquist criteria. The k-vector distributions for both the 100 and $500 \mathrm{~nm}$ diameter holes show similar features, which are also seen in the k-vector distributions of $E_{x}$ and $H_{y}$, of which the most predominant are the two semicircular peaks which are associated with the SPP modes. The k-vector distribution of the hole with a diameter of $100 \mathrm{~nm}$ seems to be spread out more to higher k-vectors, and faint peaks emerge around $\left(|k|_{(x, y)}=3-4 k_{0}\right)$, which are not seen in the case of the $500 \mathrm{~nm}$ diameter hole. These high k-vector components are most likely associated with strongly varying field distributions around the hole. For larger holes, this effect would be weaker as the field of the hole in the 
$\mathrm{Au}$ substrate are less localized, which also means that in k-space the spatial frequencies are reduced as well. We refer to Appendix E to view the field and k-vector distributions of a series of holes with a diameter varying from 100 to $600 \mathrm{~nm}$. A horizontal cross section through the

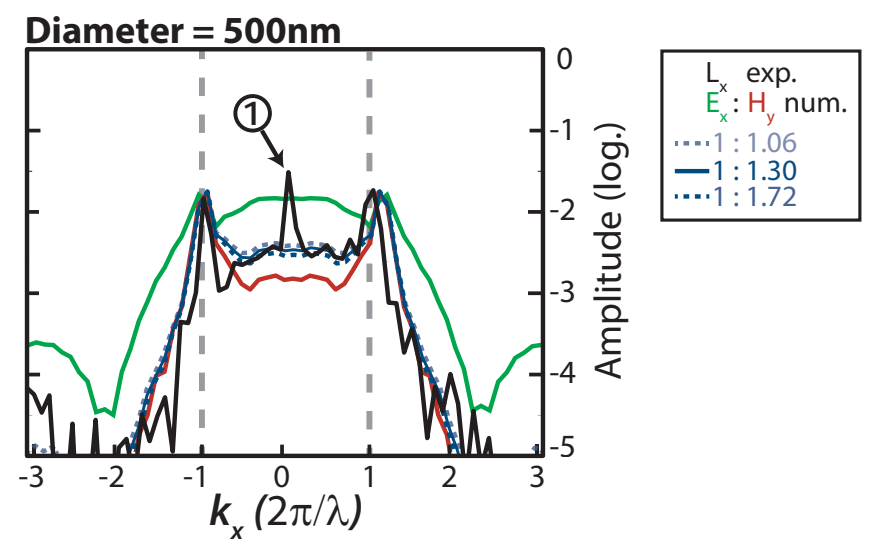

Figure 5.12: Fitting of $L_{x}$ and determining the ratio of the electric and magnetic field components. Shows the experimental k-vector cross sections of a $500 \mathrm{~nm}$ diameter hole, indicated with a black line. The experimental data is fitted (blue line) with numerical calculated k-vector distributions of $E_{x}$ and $H_{y}$, indicated with a green and red line, respectively. The upper and lower standard deviations of the fitted curve are shown with blue dashed lines. The numbered circle indicated an experimental artifact.

center section in k-space is taken, and in order to reduce noise in the experimental signal, the averaged value of five neighboring pixel columns is used. The cross sections of the experimental data are accompanied by the cross sections of the numerically calculated $E_{x}$ (green) and $H_{y}$ (red) field components. The shape of the cross sections of $E_{x}$ in the region of $\left|k_{x}\right|<1$, compared to $H_{y}$ shows that the free-space emission of the hole is dominated by $E_{x} . E_{x}$ is peaked whereas $H_{y}$ is flat. Also, the experimental cross section profiles of both holes in Figure 5.11 clearly show that they cannot be described by $E_{x}$ alone.

\subsubsection{Fitting $E_{x}$ and $H_{y}$ in k-space}

Figure 5.12 shows the fitting of the experimental cross section of a hole with $500 \mathrm{~nm}$ diameter, which is shown in Figure 5.11. The experimental data is manually fitted with a linear combination of $E_{x}$ and $H_{y}$, which is indicated with the solid blue line, together with the estimation of the upper and lower limits shown with the blue dashed lines. In the manual fitting, the Fourier transform of different ratios of $E_{x}$ and $H_{y}$ fields 
where taken and cross sections where calculated. In the fitting procedure the experimental and reconstructed Fourier transforms where normalized such that their maximum values would overlap. Only k-vectors $\left|k_{x}\right|<1.5$ are taken into account and the peak at $k_{z}=0$ was ignored. Note that in the k-vector distribution the margin of error for every $k$ vector is highly dependent on the field distribution. For example the SPP peak is constituted from the SPP fields distribution, which covers almost the whole investigated surface. From the Fourier filtering done in Figure 5.9 we know that most of the low k-vectors can be traced back to the field of plane waves emerging from the hole, meaning that the distribution of the low k-vectors is dictated by just a few data points. Also the presence of the near-field probe is not taken into account in the numerical calculations, and effectively an infinitely small probe is assumed. Which seems reasonable as the measured field distributions can be well understood in terms of the numerical $E_{x}$ and $H_{y}$ fields. The numbered circle in Figure 5.12 indicates the presence of a strong $\left(k_{x}=0\right)$ component in the experimental signal, which is not seen in the numerical calculations, which we attribute to an experimental artifact concerning the near-field probe. Because the artifact shows a k-vector equal to 0 , meaning that it shows a negligible position dependent phase, we tentatively attribute this effect to a general leakage of light into the probe, and not to a single coating defect, as seen before in Figure 5.3.

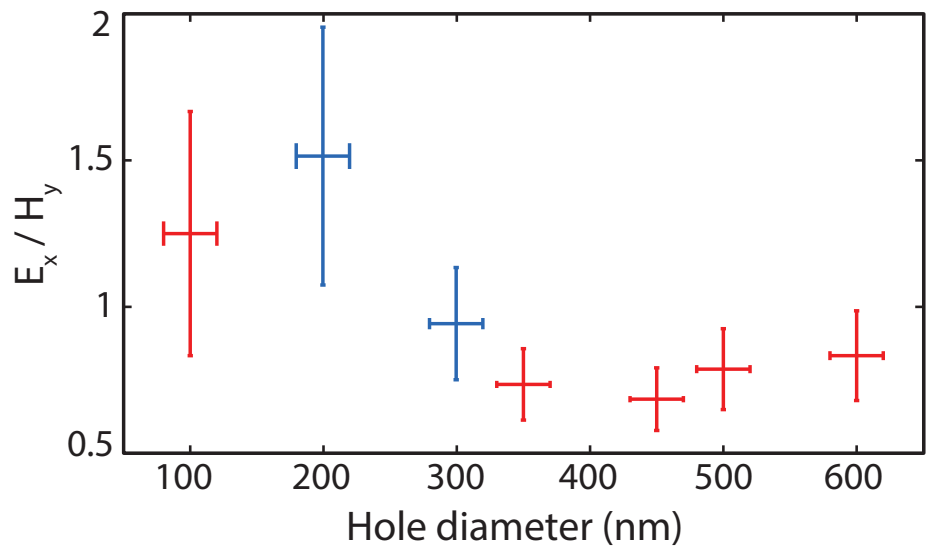

Figure 5.13: The dependence of the $E_{x} / H_{y}$ ratio on hole diameter. Experimental data on holes in a $250 \mathrm{~nm}$ thick Au substrate is fitted with numerical calculated $E_{x}$ and $H_{y}$ field distributions, from which an $E_{x} / H_{y}$ ratio is found. A range of hole diameters are studied, varying from 100 to $600 \mathrm{~nm}$. 
Finally, Figure 5.13 shows the ratio between the electric and magnetic field components for a series of holes, having a hole diameter varying from 100 to $600 \mathrm{~nm}$. The experimental k-vector distributions are individually fitted with their respective numerically calculated $E_{x}$ and $H_{y}$ field distributions. The horizontal and vertical error bars in Figure 5.13 depict the uncertainty in determining the hole diameter and the upper and lower limit of the found $E_{x} / H_{y}$ ratio, respectively. The blue data points describe the ratio of the electric and magnetic components of experimental data sets, which exhibit 5 to 10 pixels where the lock-in amplifier was saturated. We expect that, due to the saturation effects, the free-space light, which is most dominant in the $E_{x}$ field distribution, is underestimated. This effect is not seen back in Figure 5.13 where an increase of the $E_{x} / H_{y}$ ratio is observed, which is similar in magnitude to the $E_{x} / H_{y}$ ratio of a $100 \mathrm{~nm}$ diameter hole. Holes with a larger diameter $(d>350 \mathrm{~nm})$ show a constant $E_{x} / H_{y}$ ratio of approximately 0.8 .

We find an $E_{x} / H_{y}$ ratio trend which is of the same order of magnitude, showing an almost equal sensitivity to the electric and magnetic in-plane field components. These results agree well with results by Le Feber et al. in which the $E_{x} / H_{y}$ ratio dependence on the near-field aperture diameter for photonic crystal waveguides was studied [129]. In our experiments the same NSOM probe was used, and only the hole diameter in the $\mathrm{Au}$ substrate was varied. The $E_{x} / H_{y}$ trend shown for the photonic crystal waveguides, shows a slight decrease for decreasing hole diameter, while we observe the opposite trend where the $E_{x} / H_{y}$ ratio increases to approximately 1.5. We would expect that the uncertainty in determining the free-space contributions will increase for decreasing hole diameter, as less data points will determine the low k-vector values, and therefore we would expect that for decreasing hole diameter the $E_{x}$ components will be underestimated. However this would lead to a reduction of the $E_{x} / H_{y}$, rather than the increase that we observe.

\subsection{Conclusions}

In the first part of this Chapter, we have numerically investigated the transmission of light through subwavelength holes made in a $3.5 \mu \mathrm{m}$ $\mathrm{Au}$ film, where the diameter of the hole is varied around cutoff. With reducing hole diameter an increasing phase delay is found, which for a $3.5 \mu \mathrm{m}$ thick $\mathrm{Au}$ substrate can accumulate to almost $6 \pi$, albeit at a cost of amplitude. The design of phased SPP sources was discussed and first experimental results showed potential, although fabrication of the structures is still challenging. 
According to the reciprocity theorem, when applied to near-field microscopy using an aperture probe [129], it is very important to know how the near-field distribution of the used aperture probe is defined, in order to correctly interpret the NSOM data. It is for this reason, that in the second part of this Chapter, we numerically show the field distributions of subwavelength holes made in an $\mathrm{Au}$ and $\mathrm{Al}$ substrate, and demonstrate how the shape of the hole affects its field distribution.

Finally, we have experimentally investigated the near-field distributions of single holes with varying hole diameters, made in $250 \mathrm{~nm}$ thick $\mathrm{Au}$ substrates, using an NSOM. We have been able to separate the in-plane electric and magnetic components for this optical system and found a hole-diameter $E_{x} / H_{y}$ ratio dependence, which is similar in magnitude as reported in [129]. 


\section{Active control of SPP fields}

Surface plasmon polaritons are electromagnetic waves that propagate tightly bound to metal surfaces. The concentration of the electromagnetic field at the surface, as well as the short wavelength of surface plasmons enables sensitive detection methods and miniaturization of optics. In this Chapter, we present an antenna array for plasmons in which each antenna element is fully and independently controlled in both phase and polarization. Individual holes in a thick gold film act as dipolar emitters of surface plasmon polaritons (SPPs), whose phase and polarization is controlled individually using a digital spatial light modulator. We show that this approach allows accurate directional emission of surface waves and polarization tailored patterns. Our method presents a compact and flexible way to dynamically shape the propagation of plasmons and holds promise for nanophotonic applications employing propagating surface plasmons.

\subsection{Introduction}

If one sets out to control the flow of light on the smallest possible scale, surface plasmons are a natural choice, since their lateral confinement to the surface by far exceeds the confinement possible with dielectric structures. To control the flow of surface plasmons in the plane, many advances have been made over the years focusing mostly on structuring the metal surface to create waveguides, Bragg mirrors, prisms, lenses and bandgap materials for SPPs. Such strategies are promising, however, they are static in design. An approach with more flexibility has been explored by Gjonaj et al., based on spatial phase modulation on surface plasmons $[84,153,154]$. For this, a large number of holes patterned in $2 \mathrm{D}$ arrays in a metal film were used to generate plasmons, whose 
phase could be controlled to enable for instance plasmon focusing. To get the maximum out of surface plasmons, the goal is to fully control the amplitude, phase and polarization of the electric and magnetic field components and their spatial distribution with a compact and simple system. We present a minimalistic approach that achieves this. We address single holes in a metal film that act as dipole antennas for surface plasmons [155]. For each hole, we individually control phase, amplitude and polarization. We show how we can direct beams of SPPs in an analogue of a phased antenna array, and how within one $\lambda^{2}$ four spots with counter-rotating and out-of-phase circularly polarized SPPs can be created.
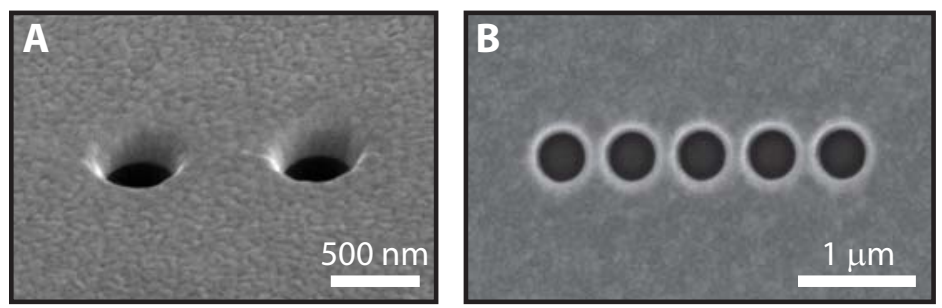

Figure 6.1: SEM micrographs of a two- and five-hole structure in a 250 thick Au film. SEM micrographs of a two $(\mathbf{A})$ and five (B) hole structure made in a $250 \mathrm{~nm}$ thick Au film, which are measured at an angle of $52^{\circ}$ and $0^{\circ}$, respectively. The gray structured surface shows the multicrystalline nature of the evaporated Au film.

Our system consists of a $250 \mathrm{~nm}$ thick film of gold, that is illuminated from below and contains a limited number of holes, see Figure 6.1. The hole size and film thickness are chosen such, that no residual light from transmission through the film is present, and that transmission of the holes is near cut-off of the lowest order mode, such that transmission through it is to occur mainly via one mode only, which is also shown in Section 5.2. Because we use individual holes, we can achieve a high level of control with a low density of holes. This also means that holehole interactions are small and can be neglected in the systems we study.

In Section 6.2, we describe two complimentary methods with which we are able to define the full optical state of light passing through the hole, meaning that amplitude, phase and polarization are separately controlled. In Section 6.3, we show how by using these methods the directionality of the generated SPPs can be altered, by making a phased antenna array. This is experimentally realized using an array of 5 holes where the phase and amplitude of the individual holes are controlled, 
and the field distributions resulting from various phase relations are shown. In Section 6.4, we investigate the field distribution above a five hole structure, illustrating how the shaped illumination also affects propagation of free-space emission, coming from the holes. In the final section, we highlight the possibilities for controlling the optical state of a hole, and we show the controlled interference of four holes leading to four spots with counter-rotating and out-of-phase circular polarized SPPs.

\subsection{Controlled generation of SPPs}

In this section, we discuss two methods which enable an increasing degree of control on the amplitude, phase and polarization state of generated SPPs. With the first method, we only use a single shaped illumination beam, and consequently only the amplitude and phase of the SPP source (hole) can be set. The second method uses two shaped illumination beams, which are $\mathrm{x}$ - and y-polarized. The amplitude and phase of both beams can be set independently, enabling the full control over the polarization state of the hole, making it possible to create elliptically polarized light, in particular linear and circularly polarized light.

\subsubsection{Amplitude and phase}

Control of the amplitude and phase state of the generated SPPs is obtained by illuminating the hole distribution with a single shaped beam of light. The method, which is used to perform both amplitude and phase shaping using a phase-only shaper, is described in Section 2.2.3. Figure 6.2 shows the SPP field distribution of two SPP sources, which are spaced $1 \mu \mathrm{m}$ apart and are illuminated by vertically polarized light. The physical structure in Figure 6.2 (B-D) and (E-G) is exactly the same, only the illumination conditions differ, where (B-D) and (E-G) are excited in- and out-of-phase, respectively. The different illumination conditions are clearly visible in $(\mathrm{C}, \mathrm{F})$ where the real part of $L_{y}$ is plotted. The two holes are individually illuminated by two dedicated SLM areas, which can be set independently. The correct amplitude and phase values for each hole are found by actually measuring the local fields with the near-field probe, which is scanned over the position of the holes. The position of the holes can also be pinpointed by looking at the error signal of the feedback loop, which controls the z-axis of the near-field probe. It is therefore possible to know, with a high degree of certainty, the exact amplitude and relative phase of each illuminated hole. When one wants to address two holes with small hole spacing (smaller than $\lambda$ ), it becomes 


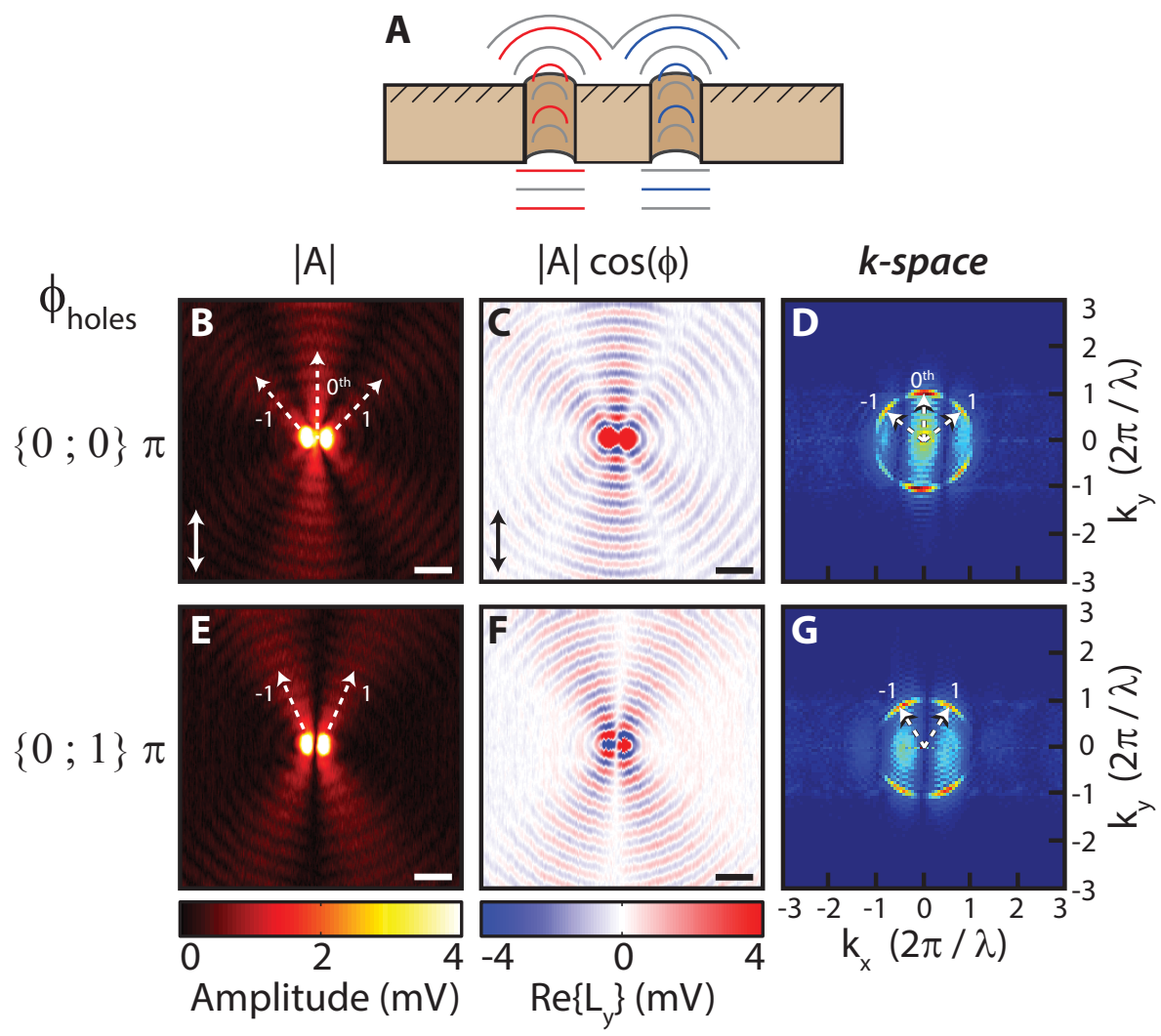

Figure 6.2: NSOM measurements of two SPP sources with control over amplitude and phase. (A) cartoon depicting the illumination of two holes which are excited with a $\pi$ phase difference. (B-D) and (E-G) show the absolute, real and Fourier transform of the experimental signal $L_{y}$, where the two holes have a phase relation of $\{0 ; 0\} \pi$ and $\{0 ; 1\} \pi$, respectively. The two holes are illuminated with vertically polarized light, indicated with the arrows in the lower left corner. The dashed white arrows indicate the propagation direction of the generated SPPs. The scale bars indicate a length of $1 \mu \mathrm{m}$.

increasingly difficult to prevent the mixing of excitation signals of those holes to occur, as the excitation beam spots start to overlap both holes. When this occurs, it is not trivial to program the correct phase pattern on the SLM, as setting the amplitude and phase for one hole influences the outcome of its neighbor. Using the collected field from the near-field probe itself as a feedback mechanism circumvents this problem entirely, enabling the holes to be positioned closer to one another. Controlling the optical state of closely spaced holes is then only limited by the resolution of the near-field probe. 
The amplitude distributions in Figure $6.2(\mathrm{~B}, \mathrm{E})$ clearly show two different field distributions when the holes are illuminated in- or out-of-phase, where the SPPs propagate outwards in six and four beams respectively. This behavior is also observed in the Fourier transform of the complex data $\left(L_{y}\right)$. The various propagation directions of the SPPs come back as six and four localized spots in k-space (panels D and G respectively).
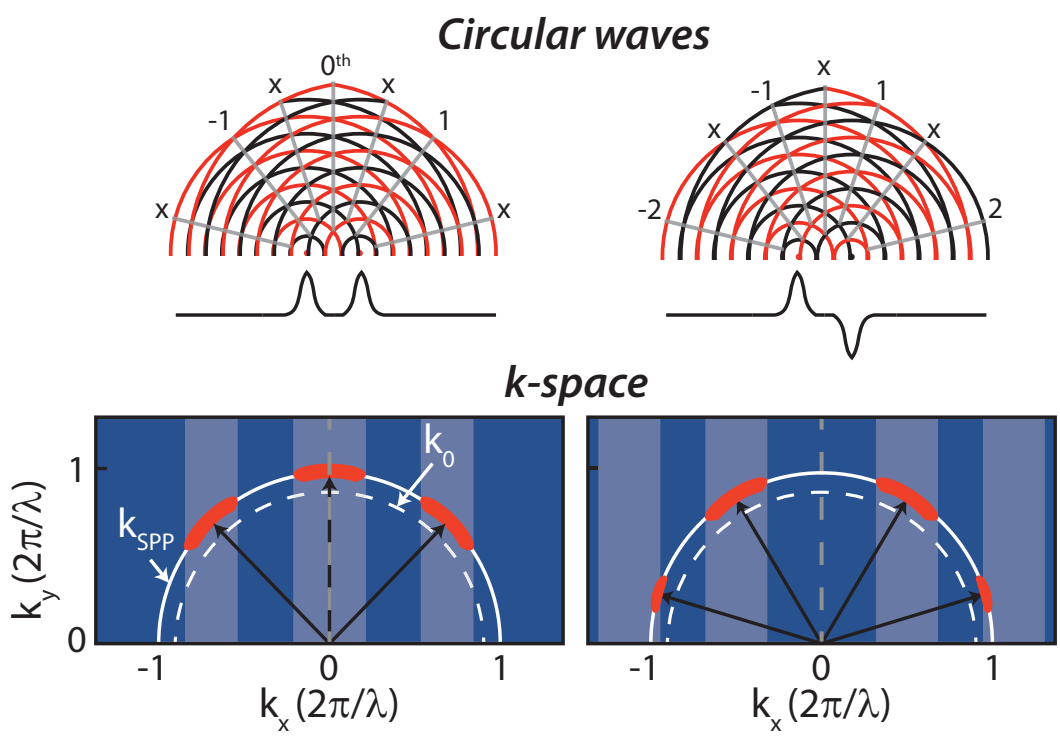

Figure 6.3: Cartoon depicting the interference of two sources of circular waves. (Above) cartoons of two circular wave sources which are in- (left) and out-of-phase (right). The resulting interference pattern shows gray lines where the waves show positive and negative interference, indicated with a number or indicated with $X$, respectively. The numbers indicate the order of the positive interference lines. (Below) cartoon depicting the k-space distribution of Figure 6.2, with the red spots indicating the direction of SPP propagation. The solid and dashed white semicircles depict the SPP wavenumber and light lines, respectively.

We use a simple model to describe the interference behavior of SPPs and the free-space emission from the linear array of holes. We consider the holes in the gold film to be dipolar point sources of surface plasmons. Then the emission pattern as collected in the y-polarized channel, a function of the azimuthal angle $\phi$, can be written as:

$$
I_{y}(\phi)=I_{0} \cos ^{4}(\theta) \frac{\sin ^{2}(N \delta)}{\sin ^{2}(\delta)}
$$

with $\delta=k_{s p p} d \sin (\theta)+\alpha, \mathrm{N}$ the number of holes, $I_{0}$ the intensity per hole, $\mathrm{d}$ the hole spacing, $k_{s p p}$ the SPP propagation constant, and $\alpha$ the 
hole-to-hole phase difference [156]. The $\cos ^{4}(\theta)$ term in this equation originates from the dipolar nature of the SPP emission, and the fact that only the $E_{x}$ and $H_{y}$ field components are picked up in the plotted detection channel.

Figure 6.3 visualizes the interference of two point sources of circular waves, which are oscillating in- and out-of-phase, respectively. The interference of two in- and out-of-phase circular wave sources shows three and four lines of positive interference, respectively. This simple representation already shows similarities to the field distribution of Figure 6.2. Note that in both cases, the sources are positioned approximately one wavelength apart. Because SPPs don't show a perfect spherical intensity, but rather a dipolar emission shape, explains why only two of the four positive interference lines are observed in the actual experiments in Figure 6.2 (E).

The lower part of Figure 6.3 shows a cartoon of the k-space distribution of Figure 6.2, where the red spots indicate the direction of SPP propagation. The solid and dashed white semicircles depict the SPP wavenumber $\left(k_{S P P}\right)$ and light-line $\left(k_{0}\right)$, respectively. In the introduction of this thesis it has been explained that the SPPs, due to their nature as surface waves, have a wavenumber which is larger than the light-line. As a consequence, SPPs show an evanescent tail in the outof-plane direction and no free-space light can directly couple to SPPs. When representing the in-plane k-space distribution, we therefore see that $k_{S P P}$ is larger than $k_{0}$. The k-vectors which lie below the light-line $\left(\mathrm{k}_{x}^{2}+k_{y}^{2}<\mathrm{k}_{0}^{2}\right)$, are not associated with SPPs but rather with free-space emission. The k-vectors which lie above $k_{S P P}$ are associated with localized near-fields, which are predominantly associated with the fields near the holes. Finally in Figure $6.2(\mathrm{D}, \mathrm{G})$ we observe lines in the free-space emission, which we have represented in Figure 6.3 as vertical blue-gray columns. These lines can be explained due to the fact that we take a Fourier transform of two point sources, which results in a sinusoidal pattern in $\mathrm{k}$-space with a periodicity of $2 \pi / d$, with $\mathrm{d}$ being the hole spacing.

\subsubsection{Controlling the polarization state}

In order to obtain polarization control, two excitation beams with orthogonal polarization are used to illuminate a single hole, which is schematically depicted in Figure 6.4 (A). Because the amplitude $\left(A_{1,2}\right)$ and phase $\left(\phi_{1,2}\right)$ distributions of each polarization beam are shaped independent of one another, any given polarization state can be formed. When the 

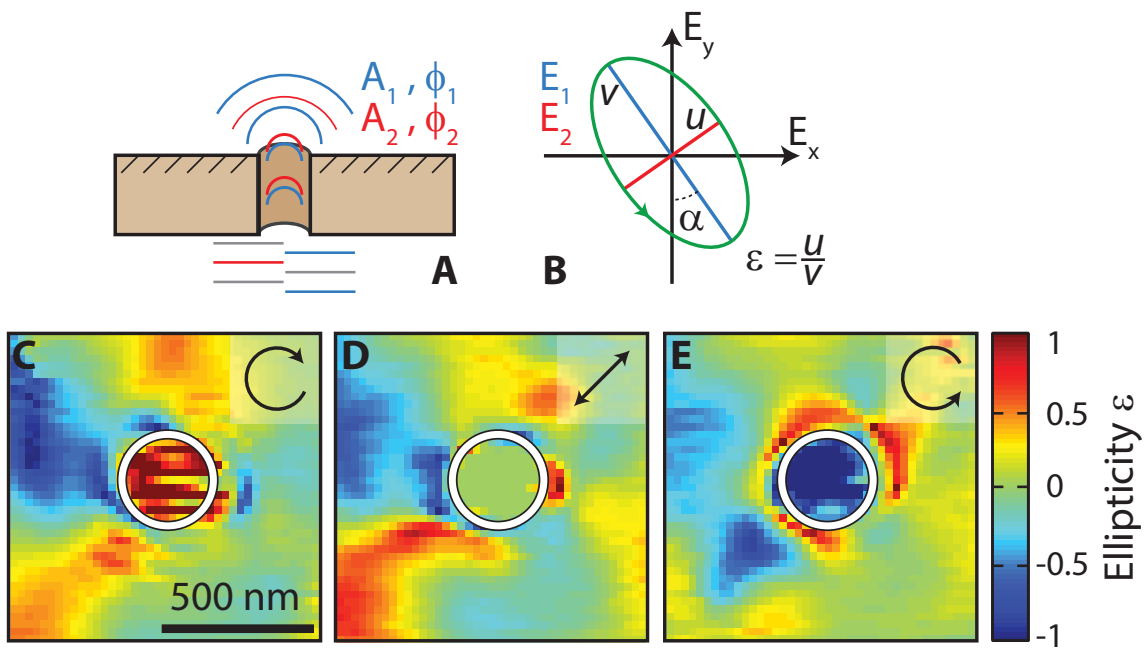

Figure 6.4: Controlling the polarization state of a single hole. (A) cartoon depicting the excitation of a single hole using two beams with orthogonal polarization. (B) the polarization ellipse, which is formed by the two field components $\left(E_{1}=v\right)$ and $\left(E_{2}=u\right)$. (C-E) polarization resolved NSOM measurements showing the ellipticity $(\epsilon)$ of a single hole when illuminated with left-hand circular, linear, and right-hand circular polarized light. The position of the hole is indicated with a white circle.

polarization beams have the same phase $\left(\phi_{1}=\phi_{2}\right)$, but different magnitude $\left(A_{1} \neq A_{2}\right)$, will result in a linearly polarized beam where the polarization angle is determined by the ratio of the amplitudes $\left(A_{1} / A_{2}\right)$. Varying the phase difference of the polarization beams from 0 to $\pi / 2$ results in light which linearly, elliptical and circularly polarized. A measure for the ratio $(\epsilon)$ and orientation $(\alpha)$ of the polarization ellipse is given by $[157-159]$ :

$$
\begin{aligned}
\epsilon(r) & =u / v=\tan \left\{\sin ^{-1}[\sin (2 \psi(r)) \sin (\delta(r))] / 2\right\} \\
\alpha(r) & =\left\{\tan ^{-1}[\tan (2 \psi(r)) \cos (\delta(r))]\right\} / 2
\end{aligned}
$$

where $v$ and $u$ represent the mayor and minor semi-axis of the polarization ellipse (Figure $6.4(\mathrm{~B})$ ). The angles $\psi=\tan ^{-1}\left[\left|E_{y}\right| /\left|E_{x}\right|\right]$ and $\delta=\delta_{y}-\delta_{x}$ characterize the amplitude ratio and phase difference between the two field components. The ellipticity $\epsilon$ ranges from -1 to +1 , corresponding to right- and left-hand circularly polarized light, respectively. Burresi et al. used this description to visualize polarization singularities in the near-field of photonic crystal waveguides, which where measured with a similar polarization sensitive NSOM [158]. Figure 6.4 (C-E) shows polarization resolved NSOM measurements of the ellipti- 
city $(\epsilon)$ of a single hole, indicated with a white circle, when illuminated with left-hand circular, linear and right-hand circular polarized light. The geometry is kept constant and only the illumination conditions are varied. The fields above the hole clearly vary from left- to right-hand circularly polarized light, indicated with the red and blue areas within the encircled area. Although the ellipticity value above the holes follow a clear trend, the values surrounding the hole are more ambiguous, and we don't reveal any clear trend. We speculate that the asymmetric ellipticity distribution is caused by the use of a probe, which shows an angle dependent polarizablity. Although an absolute value for the ellipticity is not obtained, Figure 6.4 does show that with the shaping method, full control over the measured polarization state is obtained. The NSOM measurements of the ellipticity of the near-field distributions shown, later in this Chapter, strongly depend on the quality of the near-field probes. The way we define the ellipticity of the measured signals implicitly assumes a near-field probe, which is equally polarizable from every in-plane direction. Biased ellipticity measurements are obtained when deformed near-field probes, where the aperture for instance does not form a ring but rather an ellipse, are used. The only way to correct for this unwanted effect is by having a priori knowledge of the optical system to identify and discard biased probes. This is common practice when working with an NSOM. The ellipticity distributions shown in the rest of this thesis have been measured with various probes giving repeatable results.

\subsection{Controlling the directionality of SPPs}

In this section, we focus on two methods which provide control on directionality of propagation and the directivity of SPPs. First we study the effects of design and how control on the propagation direction can be obtained by changing the hole spacing. In the second part of this section we investigate a plasmonic phased antenna array consisting of 5 holes.

\subsubsection{Control by design}

First we investigate how the propagation of SPPs depend on a geometrical factor, namely the spacing between two holes. Our experiment is very similar to the two slit experiments of Thomas Young in 1801, but in our case we use two holes and investigate the near-field distribution of SPPs instead of the far-field. As shown previously, using the phase sensitive information of the NSOM, we can calculate the k-vector 

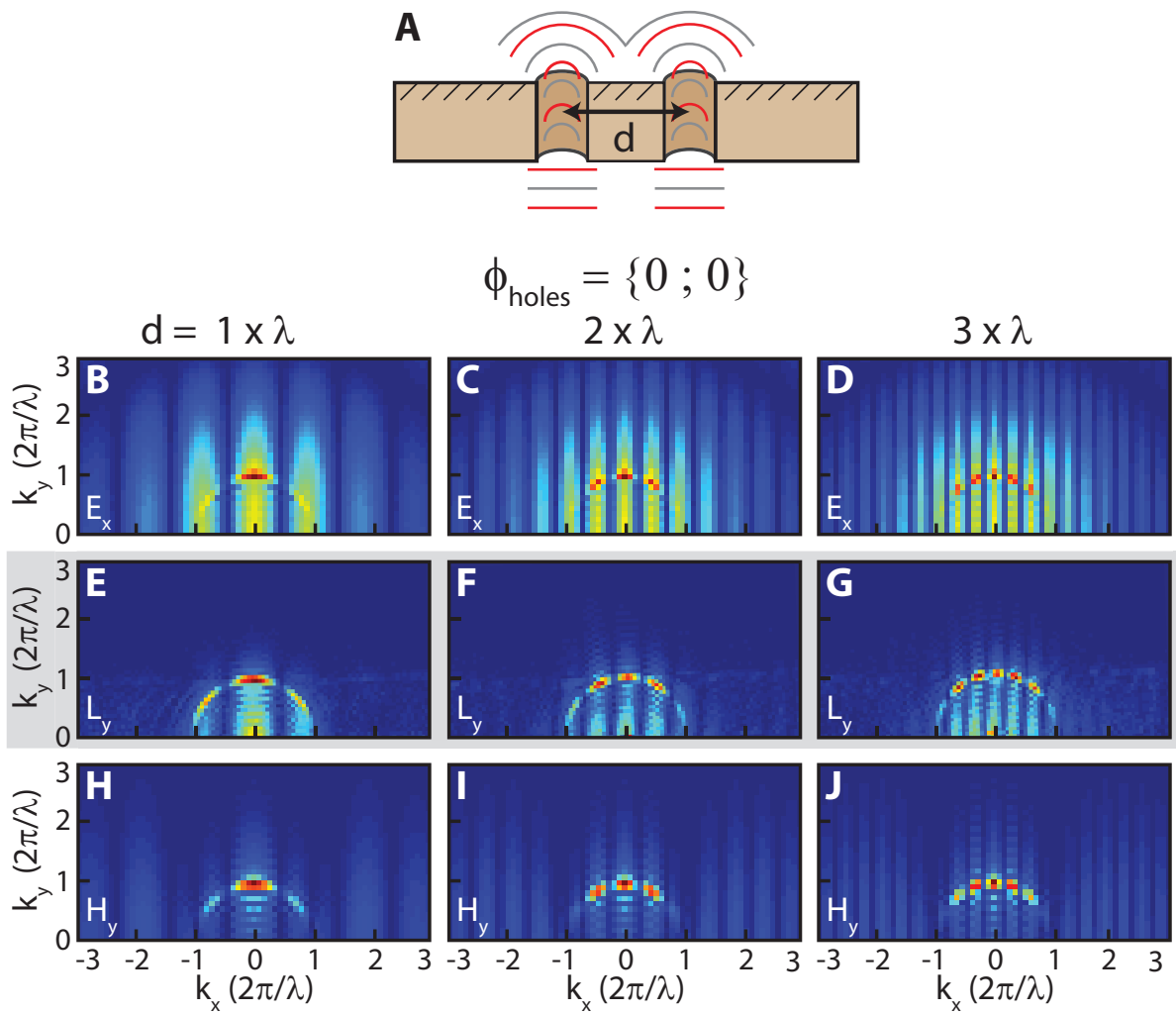

Figure 6.5: The effect of hole spacing on k-vector distributions. (A) cartoon depicting the illumination of two holes which have a hole spacing $d$. (B-D), $(\mathbf{H}-\mathbf{J})$ and $(\mathbf{E}-\mathbf{G})$ show numerical $\left(E_{x}\right.$ and $\left.H_{y}\right)$ and experimental $\left(L_{y}\right)$ k-space distributions, respectively, of the interference patterns of two holes having a hole spacing $(d=1,2,3 \lambda, \lambda=950 \mathrm{~nm})$.

distribution above a sample surface. The k-vector distributions we obtain in the NSOM measurements contain information of both the SPPs' field distribution and the free-space emission. It is therefore possible to compare the free-space diffraction pattern, and the k-space distribution of the propagating SPPs in a single NSOM measurement. Figure 6.5 (B-D), (H-J) and (E-G) show numerical $\left(E_{x}\right.$ and $\left.H_{y}\right)$ and experimental $\left(L_{y}\right) \mathrm{k}$-space distributions, respectively, of the interference patterns of two holes having a hole spacing $(d=1,2,3 \lambda, \lambda=950 \mathrm{~nm})$. Only the $\mathrm{k}$-vector distribution of $K_{y}>0$ is shown, as the distribution is mirror symmetric. The experimental data (E-G) shows that with increasing hole spacing, the periodicity of the direction of propagation of the SPPs increases. With a hole spacing of $(d=1,2,3 \lambda)$ we observe 3,5 and 7 $\mathrm{SPP}$ peaks in the k-space distributions, which also follows from Equa- 
tion 6.1. The angular redistribution due to the increase in hole distance, is visualized in Figure 6.6, where the amplitude distributions on the line $k_{S S P}^{2}=k_{x}^{2}+k_{y}^{2}$ are plotted as a function of angle. This representation clearly shows that with increasing hole spacing, not only the periodicity of the SPP peaks increases, but also their directivity, where the peaks become more narrow. This becomes evident with a hole spacing of $d=3 \lambda$, where the peaks have a larger aspect ratio.

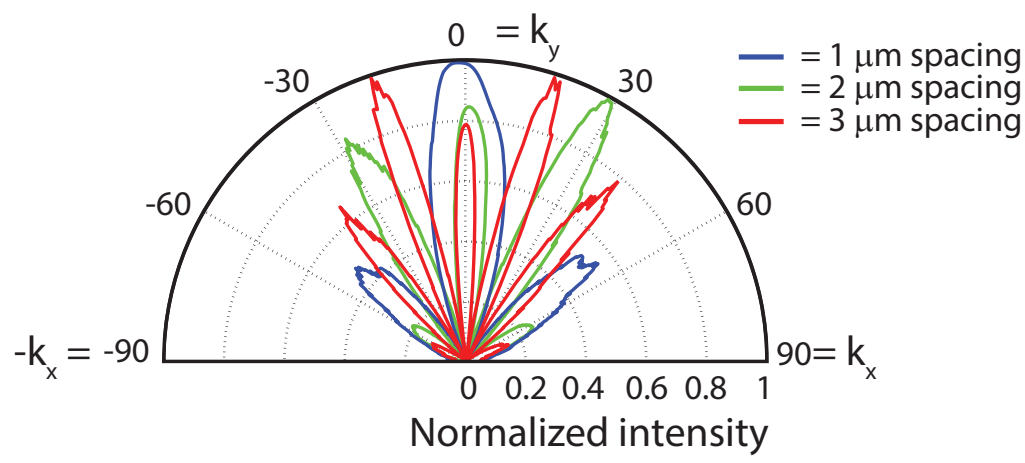

Figure 6.6: Angular distribution of SPP propagation directions of a two hole system. Polar plot showing the angular distribution of SPP propagation directions for a two hole system having 1,2 $3 \lambda$ hole spacing and $\phi_{1}=\phi_{2}$. The polar plots are obtained by calculating the Fourier transform of NSOM amplitude distributions, after which we plot $\left|k_{S P P}\right|^{2}$.

Also for lower k-vector values, which lie under the light-line, a periodicity with a similar period to those of the SPPs is observed. In Section 6.2.1 we have discussed these periodicities and attributed them to the interference of free-space components, which can be understood when considering that effectively we are looking at the interference of two delta-like point-sources. The position of the maxima are governed by $2 \pi / d$, where $\mathrm{d}$ is the hole spacing. This relation is also clearly found in Figure 6.5, where for example (F) shows its maxima at approximately $k_{x}=-1 ;-0.5 ; 0 ; 0.5 ; 1$.

Along with the experimental data, we also plot numerical calculations of the electric $\left(E_{x}\right)$ and magnetic $\left(H_{y}\right)$ in-plane field components. Comparing the numerical EM field distributions with $L_{y}$, it immediately becomes apparent that neither $E_{x}$ nor $H_{y}$ alone can describe $L_{y}$, and like we have seen in Section 5.4, a combination of both $E_{x}$ and $H_{y}$ is needed. In the k-vector distribution of $H_{y}$, a higher contrast between $k_{S P P}$ and $k_{0}$ is observed, compared to the k-vector distribution 
of $E_{x}$. This ratio is well understood in terms of the impedance of the SPP fields $\left(Z_{S P P}=E_{x_{S P P}} / H_{y_{S P P}}\right)$ compared to the free-space emission $\left(Z_{0}=E_{x_{0}} / H_{y_{0}}\right)$, where in Chapter 1 we show that $Z_{S P P} \approx 0.16 Z_{0}$. This means that for the measured SPP fields $H_{y}$ has a stronger contribution compared to the experimental signals, which contain free-space emission.

\subsubsection{Active control}

We now discuss the second method to manipulate the propagation of SPPs, where the illumination conditions are actively varied in order to obtain the desired SPP distribution. A row of five holes with a hole spacing of $d=\lambda=950 \mathrm{~nm}$ is used to make a phased array of SPP sources. The holes are individually illuminated and their amplitude and phase are actively controlled. In Figure 6.7 we apply three different
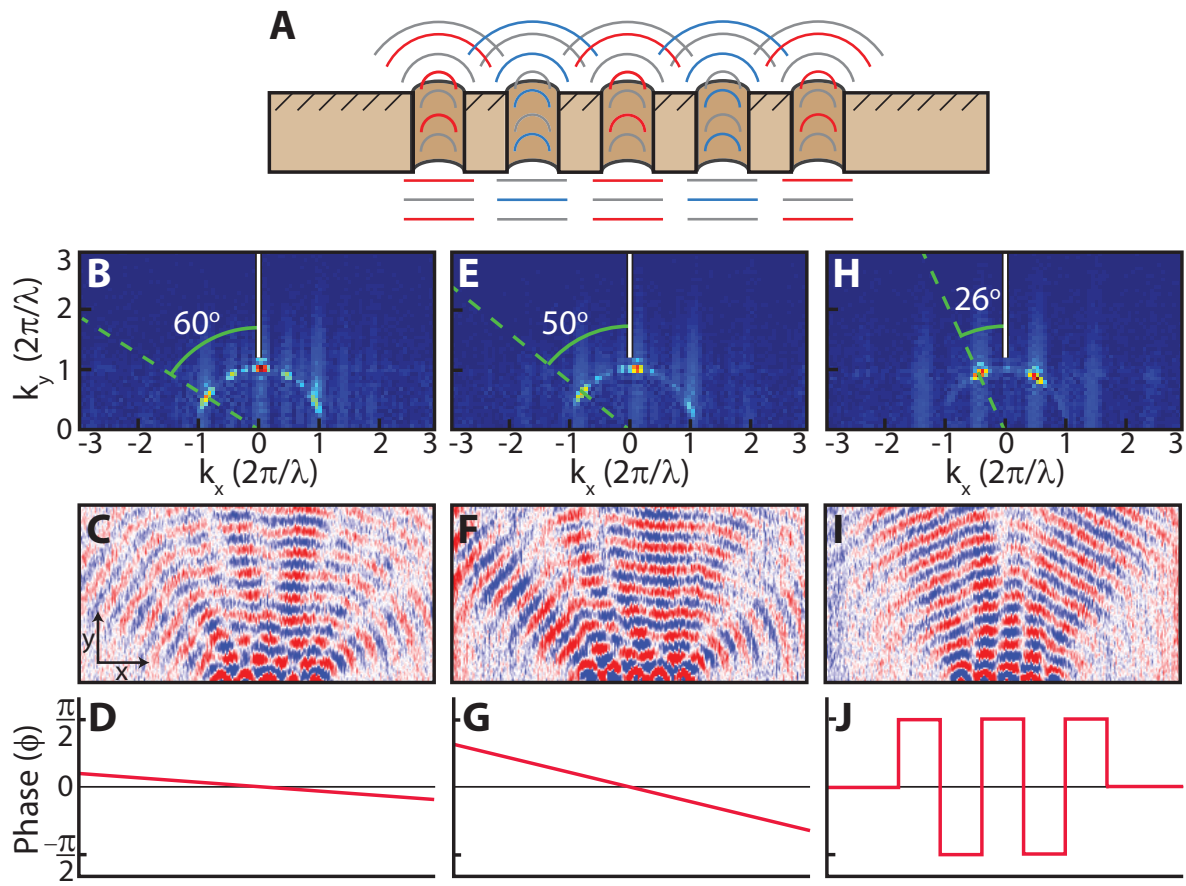

Position

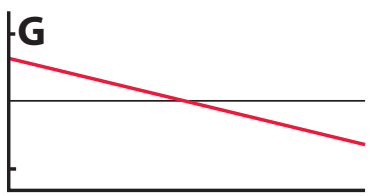

Position

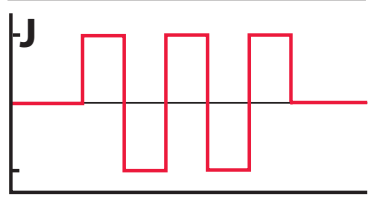

Position

Figure 6.7: Phased array of SPP sources. (A) cartoon depicting the illumination of five holes, which are excited with an alternating phase of $-\pi / 2$ and $\pi / 2$. In (B-D), (E-G) and (H-J) three phase slopes are used which, respectively vary from, almost flat, slightly angled, to the most extreme phase slope possible, where the phase of adjacent holes vary from $-\pi / 2$ to $\pi / 2$. Moving from above to below, the k-space, real components of the field distribution, and an illustration of the phase patterns of the holes are shown. 
phase slopes, which show an increasing slope angle. By using an NSOM we are able to relate the phase gradients at the holes with the resulting field distribution. In Figure 6.7 (B-D), (E-G) and (H-J), we show three phase slopes, respectively varying from almost flat, slightly angled, to the most extreme phase slope possible, where the phase of adjacent holes varies between $-\pi / 2$ to $\pi / 2$ (see the illustrations Figure $6.7(\mathrm{D}, \mathrm{G}, \mathrm{J})$ ). The k-vector distributions of $(\mathrm{B}, \mathrm{E})$ show that increasing the angle of the phase slope (see $D, G$ ) results in a tilt in the propagation direction of the SPPs by $10^{\circ}$. The most extreme tilt of the SPP propagation direction is obtained in (H and I), which both show the two SPP beams propagating at an angle of approximately $-26^{\circ}$ and $28^{\circ}$, where the model predicts the two maxima to be on $\pm 27^{\circ}$.

\subsubsection{Manipulating the directivity of SPPs}

Besides the directionality of SPPs, the directivity is also a property of interest for many applications, like the miniaturization of plasmon microscopes $[154,160]$. Following conventional definitions in antenna theory [161], the directivity of waves propagating in a 2D plane can be defined as:

$$
D_{\max }=2 \pi \frac{U_{\max }}{P_{\text {rad }}}
$$

where $U_{\max }$ describes the maximum radiation intensity, and $P_{\text {rad }}$ describes the total radiated power. From this it follows that the directivity of a circular wave is equal to 1 , and the directivity of a dipole $\left(\sin ^{2}(\phi)\right)$ will be equal to: $\frac{2 \pi}{\int_{0}^{2 \pi} \sin ^{2}(\phi) d \phi}=2$. The angular distribution of the generated SPPs of a single hole, are well described by a dipolar distribution $[10,128,162]$. In this section we will investigate the angular distribution of SPPs generated from arrays of holes, where the number of holes, the hole spacing and the phase distributions of the holes, are varied, and we will show that the directivity of such hole structures can become much larger than the directivity of single hole.

There are effectively two methods to accurately derive the directivity for the optical system we are studying. First of all, one could investigate the NSOM amplitude distribution on a wide circle around the hole structures, effectively in the 'far-field' of the SPPs. The second method involves taking the Fourier transform of the complex data set and investigating the angular distribution of the SPPs in k-space. Both methods in principle are equally good, although for the first method one has to be sufficiently far away from the structure to make sure that the physical dimensions of the structure do not affect the outcome. Also, method 
one suffers from the fact that the propagation of the SPPs is strongly damped, which limits the area one can investigate to approximately 100x100 $\mu \mathrm{m}$. We therefore have chosen to investigate our NSOM data in k-space, which yielded more accurate results for the directionality and directivity of the hole arrays, as this does not suffer from the same problems as the first method. The resolution obtained in k-space is inversely proportional to the dimensions of the area investigated, meaning that most of $\mathrm{k}$-space distributions we show in this thesis have a resolution of $1 / 10 \lambda$.

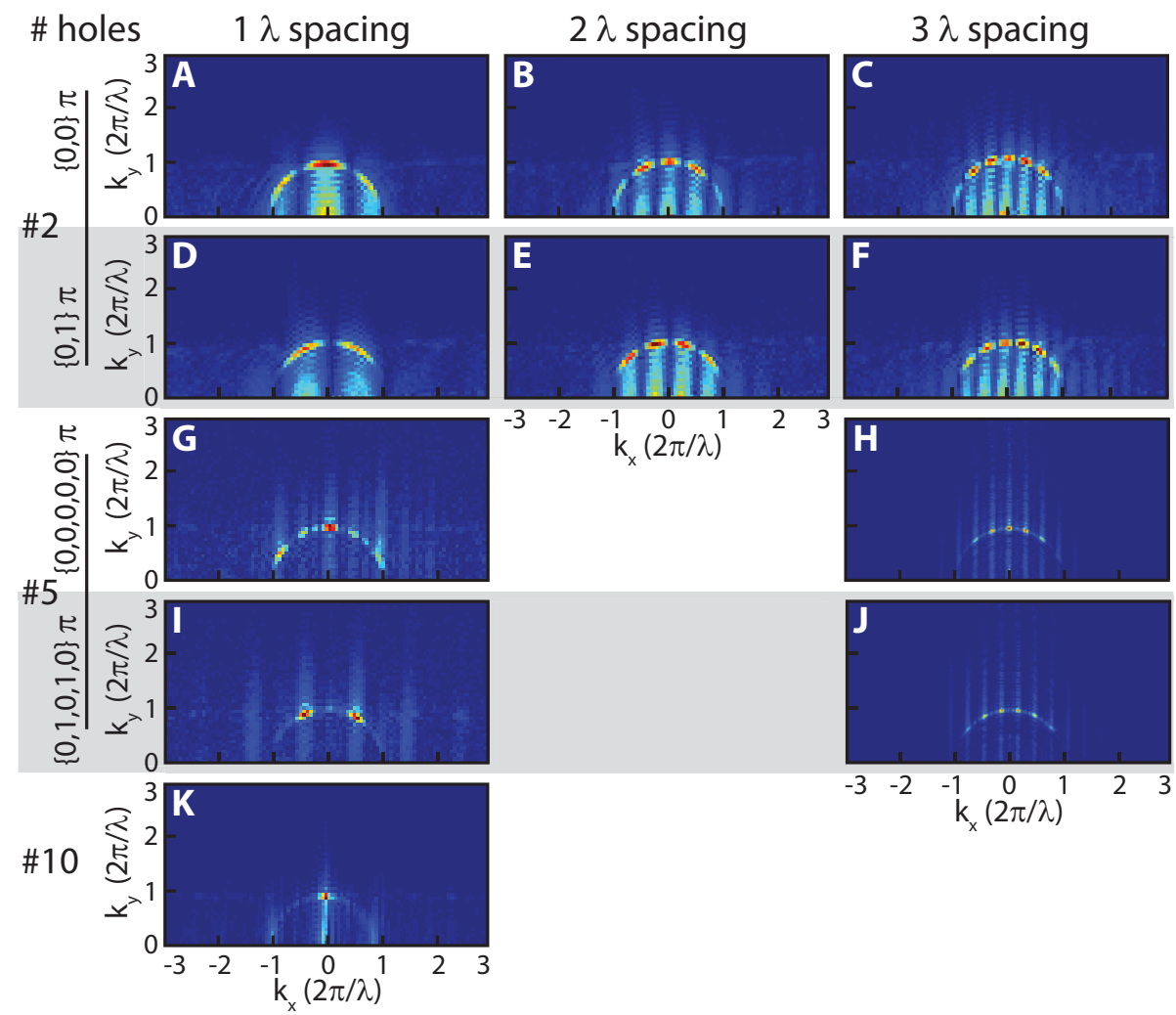

Figure 6.8: Wavevector distribution illustrating the directivity of SPPs generated from arrays of $\mathbf{2 , 5}$ and $\mathbf{1 0}$ holes. (A-F), (G-J) and (K) k-vector distributions, calculated from measured data of arrays of 2, 5 and 10 holes, respectively. The hole arrays show an even phase distribution and a phase distribution which alternates between 0 and $\pi$ for adjacent holes, indicated with white and gray rows, respectively. (A-C) show k-vector distribution which have also been shown in Figure 6.5 
Figure 6.8 shows the k-vector distributions of various configurations of holes, which are measured using the NSOM. The resolution of the kvector distributions of $(\mathrm{H}, \mathrm{J})$ are clearly higher compared to the other distributions, which can be explained by the fact that for these measurements an area of 30x30, instead of 10x10 $\mu \mathrm{m}$ was investigated. In order to be sure that the $\mathrm{k}$-space distribution is not undersampled we apply zero-padding of eight times the number of samples taken.

First, for increasing hole spacing new peaks start to emerge, showing a decreased width, which is nicely demonstrated in Figure 6.8 (A-C). Increasing the hole spacing effectively decreases the directivity, as more of the radiated power is dispersed into more peaks, however the peaks become more narrow, which leads to an increase in directivity. The net result on directivity of these counteracting effects is shown in Table 6.1. Secondly, applying an alternating phase distribution on the hole array, which varies between 0 an $\pi$, an even (instead of an odd) number of peaks are observed. From Table 6.1 this is shown to lead to a slightly reduced directivity. Finally, a relation between an increasing number of holes which are collectively driving the SPPs, and a decreasing width of the $k_{S P P}$ peaks is observed, which is nicely demonstrated in $(\mathrm{A}, \mathrm{G}, \mathrm{K})$. To demonstrate how the directivity scales with the number of holes which are simultaneously driven, we show the $k_{S P P}$ of a series of hole arrays consisting out of 1,2,5 and 10 holes and having a hole spacing of $\lambda$, see Figure $6.9(\mathrm{~A})$. The black dashed line in (A) indicates the emission pattern of a dipole. The polar plots of the NSOM data are obtained by extracting the $\mathrm{I}\left(k_{x}, k y\right)$ on the line parametrized by $k_{S P P}^{2}=k_{x}^{2}+k y^{2}$, which are shown in the $\mathrm{k}$-space distributions of Figure 6.8 , noting that $k_{S P P}$ lies on a semicircle in k-space, which is illustrated in Figure 6.3. The polar plots clearly show that with an increasing number of holes, the directivity strongly increases, where a directivity of 17.5 is found in the case of the 10 hole structure, which is almost six times larger than the directivity of a single hole (see Table 6.1). The increase in directivity values is induced in two fold as the peaks at $0^{0}$ become more narrow and the side peaks, which are still quite dominantly present in the two-hole structure, decrease in size with increasing number of holes in the structure.

The polar plots in Figure 6.9 (B-E) are plotted on a logarithmic scale, in order to visualize to what extent the model can describe the smallest features of the SPP propagation patterns of the different hole structures. The NSOM SPP propagation patterns of a single hole (B), and of groups of 2, 5 and 10 holes (C-E) are compared to the model, without the use of 


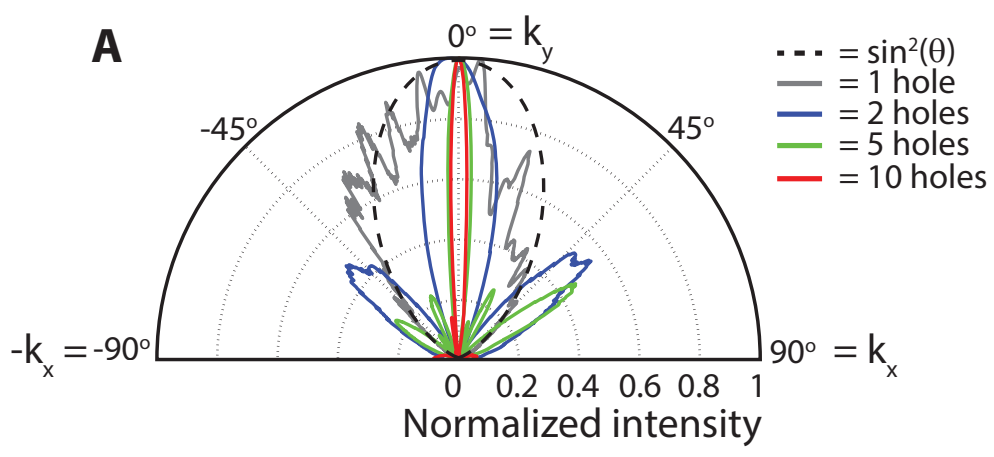

Logarithmic scale
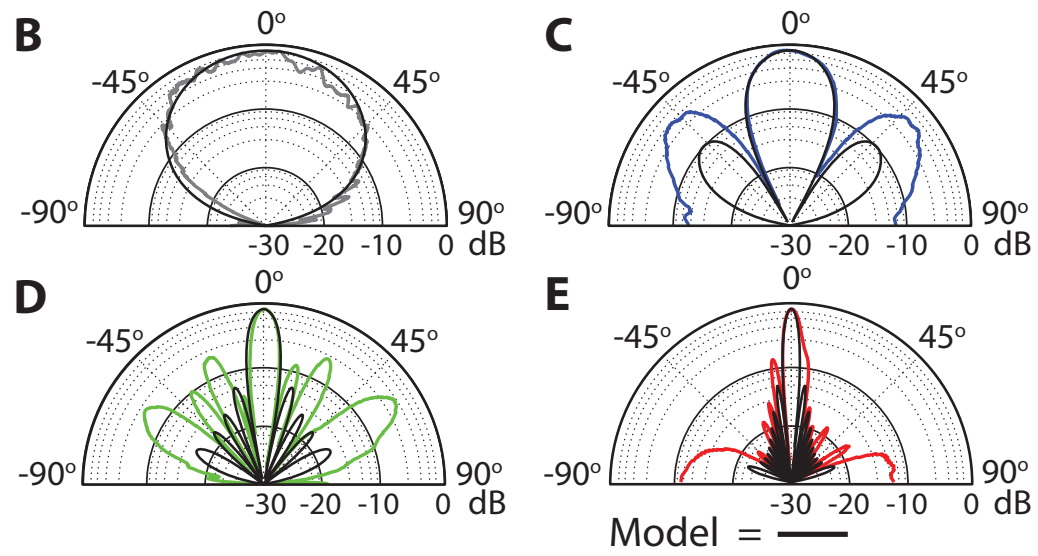

Figure 6.9: Directivity of SPPs generated by hole arrays consisting out of $1,2,5$ and 10 holes. (A-E) polar plot illustrating the angle of propagation of SPPs generated by a single hole and arrays of 2, 5 and 10 holes, which are compared to a $\sin ^{2}(\theta)$ line, resembling the emission of a dipole. The experimental data is plotted on a linear (A) and logarithmic (B-E) scale, in order to highlight the directivity of the data and the accuracy of the model, respectively. The polar plots are obtained from the absolute squared Fourier transform of $L_{y}$.

any free parameters, and are plotted with a black line. The general shape of the angular pattern of different hole structures are well reproduced by the model, where peaks at $0^{\circ}$ are very well reproduced by the model. Also the angle of the smaller side peaks seen in the experimental data are well reproduced, although their magnitudes, especially for $|\theta|>70$, are found to be systematically lower for the model. We argue that the discrepancy between the model and the experimental $k_{S P P}$ values can be explained by the fact that we implicitly assume that the intensity distribution, which forms the $k_{S P P}$ semicircle in k-space, is solely formed 


\begin{tabular}{l|cccc}
\hline \# holes (phase) & - & $\mathrm{d}=1 \lambda$ & $\mathrm{d}=2 \lambda$ & $\mathrm{d}=3 \lambda$ \\
\hline $\mathbf{1}$ & $3.0 / 2.7$ & - & - & - \\
$\mathbf{2}\{0,0\}$ & - & $3.3 / 5.7$ & $3.6 / 5.4$ & $3.8 / 5.3$ \\
$\mathbf{2}\{0, \pi\}$ & - & $3.2 / 3.3$ & $3.4 / 4.7$ & $3.2 / 5.0$ \\
$\mathbf{5}\{0,0,0,0,0\}$ & - & $7.1 / 14.9$ & - & $14.1 / 13.4$ \\
$\mathbf{5}\{0, \pi, 0, \pi, 0\}$ & - & $7.1 / 7.2$ & - & $11.0 / 12.6$ \\
$\mathbf{1 0}$ & - & $17.5 / 30.6$ & - & - \\
\hline
\end{tabular}

Table 6.1: Directivity of a single hole and of arrays of holes containing 2, 5 and 10 holes. The directivity of SPP distributions which are obtained from NSOM measurements on a single hole and arrays of holes containing 2, 5 and 10 holes. The experimental data is compared to directivity values, obtained from a model which describes the interference of multiple dipolar sources (see Section 6.2.1), indicated in red. The directivity of different hole spacing (d) and phase distributions $(0,0)$ and $(0, \pi)$ are shown.

by SPPs. This does not necessarily have to be true, as any field distribution having the correct spatial periodicity can end up on $k_{S P P}$. If the positioning of the holes themselves show a periodicity which is equal to $\lambda$, this means that the free-space emission coming from the holes already provides a periodicity in $\mathrm{k}$-space, which also has a contribution to $k_{S S P}$; see also Section 6.2.1. In the current configuration the holes are illuminated with vertically polarized light, meaning that the free-space emission is strongly present in the xz-plane. Therefore, only the larger angles of the experimental k-vector distribution in Figure 6.9 should be affected most. Increasing the scanning area of the NSOM measurements would reduce this effect, as the ratio of the free-space to SPP contributions would reduce. Due to the dipolar nature of the SPP generation of single holes, we have shown that this model is capable of describing, in great detail, the angular pattern of linear hole arrays. This means that this model can be used for the design of passive and active hole arrays of arbitrary length, hole spacing and phase distributions. 


\subsection{Shaping light in the z-axis}

We now investigate the field distribution above the surface, where a mixture of both evanescent and free-space emission fields are present. We have seen how by changing the phase distribution of five holes one can manipulate the direction of propagation of the SPPs, and we will now show how the field distribution above the sample surface is also affected. We show the field distribution of five holes, which are illuminated in such a way that they show an alternating phase distribution, varying between $-\pi / 2$ and $\pi / 2$.

Figure $6.10(\mathrm{~A}, \mathrm{~B})$ shows NSOM data of the absolute value and real part of the field distribution on the sample surface, $\mathrm{z} \approx 10 \mathrm{~nm}$. The measurement of the volume above the sample is obtained by making a line-by-line scan of the surface, where for each line a height scan is made, whereby the topography of the surface is also taken into account in the z-positioning of the probe. Because the height measurements are done per line trace, we minimize the drift in the z-axis. A typical measurement of a volume of 20x20x2 $\mu \mathrm{m}$ (xyz), takes between two and four hours, meaning that the measured phase has to be stable within that time frame. Any disturbances or drift in the phase will be predominantly visible in the xy-plane. Figure $6.10(\mathrm{C}-\mathrm{F})$ shows height measurements of the cross sections in $(\mathrm{A}, \mathrm{B})$, indicated with blue and green dashed lines. In these measurements, the height varies between in contact $( \pm 10 \mathrm{~nm})$, and $1.2 \mu \mathrm{m}$ above the surface. The alternating phase pattern of the hole array is well recognizable in $(\mathrm{D})$.

Figure $6.10(\mathrm{C}, \mathrm{E})$ shows how the free-space emission leaves the hole in the metal film, where one can observe a broad light cone, which is indicated with blue dashed lines in (E). In Section 1.4.1 we have shown that the evanescent decay length $\hat{z}=1 /\left|k_{z}\right| \approx 100 \mathrm{~nm}$, meaning that at a height of roughly $300 \mathrm{~nm}$ the magnitude of the SPP fields have reduced to $5 \%$ of the field strength present on the gold-air interface. Because the evanescent SPP fields above the surface are purely imaginary, the phase of these fields are constant as a function of height. It is interesting to note that outside the bright cone, indicated in (E) with the blue dashed lines, also fields showing spherical phase fronts are observed. As the phase of the evanescent fields are constant, we know that these fields are also free-space emissions which leave the hole under a very steep angle, shearing the sample surface. From Chapter 1 we know that the phase velocity of the SPPs is lower compared to the free-space emission $\left(\lambda_{S P P}=0.988 \lambda_{0}\right)$, meaning that after 40 oscillations at the surface the 
free-space emission and the SPPs will be out-of-phase with one another. This could explain the small kink in the phase front near the sample surface, which is observed in $(\mathrm{F})$, where near the surface $(\approx 10 \mathrm{~nm})$ the SPP fields are dominant and 200-300 $\mathrm{nm}$ above the surface the free-space emission is dominant. The SPP fields and the free-space emission could potential interfere within $300 \mathrm{~nm}$ above the surface, however in the case of hole-antennas both components are in-phase.
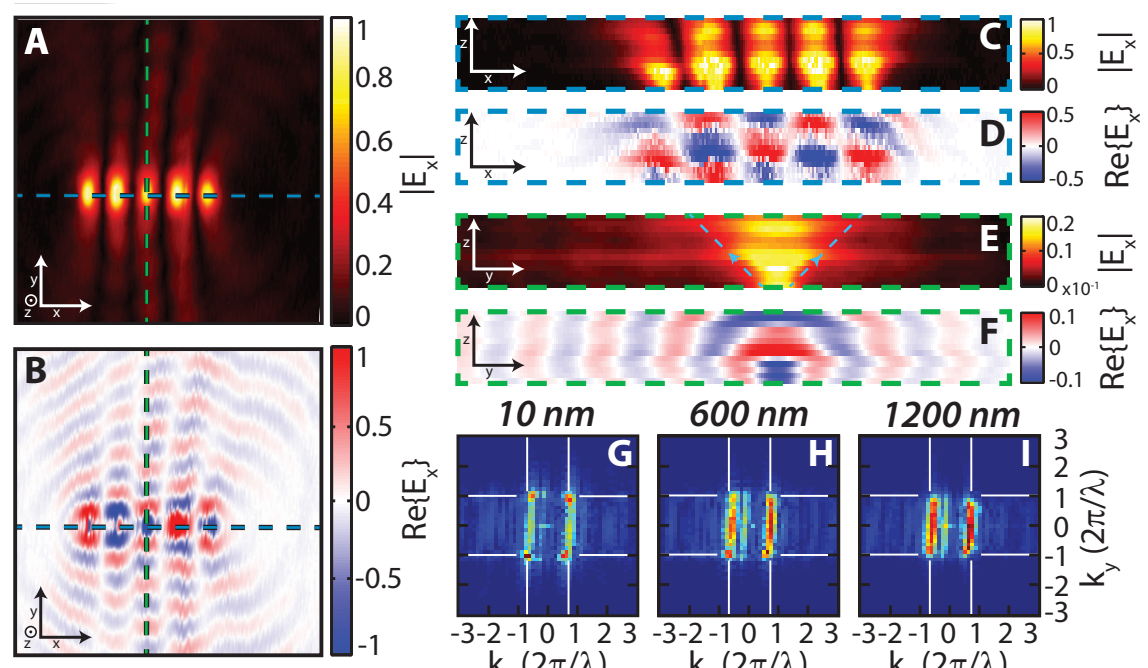

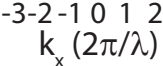
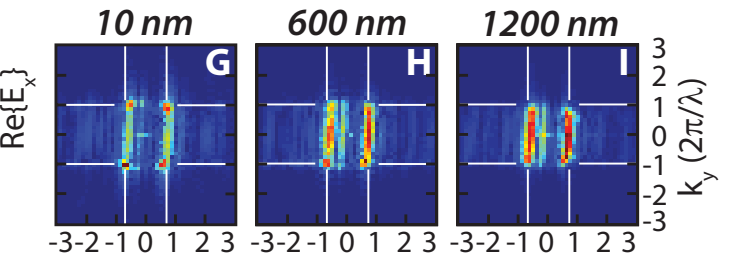

Figure 6.10: Height NSOM measurements on hole array. This figure shows the field distribution of an array of five holes which are measured at heights varying from 10 to $1200 \mathrm{~nm}$ above the sample surface. The holes are illuminated such that holes have alternating phases of $-\pi / 2$ and $\pi / 2$. (A,B) Show the absolute and real components of $L_{y}$, measured when the NF probe is in contact $( \pm 10 \mathrm{~nm}$ above surface). (C-F) shows height measurements of the cross sections in $(A, B)$, indicated with blue and green dashed lines. (G-I) are k-vector distributions at 10 , 600 and $1200 \mathrm{~nm}$, which are normalized to their own respective maximum value, and the white lines indicate the $\mathrm{x}-\mathrm{y}$ components of $k_{S P P}$.

In Figure 6.10 (G-J) one can observe how as a function of height, the $\mathrm{k}$-space distribution changes from effectively four peaks in $(\mathrm{G})$, which are associated with the SPPs, to two thick lines in (H,I). Note that the $\mathrm{k}$-vector distributions are normalized to their own respective maximum values, which is done to enhance the contrast, but it does not mean that the free-space intensity increases with increasing height. The SPP lobes in $(\mathrm{G})$ have higher k-vectors than the free-space emission lines in (I), where the white lines indicate the $\mathrm{x}-\mathrm{y}$ components of $k_{S P P}$. The $\mathrm{k}$-vector distributions now experimentally show that the difference be- 
tween $k_{S P P}$ and $k_{0}$ is very small. Also the evanescent character of the SPP is nicely illustrated in the three k-vector distributions, where in $(\mathrm{G})$ the k-vector distribution is dominated by the SPP lobes, in $(\mathrm{H})$ the free-space emission and SPPs fields are roughly equal, and in (I) the free-space emission is dominant. Figure 6.10 (I) illustrates how by shaping the phases of the holes, the free-space emission is also shaped, whereby the two lines in $\mathrm{k}$-space are shifted by $\pi / d$, compared to the situation where the holes are illuminated with a flat phase front.

In Figure $6.10(\mathrm{C})$, above the holes we observe an amplitude modulation as a function of height. We further investigate this effect in Figure 6.11 (A), where we plot the average value of an area which encloses the five holes for the different positions in height. Two maxima are observed which are spaced roughly half a wavelength apart. This effect could for instance be explained if a standing wave is formed between the NSOM probe and the hole itself. Another explanation could be that this is not a measurement artifact but an interference effect of multiple holes. Figure $6.11(\mathrm{~B}, \mathrm{C})$ shows a close-up of the amplitude distributions of the
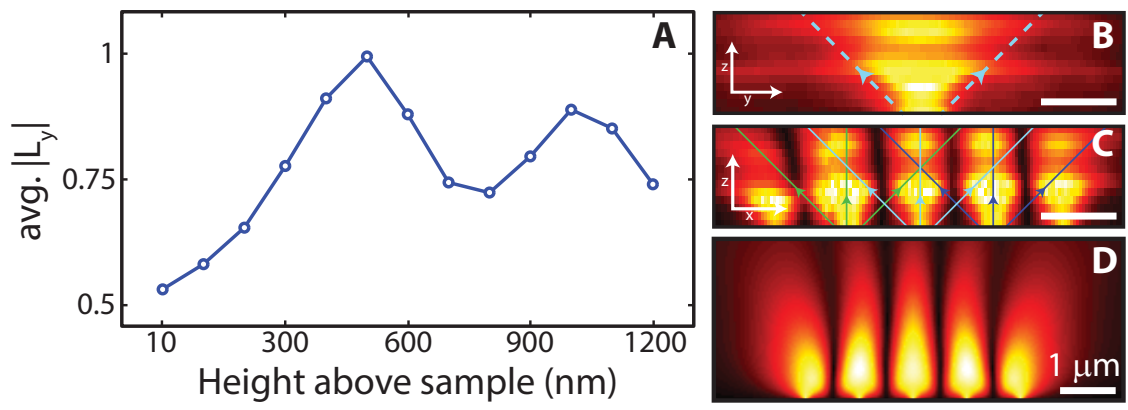

Figure 6.11: Height-dependent amplitude modulation. (A) the average value of an area which encloses the five holes, measured at different positions above the surface. $(\mathbf{B}, \mathbf{C})$ a close-up of the amplitude distributions of the cross section in Figure 6.10 (C,E), where the dashed lines indicate the cone shape of the free-space emission. (D) the amplitude distribution calculated with a simple model, which represents the holes as point dipoles which are oscillating out-of-phase. The scale bars indicate a length of $1 \mu \mathrm{m}$.

cross section in $6.10(\mathrm{C}, \mathrm{E})$, where the dashed lines again indicate the cone shape of the free-space emission. When the cone emission lines of three holes are plotted over the amplitude distribution in (C), we observe that the free-space emission lines of the center three holes overlap, which means that the observed modulation could still be an interference effect. The local maxima which arise due to the interference of multiple 
sources, is also referred to as the Talbot effect $[163,164]$, which has been observed with NSOM measurements of an array of holes illuminated with a plane wave [165]. To determine whether the amplitude modulation is real or not, we can vary the phase of the holes, as this should change the positions of positive and negative interference; however, we haven't tried this.

In Figure 6.11 (D) we show a calculation of five dipole sources, which show an alternating phase jump of $-\pi / 2$ and $\pi / 2$, and a hole spacing of $\lambda$. A simple model is used where the field distributions of the holes are represented as dipole like sources, emitting spherical wave patterns, and no SPP components are considered. The amplitude distribution at a height varying from 0 of $3 \mu \mathrm{m}$ is calculated, revealing that for a hole spacing of $\lambda$, the amplitude distribution does not show any modulations in the z-direction. This suggests that the observed amplitude modulation in Figure 6.11 is indeed a probe-sample artifact. We only observe an amplitude modulation right above the holes, where the intensity is maximum. As the intensity of spherical waves drop off with the radius cubed, we expect this effect only to occur close to the holes. For the near-field measurements when the probe is in contact $(z \approx 10 \mathrm{~nm})$ we do not observe any reflection artifact, indicating that the SPPs are less affected by the presence of the probe, compared to the free-space emission. This probably has to do with the fact that for the SPPs the probe is orthogonal to the direction of propagation, while for the free-space emission the probe is orientated parallel to the direction of propagation.

\subsection{Circular polarized SPP fields}

In the previous sections, we have shown how by shaping the illumination of hole arrays, one can tune the direction of propagation and the directivity of generated SPPs. In this section, we will go one step further and describe a method with which it is possible shape the optical polarization state at any given location, on the sample surface and above, which we then verify with an NSOM. Four holes are used as local sources to generate SPPs and free-space emission, and they are placed in a crossshape, as shown in Figure 6.12. First we will use a simple model, based on the interference of spherical waves, to calculate the field distributions for four different hole-phase combinations. After this, we experimentally verify one of the calculated hole-phase combinations, and show that indeed with this method patches in the xy-plane of left- and right-hand circular polarized light are be obtained. Finally we experimentally explore the volume above the sample surface. 


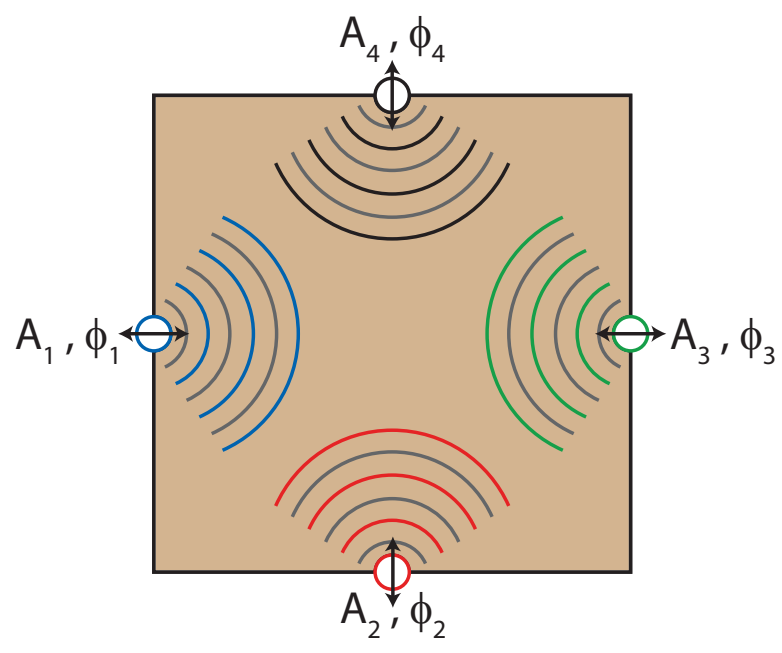

Figure 6.12: Cartoon depicting the four-hole system. A cartoon depicting the hole and illumination configuration for controlling the polarization state, at a point in the hole enclosed surface. Holes $(1,3)$ and $(2,4)$ are illuminated with horizontal and vertical linearly polarized light, respectively, which is indicated with the black arrows above the holes.

As the aim is to realize an EM field with circular polarization, it is good to realize that an SPP is a partially longitudinal TM wave. In no way can a combination of SPPs propagating in the same direction lead to circular polarization in the plane. Therefore it is necessary to have SPPs interfering under different propagation directions. We use the following geometry: four holes, $(1,3)$ and $(2,4)$, are illuminated with horizontal and vertical linearly polarized light, respectively, where the amplitude and phase of each hole are set individually. Horizontal and vertical SPP standing wave patterns contain both the in-plane electric and magnetic field components $E_{x, y}$ and $H_{y, x}$, respectively (see Section 5.4). However the SPP field distribution of $E_{x, y}$ and $H_{y, x}$ are almost identical, and therefore in the rest of the discussion we do not make the distinction. For the sake of clarity, in the rest of the discussion we refer to $L_{x, y}$ when describing experimental signals in the $x$ and $y$ polarization axis, which contains both the electric and the magnetic in-plane field components. The standing wave pattern of the holes $(1,3)$ and $(2,4)$ are horizontally and vertically polarized, meaning that they will predominantly be measured in $L_{x}$ and $L_{y}$, respectively. The phase difference of the hole pairs determines where in space positive and negative interference occurs. This then provides a method to vary the position where, for instance, patches 
of elliptical or circularly polarized light occur. In the situation where the holes are closely spaced $(\leq \lambda)$, the amplitude ratio and phase difference of the group of holes, $(1,3)$ and $(2,4)$, determine whether predominantly linearly, elliptical or circularly polarized light occurs on the surface, enclosed by the holes. Because the holes are point sources of SPPs, the $\mathrm{SPP}$ field is not simply the interference of two standing wave patterns. Therefore, though the center region is quite well described by a standing wave pattern, the outer regions are not. Figure 6.13 (B-E) shows calculated ellipticity plots of four combinations of phases, with which the four holes are illuminated. In these calculations the SPPs field distributions, which are generated from holes, are simplified as spherical waves, which effectively means that the field components which are orthogonal to the polarization axis are not taken into account. So for example, when the hole is illuminated with linearly polarized light in the x-direction, only $E_{x} / H_{y}$ is taken into account. This simplification is chosen because it is able to describe the most important features in the center of the structure.

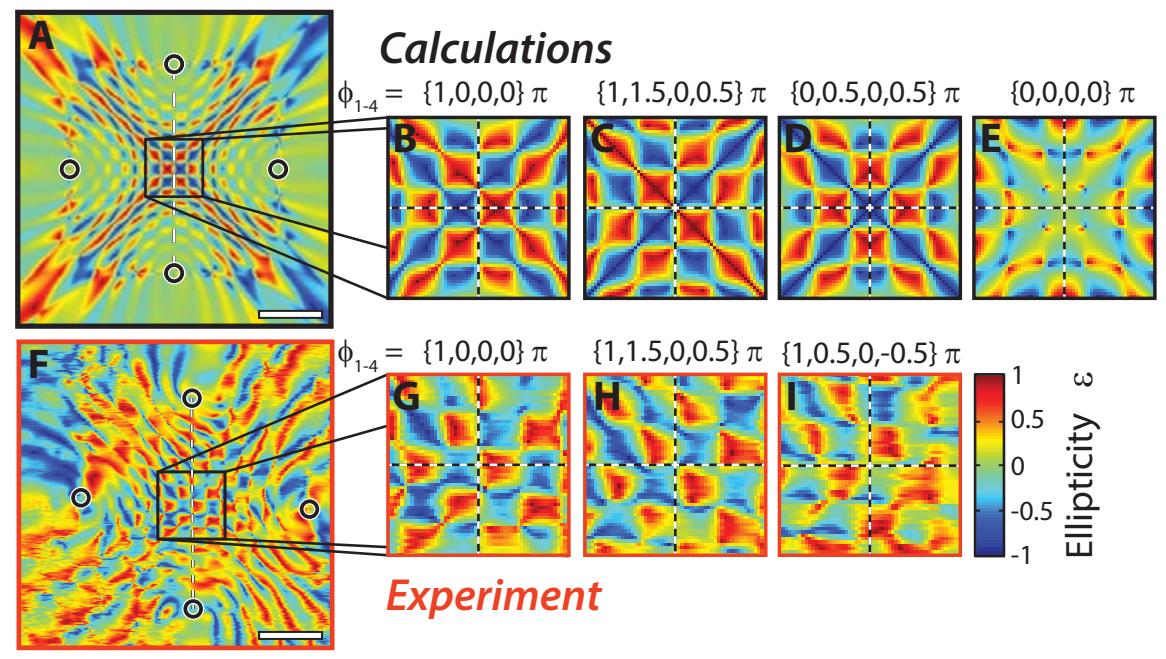

Figure 6.13: Ellipticity patterns of four holes with various hole-phase combinations. (A-E) calculated ellipticity plots of four hole-phase combinations, assuming that the SPP field distribution can be simplified to the interferences of spherical waves. (F-I) NSOM ellipticity distributions of three different holephase combinations. The white dashed lines are a guide to the eye, indicating the symmetry of the ellipticity distributions. The scale bars indicate a length of $2 \mu \mathrm{m}$. 
Figure 6.13 (A-C) shows calculated ellipticity distributions of a four hole system, where the phase distributions of the holes are chosen such that a checker board pattern of left- and right-hand circularly polarized light emerges. The patches are evenly spaced around the center, showing only vertical anti-symmetry, which is indicated with a white dashed line in (A). This four-hole system with similar amplitude, phase and polarization relations is experimentally measured using an NSOM (F-G), where we find a striking resemblance of the symmetry of the ellipticity distributions. The zoom-in of the experimental results is most accurately described by calculations, as the area in between the holes can be accurately described by circular waves. The NSOM measurement compared to the calculations shows more ellipticity features, leaving the holes at $45^{\circ}$ angles, which is due to the fact that in the model only the field components parallel to the polarization axis are taken into account. These weaker, orthogonal field components actually have a radiation pattern, where SPPs are generated with a propagation direction which is diagonal compared to the excitation polarization, see Figure 5.8. If now an additional phase shift of 1.5 and $0.5 \pi$ is applied for the holes 2 and 4 , we obtain the distribution shown in $(\mathrm{C}$ and $\mathrm{H})$, where the ellipticity distribution becomes point anti-symmetric. The phase distribution of the four independent holes in figure (I) are chosen such that the same the same ellipticity distribution as $(\mathrm{H})$ is constructed, however the patches with left- and right-hand circular polarization are interchanged. These three experimental results show that a checker board pattern of left- and right-hand circularly polarized light can obtained, by using the standing wave patterns of four holes. Additionally they show how the ellipticity distribution can be moved in space. With that in mind we also show the calculated ellipticity distributions of (D), where the illumination conditions are chosen such that the vertically polarized holes have a phase shift of $\pi / 2$, resulting in a point symmetric checker board motief. In Figure 6.13 (E) all the holes are illuminated in-phase, meaning that all the waves arrive in the center of the image, all with the same phase delay, and consequently in the center the ellipticity shows a value of zero, meaning that at that position the light is linearly polarized.

Figure 6.14 shows a side by side comparison of the ellipticity distributions of the SPPs propagating on the surface (A), and of free-space emission at $1600 \mathrm{~nm}$ height (B). Both distributions are taken in a single experiment and the illumination conditions are the same for both images. Interestingly, also above the sample surface the alternating circular polarization patches continue with the same symmetric distribution as 
on the surface. Also, the field distribution at a height of $1600 \mathrm{~nm}$ shows more similarities to the model in Figure 6.13 (A,B), than our NSOM data when in contact. This has to do with the fact that at a height of $1600 \mathrm{~nm}$ we only observe free-space emission and no field contributions of SPPs. This shows that the free-space emission coming from the holes can very well be described by the propagation of simple spherical waves.
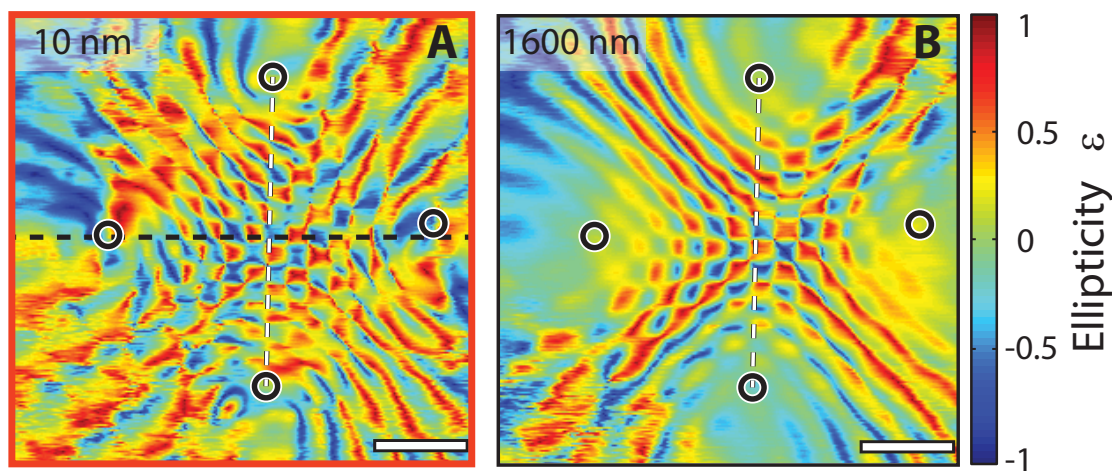

Figure 6.14: NSOM measurements of localized circularly polarized fields. NSOM measurement showing the elliptisity distribution of SPPs and free-space emission, measured on (A) and $1.6 \mu \mathrm{m}(\mathbf{B})$ above the sample surface, respectively. The four holes are illuminated with a $\phi_{1,2,3,4}=\{1,0,0,0\} \pi$ phase distribution, similar to Figure $6.13(\mathrm{~F})$. The black and white circles indicates the position of the holes. The scale bar indicated a length of $2 \mu \mathrm{m}$. The white dashed line is a guide to the eye indicating the anti-symmetry line of the ellipticity distribution. The black dashed line indicates the position where the cross section is taken, shown in Figure 6.15.

Figure 6.15 (A, B and $\mathrm{C}$ ) shows a vertical cross section, which in Figure 6.14 is indicated with a black dashed line, of the absolute, real and ellipticity distribution, which are taken at 10 to $1200 \mathrm{~nm}$ height above the surface. In the vertical cross section of $\mathrm{A}$ and $\mathrm{B}$ we observe a standing wave pattern in between the position of two holes, which can be recognized in (A) by the two bright cones of light. The standing wave shows an alternating phase, but more interestingly, Figure 6.15 (C) shows that the standing wave pattern has an alternating circular polarization handedness, forming closely spaced columns of circularly polarized light. Figure (D) shows the polarization angle at a point in time for four pixels, which are located in the circularly polarized patches in the center of the image, which are then measured at various heights. The polarization angles show that the circular polarized patches, which are left- $(1,3)$ and right-handed $(2,4)$, and are $180^{\circ}$ out of phase. 


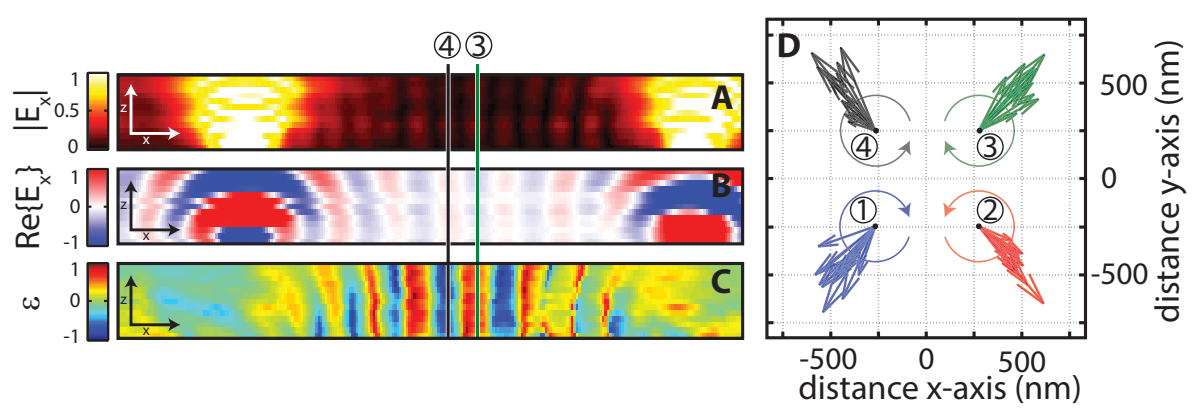

Figure 6.15: $(A, B, C)$ the absolute, real and ellipticity distribution of height measurements of the horizontal cross section shown in Figure 6.14, and indicated with a black dashed line. (D) the polarization angles measured at different heights above the sample, which indicate the polarization at a point in time.

\subsection{Conclusions}

In the first part of the Chapter, we have demonstrated the controlled generation of SPPs, which was accomplished by means of designing and actively manipulating the illumination conditions. The amplitude, phase and polarization state of every hole-antenna in 2,5 and 10 element hole arrays are addressed individually, making it possible to manipulate the emission of the generated SPPs. The directionality and directivity of the various hole arrays have been investigated and reconstructed with a simple model. We have shown that by applying a linear phase-slope with various slope angles, the directionality of the generated SPPs can be altered significantly. Besides investigating the field distributions of the SPPs on the Au-air interface, we have also shown NSOM measurements of the field distribution of free-space emissions above a hole array, measured at various heights. These experiments illustrated how the free-space emission can also be shaped using this technique, making it possible to actively control the directionality of free-space emission coming from arrays of holes. Finally, we have demonstrated an optical system consisting of four holes, for which the optical states are individually controlled. Two standing SPP waves are generated, having orthogonal polarization, leading to a checker board pattern of left- and right-hand circularly polarized light. We have experimentally demonstrated that by varying the relative phase relation of the two standing waves, it is possible manipulate the position of the checker board pattern, showing our capability to control the ellipticity state at given locations. The capability of controlling the polarization state, and in particular the handedness of circularly polarized light, could be very interesting for the 
analysis of low concentrations of chiral molecules which are sensitive to certain degrees of circular dichroism, or for controlling the spin-state of quantum systems, such as quantum dots. 
$\Gamma_{\text {Appendix }}$

\section{Fabrication techniques}

For the experimental verification of the fundamental concepts described in this thesis, it is of vital importance to have access to state-of-the-art nanofabrication facilities. Because the processes described in this thesis take place on a length scale which is much smaller than the wavelength of light used in our experiments (600 - $1000 \mathrm{~nm})$, nanofabrication techniques capable of making structures with a spatial resolution of \pm 10 $\mathrm{nm}$ are needed. In this appendix the basic concepts of the relevant fabrication techniques, as well as key details needed to obtain structures with $10 \mathrm{~nm}$ resolution, are described. First the electron-beam (e-beam) lithography process is described, which is used to fabricate the bar-ring structures in section 4.2. Next, the synthesis of single crystalline gold flakes, and its use as a substrate for focused ion beam (FIB) fabrication is described, which is used to fabricate the modulated antennas in chapter 3 .

\section{A.1 Electron-beam lithography}

E-beam lithography is a nanofabrication technique capable of making structures with a sub $10 \mathrm{~nm}$ resolution, which is done by scanning an electron beam over a surface coated with a resist layer. From left to right, Figure A.1 summarizes the consecutive process steps, which are needed in fabricating a structure using e-beam lithography. A resist layer with the desired thickness is applied (step 1), which is often done using a spin coater in a room with controlled humidity and temperature. For the bar-ring structures in Section 4.2, a positive resist (ZEP520A) was chosen, for which a minimum feature size of $10 \mathrm{~nm}$ has been demonstrated [166]. By exposing a positive resist to a beam of electrons, the local bonds between the individual resist molecules are broken (step 2), 


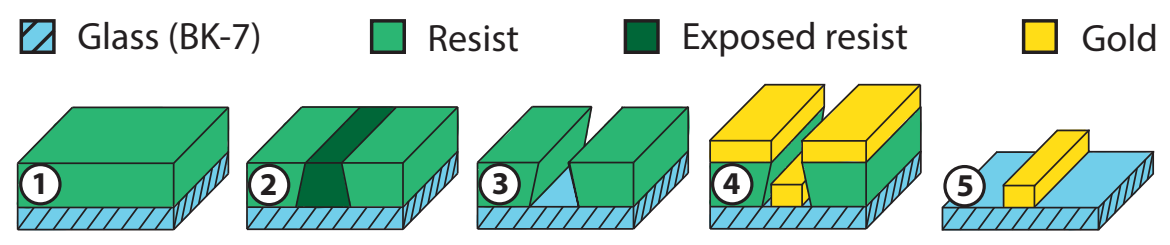

Figure A.1: E-beam lithography process flow. A cartoon depicting the main process steps which are performed during e-beam lithography fabrication. The consecutive steps involve: (1) spin coating the resist and grounding layer, (2) exposing the resist with an e-beam, (3) removing the exposed resist, (4) depositing a material on the patterned resist layer, and (5) finally removing the remaining resist.

and consequently the solubility of the exposed resist is increased. Selective removal of the exposed resist ('developing') is done by submerging the sample in a solvent ( $\mathrm{N}$-amyl acetate and MIBK:IPA 9:1) to stop development, leaving a trench in the resist (step 3).

The resolution one can acquire is determined by several factors, including resist thickness and acceleration voltage of the electron microscope. Also the temperature at which the resist is developed can have a large effect on the resolution. With today's electron optics, the beam waist of an electron beam can go down to a couple of $\mathrm{nm}$, which is mainly limited by the aberrations and space-charge effects. So the feature resolution will not be limited by the beam waist, but rather by the forward scattering of the electrons in the resist [167]. The forward scattering can be decreased by using higher energy electrons or a thinner resist. Another parameter which affects the obtained spatial resolution is the temperature of the solvent during the development step, which affects the overall solubility of the exposed resist. Because the focus of the electron beam profile is Gaussian in shape, tuning the threshold of the electron dose (on the Gaussian beam profile) at which the resist is developed, provides an extra parameter for tuning the minimum feature size. A resist thickness of $120 \mathrm{~nm}$ and a development temperature of -20 $C^{o}$ was used in the fabrication of the bar-ring antennas. A conductive polymer (E-spacer) was used to form the conductive layer on top of the resist, which acts as a ground plane to remove the excess charge. A key feature of this conductive polymer is that it is easily removed when rinsed with water, with minimal damage to the exposed resist layer; this in strong contrast to conventional methods using conducting metals.

A material of choice, in our case gold, is then deposited onto the designed 
mask by means of thermal evaporation techniques (step 4). As this is done in a large vacuum chamber under high vacuum conditions $(\mathrm{P}<2 \mathrm{e}-$ 6 mbar), the expanding cloud of evaporated material does not undergo any scattering, meaning that the angular distribution of the expanding cloud can be described as a sphere with increasing radii. The sample is positioned above the crucible at a distance of $\pm 40 \mathrm{~cm}$, allowing the spherical beam to be approximated by a flat beam front. To ensure good adhesion to the substrate surface, a short $\mathrm{O}_{2}$ plasma cleaning step was made and a $2 \mathrm{~nm}$ chromium layer was applied before evaporating the gold. In the last step, the resist layer is removed in an acetone bath, leaving the designed structure (step 5).

\section{A.2 Synthesis of single crystalline Au flakes}

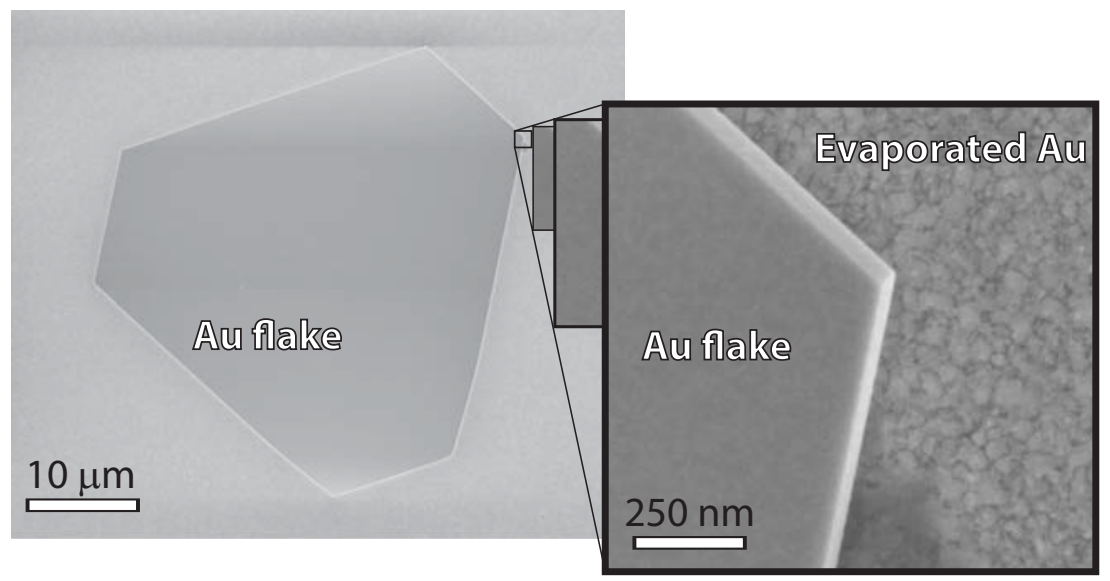

Figure A.2: Single crystalline gold flake. SEM micrograph of a single crystalline gold flake on a polycrystalline gold substrate.

For the fabrication of the highly detailed structures of Chapter 3, single crystalline gold flakes were used as a starting point for focused ion beam (FIB) patterning. The use of single crystalline gold flakes has been introduced to the optics community by Huang et al. [168], showing the increased resolution which can be obtained. The resolution of FIB fabrication techniques has been plagued by the limiting factor of the polycrystalline nature of many often used substrate materials. Gold substrates made by thermal evaporation techniques show grain sizes in the order of $50-100 \mathrm{~nm}$, which depends on the temperature of the substrate during the evaporation process. Using the single crystalline material as 
a fabrication starting point shows two main benefits. First, a higher fabrication resolution can be obtained [168]. Second, it has been shown that due to the absence of grain boundaries, structures made from this material show lower losses [169]. The chemical synthesis of single crystalline gold flakes, and the use of it as a substrate for fabricating high resolution nanostructures, has now been widely adopted $[168,170,171]$. Figure A.2 shows SEM micrographs of a single crystalline gold flake on a thermally evaporated gold substrate. The polycrystalline nature of the substrate is clearly visible in the image on the right.

In 2006 the synthesis of single crystalline gold flakes was already widely studied, although it had not yet been recognized that single crystalline gold flakes held an interesting application as fabrication substrates [172-174]. While there is still some debate on the growth mechanism, the standard explanation for the formation of the gold flakes involves the role of a performed 'seed', and a capping agent that controls the growth rate of various crystal facets of the seed. The stronger adsorption of capping agents on the (111) plane of Au nuclei accounts for the anisotropic growth of gold flakes.

However, Ref. [172] emphasizes that "the growth rate of crystals are often controlled (kinetically rather than thermodynamically) by the sticking probability on a given face. Since the (111) face of fcc metal has the lowest sticking probability being of lowest surface energy compared to other faces $\left(\gamma_{(110)}>\gamma_{(100)}>\gamma_{(111)}\right)$, the fcc metal confers its tendency to nucleate and grow into nanoparticles with their surfaces bounded by (111) facets, particularly in the process of chemical synthesis. Accordingly, it is reasonable to speculate that Au nuclei formed in the initial stage are kinetic structures with lowest energy (111) surfaces. Then at a favorite PVP/Au molar ratio, the polar groups, such as pyrrole rings of PVP and hydroxyl groups of ethylene glycol molecules, adsorb preferentially on the sites of the (111) planes of Au nuclei and interact with the (111) planes, which also obviously slows (or prevents) the growth on the (111) plane and promotes a highly anisotropic crystal growth, such as along (110) orientation, extending into large flakes with basal (111) planes. However, less or excessive PVP coverage on the surfaces of Au nanoparticles would induce a simultaneous growth for different crystal faces to some extend (or even growth limitation) apart from the anisotropic growth within the (111) plane, leading to the formation of multishaped of Au nanostructures."

The recipe of [172] was used for the fabrication of gold flakes in this thesis. Reproducing their result, however, can be quite challenging as 
the growth process is very sensitive to the molar ratio of gold-bearing solution $\left(\mathrm{HAuCl}_{4}\right)$, capping agent (poly(vinylpyrrolidone) (PVP)) and in particular the mixing speed. Although the molar ratio is stated, the dimensions of the used flask and the stirring speed are not mentioned, which we found out to be of great influence on the size and even the shape of particles which are obtained, varying from hexagonal, trapezium, rods and small ball-like crystal shapes. We used a spherical flask with a diameter of $5 \mathrm{~cm}$, a stirring magnet of $1 \mathrm{~cm}$ length, rotation speed of $400 \mathrm{rpm}$ and a molar ratio of 25-30. The solution with gold flakes was cleaned by diluting it with $80 C^{\circ}$ water and centrifuging at $2500 \mathrm{rpm}$, after which the solution was pipetted. This step was repeated three times.

\section{A.3 Focused Ion Beam}
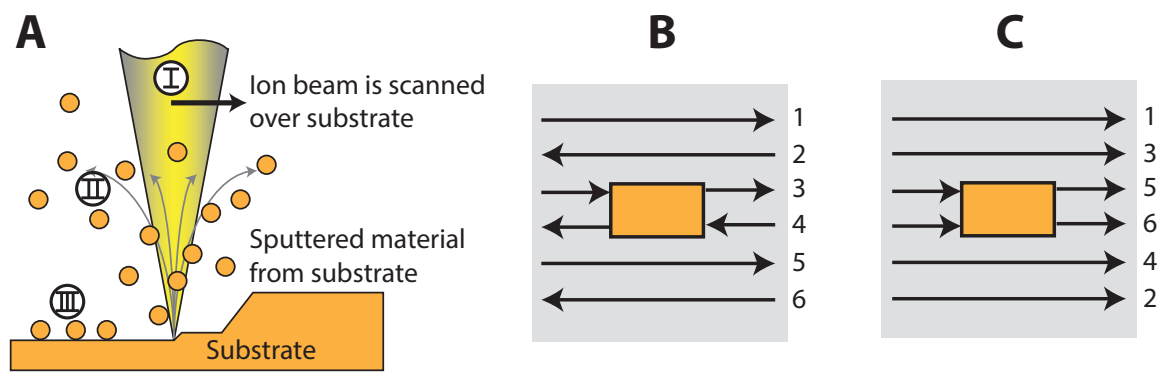

Figure A.3: Focused Ion Beam milling process and patterning schemes. (A) Cartoon depicting the FIB process, showing an ion-beam (1) which is focused on a substrate. Part of the material, which is removed from the substrate material is removed from the surface (2) and a part is redepsited (3). (B) Shows a standard patterning scheme often used in FIB fabrication. (C) Shows an optimized patterning scheme which reduces heat related fabrication defects.

A Focused Ion Beam (FIB), as a scientific instrument shows great similarities to the working principles of a scanning electron microscope (SEM), however instead of electrons a FIB uses gallium ions. Where the fabrication process using electron beam lithography consists of many steps, the advantage of FIB is that one can directly pattern a surface (see Figure A.3 A), and view the result. The removal of material in the FIB process can be nicely compared with the momentum transfer of two colliding billiard balls; the gallium ions when colliding with the substrate knock off atoms of the substrate (num. II in Figure A.3 A), and in the process some of the Gallium atoms are implanted into the substrate. Compared to the electrons in a SEM, Gallium ions have in the 
order of $10^{5}$ more mass. As a result of this, SEM fabrication techniques do not show this milling effect. Making high resolution nanostructures with FIB comes with its own challenges, which involve redeposition of previously milled material (III in Figure A.3A) and local heating. One can imagine that redeposition of milled material is undesirable, as it introduces a random factor in the process. The reason why local heating of the substrate can be a problem is maybe less obvious. Firstly local heating of the substrate increases the mobility of the atoms of the substrate and less energy is needed to remove them, thus reducing the effective dose needed. This effect often becomes apparent when the milling sequence follows a corner, where more material is removed as a result of local heating. Secondly when dealing with small structures and thus small volumes, strong heating of the local material will result in poor resolution, as the small volumes tend to reform to the most favorable thermodynamic shape, which often is a sphere-like shape. These effects

\section{Scanning electron micrographs}

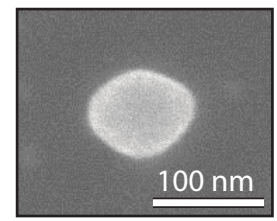

Standard patterning

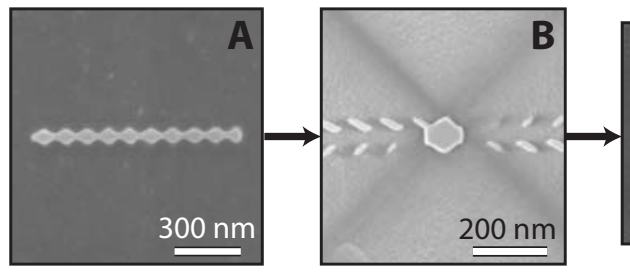

FIB - Scan patterns

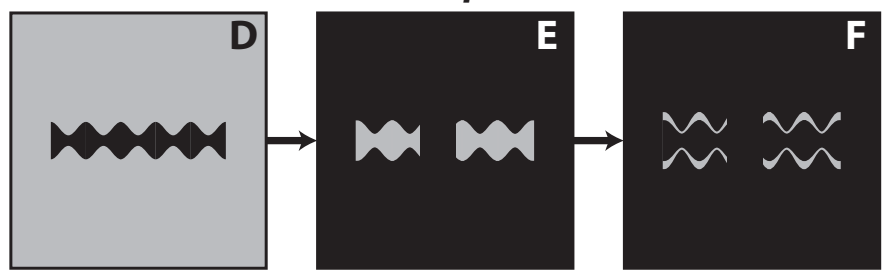

$\square=$ Patterned

Figure A.4: Fabrication of the modulated antennas. (Left) SEM micrograph of a nanostructure, made with a standard patterning scheme and to its right SEM micrographs of substructures (A-C), which are made during a three step FIB patterning, which are shown in (D-F). First a modulated rod of ten periods is made $(\mathbf{A}, \mathbf{D})$, after which the majority of the rod is removed, leaving a section of one period $(\mathbf{B}, \mathbf{E})$. An extra cleaning step is performed, which removes redeposited material near the structure $(\mathbf{C}, \mathbf{F})$.

can often be tackled by adapting the order of the FIB pattern (shown in Figure A.3 B and C). In pattern B the final antenna encounters a lot of heat from one direction, where pattern $\mathrm{C}$ introduces a more homogenous heating, which results in higher resolution structures and less 
redeposition as well.

The fabrication of the modulated antennas in Chapter 3 seems to suffer from both redeposition and heat effects. A typical structure using modified FIB patterning (similar to Figure A.3 C) is shown in the top left corner of Figure A.4. The end result is highly deformed with rounded end facets and low detail on the upper and lower surfaces. Both of which could be attributed to local heating effects. To address these effects, the more sophisticated three-step FIB pattern is applied. First a long sinusoidal modulated rod is made, which is shortened in the second step, and finally the residual material is removed. The final result shows a significant improvement compared to a structure made with modified FIB patterning. This three-step patterning process could possibly be used for the fabrication of many arbitrary shaped structures containing small features (down to $10 \mathrm{~nm}$ minimum feature size).

The fabrication of the hole structures shown in Chapters 5 and 6, predominantly suffers from redeposition effects, which becomes most apparent in the high aspect ratio holes of Chapter 5 . The designed cylindrical holes end up in a cone-like shape. Optimizing the FIB pattern hardly has an effect, as most of the material which is removed in the lowest part of the cone cannot escape. 
Appendix 1

\section{Details on numerical calculations}

For the numerical calculations of the fields, scattering spectra and the angle dependent scattering patterns, a commercial finite element method (FEM) package is used (CST microwave studio) [139]. In this appendix key details of the numerical calculations are listed.

\section{Illumination conditions}

If otherwise stated, a plane wave illumination is used which is orientated orthogonal to the structure top surface. In the scattering spectra shown in Figures 4.3 and 4.5 a plane wave illumination is used which is orientated under an angle $\alpha$. In all the numerical calculations shown in this thesis the illumination source is polarized parallel meaning that in Chapters 3 and 4 the polarization is set along the long axis of the bar antennas.

\section{Detection}

A far-field monitor is used for calculating the far-field scattering spectra. The far-field monitor detects the in- and outgoing power in a defined volume, which is measured for a distributions of angles. The polar plot shown in Figures 4.3 and 4.5 are obtained by calculating the scattering spectra when the structure is illuminated at a certain angle. The modulation of the resonance peaks in the scattering spectra as a function of excitation angle are used to compose the polar plot. 


\section{Substrate}

In the calculation of the scattering spectra and angle dependent scattering patterns no substrate of any kind are used. Instead the antennas are surrounded by a medium which has the average dielectric constant of the substrate and air. This is a method which is used more often and has proven to give reliable scattering spectra [175]. In the calculations of the angle dependent scattering patterns also no interface is used which will definitely affect the obtained scattering patterns however this is a deliberate choice as the scattering patterns are compared with a dipole model which also does not contain any interface (see Section 4.3).

\section{Dielectric constants}

For optical antennas the dielectric constants of gold are used which are obtained from the experimental data of Johnson an Christy [67], which are fitted with a 4th order polynomial.

\section{Meshing}

The meshing is set such that the smallest grid size is $2 \mathrm{~nm}$, after which an energy based optimization was applied. What this means is that mesh-size at the position of highest energy, largest field, is minimized, which is done in an iterative process. This iterative process is stopped when the obtained spectra do not change significantly with increasing the number of mesh-cells. 


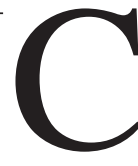

\section{Modified coupled harmonic oscillator model}

The coupled harmonic oscillator model is often used to describe the coupling of coupled optical antennas. In this appendix the mechanical analog of single harmonically driven oscillator is described after which the coupling of two oscillators having different polarizability and Qfactors is described.

\section{C.1 Single harmonically driven oscillator}

With the harmonic oscillator model we describe the movement of a mechanical oscillator, consisting out of a mass and spring, which is harmonically driven. We introduce two scaling parameters, where the first scales the magnitude of the mass, spring and damping constant $\left(R_{p}\right)$, and the second scales the $\mathrm{Q}$-factor $\left(R_{q}\right)$ of a mechanical oscillator. The resonance frequency, $\omega=\sqrt{\frac{k}{m}}$, in the loss-less harmonic oscillator system is determined by the ratio of its spring constant $(k)$ and mass $(m)$. If we assume a damped, harmonically-driven oscillator, the force balance takes the following form:

$$
\mathbf{R}_{p}\left(m \frac{d^{2} x}{d^{2} t}+k x+\mathbf{R}_{q} \gamma \frac{d x}{d t}\right)=F
$$

with $x(t)$ being the position of the mass as a function of time, $m$ the mass, $k$ spring constant, $\gamma$ the damping constant, and $F$ the driving force. Two extra parameters, $\mathbf{R}_{p}$ and $\mathbf{R}_{q}$, are introduced in the force balance, which are later shown to inversely scale with the polarizability and Q-factor of an optical antenna, respectively. Assuming the system is 
harmonically driven: $F=F_{0} e^{-i \omega t}$ and $x(t)=x_{0} e^{-i \omega t}$, the force balance can be rewritten to:

$$
\mathbf{R}_{p}\left(k-m \omega^{2}-i \mathbf{R}_{q} \omega \gamma\right) x=F
$$

The movement of the mass is then described as:

$$
x=\frac{F}{\mathbf{R}_{p}\left(k-m \omega^{2}-i \mathbf{R}_{q} \omega \gamma\right)}
$$

where $|x| \propto \frac{1}{\mathbf{R}_{p}}$, the amplitude of the mechanical oscillator inversely scales with $\mathbf{R}_{p}$. As an example, the amplitude of a mechanical oscillator with decreasing $\mathbf{R}_{p}$, and thus lower mass and spring constant, increases. Using the convention described above, the scaling of the Q-factor $(Q)$ is nicely described by the inverse scaling of $\mathbf{R}_{q}$ :

$$
Q=\frac{\sqrt{k m}}{\gamma} \Rightarrow \frac{\mathbf{R}_{p}}{\mathbf{R}_{p} \mathbf{R}_{q}} \frac{\sqrt{k m}}{\gamma} \Rightarrow Q \propto \frac{1}{\mathbf{R}_{q}}
$$

\section{C.2 The effects of loss}

By taking losses into account, a complete description of the resonant behavior of a mechanical oscillator can be found using the CHO model. First, we explore whether the absolute value of the Q-factors is important, or if just the Q-factor ratio of the two oscillators $\mathbf{R}_{q 1,2}$ is sufficient. Therefore, the absolute value of the Q-factor of oscillator one and a Qfactor ratio $\mathbf{R}_{q 1,2}$ are used, so that the effects of the overall loss of the system and the Q-factor ratio of the two oscillators can be explored. The eigenvalues and eigenvectors can be determined by solving the following force balance equation:

$$
\begin{aligned}
& \left(\begin{array}{cc}
m & 0 \\
0 & m \mathbf{R}_{p 1,2}^{\prime}
\end{array}\right)\left(\begin{array}{l}
\ddot{x}_{1}(t) \\
\ddot{x}_{2}(t)
\end{array}\right)+\left(\begin{array}{cc}
\gamma & 0 \\
0 & \mathbf{R}_{p 1,2}^{\prime} \mathbf{R}_{q 1,2} \gamma
\end{array}\right)\left(\begin{array}{l}
\dot{x}_{1}(t) \\
\dot{x}_{2}(t)
\end{array}\right) \\
& +\left(\begin{array}{cc}
k-k_{c} & k_{c} \\
k_{c} & \mathbf{R}_{p 1,2}^{\prime} k-k_{c}
\end{array}\right)\left(\begin{array}{l}
x_{1}(t) \\
x_{2}(t)
\end{array}\right)=0
\end{aligned}
$$

No simple analytical description of the eigenvalues and eigenvectors is obtained. In order to say more about the combined effect of $\mathbf{R}_{p 1,2}^{\prime}$ and $\mathbf{R}_{q 1,2}$, we numerically solve the absolute determinant of the force balance equation; Figure C.1 shows how this is done. The eigenvalues and eigenvectors of various two oscillator coupled systems, with constant $\mathbf{R}_{q 1,2}$ and $k_{c}$ values and varying values of $\mathbf{R}_{p 1,2}^{\prime}$, are calculated for various frequencies, see Figure C.1 A and B. The maximum values of the eigenvalue curves, indicated with gray dashed lines, are the eigenvalues of interest and indicate the resonance frequencies of the two eigenmodes 
of the coupled system, symmetric and anti-symmetric, which are plotted in green and blue for various values of $\mathbf{R}_{p 1,2}^{\prime}$. When losses are included

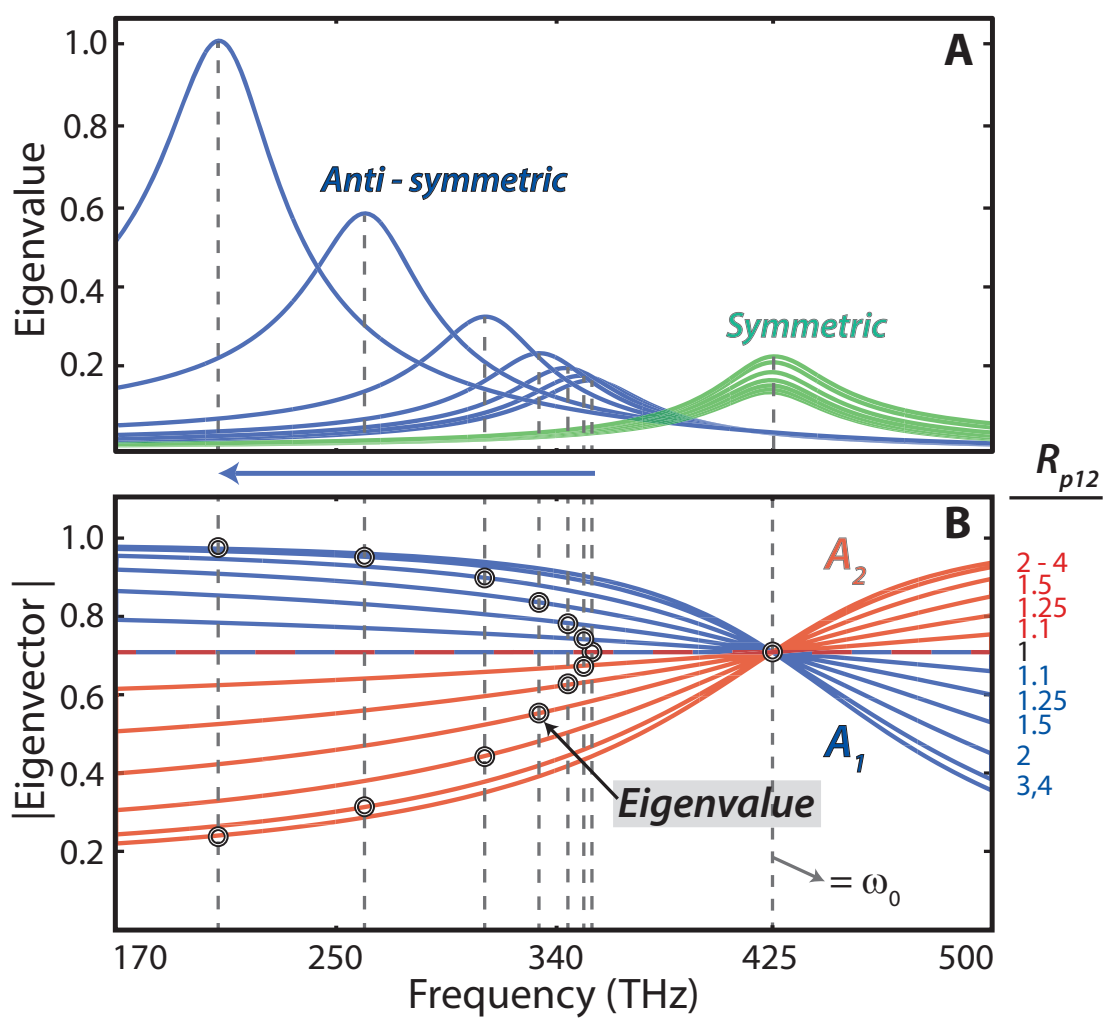

Figure C.1: Frequency dependent eigenvalue and eigenvector calculations. The eigenvalues $(\mathbf{A})$ and eigenvectors $(\mathbf{B})$ are calculated for various coupled harmonic oscillators with different polarizability ratios $\mathbf{R}_{p 1,2}^{\prime}$, with $\mathbf{R}_{q}=1$, and a coupling constant $k_{c}=0.15$. The green and blue curves in $\mathbf{A}$ represent the symmetric and anti symmetric eigenmodes of the coupled system, respectively. At the resonance frequency of the two eigenmodes of the coupled system, the corresponding eigenvector / amplitude of the individual mechanical oscillators, shown with red and blue lines, are indicated by black circles in B.

in the model, again we find that the resonance frequency of the symmetric eigenmode is almost constant, while the resonance frequency of the anti-symmetric eigenmode is strongly redshifting with increasing $\mathbf{R}_{p 1,2}^{\prime}$, indicated with the blue arrow in Figure C.1. The eigenvectors are calculated for various frequencies; the blue and red lines show the absolute amplitudes of the individual oscillators, $A_{1}$ and $A_{2}$, for various values of $\mathbf{R}_{p 1,2}$, indicated on the right of Figure C.1 B. Similar to derivation of the eigenvectors of a loss-less system, in Equation 4.4 , for a $\mathbf{R}_{p 1,2}^{\prime}$ ratio 
of one, the red and blue curve overlap, indicating that the amplitude ratio of the individual oscillators is equal to one for both the eigenmodes. For increasing $\mathbf{R}_{p 1,2}^{\prime}$ values, the amplitudes of the individual oscillators quickly diverge where $A_{1}>A_{2}$ and $A_{1}<A_{2}$, for frequencies respectively lower and higher than $\omega_{0}$. At the frequencies of the maximum eigenvalues, indicated with vertical gray dashed lines, the amplitudes of the individual oscillators are determined, indicated in Figure C.1 B with black circles. The red-shifting trend of the eigenvalues of the anti-symmetric eigenmodes goes hand-in-hand with an increasing dispersion of the amplitudes of the individual oscillators, for increasing $\mathbf{R}_{p 1,2}^{\prime}$. Because of the stationary nature of the eigenvalues of the symmetric eigenmode, the eigenvector ratios for different values of $\mathbf{R}_{p 1,2}^{\prime}$, are constant at a ratio of 1:1, similar to the found eigenvectors of a lossless system in Equation 4.4.

In order to obtain the full picture of the combined effects of $\mathbf{R}_{p 1,2}^{\prime}$ and $\mathbf{R}_{q 1,2}$, on the amplitude ratio of the individual oscillators, a $2 \mathrm{D}$ landscape of the amplitude ratio is shown in Figure C.2, where $\mathbf{R}_{p 1,2}^{\prime}$ and $\mathbf{R}_{q 1,2}$ are varied on either axis of the figure. Three different iterations are performed with different degrees of overall loss, where the ratio of the Q-factors $\left(R_{q 1,2}=q_{1} / q_{2}\right)$ are referenced to the Q-factor of oscillator one: $q_{1}=50,10$ and 5 . These values are chosen, as they incrementally become more realistic values for optical antennas, with $q_{1}=5$ being the most realistic. For the three different Q-factor iterations in Figure C.2, the amplitude ratios of the anti-symmetric eigenmode show to be unaffected by the Q-factor $\mathbf{R}_{q 1,2}$, and scale linearly with $\mathbf{R}_{p 1,2}^{\prime}$, resulting in predominant vertical contour lines. The three iterations for the symmetric eigenmode show a very flat $2 \mathrm{D}$ landscape, with an amplitude ratio of 1. Introducing more losses in the system, however, makes it increasingly difficult to determine two separate eigenvalues, resulting, at a certain point in a coalescence of the two. First signs of this process are seen for $q_{1}=10$ and 5 , where amplitude ratios smaller than one start to emerge. Increasing the coupling constant, and thereby increasing the separation of the eigenvalues negates, this effect and an amplitude ratio of 1 is again obtained. 


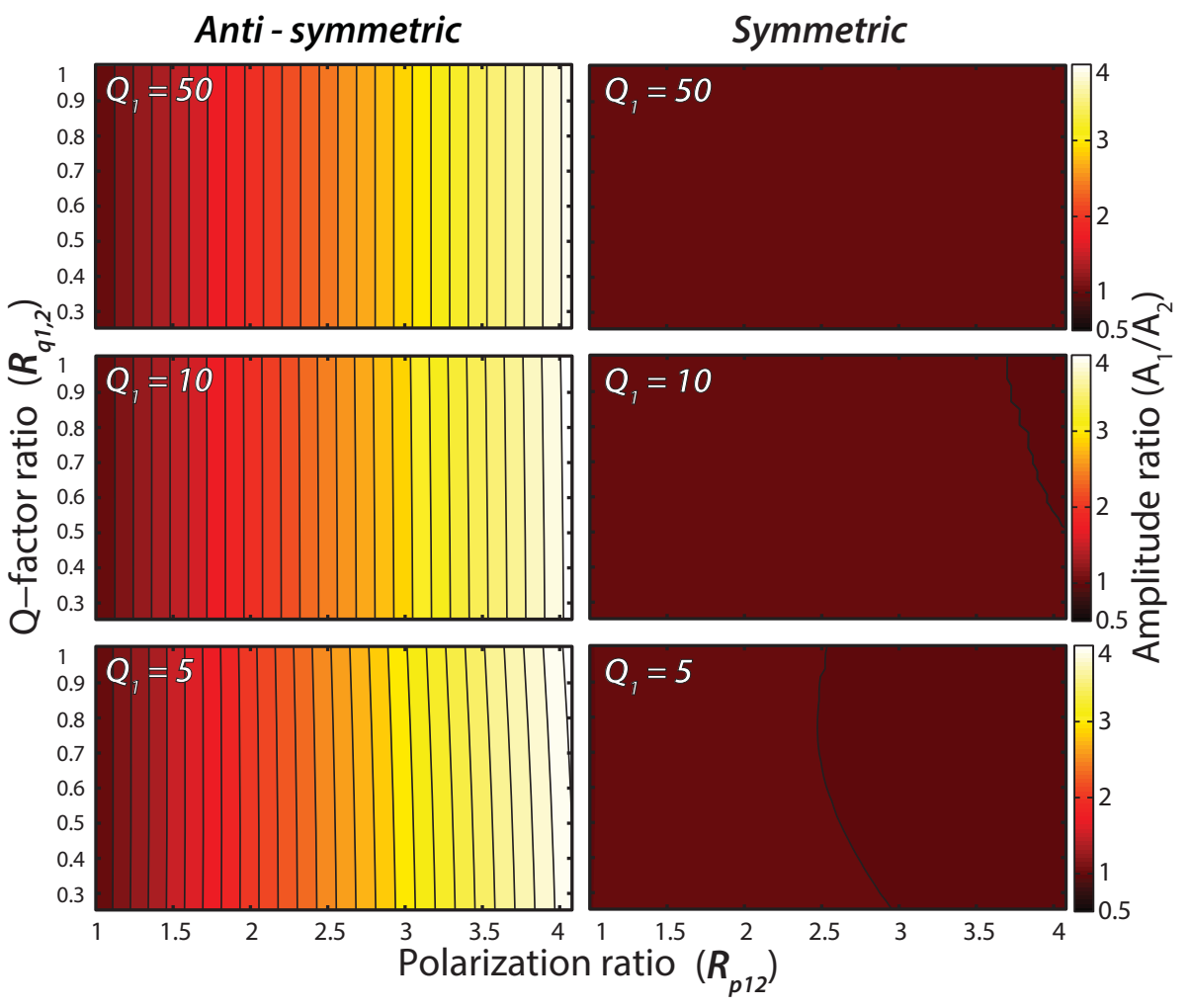

Figure C.2: Amplitude ratio calculations of two coupled optical resonators. Calculated amplitude ratios of two coupled optical resonators with different ratios of $\mathbf{R}_{p 1,2}^{\prime}$ and $\mathbf{R}_{q 1,2}$, represented in a $2 \mathrm{D}$ landscape. The amplitude ratios are calculated for both the symmetric and anti-symmetric eigenmodes in the case of relatively low (top) and highly (bottom) damped systems, with initial Q-factors of 50,10 and 5 .

In summary, the amplitude ratio of two coupled oscillators, with or without losses taken into account, is solely affected by the value of their $\mathbf{R}_{p 1,2}^{\prime}$. This behavior leads to the interesting result, where in the case of the symmetric eigenmode the amplitudes of both the oscillators are of equal magnitude. Coupling an oscillator which exhibits a low intrinsic amplitude with an oscillator with a high intrinsic amplitude, then lead to the interesting situation in which the amplitude of the oscillator with low intrinsic amplitude is enhanced with a factor $\mathbf{R}_{p 1,2}^{\prime}$. In Section 4.4 this hypothesis is tested for two optical antenna systems which show increasing degrees of complexity, see Figures 4.10 and 4.11. 
Appendix

\section{Reciprocity theorem}

Although great progress has been made in the field of near-field microscopy, for a long time only two EM near-field components could be measured simultaneously $[78,91,176,177]$. Similar near-field microscope setups as the one used in this thesis, had only been able to measure the in-plane electric field components $E_{x}$ and $E_{y}$, though it was shown that if sufficient prior knowledge is available, the in-plane electric fields are sufficient to construct the magnetic field components [176]. Recently, Le Feber et al. have made a big step forward by showing the direct mapping of all in-plane electromagnetic near-fields with a conventional, symmetric aperture probe [129]. They showed that by using the optical reciprocity theorem they could describe the fields above a photonic crystal waveguide in terms of the in-plane electric $\left(E_{x, y}\right)$ and magnetic $\left(H_{x, y}\right)$ components. Using the same optical reciprocity theorem the experimental field, measured with an aperture-less near-field microscope containing both electric and magnetic components, could also be described accurately [178].

The natural question that arises from their work is how the measured signal in our experiments relates to the EM fields present, therefore in this appendix we briefly show how the complex expression for the detected signal: $L_{(x, y)}=\eta_{e} E_{(x, y)}+\eta_{h} H_{(y, x)}$ is derived by applying the optical reciprocity theorem on the aperture near-field probe, following the method described in $[129,151,179]$. Here $\eta_{e}$ and $\eta_{h}$ describe the sensitivity of the aperture probe for the electric and magnetic fields.

In short the optical reciprocity theorem is a method to relate the fields emitted from a source, and the resulting fields impinging on a detector. The coupling strength between a source and a detector, also known as the mutual impedance, is found by calculating the overlap 


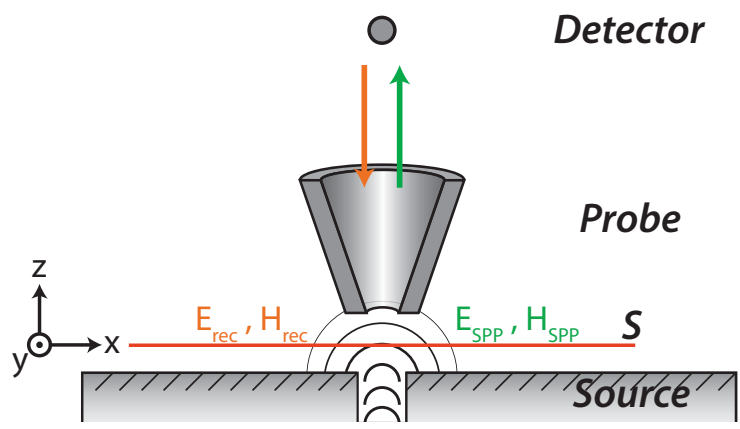

Figure D.1: Cartoon depicting the optical reciprocity theorem applied to a near-field probe. The gray surface represents a metallic surface with an aperture SPP source. The green and orange arrows and text indicate the experimental and reciprocal fields. The reciprocal fields arise from a dipole $\left(\mathbf{j}_{r e c}\right)$, which is placed at the position of the detector. The SPP and reciprocal fields on the surface $\mathrm{S}$ are indicated with $\mathbf{E}_{S P P, r e c}$ and $\mathbf{H}_{S P P, r e c}$, in green and orange, respectively.

integral of two sources positioned at the location of the source and the detector, see fig. D.1. The overlap integral is calculated over a surface $\mathrm{S}$, which in this case is positioned at a small distance underneath the aperture probe [179]. The fields on $\mathrm{S}$ represent the local fields which are generated by the source. In the case of a near-field microscope, the coupling between the source and the detector is governed by the aperture probe. By calculating the mutual impedance, the sensitivity of the aperture probe for the electric and magnetic fields can be derived. Figure D.1 shows a cartoon of an aperture probe above a metallic surface with an aperture SPP source, similar to the kind of structures studied in Chapters 5 and 6 . The electromagnetic fields associated with light propagating along the dielectric-metal interface are $\mathbf{E}_{S P P}$ and $\mathbf{H}_{S P P}$. The detected signal $\mathbf{E}_{\text {det }}$ is the result of the interaction of the near-field probe with these fields. The reciprocal fields, $\mathbf{E}_{r e c}$ and $\mathbf{H}_{r e c}$ are formed by placing a dipole source $\mathbf{j}_{\text {rec }}$ at the position of the detector. The mutual impedance of our system is described as $[129,179]$ :

$$
\mathbf{E}_{d e t} \cdot \mathbf{j}_{r e c}=\int_{S}\left(\mathbf{E}_{S P P} \times \mathbf{H}_{r e c}\right)-\left(\mathbf{E}_{r e c} \times \mathbf{H}_{S P P}\right) \cdot \mathbf{d} \mathbf{S}
$$

and

$$
\begin{array}{r}
\mathbf{E}_{\text {det }} \cdot \mathbf{j}_{r e c}=\int_{S}\left(E_{x, S P P} H_{y, r e c}-E_{y, S P P} H_{x, r e c}\right)- \\
\left(E_{y, r e c} H_{x, S P P}-E_{x, r e c} H_{y, S P P}\right) d S
\end{array}
$$

Equation D.1 shows that the sensitivity of experimental electric field $\left(E_{S P P}\right)$ is governed by the magnetic component of the reciprocal field 
and vice versa. The surface $S$ is chosen in the $x y$-plane [129], which is a natural choice as most of the near-field measurements are performed in the $x y$-plane. Because the dot product is taken of dS, which is orientated along $\hat{\mathbf{z}}$, with the cross product in the integral, only the in-plane reciprocal and SPP fields contribute to the mutual impedance. Equation D.2 shows that potentially eight different fields contribute to the signal which is measured at the detector. In order to understand how the different fields will contribute to the experimental signal, we numerically calculate the EM field distribution of an aluminum film with an aperture with a $125 \mathrm{~nm}$ radius, excited at $950 \mathrm{~nm}$ (see fig. D.2). The field

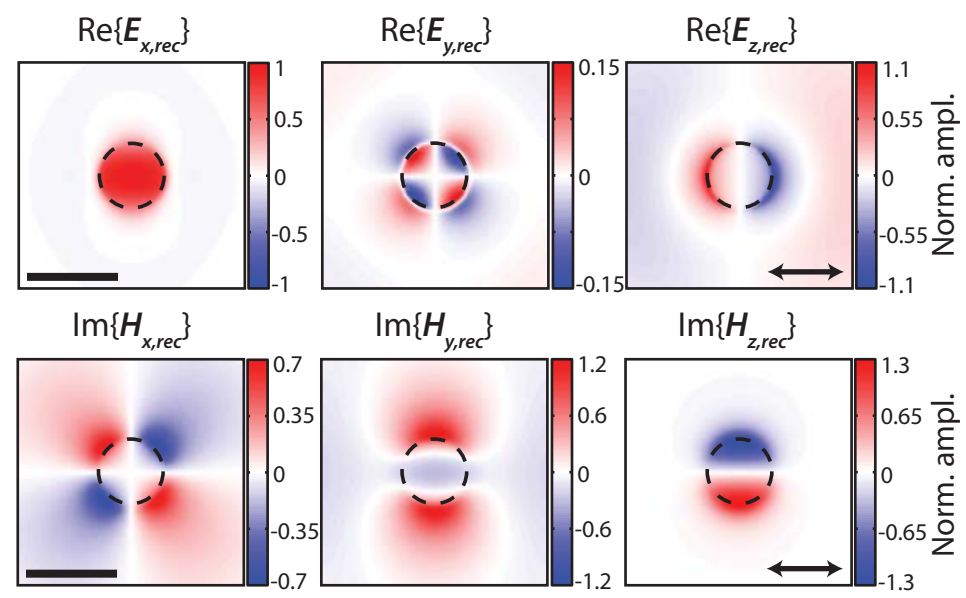

Figure D.2: Electric and magnetic field distribution of a single hole. Numerical calculations of the EM field distribution, $10 \mathrm{~nm}$ above the surface of a single hole with a radius of $125 \mathrm{~nm}$, made in an aluminum film. The hole is excited with horizontal polarized light (with $\lambda_{\text {res }}=950 \mathrm{~nm}$ ), indicated with the black arrow. The amplitudes are normalized to the maximum value of $E_{x, r e c}$. The black bar indicates a length of $400 \mathrm{~nm}$.

distribution of a hole in an aluminum film is very similar to that of a hole in a gold film (see Figure 5.6). However, they show two main differences: first the field distributions of the aluminum film show a stronger localization to the hole and less surface plasmons are generated. Second, the magnitude difference between $E_{x}$ and $E_{y}$ is larger in the case of the aluminum film compared to an aperture in a gold film. The sensitivity of $\mathbf{L}_{x}$ will be determined by the field distributions of fig. D.2 and the respective ratio of their magnitudes. Based on equation D.2, combined with the fact that fig. D.2, shows that $E_{x, r e c}$ is much larger than $E_{y, r e c}$, we expect that the magnetic contribution to $\mathbf{L}_{x}$ will be dominated by $H_{y, S P P}$. 
Determining the dominant electric contribution to $\mathbf{L}_{x}$ is less straightforward, as the in-plane magnetic reciprocal fields are of almost equal magnitude. However, in the limit of an aperture probe being much smaller than the wavelength, to the first order approximation, the sum over the fields rather than the field distribution can be used to find the sensitivity [151]. Because the sum over $H_{x, \text { rec }}$ is very small, it is expected that the $E_{y, S P P}$ component will make up a small portion of the total signal. The experimental signals can thus be described as being a combination of an electric and a magnetic component:

$$
\begin{aligned}
& \mathbf{L}_{x}=\eta_{E} \mathbf{E}_{x}(r)+\eta_{H} \mathbf{H}_{y}(r) \\
& \mathbf{L}_{y}=\eta_{E} \mathbf{E}_{y}(r)-\eta_{H} \mathbf{H}_{x}(r)
\end{aligned}
$$

where $\eta_{E}$ and $\eta_{H}$ describe the sensitivity of the system to the electric and magnetic in-plane fields. 
Appendix

\section{Field distributions of holes with increasing diameter}

In this appendix the experimental field distributions $\mathbf{L}_{(x, y)}$ of a series of holes having a diameter varying from 100 to $600 \mathrm{~nm}$, are shown. The holes are made in a $250 \mathrm{~nm}$ thick gold film on a glass substrate and are excited with horizontally polarized light. In Figures E.1 and E.2 the absolute (top panel) and real components (center) are shown together with the two dimensional Fourier transform (bottom). The semi circular peaks seen in the two k-vector distributions of $\mathbf{L}_{x}$ at $\left|K_{(x, y)}\right|=1$, indicate the presence of surface plasmon polaritons (SPPs) propagating in the $\mathrm{x}$ and -x direction. Similarly the four lobes in the k-vector distribution of $\mathbf{L}_{y}$ indicate the propagation direction of the outward propagating SPP. The k-vector distribution in the range of $0<\left|k_{(x, y)}\right|<1$ is associated with the free-space emission of the hole. As the hole diameter increases the k-vector distributions of $\mathbf{L}_{(x, y)}$ become more localized towards $\left|k_{(x, y)}\right|<1$, indicating that the free-space emission of the holes becomes more directional. The ratio of the free-space emission and the excitation of SPPs as a function of hole diameter is discussed in Chapter 5. 


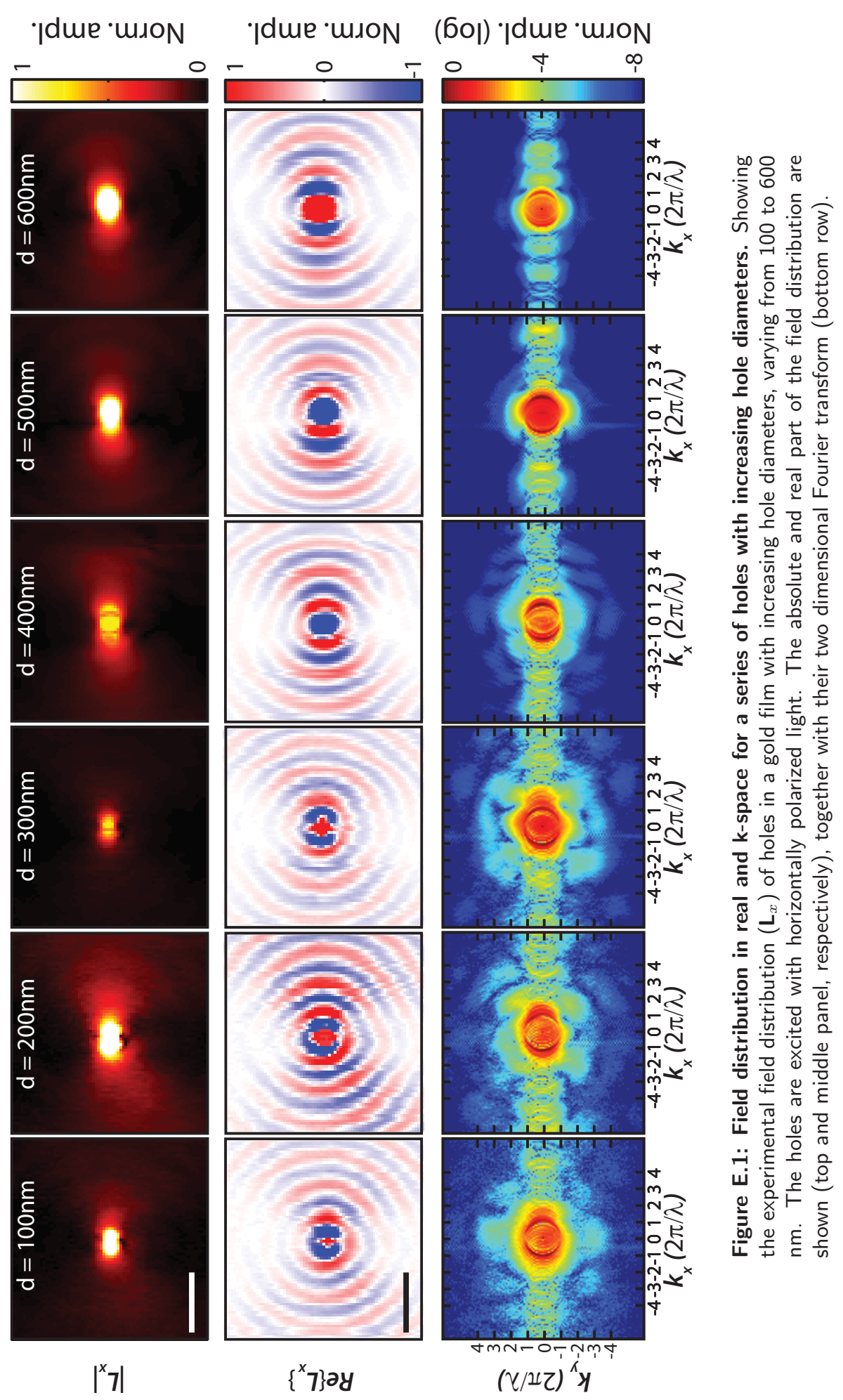




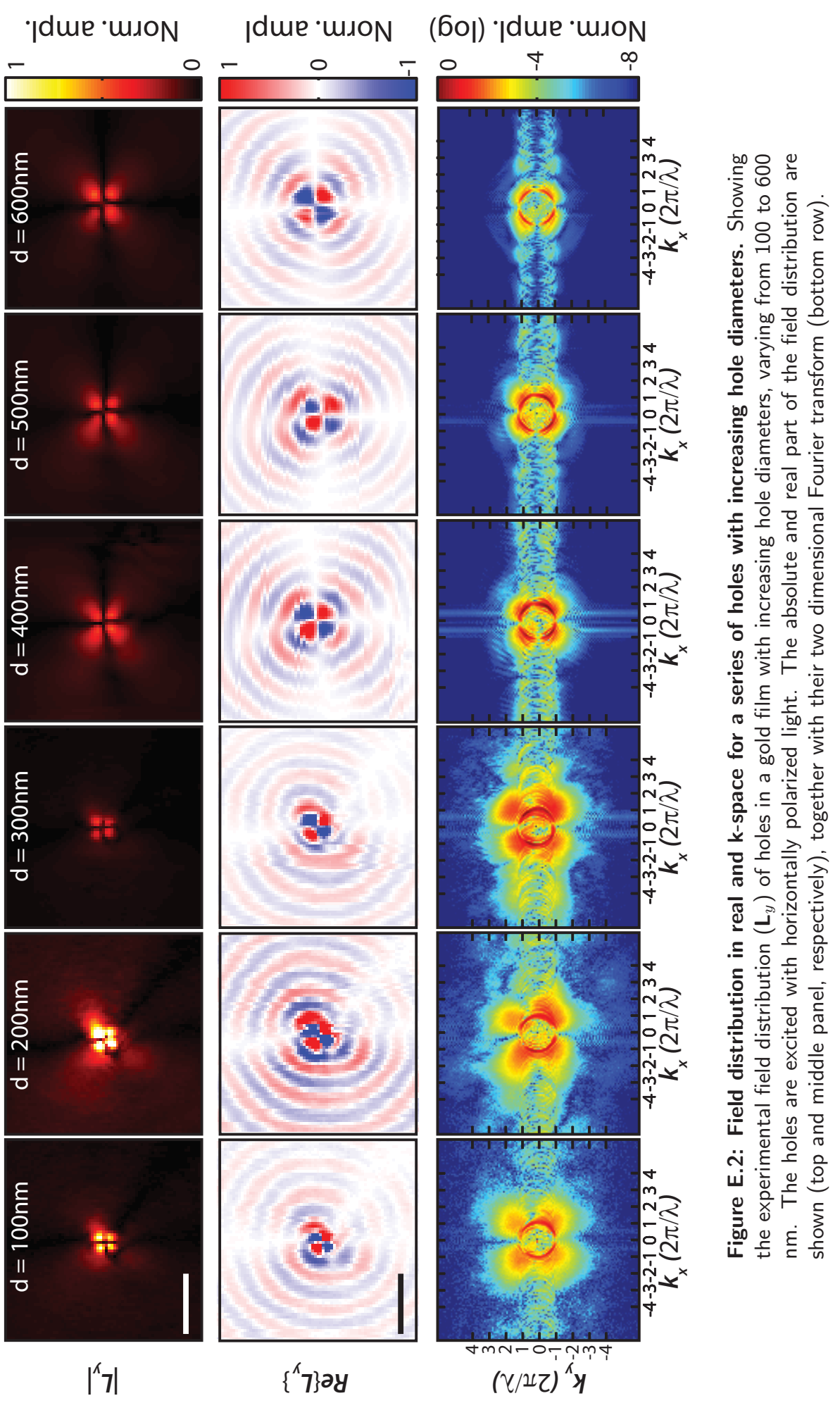




\section{Bibliography}

[1] P. Bharadwaj, B. Deutsch, and L. Novotny, "Optical Antennas," Advances in Optics and Photonics 1, 438 (2009).

[2] M. L. Brongersma, N. J. Halas, and P. Nordlander, "Plasmon-induced hot carrier science and technology," Nature Nanotechnology 10, 25-34 (2015).

[3] S. V. Boriskina, H. Ghasemi, and G. Chen, "Plasmonic materials for energy : From physics to applications," Biochemical Pharmacology 16, 375-386 (2013).

[4] X. Fan, W. Zheng, and D. J. Singh, "Light scattering and surface plasmons on small spherical particles," Light Sci Appl 3, e179 (2014).

[5] D. K. Gramotnev and S. I. Bozhevolnyi, "Nanofocusing of electromagnetic radiation." Nature Photonics 8, 13-22 (2013).

[6] H. A. Atwater and A. Polman, "Plasmonics for improved photovoltaic devices." Nature materials 9, 205-213 (2010).

[7] J. N. Anker, W. P. Hall, O. Lyandres, N. C. Shah, J. Zhao, and R. P. Van Duyne, "Biosensing with plasmonic nanosensors," Nat Mater 7, 442-453 (2008).

[8] N. Liu, M. L. Tang, M. Hentschel, H. Giessen, and a. P. Alivisatos, "Nanoantenna-enhanced gas sensing in a single tailored nanofocus." Nature materials 10, 631-636 (2011).

[9] A. F. Coskun, A. E. Cetin, B. C. Galarreta, D. A. Alvarez, H. Altug, and A. Ozcan, "Lensfree optofluidic plasmonic sensor for real-time and label-free monitoring of molecular binding events over a wide field-of-view," Sci. Rep. 4 (2014).

[10] J. Jackson, Classical Electrodynamics (John Wiley \& Sons, 1962).

[11] T. Zentgraf, T. P. Meyrath, A. Seidel, S. Kaiser, H. Giessen, C. Rockstuhl, and F. Lederer, "Babinet's principle for optical frequency metamaterials and nanoantennas," Phys. Rev. B 76, 033407 (2007).

[12] S. Koo, M. S. Kumar, J. Shin, D. Kim, and N. Park, "Extraordinary Magnetic Field Enhancement with Metallic Nanowire: Role of Surface Impedance in Babinet's Principle for Sub-Skin-Depth Regime," Phys. Rev. Lett. 103, 263901 (2009).

[13] B. Oeguet, R. Vogelgesang, W. Sigle, N. Talebi, C. T. Koch, and P. A. van Aken, "Hybridized Metal Slit Eigenmodes as an Illustration of Babinet's Principle," ACS NANO 5, 6701-6706 (2011). 
[14] M. Li, S. K. Cushing, and N. Wu, "Plasmon-enhanced optical sensors: a review," Analyst 140, 386-406 (2014).

[15] J. A. Schuller, E. S. Barnard, W. Cai, Y. C. Jun, J. S. White, and M. L. Brongersma, "Plasmonics for extreme light concentration and manipulation," Nat Mater 9, 193-204 (2010).

[16] N. Gao, K. Huang, J. Li, S. Li, X. Yang, and J. Kang, "Surface-plasmonenhanced deep-UV light emitting diodes based on AlGaN multi-quantum wells," Sci. Rep. 2 (2012).

[17] E. Ozbay, "Plasmonics: Merging Photonics and Electronics at Nanoscale Dimensions," Science 311, 189-193 (2006).

[18] N. Engheta, "Circuits with Light at Nanoscales: Optical Nanocircuits Inspired by Metamaterials," Science 317, 1698-1702 (2007).

[19] H. Caglayan, S.-H. Hong, B. Edwards, C. R. Kagan, and N. Engheta, "NearInfrared Metatronic Nanocircuits by Design," Phys. Rev. Lett. 111, 073904 (2013).

[20] L. Novotny and N. van Hulst, "Antennas for light," Nat Photon 5, 83-90 (2011).

[21] L. Novotny, "The history of near-field optics," in "Progress in Optics," , vol. 50, E. Wolf, ed. (Elsevier, 2007), pp. 137-184.

[22] G. W. Bryant, F. J. Garca de Abajo, and J. Aizpurua, "Mapping the Plasmon Resonances of Metallic Nanoantennas," Nano Lett. 8, 631-636 (2008).

[23] L. Novotny and B. Hecht, Principles of nano-optics (Cambridge, university press, Cambridge, 2008), 1st ed.

[24] K. Li, M. I. Stockman, and D. J. Bergman, "Self-Similar Chain of Metal Nanospheres as an Efficient Nanolens," Phys. Rev. Lett. 91, 227402 (2003).

[25] M. Schnell, P. Alonso-Gonzlez, L. Arzubiaga, F. Casanova, L. E. Hueso, A. Chuvilin, and R. Hillenbrand, "Nanofocusing of mid-infrared energy with tapered transmission lines," Nat Photon 5, 283-287 (2011).

[26] T. Hanke, G. Krauss, D. Trutlein, B. Wild, R. Bratschitsch, and A. Leitenstorfer, "Efficient Nonlinear Light Emission of Single Gold Optical Antennas Driven by Few-Cycle Near-Infrared Pulses," Phys. Rev. Lett. 103, 257404 (2009).

[27] J. Renger, R. Quidant, N. van Hulst, and L. Novotny, "Surface-Enhanced Nonlinear Four-Wave Mixing," Phys. Rev. Lett. 104, 046803 (2010).

[28] M. Kauranen and A. V. Zayats, "Nonlinear plasmonics," Nat Photon 6, 737748 (2012).

[29] H. Harutyunyan, G. Volpe, R. Quidant, and L. Novotny, "Enhancing the Nonlinear Optical Response Using Multifrequency Gold-Nanowire Antennas," Phys. Rev. Lett. 108, 217403 (2012).

[30] S. B. Hasan, F. Lederer, and C. Rockstuhl, "Nonlinear plasmonic antennas," Materials Today 17, 478-485 (2014).

[31] M. Celebrano, X. Wu, M. Baselli, S. Gromann, P. Biagioni, A. Locatelli, C. De Angelis, G. Cerullo, R. Osellame, B. Hecht, L. Du, F. Ciccacci, and M. Finazzi, "Mode matching in multiresonant plasmonic nanoantennas for enhanced second harmonic generation," Nat Nano 10, 412-417 (2015). 
[32] N. Yu, P. Genevet, M. A. Kats, F. Aieta, J.-P. Tetienne, F. Capasso, and Z. Gaburro, "Light Propagation with Phase Discontinuities: Generalized Laws of Reflection and Refraction," Science 334, 333-337 (2011).

[33] J. J. Mock, M. Barbic, D. R. Smith, D. A. Schultz, and S. Schultz, "Shape effects in plasmon resonance of individual colloidal silver nanoparticles," The Journal of Chemical Physics 116, 6755-6759 (2002).

[34] H. Kuwata, H. Tamaru, K. Esumi, and K. Miyano, "Resonant light scattering from metal nanoparticles: Practical analysis beyond Rayleigh approximation," 83, 4625 (2003).

[35] V. Giannini, A. I. Fernndez-Domnguez, S. C. Heck, and S. A. Maier, "Plasmonic Nanoantennas: Fundamentals and Their Use in Controlling the Radiative Properties of Nanoemitters," Chem. Rev. 111, 3888-3912 (2011).

[36] N. Berkovitch, P. Ginzburg, and M. Orenstein, "Nano-plasmonic antennas in the near infrared regime," Journal of Physics: Condensed Matter 24, 073202 (2012).

[37] C. F. Bohren and D. R. Huffman, "Absorption cross-section maxima and minima in IR absorption bands of small ionic ellipsoidal particles," Appl Opt 20, 959-962 (1981).

[38] A. Moroz, "Depolarization field of spheroidal particles," Journal of the Optical Society of America B 26, 517 (2009).

[39] T. Coenen, F. Bernal Arango, A. Femius Koenderink, and A. Polman, "Directional emission from a single plasmonic scatterer," Nat Commun 5 (2014).

[40] Y. Alaverdyan, N. Vamivakas, J. Barnes, C. Lebouteiller, J. Hare, and M. Atatre, "Spectral tunability of a plasmonic antenna with a dielectric nanocrystal," Optics Express 19, 18175 (2011).

[41] E. Massa, S. a. Maier, and V. Giannini, "An analytical approach to light scattering from small cubic and rectangular cuboidal nanoantennas," New Journal of Physics 15 (2013).

[42] H. Chen, L. Shao, Q. Li, and J. Wang, "Gold nanorods and their plasmonic properties," Chem. Soc. Rev. 42, 2679-2724 (2013).

[43] L. Feng, D. Van Orden, M. Abashin, Q.-J. Wang, Y.-F. Chen, V. Lomakin, and Y. Fainman, "Nanoscale optical field localization by resonantly focused plasmons," Optics Express 17, 4824 (2009).

[44] T. Zhang, G. Lu, H. Shen, K. Shi, Y. Jiang, D. Xu, and Q. Gong, "Photoluminescence of a single complex plasmonic nanoparticle," Sci. Rep. 4 (2014).

[45] J. Butet, K. Thyagarajan, and O. J. F. Martin, "Ultrasensitive optical shape characterization of gold nanoantennas using second harmonic generation," Nano Letters 13, 1787-1792 (2013).

[46] V. Myroshnychenko, J. Rodrguez-Fernndez, I. Pastoriza-Santos, A. M. Funston, C. Novo, P. Mulvaney, L. M. Liz-Marzn, and F. J. G. d. Abajo, "Modelling the optical response of gold nanoparticles," Chem. Soc. Rev. 37, 1792-1805 (2008).

[47] V. Myroshnychenko, E. Carb-Argibay, I. Pastoriza-Santos, J. Prez-Juste, L. M. Liz-Marzn, and F. J. Garca de Abajo, "Modeling the Optical Response of Highly Faceted Metal Nanoparticles with a Fully 3d Boundary Element Method," Adv. Mater. 20, 4288-4293 (2008). 
[48] P. Biagioni, J.-S. Huang, and B. Hecht, "Nanoantennas for visible and infrared radiation," Reports on Progress in Physics 75, 024402 (2012).

[49] L. Novotny, "Effective Wavelength Scaling for Optical Antennas," 266802, 1-4 (2007).

[50] S. A. Maier, "Effective Mode Volume of Nanoscale Plasmon Cavities," Opt Quant Electron 38, 257-267 (2006).

[51] E. Feigenbaum and M. Orenstein, "Ultrasmall Volume Plasmons, yet with Complete Retardation Effects," Phys. Rev. Lett. 101, 163902 (2008).

[52] A. M. Funston, C. Novo, T. J. Davis, and P. Mulvaney, "Plasmon Coupling of Gold Nanorods at Short Distances and in Different Geometries," Nano Lett. 9, 1651-1658 (2009).

[53] H. Wang and N. J. Halas, "Plasmonic Nanoparticle Heterodimers in a Semiembedded Geometry Fabricated by Stepwise Upright Assembly," Nano Lett. 6, 2945-2948 (2006).

[54] F. Hao, Y. Sonnefraud, P. V. Dorpe, S. A. Maier, N. J. Halas, and P. Nordlander, "Symmetry Breaking in Plasmonic Nanocavities: Subradiant LSPR Sensing and a Tunable Fano Resonance," Nano Lett. 8, 3983-3988 (2008).

[55] J.-S. Huang, J. Kern, P. Geisler, P. Weinmann, M. Kamp, A. Forchel, P. Biagioni, and B. Hecht, "Mode Imaging and Selection in Strongly Coupled Nanoantennas," Nano Lett. 10, 2105-2110 (2010).

[56] N. Papasimakis, V. A. Fedotov, N. I. Zheludev, and S. L. Prosvirnin, "Metamaterial Analog of Electromagnetically Induced Transparency," Phys. Rev. Lett. 101, 253903 (2008).

[57] S. Zhang, D. A. Genov, Y. Wang, M. Liu, and X. Zhang, "Plasmon-Induced Transparency in Metamaterials," Phys. Rev. Lett. 101, 047401 (2008).

[58] N. Liu, L. Langguth, T. Weiss, J. Kstel, M. Fleischhauer, T. Pfau, and H. Giessen, "Plasmonic analogue of electromagnetically induced transparency at the Drude damping limit," Nat Mater 8, 758-762 (2009).

[59] L. Novotny, "Strong coupling, energy splitting, and level crossings: A classical perspective," American Journal of Physics 78, 1199-1202 (2010).

[60] C. L. G. Alzar, M. a. G. Martinez, and P. Nussenzveig, "Classical analog of electromagnetically induced transparency," American Journal of Physics 70, 37-41 (2002).

[61] E. Prodan, C. Radloff, N. J. Halas, and P. Nordlander, "A Hybridization Model for the Plasmon Response of Complex Nanostructures," Science 302, 419-422 (2003).

[62] F. Lpez-Tejeira, S. G. Rodrigo, L. Martn-Moreno, F. J. Garca-Vidal, E. Devaux, T. W. Ebbesen, J. R. Krenn, I. P. Radko, S. I. Bozhevolnyi, M. U. Gonzlez, J. C. Weeber, and A. Dereux, "Efficient unidirectional nanoslit couplers for surface plasmons," Nat Phys 3, 324-328 (2007).

[63] Y. Alaverdyan, B. Seplveda, L. Eurenius, E. Olsson, and M. Kll, Optical antennas based on coupled nanoholes in thin metal films, vol. 3 (2007).

[64] A. Pors, M. G. Nielsen, T. Bernardin, J.-c. Weeber, and S. I. Bozhevolnyi, "Efficient unidirectional polarization-controlled excitation of surface plasmon polaritons," Light: Science \& Applications 3, e197 (2014). 
[65] W. L. Barnes, A. Dereux, and T. W. Ebbesen, "Surface plasmon subwavelength optics," Nature 424, 824-830 (2003).

[66] E. Verhagen, Subwavelength light confinement with surface plasmon polaritons. (Ph.D. thesis, University Utrecht, 2009).

[67] P. B. Johnson and R. W. Christy, "Optical Constants of the Noble Metals," Phys. Rev. B 6, 4370-4379 (1972).

[68] J. Mak, The role of surface plasmons in metal-enhanced chemiluminescence. (Master thesis, University Twente, 2014).

[69] S. W. Prescott and P. Mulvaney, "Gold nanorod extinction spectra," Journal of Applied Physics 99, 123504 (2006).

[70] J. Zuloaga and P. Nordlander, "On the Energy Shift between Near-Field and Far-Field Peak Intensities in Localized Plasmon Systems," Nano Lett. 11, 12801283 (2011).

[71] E. Abbe, "On the estimation of aperture in the microsope," Journal of the Royal Microscopical Society 1, 388-423 (1881).

[72] M. Burresi, Nanoscale investigation of light-matter interactions mediated by magnetic and electric coupling (Ph.D. thesis, University Twente, 2009).

[73] E. H. Synge, "A suggested method for extending microscopic resolution into the ultra-microscopic region," The London, Edinburgh, and Dublin Philosophical Magazine and Journal of Science 6, 356-362 (1928).

[74] D. W. Pohl, W. Denk, and M. Lanz, "Optical stethoscopy: Image recording with resolution /20," Applied Physics Letters 44, 651-653 (1984).

[75] E. Betzig, J. K. Trautman, T. D. Harris, J. S. Weiner, and R. L. Kostelak, "Breaking the Diffraction Barrier: Optical Microscopy on a Nanometric Scale," Science 251, 1468-1470 (1991).

[76] K. Karrai and R. D. Grober, "Piezoelectric tipsample distance control for near field optical microscopes," Applied Physics Letters 66, 1842-1844 (1995).

[77] M. Balistreri, J. Korterik, L. Kiupers, and N. van Hulst, "Phase mapping of optical fields in integrated optical waveguide structures," Journal of Lightwave Technology 19, 1169-1176 (2001).

[78] M. Burresi, R. J. P. Engelen, A. Opheij, D. van Oosten, D. Mori, T. Baba, and L. Kuipers, "Observation of Polarization Singularities at the Nanoscale," Phys. Rev. Lett. 102, 033902 (2009).

[79] A. Zewail, "Laser Femtochemistry," Science 242, 1645-1653 (1988).

[80] E. D. Potter, J. L. Herek, S. Pedersen, Q. Liu, and A. H. Zewail, "Femtosecond laser control of a chemical reaction," Nature 355, 66-68 (1992).

[81] R. S. Judson and H. Rabitz, "Teaching lasers to control molecules," Phys. Rev. Lett. 68, 1500-1503 (1992).

[82] A. Assion, T. Baumert, M. Bergt, T. Brixner, B. Kiefer, V. Seyfried, M. Strehle, and G. Gerber, "Control of Chemical Reactions by Feedback-Optimized PhaseShaped Femtosecond Laser Pulses," Science 282, 919-922 (1998).

[83] J. L. Herek, W. Wohlleben, R. J. Cogdell, D. Zeidler, and M. Motzkus, "Quantum control of energy flow in light harvesting," Nature 417, 533-535 (2002). 
[84] B. Gjonaj, J. Aulbach, P. M. Johnson, A. P. Mosk, L. Kuipers, and A. Lagendijk, Active spatial control of plasmonic fields, vol. 5 (Nature Publishing Group, 2011).

[85] J.-H. Park, C. Park, H. Yu, J. Park, S. Han, J. Shin, S. H. Ko, K. T. Nam, Y.-H. Cho, and Y. Park, "Subwavelength light focusing using random nanoparticles," Nat Photon 7, 454-458 (2013).

[86] E. Prodan, C. Radloff, N. J. Halas, and P. Nordlander, "A Hybridization Model for the Plasmon Response of Complex Nanostructures," Science 302, 419-422 (2003).

[87] T. W. Ebbesen, C. Genet, and S. I. Bozhevolnyi, "Surface-plasmon circuitry," Physics Today 61, 44-50 (2008).

[88] T. Ergin, N. Stenger, P. Brenner, J. B. Pendry, and M. Wegener, "ThreeDimensional Invisibility Cloak at Optical Wavelengths," Science 328, 337-339 (2010).

[89] A. Kinkhabwala, Z. Yu, S. Fan, Y. Avlasevich, K. Mllen, and W. E. Moerner, "Large single-molecule fluorescence enhancements produced by a bowtie nanoantenna," Nature Photonics 3, 654-657 (2009).

[90] P. Zijlstra, P. M. R. Paulo, and M. Orrit, Optical detection of single nonabsorbing molecules using the surface plasmon resonance of a gold nanorod, vol. 7 (Nature Publishing Group, 2012).

[91] J. Dorfmller, D. Dregely, M. Esslinger, W. Khunsin, R. Vogelgesang, K. Kern, and H. Giessen, "Near-Field Dynamics of Optical Yagi-Uda Nanoantennas," Nano Lett. 11, 2819-2824 (2011).

[92] P. Mhlschlegel, H.-J. Eisler, O. J. F. Martin, B. Hecht, and D. W. Pohl, "Resonant Optical Antennas," Science 308, 1607-1609 (2005).

[93] X. Ni, N. K. Emani, a. V. Kildishev, a. Boltasseva, and V. M. Shalaev, "Broadband Light Bending with Plasmonic Nanoantennas," Science 335, 427-427 (2012).

[94] N. Berkovitch, P. Ginzburg, and M. Orenstein, "Concave Plasmonic Particles : Broad-Band Geometrical Tunability in the Near-Infrared," pp. 1405-1408 (2010).

[95] I. D. Mayergoyz, D. R. Fredkin, and Z. Zhang, "Electrostatic (plasmon) resonances in nanoparticles," Physical Review B - Condensed Matter and Materials Physics 72, 155412 (2005).

[96] P. Ginzburg, N. Berkovitch, A. Nevet, I. Shor, and M. Orenstein, "Resonances On-Demand for Plasmonic Nano-Particles," pp. 2329-2333 (2011).

[97] T. Feichtner, O. Selig, M. Kiunke, and B. Hecht, "Evolutionary optimization of optical antennas," Physical Review Letters 109, 1-5 (2012).

[98] E. Prodan and P. Nordlander, "Plasmon hybridization in spherical nanoparticles," The Journal of Chemical Physics 120, 5444-5454 (2004).

[99] F. Wang, Y. R. Shen, M. S. Division, and L. Berkeley, "General Properties of Local Plasmons in Metal Nanostructures," 206806, 1-4 (2006).

[100] G. Lozano, D. J. Louwers, S. R. Rodrguez, S. Murai, O. T. Jansen, M. A. Verschuuren, and J. Gmez Rivas, "Plasmonics for solid-state lighting: enhanced excitation and directional emission of highly efficient light sources," Light Sci Appl 2, e66 (2013). 
[101] J. Aizpurua, P. Hanarp, D. S. Sutherland, M. Kll, G. W. Bryant, and F. J. Garca de Abajo, "Optical Properties of Gold Nanorings," Phys. Rev. Lett. 90, 057401 (2003).

[102] H. B. Jean Lerme, "Size Dependence of the Surface Plasmon Resonance Damping in Metal Nanospheres," Journal of Physical Chemistry Letters 1, 2922 (2010).

[103] J. Kern, S. Gromann, N. V. Tarakina, T. Hckel, M. Emmerling, M. Kamp, J.-S. Huang, P. Biagioni, J. C. Prangsma, and B. Hecht, "Atomic-Scale Confinement of Resonant Optical Fields," Nano Lett. 12, 5504-5509 (2012).

[104] S. A. Maier, "Plasmonics: The benefits of darkness," Nat Mater 8, 699-700 (2009).

[105] Z.-J. Yang, Z.-S. Zhang, L.-H. Zhang, Q.-Q. Li, Z.-H. Hao, and Q.-Q. Wang, "Fano resonances in dipole-quadrupole plasmon coupling nanorod dimers," Opt. Lett. 36, 1542-1544 (2011).

[106] T. G. Habteyes, S. Dhuey, S. Cabrini, P. J. Schuck, and S. R. Leone, "ThetaShaped Plasmonic Nanostructures: Bringing Dark Multipole Plasmon Resonances into Action via Conductive Coupling," Nano Lett. 11, 1819-1825 (2011).

[107] F. Hao, E. M. Larsson, T. A. Ali, D. S. Sutherland, and P. Nordlander, "Shedding light on dark plasmons in gold nanorings," Chemical Physics Letters 458, 262-266 (2008).

[108] M. Liu, T.-W. Lee, S. K. Gray, P. Guyot-Sionnest, and M. Pelton, "Excitation of Dark Plasmons in Metal Nanoparticles by a Localized Emitter," Phys. Rev. Lett. 102, 107401 (2009).

[109] N. Verellen, Y. Sonnefraud, H. Sobhani, F. Hao, V. V. Moshchalkov, P. V. Dorpe, P. Nordlander, and S. A. Maier, "Fano Resonances in Individual Coherent Plasmonic Nanocavities 2009," (2009).

[110] F. Shafiei, F. Monticone, K. Q. Le, X.-X. Liu, T. Hartsfield, A. Al, and X. Li, "A subwavelength plasmonic metamolecule exhibiting magnetic-based optical Fano resonance," Nat Nano 8, 218-218 (2013).

[111] M. Hentschel, M. Saliba, R. Vogelgesang, H. Giessen, A. P. Alivisatos, and N. Liu, "Transition from Isolated to Collective Modes in Plasmonic Oligomers," Nano Lett. 10, 2721-2726 (2010).

[112] D. Vercruysse, Y. Sonnefraud, N. Verellen, F. B. Fuchs, G. Di Martino, L. Lagae, V. V. Moshchalkov, S. A. Maier, and P. Van Dorpe, "Unidirectional side scattering of light by a single-element nanoantenna," Nano Letters 13, 3843-3849 (2013).

[113] S. Mukherjee, H. Sobhani, J. B. Lassiter, R. Bardhan, P. Nordlander, and N. J. Halas, "Fanoshells: Nanoparticles with Built-in Fano Resonances," Nano Lett. 10, 2694-2701 (2010).

[114] C. M. Dutta, T. A. Ali, D. W. Brandl, T.-H. Park, and P. Nordlander, "Plasmonic properties of a metallic torus," The Journal of Chemical Physics 129, 084706 (2008).

[115] Y. Sonnefraud, N. Verellen, H. Sobhani, G. A. Vandenbosch, V. V. Moshchalkov, P. Van Dorpe, P. Nordlander, and S. A. Maier, "Experimental Realization of Subradiant, Superradiant, and Fano Resonances in Ring/Disk Plasmonic Nanocavities," ACS Nano 4, 1664-1670 (2010). 
[116] H. Aouani, J. Wenger, D. Grard, H. Rigneault, E. Devaux, T. W. Ebbesen, F. Mahdavi, T. Xu, and S. Blair, "Crucial Role of the Adhesion Layer on the Plasmonic Fluorescence Enhancement," ACS Nano 3, 2043-2048 (2009).

[117] T. Schumacher, K. Kratzer, D. Molnar, M. Hentschel, H. Giessen, and M. Lippitz, "Nanoantenna-enhanced ultrafast nonlinear spectroscopy of a single gold nanoparticle," Nat Commun 2, 333 (2011).

[118] P. Offermans, M. C. Schaafsma, S. R. K. Rodriguez, Y. Zhang, M. CregoCalama, S. H. Brongersma, and J. Gmez Rivas, "Universal Scaling of the Figure of Merit of Plasmonic Sensors," ACS Nano 5, 5151-5157 (2011).

[119] S. R. K. Rodriguez, S. Murai, M. A. Verschuuren, and J. G. Rivas, "LightEmitting Waveguide-Plasmon Polaritons," Phys. Rev. Lett. 109, 166803 (2012).

[120] A. Lovera, B. Gallinet, P. Nordlander, and O. J. Martin, "Mechanisms of Fano Resonances in Coupled Plasmonic Systems," ACS Nano 7, 4527-4536 (2013).

[121] a. Degiron, H. J. Lezec, N. Yamamoto, and T. W. Ebbesen, "Optical transmission properties of a single subwavelength aperture in a real metal," Optics Communications 239, 61-66 (2004).

[122] H. F. Ghaemi and T. Thio, "Extraordinary optical transmission through subwavelength hole arrays," Nature 391, 667-669 (1998).

[123] N. García and M. Bai, "Theory of transmission of light by sub-wavelength cylindrical holes in metallic films." Optics express 14, 10028-10042 (2006).

[124] L. Yin, V. K. Vlasko-vlasov, A. Rydh, J. Pearson, and U. Welp, "Surface plasmons at single nanoholes in Au films," Applied Physics Letters 85, 467469 (2004).

[125] J. Alegret, P. Johansson, and M. Käll, "Green's tensor calculations of plasmon resonances of single holes and hole pairs in thin gold films," New Journal of Physics 10, 105004 (2008).

[126] A. Degiron, E. Laux, E. Devaux, C. Genet, J. Alegret, J. Yi, A. Cuche, and F. D. Leo, "Diffraction Regimes of Single Holes," 023901, 1-5 (2012).

[127] C. Genet and T. W. Ebbesen, "Light in tiny holes." Nature 445, 39-46 (2007).

[128] H. A. Bethe, "Theory of Diffraction by Small Holes," Phys. Rev. 66, 163-182 (1944).

[129] B. l. Feber, N. Rotenberg, D. M. Beggs, and L. Kuipers, "Simultaneous measurement of nanoscale electric and magnetic optical fields," Nat Photon 8, 43-46 (2014).

[130] C. Snnichsen, A. C. Duch, G. Steininger, M. Koch, G. v. Plessen, and J. Feldmann, "Launching surface plasmons into nanoholes in metal films," Applied Physics Letters 76, 140-142 (2000).

[131] L. Yin, V. K. Vlasko-vlasov, A. Rydh, J. Pearson, and U. Welp, "Surface plasmons at single nanoholes in Au films," Applied Physics Letters 85, 467469 (2004).

[132] S.-H. Chang, S. Gray, and G. Schatz, "Surface plasmon generation and light transmission by isolated nanoholes and arrays of nanoholes in thin metal films," Opt. Express 13, 3150-3165 (2005). 
[133] A.-L. Baudrion, F. de Leon-Perez, O. Mahboub, A. Hohenau, H. Ditlbacher, F. J. Garcia-Vidal, J. Dintinger, T. W. Ebbesen, L. Martin-Moreno, and J. R. Krenn, "Coupling efficiency of light to surface plasmon polariton for single subwavelength holes in a gold film," Opt. Express 16, 3420-3429 (2008).

[134] F. J. Garcia-Vidal, L. Martin-Moreno, T. W. Ebbesen, and L. Kuipers, "Light passing through subwavelength apertures," Rev. Mod. Phys. 82, 729-787 (2010).

[135] D. Griffiths, Introduction to electrodynamics. (Prentice Hall, 1999).

[136] R. Gordon and A. Brolo, "Increased cut-off wavelength for a subwavelength hole in a real metal." Optics express 13, 1933-1938 (2005).

[137] F. J. Garca-Vidal, E. Moreno, J. A. Porto, and L. Martn-Moreno, "Transmission of Light through a Single Rectangular Hole," Phys. Rev. Lett. 95, 103901 (2005).

[138] F. J. Garca-Vidal, L. Martn-Moreno, E. Moreno, L. K. S. Kumar, and R. Gordon, "Transmission of light through a single rectangular hole in a real metal," Phys. Rev. B 74, 153411 (2006).

[139] "CST microwave studio," .

[140] E. Betzig and R. J. Chichester, "Single Molecules Observed by Near-Field Scanning Optical Microscopy," Science 262, 1422-1425 (1993).

[141] J. A. Veerman, M. F. Garcia-Parajo, L. Kuipers, and N. F. van Hulst, "Single molecule mapping of the optical field distribution of probes for near-field microscopy," J Microsc 194, 477-482 (1999).

[142] N. F. v. Hulst, J.-A. Veerman, M. F. Garca-Paraj, and L. K. Kuipers, "Analysis of individual (macro)molecules and proteins using near-field optics," The Journal of Chemical Physics 112, 7799-7810 (2000).

[143] B. Sick, B. Hecht, U. P. Wild, and L. Novotny, "Probing confined fields with single molecules and vice versa," Journal of Microscopy 202, 365-373 (2001).

[144] R. Moerland, N. van Hulst, H. Gersen, and L. Kuipers, "Probing the negative permittivity perfect lens at optical frequencies using near-field optics and single molecule detection," Opt. Express 13, 1604-1614 (2005).

[145] H. Shin, P. B. Catrysse, and S. Fan, "Effect of the plasmonic dispersion relation on the transmission properties of subwavelength cylindrical holes," Phys. Rev. B 72, 085436 (2005).

[146] A. Naber, D. Molenda, U. C. Fischer, H.-J. Maas, C. Hppener, N. Lu, and H. Fuchs, "Enhanced Light Confinement in a Near-Field Optical Probe with a Triangular Aperture," Phys. Rev. Lett. 89, 210801 (2002).

[147] A. Degiron, H. J. Lezec, N. Yamamoto, and T. W. Ebbesen, "Optical transmission properties of a single subwavelength aperture in a real metal," Optics Communications 239, 61-66 (2004).

[148] J. Wang, W. Zhou, and E.-P. Li, "Enhancing the light transmission of plasmonic metamaterials through polygonal aperture arrays," Optics Express 17, 20349 (2009).

[149] F. Ye, J. M. Merlo, M. J. Burns, and M. J. Naughton, "Optical and electrical mappings of surface plasmon cavity modes," Nanophotonics 3, 33-49 (2014). 
[150] A. Salomon, M. Zielinski, R. Kolkowski, J. Zyss, and Y. Prior, "Size and Shape Resonances in Second Harmonic Generation from Silver Nanocavities," J. Phys. Chem. C 117, 22377-22382 (2013).

[151] B. l. Feber, Nanoscale electric and magnetic optical vector fields (Ph.D. thesis, University Twente, 2015).

[152] R. J. P. Engelen, Y. Sugimoto, H. Gersen, N. Ikeda, K. Asakawa, and L. K. Kuipers, "Ultrafast evolution of photonic eigenstates in k-space," Nat Phys 3, 401-405 (2007).

[153] B. Gjonaj, J. Aulbach, P. M. Johnson, A. P. Mosk, L. Kuipers, and A. Lagendijk, "Optical control of plasmonic bloch modes on periodic nanostructures," Nano Letters 12, 546-550 (2012).

[154] B. Gjonaj, J. Aulbach, P. M. Johnson, A. P. Mosk, L. Kuipers, and A. Lagendijk, "Focusing and scanning microscopy with propagating surface plasmons," Physical Review Letters 110, 28-32 (2013).

[155] Y. Alaverdyan, B. Seplveda, L. Eurenius, E. Olsson, and M. Kll, Optical antennas based on coupled nanoholes in thin metal films, vol. 3 (2007).

[156] E. Hecht, Optics (Pearson Education Limited, 2014), 4th ed.

[157] M. Born and E. Wolf, Principle of Optics (Cambridge, university press, 1999), 7 th ed.

[158] M. Burresi, R. J. P. Engelen, A. Opheij, D. van Oosten, D. Mori, T. Baba, and L. Kuipers, "Observation of Polarization Singularities at the Nanoscale," Phys. Rev. Lett. 102, 033902 (2009).

[159] A. d. Hoogh, N. Rotenberg, and L. Kuipers, "Optical singularities in plasmonic fields near single subwavelength holes," J. Opt. 16, 114004 (2014).

[160] B. Gjonaj, J. Aulbach, P. M. Johnson, A. P. Mosk, L. Kuipers, and A. Lagendijk, "Active spatial control of plasmonic fields," Nat Photon 5, 360-363 (2011).

[161] C. A. Balanis, Modern antenna handbook (John Wiley \& Sons, 2008).

[162] T. Coenen and A. Polman, "Optical Properties of Single Plasmonic Holes Probed with Local Electron Beam Excitation," ACS Nano 8, 7350-7358 (2014).

[163] M. R. Dennis, N. I. Zheludev, and F. J. Garca de Abajo, "The plasmon Talbot effect," Optics Express 15, 9692 (2007).

[164] W. Zhao, X. Huang, and Z. Lu, "Super Talbot effect in indefinite metamaterial," Optics Express 19, 15297 (2011).

[165] D. van Oosten, M. Spasenovi, and L. Kuipers, "Nanohole Chains for Directional and Localized Surface Plasmon Excitation," Nano Lett. 10, 286-290 (2010).

[166] A. Gangnaik, Y. M. Georgiev, B. McCarthy, N. Petkov, V. Djara, and J. D. Holmes, "Characterisation of a novel electron beam lithography resist, SML and its comparison to PMMA and ZEP resists," Microelectronic Engineering 123, 126-130 (2014).

[167] A. N. Broers, A. C. F. Hoole, and J. M. Ryan, "Electron beam lithographyResolution limits," Microelectronic Engineering 32, 131-142 (1996).

[168] J.-S. Huang, V. Callegari, P. Geisler, C. Brning, J. Kern, J. C. Prangsma, X. Wu, T. Feichtner, J. Ziegler, P. Weinmann, M. Kamp, A. Forchel, P. Biagioni, U. Sennhauser, and B. Hecht, "Atomically flat single-crystalline gold nanostructures for plasmonic nanocircuitry," Nat Commun 1, 150 (2010). 
[169] M. Kuttge, E. J. R. Vesseur, J. Verhoeven, H. J. Lezec, H. A. Atwater, and A. Polman, "Loss mechanisms of surface plasmon polaritons on gold probed by cathodoluminescence imaging spectroscopy." Applied Physics Letters 93, 113110 (2008).

[170] C. Rewitz, G. Razinskas, P. Geisler, E. Krauss, S. Goetz, M. Pawowska, B. Hecht, and T. Brixner, "Coherent Control of Plasmon Propagation in a Nanocircuit," Phys. Rev. Applied 1, 014007 (2014).

[171] T. L. Chen, D. J. Dikken, J. C. Prangsma, F. Segerink, and J. L. Herek, "Characterization of Sierpinski carpet optical antenna at visible and near-infrared wavelengths," New J. Phys. 16, 093024 (2014).

[172] C. Kan, X. Zhu, and G. Wang, "Single-Crystalline Gold Microplates: Synthesis, Characterization, and Thermal Stability," J. Phys. Chem. B 110, 4651-4656 (2006).

[173] Z. Guo, Y. Zhang, Y. DuanMu, L. Xu, S. Xie, and N. Gu, "Facile synthesis of micrometer-sized gold nanoplates through an aniline-assisted route in ethylene glycol solution," Colloids and Surfaces A: Physicochemical and Engineering Aspects 278, 33-38 (2006).

[174] X. Sun and Y. Luo, "On the preparation of single-crystalline gold microplates," Materials Letters 60, 3145-3148 (2006).

[175] N. Verellen, Y. Sonnefraud, H. Sobhani, F. Hao, V. V. Moshchalkov, P. V. Dorpe, P. Nordlander, and S. A. Maier, "Fano Resonances in Individual Coherent Plasmonic Nanocavities," Nano Lett. 9, 1663-1667 (2009).

[176] R. L. Olmon, M. Rang, P. M. Krenz, B. A. Lail, L. V. Saraf, G. D. Boreman, and M. B. Raschke, "Determination of Electric-Field, Magnetic-Field, and Electric-Current Distributions of Infrared Optical Antennas: A Near-Field Optical Vector Network Analyzer," Phys. Rev. Lett. 105, 167403 (2010).

[177] M. Schnell, A. Garca-Etxarri, A. J. Huber, K. Crozier, J. Aizpurua, and R. Hillenbrand, "Controlling the near-field oscillations of loaded plasmonic nanoantennas," Nat Photon 3, 287-291 (2009).

[178] M. Esslinger and R. Vogelgesang, "Reciprocity Theory of Apertureless Scanning Near-Field Optical Microscopy with Point-Dipole Probes," ACS Nano 6, 81738182 (2012).

[179] J. A. Porto, R. Carminati, and J.-J. Greffet, "Theory of electromagnetic field imaging and spectroscopy in scanning near-field optical microscopy," Journal of Applied Physics 88, 4845-4850 (2000).

[180] "http://www.thehistoryblog.com/archives/26724," . 


\section{Samenvatting voor allen}

Het manipuleren van elektromagnetische golven is iets waar de mensheid zich al eeuwen mee bezig houdt. Interessant is dat één van de eerste voorbeelden hiervan te maken heeft met het manipuleren van elektromagnetische golven in het zichtbare spectrum, door gebruik te maken van optische antennes. Een vaak aangehaald voorbeeld is de Lycurgus kelk die rond de tweede eeuw na Christus gemaakt is door de Romeinen. Wanneer deze kelk van achter wordt beschenen met een witte licht bron, laat de kelk een palet van kleuren zien variërend van groen tot intens rood, zie Figuur 1. De makers van deze kelk hebben het voor elkaar gekregen om een materiaal te creëren waarbij de absorptie en de verstrooiing van licht bij verschillende kleuren plaats vindt. Uit analyse blijkt dat in het glasachtige materiaal kleine 50 nanometer gouddeeltjes

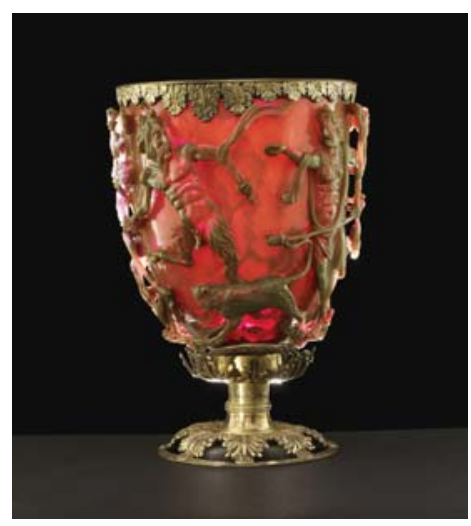

Figuur 1: Lycurgus kelk. Oude romeinse kelk die door de aanwezigheid van kleine metalische deeltjes in het glas een groene dan wel rode verschijning heeft, wanneer licht wordt gereflecteerd dan wel geabsorbeerd. Plaatje overgenomen van [180]. 
zijn versmolten. In de introductie van dit proefschrift is beschreven dat deze kleine metaaldeeltjes inderdaad de eigenschap hebben om bij specifieke kleuren sterk het licht te verstrooien.

In de twintigste eeuw is de fascinatie voor materialen, gemaakt met deze kleine metaaldeeltjes, nog onverminderd groot, wat voort komt uit het feit dat deze deeltjes licht kunnen manipuleren en lokaliseren op de nanometerschaal. Het manipuleren van elektromagnetische golven op een sub-golflengteschaal is niet een nieuw concept op zichzelf. Al jaren worden antennes in de telecommunicatie gebruikt om elektromagnetische signalen op te vangen en te geleiden naar een elektronisch circuit en visa versa. Conceptueel zijn deze nanodeeltjes vrijwel identiek aan de antennes die we kennen en deze worden daarom ook vaak optische antennes genoemd. Een kenmerkende eigenschap van antennes is, dat er een relatie is tussen hun fysieke dimensies en de golflengte waarbij deze resoneert. Deze relatie is gedetailleerd beschreven in hoofdstuk 1 . Om een antenne te maken in het optische regiem zal deze een dimensie moeten hebben in de orde van \pm .0000006 meter. Recente technologische vooruitgangen in de fabricatie van nanostructuren met dimensies van enkele tientallen nanometers, hebben in de afgelopen tien jaar tot de bloei van een nieuw wetenschappelijk veld geleid: het veld van Plasmonics. Er zijn reeds verschillende soorten optische antennes ontwikkeld die hebben geleid tot een verhoogde efficiëntie van verschillende optische toepassingen zoals: LED verlichting, zonnepanelen en de ontwikkeling van gevoelige meetmethode voor de detectie van lage concentraties organische en inorganische stoffen. Een voorbeeld waarbij optische antennes van meerwaarde zijn gebleken, is, het gebruik van een laag van optische antennes als een anti-reflectie laag op zonnecellen. De optische antennes weten het licht efficiënt in het materiaal te koppelen en vast te houden, resulterend in een hogere efficiëntie van de zonnecel. De vrijheid waarmee licht op de nanoschaal kan worden gemanipuleerd, gaat gepaard met een gevoeligheid voor de vorm en de materiaalkeuze van de antenne en zijn omgeving. Tot op heden is er nog geen alomvattende theorie voor het ontwerpen van optische antennes, waardoor er na tien jaar nog altijd levendig studie wordt verricht naar de optische effecten van verschillende antenne vormen.

Dit proefschrift presenteert een gedetailleerde studie waarin verschillende optische eigenschappen, waaronder kleur, gevoeligheid, en stralingspatroon van een antenne, worden gemanipuleerd door de aspecten vorm, koppeling en belichting te varieëren. Deze aspecten worden respectievelijk behandeld in de hoofdstukken 3, 4 en 5-6. 
Hoofdstuk 3 gaat in op de spectrale afhankelijkheid van de vorm van een optische antenne. Door de antenne een wiskundige vorm te geven, waarbij de breedte van de antenne wordt gemoduleerd met een sinusfunctie, is een geleidelijke transitie tussen een holle en bolle antennevorm gerealiseerd. Door deze geleidelijke transitie is het mogelijk om de relatie tussen deze twee verschillende vormen en hun effect op de kleur, waarbij de optische antennes resoneren, te beschrijven. De kleur waarbij deze vernieuwende antennes resoneren, blijkt bij een relatief kleine verandering van antennevorm extreem te kunnen worden verschoven. Deze eigenschap, in combinatie met zijn kleine vorm, is zeer interessant voor veel optische toepassingen.

Door het dicht bij elkaar brengen van twee optische antennes ontstaan nieuwe, gekoppelde resonanties. In hoofdstuk 4 wordt getoond dat de gevoeligheid van de koppelingsresonanties voor licht, sterk afhangt van de symmetrie van de koppeling en de gevoeligheid van de fundamentele antennes. Hierbij zijn we vooral geïntereseerd in het koppelen van antennes die in meer of mindere mate gevoelig zijn voor licht. Met een gekoppeld, harmonisch oscillatormodel wordt duidelijk dat de gevoeligheid van een ongevoelige antenne kan worden beïnvloed door deze te koppelen met een andere antenne. De mate van versterking die kan worden behaald hangt af van de gevoeligheidsratio van de twee optische antennes.

In hoofdstuk $\mathbf{5}$ en $\mathbf{6}$ staat de transmissie van licht door sub-golflengte gat-antennes centraal met het doel om hun nabije velddistributie te sturen. Hiervoor worden verschillende antennereeksen geïntroduceerd, bestaande uit 2 tot 10 gat-antennes, die met een nieuwe techniek individueel belicht worden. De reeksen van gat-antennes worden belicht met diverse fase-patronen, waardoor de richting van de gegenereerde oppervlaktegolven wordt gestuurd. Tenslotte laten we zien dat met deze vernieuwende techniek, naast het realiseren van unieke nog niet eerder gecreërde optische oppervlaktegolven, ook de polarisatietoestand van deze oppervlaktegolven te programmeren zijn. 


\section{Dankwoord}

Nu kijken we nog in een wazige spiegel, maar straks staan we oog in oog. $\mathrm{Nu}$ is mijn kennen nog beperkt, maar straks zal ik volledig kennen, zoals ik zelf gekend ben.

1 Korintiërs 13:12

Tijdens de vijf jaar van mijn onderzoek bij de Optical Sciences Group heb ik mogen samenwerken met een verscheidenheid van gemotiveerde en getalenteerde mensen die stuk voor stuk een bijdrage hebben geleverd aan de inhoud van dit proefschrift.

Als eerste wil ik mijn begeleider bedanken. Jennifer, ik wil je bedanken voor je vertrouwen in mij, de ondersteuning door de jaren heen en voor de vrijheid die je me gaf in het opzetten van een nieuwe onderzoekstak in de groep. De open en familiere sfeer in de groep en de laagdrempeligheid waarmee iedereen te benaderen is, zijn een reflectie van jouw karakter.

Jord, met jouw komst in de groep, eerst één dag in de week en later full time, ben je van grote invloed geweest op mijn ontwikkelling als onderzoeker en ben je betrokken geweest bij alle resultaten die in dit proefschrift staan. Ik heb genoten van de uren die we hebben gediscussiëerd over experimentele resultaten en het samen brainstormen over de volgende stappen in ons onderzoek. Jouw liefde voor de fysica is erg aanstekelijk en ik heb de indruk dat ik je zelfs 's avonds wakker kan maken om nieuwe resultaten te bespreken (al heb ik dat nog niet geprobeerd). Voor jouw interesse en toewijding voor/aan mijn onderzoek ben ik je zeer dankbaar.

Frans en Jeroen, beide hebben jullie een grote bijdrage geleverd aan de technische aspecten die nodig waren voor respectievelijk het maken van de nano-structuren en het ontwikkelen van de electronica van de meetopstellingen. Ik ben er van overtuigd dat veel van de resultaten die in 
dit proefschrift staan, zonder jullie bijdrage er niet zo fraai zouden hebben uitgezien. Frans, de lange dagen in de cleanroom, waarbij we toch vaak aan het wachten waren tot de BAK 600 eindelijk de noodzakelijke $10^{-6}$ mbar haalde, waren niet saai. Ik kijk met plezier terug op onze gesprekken en op jouw nuchtere kijk op het leven. Jeroen ik heb genoten van de tijd waarin we samen hebben gewerkt aan het vernieuwen van de NSOM. Er zijn vaak duizend en één manieren om een probleem te tackelen, maar jij weet het toch vaak het orgineelst en het elegantst te doen. Je blijft voor mij toch echt de 'electronicatovenaar'.

Simon, tijdens het verhuizen van de NSOM van COPS terug naar OS en de tijd die we samen hebben doorgebracht bij de wederopbouw, waardeerde ik je pragmatische blik en je dagelijkse doelgerichtheid.

Alexander, Jorick, Andrew, Erik, Amandev, Dan, Bart en Stefan, jullie brachten ontspanning, gezelligheid en humor in en rond het lab. De ontspannen momenten in de koffiepauzes, onze ping pong tournooien en de 'beer o'clock', zullen me altijd bijblijven. Mijn dank gaat ook uit naar de andere leden van Optical Sciences group, in het bijzonder: Herman, Sonia, Annemarie, Karen, Meindert, Rick, Pablo, Qing, Mustafa, Gerwin, Sergio, Yean-Sheng, David v. D. en David T., Bente, Lourens, Joris en Cederic.

Ik wil mijn familie bedanken voor hun blijvende interesse en aanmoediging. In het bijzonder wil ik mijn moeder bedanken voor de jaren van toewijding en de offers die gemaakt zijn zodat ik mij kon focussen op mijn studie. Charlotte, bedankt voor het redigeren van mijn thesis ... de lezers zullen je dankbaar zijn. Elisabeth, bedankt voor de rustige momenten die jij in het afgelopen jaar creëerde, ... door extra op Jonathan te passen. Matthijs, bedankt voor de gezelligheid en onze fietstochten in de zomeravonden. Charlotte en Simon, bedankt dat jullie mij als paranimf willen bijstaan tijdens mijn verdediging.

Jonathan, ik wil jou bedanken voor alle vijf-minuten-pauzes tijdens de lange marathon van het schrijven van dit proefschrift. Deze korte momentjes met jou gaven mij de kracht om door te gaan.

Jackeline, hoe kan ik in een paar regels recht doen aan jouw dagelijkse bijdrage? In de periode van mijn onderzoek bouwde je me op wanneer ik het even niet zag zitten. Op vele manieren heb jij ons gezinnetje gedragen tijdens de drukke periode van het schrijven. Jij bent de constante in mijn leven. Ik zie uit naar onze volgende avonturen. 